\title{
ESTUdios TEÓRICOS Y OBSERVACIONALES DE ESTRELLAS DE NEUTRONES EN DIFERENTES ESCENARIOS ASTROFÍSICOS
}

\author{
Lic. Federico García
}

\author{
Director: Dr. Jorge A. Combi \\ Co-Directora: Dra. Deborah N. Aguilera
}
Tesis para optar por el grado de
DoCTOR EN ASTRONOMÍA

La Plata, 30 de marzo de 2016

Facultad de Ciencias Astronómicas y Geofísicas Universidad Nacional de La Plata
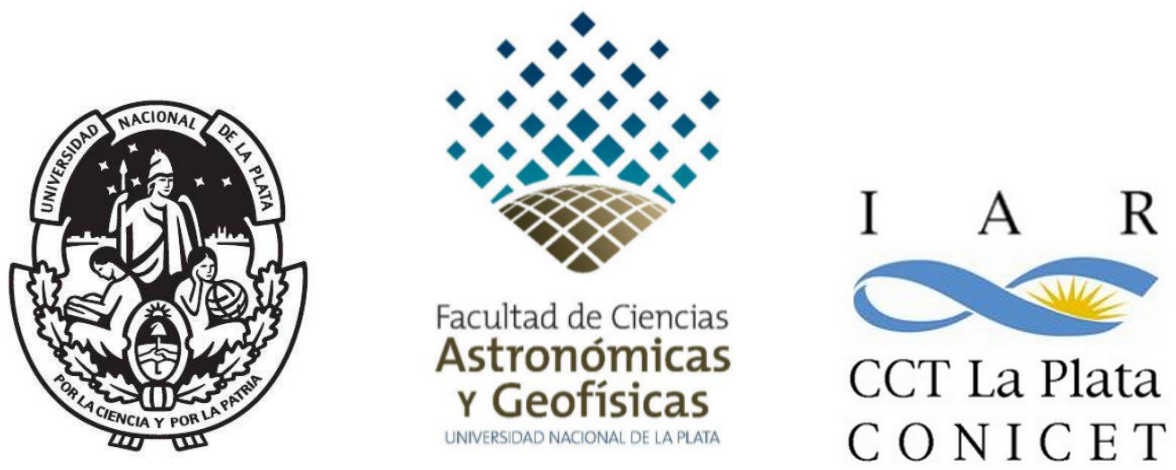

A Julia por volver, a mis viejos por ser, a mis amigos por estar. 



\section{Agradecimientos}

A mis viejos que son y están apoyando todo proyecto y especialmente a papá que trajo las estrellas hasta casa con pasión y profundo conocimiento del cielo. A mis hermanas Loli y Maru, compañeras por siempre. A la abuela y la vida.

A mis amigos de siempre: Ale, Fede, Fran, Maxi, Nacho, Octi, Tuna, al Cocoon y lxs IntisPucas. A Mara y todo Derecho al Cielo Nocturno, un oasis para nuestra Astronomía. A la música que me acompañó en esta etapa. A Astromonos por componerla y ejecutarla, y a Lucas, por ayudarme a descubrirla.

En el plano científico, agradezco fundamentalmente a la Facultad de Ciencias Astronómicas y Geofísicas de la Universidad de La Plata, el Obser, y al Instituto Argentino de Radioastronomía, el IAR, donde siempre encontré espacio para trabajar, pensar y disfrutar (y, por qué no, también sufrir de a ratos) esta etapa de la vida académica. A Gustavo y al Grupo GARRA por abrir horizontes y oportunidades para discutir la ciencia, la técnica e incluso la filosofía. A CONICET y al pueblo argentino por financiarnos.

A todos mis colaboradores de quienes aprendí tanto. Por las aventuras en Groningen: gracias Mariano por todo el impulso y apoyo incondicional, y gracias Guobao por tu ayuda y humildad, y por extensión a todo el Kapteyn Astronomical Institute. De este lado del océano, a Florencia, y en especial a Ignacio, enorme compañero.

Agradezco principalmente a mis directores Jorge y Deborah por las ideas, discusiones, trabajo y libertad que hicieron posible esta tesis, y a mis jurados Héctor Vucetich, Leonardo Pellizza y Félix Mirabel por su lectura crítica y aportes finales.

Finalmente, a Julia por enseñarme el amor y embellecer la vida. 



\section{Resumen}

El objetivo general de este trabajo es realizar aportes al estudio de estrellas de neutrones con campos magnéticos extremos, incluyendo estrellas de neutrones aisladas, inmersas en remanentes de supernova y pertenecientes a sistemas binarios. Se consideraron similitudes y diferencias en las manifestaciones observacionales de estos objetos, producto de la configuración de su campo magnético y su evolución, así como también el efecto que generan en el medio interestelar circundante. Esta investigación abarca desde estrellas de neutrones con campos magnéticos intensos, o magnetares $\left(B>10^{14} \mathrm{G}\right)$ como los pulsares anómalos de rayos $\mathrm{X}$ y los repetidores de rayos $\gamma$, hasta sus contrapartes de campo magnético muy bajo, $B<10^{10} \mathrm{G}$ (también llamadas anti-magnetares), tales como los objetos compactos centrales descubiertos en el interior de remanentes de supernova, y estrellas de neutrones que acretan materia proveniente de una estrella compañera donante en sistemas binarios.

En primer lugar, en esta tesis se introduce un marco actualizado en lo que refiere al conocimiento teórico relacionado con las estrellas de neutrones y sus entornos seguido por una descripción de los satélites de rayos X y las técnicas observacionales empleadas. La parte central involucra los aportes originales realizados que incluyen, primero, los resultados obtenidos producto del desarrollo de modelos teóricos que buscan dar explicación a observaciones realizadas en escenarios astrofísicos asociados a estrellas de neutrones, y en segundo lugar, los resultados correspondientes a estudios observacionales llevados a cabo fundamentalmente en la banda de los rayos X, pero que incorporan datos obtenidos en diferentes longitudes de onda, desde radio hasta rayos-gamma. De esta manera, en esta tesis se ha hecho uso de los más avanzados instrumentos disponibles en la actualidad, con el objetivo de relacionar observación y teoría para lograr un avance sustentable en temas de astrofísica relativista.

Se incorpora además a esta tesis el modelado de estrellas de neutrones en teorías alternativas a la relatividad general, el cual ha despertado un fuerte interés en la comunidad por dos motivos. Por un lado, ya que se piensa que en estas teorías es posible alojar estrellas de neutrones más masivas que en relatividad general, ampliando el límite de $\sim 2 \mathrm{M}_{\odot}$, descripto por las ecuaciones de estado de materia realistas que se proponen actualmente. Recíprocamente, el estudio de la estructura de estrellas de neutrones podría resultar eficaz para poner cotas a los parámetros libres de estas teorías, o incluso encontrar anomalías impuestas por los campos de materia representados a través diferentes ecuaciones de estado en el régimen de campo gravitacional fuerte.

En la Parte I de esta tesis se expone una breve introducción al estado del arte del conocimiento 
de las estrellas de neutrones, desde un punto de vista teórico, abarcando los escenarios astrofísicos que involucran sus diversas manifestaciones observacionales, y se describen las características de los instrumentos disponibles en la banda de los rayos X, así como los lineamientos de las técnicas utilizadas en los estudios observacionales desarrollados.

En la Parte II se presentan dos modelos teóricos que podrían explicar satisfactoriamente observaciones de estrellas de neutrones en las que el campo magnético juega un papel crucial. En el Capítulo 2 se estudia la evolución del campo magnético en una estrella de neutrones que acreta materia proveniente del viento de una estrella compañera de gran masa, con el objeto de analizar la posibilidad de la formación de jets en sistemas binarios con fulguraciones transitorias gigantes de rayos X (García et al., 2014). En el Capítulo 3 mostramos cómo la evolución de un campo magnético toroidal intenso podría ser responsable del frenado repentino conocido como "anti-glitch" en un magnetar (García \& Ranea-Sandoval, 2015).

La Parte III abarca resultados propios obtenidos a partir de estudios observacionales llevados a cabo en la banda de los rayos X. El Capítulo 4 está dedicado a la búsqueda de un remanente compacto central en un remanente de supernova, y el análisis de la emisión extendida asociada (García et al., 2012a,b), realizado a partir de observaciones de archivo de XMM-Newton y Chan$d r a$, y el Capítulo 5 comprende el estudio del enfriamiento de una estrella de neutrones posterior a las erupciones de rayos $\mathrm{X}$ de Tipo-I, detectadas con el satélite $R X T E$, y sus consecuencias sobre la determinación precisa de la masa y el radio de la estrella (García et al., 2013).

La Parte IV engloba los aportes realizados al estudio de estrellas de neutrones en teorías de gravedad alternativas a la relatividad general. En el Capítulo 6 se presentan los resultados obtenidos para el cálculo perturbativo de configuraciones de estrellas de neutrones estáticas y esféricamente simétricas utilizando ecuaciones de estado realistas en el marco de una teoría $f(R)$ métrica (Orellana et al., 2013). El Capítulo 7 contiene la extensión de este estudio a una teoría $f(R)$ bajo el formalismo de Palatini, donde las ecuaciones son resueltas de manera exacta. En ambas teorías se encuentra que los perfiles internos que representan la estructura de las estrellas muestran un comportamiento anti-intuitivo que depende fuertemente de la ecuación de estado utilizada (Teppa Pannia et al., 2016).

Finalmente en la Parte V se exponen las conclusiones generales y el trabajo a futuro que dará continuidad y permitirá profundizar las líneas de investigación iniciadas en esta tesis.

Este trabajo fue realizado paralelamente en el Instituto Argentino de Radioastronomía (IAR) y en la Facultad de Ciencias Astronómicas y Geofísicas de la Universidad Nacional de La Plata (FCAGLP) entre abril del año 2011 y diciembre de 2015. Tanto en IAR, como en FCAGLP, trabajé junto a mi director el Dr. Jorge A. Combi y varios colaboradores. Considerables aportes fueron además obtenidos en viajes realizados al Laboratorio Tandar, donde se desempeña mi co-directora, la Dra. Deborah N. Aguilera, y durante dos estadías de trabajo en el Kapteyn Astronomical Institute de la Universidad de Groningen, en Holanda, donde colaboré con el Prof. Dr. Mariano Méndez. Durante todo el período en que desarrollé esta tesis, conté con becas doctorales del Consejo Nacional de Investigaciones Científicas y Técnicas: los primeros 3 años, la Beca de Postgrado Tipo I y en los restantes la de Finalización de Doctorado, o Tipo II. 


\section{Índice general}

I Introducción 1

1. Estrellas de neutrones 3

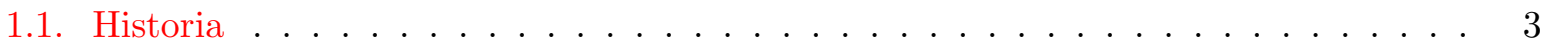

1.2. Características generales de las estrellas de neutrones . . . . . . . . . . . . 6

1.2.1. Propiedades físicas macroscópicas . . . . . . . . . . . . . . 7

1.2.2. El diagrama $P-\dot{P} \ldots \ldots \ldots \ldots \ldots \ldots \ldots$

1.2.3. Campo magnético y clasificación, desde magnetares hasta CCOs . . . . . 9

1.2.4. Estructura interna . . . . . . . . . . . . . . . . . . . 10

1.2.5. Anomalías en la rotación: glitches y anti-glitches . . . . . . . . . . . . . 12

1.2.6. Estrellas de neutrones en sistemas binarios . . . . . . . . . . . . . . . 13

1.3. Modelos y configuraciones de estrellas de neutrones . . . . . . . . . . . . . . . 19

1.3.1. Estrellas de neutrones en relatividad general . . . . . . . . . . . . . . 19

1.3.2. La ecuación de estado . . . . . . . . . . . . . . . . . . . . 22

1.3.3. El diagrama Masa-Radio . . . . . . . . . . . . . . . . . . . 24

1.3.4. Configuraciones axisimétricas de estrellas de neutrones . . . . . . . . . . 26

1.3.5. Estrellas de neutrones en teorías alternativas de gravedad . . . . . . . . . . . 28

1.4. Modelos de evolución del campo magnético . . . . . . . . . . . . . . . . . . . . . . . . . . 31

1.4.1. Propiedades de transporte de la corteza . . . . . . . . . . . . . . . . 31

1.4.2. Evolución del campo magnético . . . . . . . . . . . . . . . . . . . . . . . . . . . 33

1.5. Las estrellas de neutrones desde los rayos $\mathrm{X} \ldots \ldots \ldots \ldots$. . . . . . . . . . . . . . . . . . . . . . . . .

1.5.1. Observatorios de $\operatorname{rayos} \mathrm{X} \ldots \ldots \ldots \ldots \ldots \ldots$

1.5.2. Técnicas de análisis de datos de rayos X . . . . . . . . . . . . . . . . . 42

II Modelos teóricos de estrellas de neutrones $\quad 47$

2. Evolución del campo magnético en binarias de rayos $\mathrm{X} \quad \mathbf{5 1}$

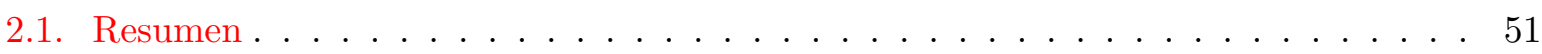

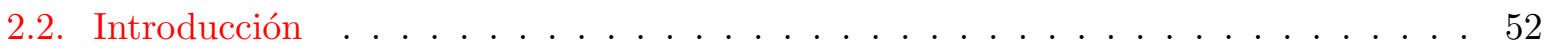

2.3. Modelo de estrella de neutrones . . . . . . . . . . . . . . . . . . . . 53

2.3.1. Corteza acretada . . . . . . . . . . . . . . . . . 53 
2.3.2. Curvas de enfriamiento . . . . . . . . . . . . . . . 54

2.3.3. Evolución del campo magnético . . . . . . . . . . . . . . . . . 55

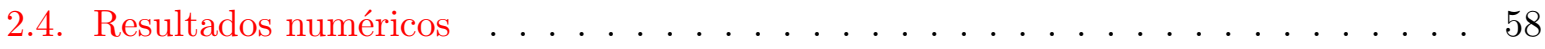

2.5. Discusión de los resultados en el contexto de los SFXTs . . . . . . . . . . . . . . 64

2.6. Conclusiones . . . . . . . . . . . . . . . . . . . . 69

3. Un modelo para el frenado del magnetar 1E $2259+586 \quad 71$

3.1. Resumen . . . . . . . . . . . . . . . . . . . . . . 71

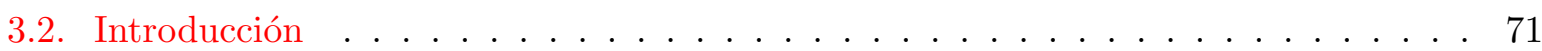

3.3. El modelo . . . . . . . . . . . . . . . . . . . . . . . . 74

3.4. Resultados . . . . . . . . . . . . . . . . . . . . 75

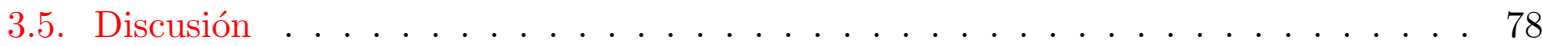

III Estudios observacionales de estrellas de neutrones $\quad 79$

4. G290.1-0.8: búsqueda de un remanente compacto 83

4.1. Resumen . . . . . . . . . . . . . . . . . . . . . 83

4.2. Introducción . . . . . . . . . . . . . . . . . . . 84

4.3. Observaciones y reducción de datos . . . . . . . . . . . . . . . 85

4.4. Resultados . . . . . . . . . . . . . . . . . . . . . 86

4.4.1. Imágenes de rayos X . . . . . . . . . . . . . . . 86

4.4.2. Análisis espectral en rayos X . . . . . . . . . . . . . . . . 87

4.4.3. Mapa de energía media de fotones . . . . . . . . . . . . . . . . . . 91

4.4.4. Análisis del medio interestelar frío a frecuencias de radio . . . . . . . . . . . 92

4.4.5. Búsqueda de fuentes puntuales en el interior del SNR G290.1-0.8 . . . . . . 94

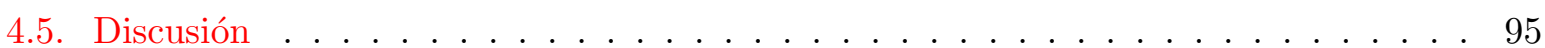

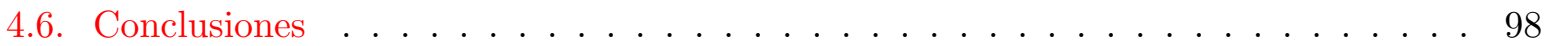

5. Enfriamiento de la estrella de neutrones en $4 \mathrm{U}$ 1820-30 99

5.1. Resumen . . . . . . . . . . . . . . . . . . . . . . . . 99

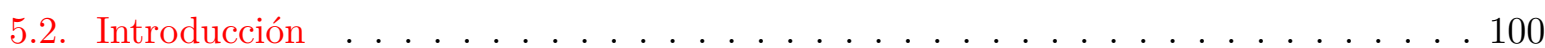

5.3. Observaciones y análisis de datos . . . . . . . . . . . . . . . . . . . 101

5.4. Resultados . . . . . . . . . . . . . . . . . . . . . . 103

5.4.1. Detección de erupciones de rayos X y análisis de diagramas de color . . . . 103

5.4.2. Análisis espectral de los estallidos de rayos X . . . . . . . . . . . . . . . 104

5.4.3. Relación flujo-temperatura durante la fase de enfriamiento . . . . . . . . . . 107

5.5. Discusión . . . . . . . . . . . . . . . . . . . . . 109 
IV Estrellas de neutrones en gravedad modificada

6. Gravedad cuadrática $f(R)$ en el formalismo métrico $\quad 117$

6.1. Resumen . . . . . . . . . . . . . . . . . . . . . 117

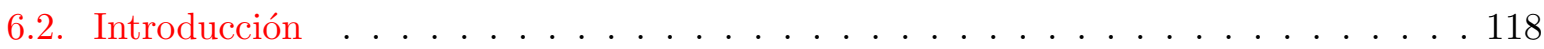

6.3. Ecuaciones de la estructura estelar . . . . . . . . . . . . . . . . . . . . . 119

6.4. Ecuaciones de estado . . . . . . . . . . . . . . . . . . . . 121

6.5. Método numérico . . . . . . . . . . . . . . . . . . . . . . 122

6.6. Resultados . . . . . . . . . . . . . . . . . . . . . 123

6.7. Discusión . . . . . . . . . . . . . . . . . . . . 128

7. Gravedad cuadrática $f(R)$ en el formalismo de Palatini $\quad 131$

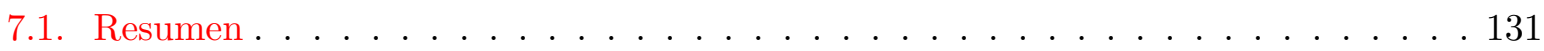

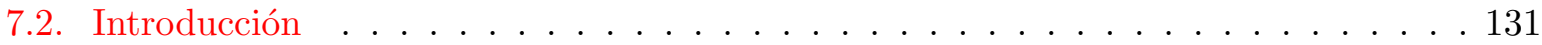

7.3. Ecuaciones de la estructura estelar . . . . . . . . . . . . . . . . . . 133

7.4. Ecuación de estado . . . . . . . . . . . . . . . . . . . 135

7.5. Método numérico . . . . . . . . . . . . . . . . 136

7.6. Resultados . . . . . . . . . . . . . . . . . . . . . 137

7.7. Una parametrización alternativa . . . . . . . . . . . . . . . 138

7.8. Discusión . . . . . . . . . . . . . . . . . . . . . . 142

V Conclusiones y perspectivas a futuro $\quad 143$

8. Conclusiones y desarrollos a futuro $\quad 145$

$\begin{array}{ll}\text { Bibliografía } & 154\end{array}$ 
Parte I

\section{Introducción}





\section{Capítulo 1}

\section{Estrellas de neutrones}

Las estrellas de neutrones $\left(\mathrm{NSs}^{1}\right)$ son los objetos materiales más compactos que se conocen. Las condiciones extremas a la que están sometidos el espacio y la materia, vuelven a estos objetos únicos y fascinantes desde el punto de vista de la astrofísica relativista. La imposibilidad de reproducir estas condiciones en experimentos controlados, convierte a estas estrellas en laboratorios cruciales para comprender las propiedades fundamentales de la naturaleza de la materia a las más altas densidades. A su vez, los campos gravitatorios involucrados son tan intensos que tornan a estas estrellas totalmente degeneradas y relativistas, a punto tal que podrían ser escenarios favorables para poner a prueba diferentes teorías de gravedad. La existencia de una masa máxima para estos objetos y la determinación de su magnitud, así como la de su tamaño asociado resulta uno de los principales desafíos para las observaciones, para lo cual resulta determinante comprender las condiciones astrofísicas impuestas por los entornos en que se hallan inmersas.

En este primer capítulo, se realiza un breve repaso histórico sobre los primeros modelos teóricos desarrollados hasta el descubrimiento de las NSs y dando un enfoque del conocimiento actual acerca de estos objetos, destacando los puntos donde esta tesis realiza aportes como producto de los resultados presentados en los capítulos subsiguientes.

\subsection{Historia}

A partir de que Chadwick (1932) anunciara el descubrimiento de un nucleón neutro en el año 1932, las especulaciones acerca de la posibilidad de la existencia de estrellas formadas esencialmente por neutrones no tardaron en aparecer, despertando interés en la comunidad astrofísica. En 1933, Baade \& Zwicky (1934) propusieron la existencia de lo que denominaron NSs. En ese breve trabajo plantearon que estas estrellas se formarían como producto de las ya conocidas explosiones de supernova, prediciendo radios pequeños y densidades extremadamente grandes como sus principales características. Unos años después, Oppenheimer \& Volkoff (1939) y, de manera independiente, Tolman (1939), derivaron las ecuaciones de equilibrio hidrostático para estrellas

\footnotetext{
${ }^{1}$ Del inglés, Neutron Stars.
} 
esféricas en el marco de la teoría general de la relatividad $\left(\mathrm{GR}^{2}\right)$. Proponiendo que la materia en su interior consiste básicamente de un gas de neutrones degenerados relativistas, construyeron los primeros modelos y configuraciones de NSs, obteniendo para la masa máxima un valor de $\sim 0.71 \mathrm{M}_{\odot}$ y radios de tan sólo $\sim 10 \mathrm{~km}$.

Dado el pequeño tamaño asociado a la superficie predicha para estas estrellas, parecía muy poco probable poder detectar emisión térmica proveniente de su superficie, sin embargo, a partir de 1962, con el advenimiento de la primera generación de satélites de rayos X, se realizaron los primeros intentos por dar con estos objetos. Poco tiempo después, el grupo liderado por Giacconi et al. (1962) descubrió varias fuentes compactas de rayos X, pero la conexión con las NSs no pudo ser establecida.

Unos pocos años después, en 1967, en el otro extremo del espectro electromagnético y de manera totalmente casual, J. Bell descubrió la emisión periódica en ondas de radio de lo que su director, A. Hewish, llamara pulsares. Ese mismo año, Pacini (1967) propuso que NSs con grandes velocidades de rotación y fuertes campos magnéticos, transformarían inevitablemente su energía rotacional en radiación electromagnética, acelerando partículas cargadas hasta velocidades relativistas. Al año siguiente, Hewish et al. (1968) publicó el descubrimiento de los pulsares y (Gold, 1968) sugirió que la emisión periódica detectada por los radiotelescopios era consecuencia del mecanismo propuesto por Pacini, asociando el descubrimiento de Hewish a la primera observación de una NS. Hacia fines de ese año, Comella et al. (1969) consiguieron detectar un pulsar en la mismísima Nebulosa del Cangrejo, o Crab, remanente de una supernova registrada por astrónomos chinos en el año 1054. Con un período de $33 \mathrm{~ms}$, la emisión no podía ser explicada por la rotación de una estrella enana blanca y debía estar asociada a una NS. De esta manera, la vieja predicción de Baade y Zwicky fue confirmada por las observaciones.

Gracias a que los pulsos de radiación son intensos y regulares, los astrónomos pueden determinar su período con muchísima precisión. A su vez, la medición regular del período, permitió notar que los pulsares están frenándose, ya que sus períodos crecen con el tiempo. La rápida variación del campo magnético produce la emisión electromagnética descripta que disminuye la energía rotacional de la estrella, frenándola con una tasa típica del orden de $10^{-15} \mathrm{~s}$ en cada vuelta. Esta variación lenta pero continua, permitió descartar la posibilidad de que los pulsos detectados fueran originados por oscilaciones en la estructura del objeto, razón por la cual fueron denominados pulsares.

Si bien todas las estrellas normales poseen un campo magnético, estos son relativamente débiles. Durante una explosión de supernova, el núcleo estelar debe ser comprimido para formar una NS. A medida que las líneas de campo magnético se aproximan, su intensidad se incrementa fuertemente. El campo magnético formado, combinado con la rotación, provoca corrientes eléctricas enormes en la superficie de la NS. Esto provoca una pérdida de electrones (y positrones) en las proximidades de los polos magnéticos de la estrella que fluyen a lo largo de las líneas de campo magnético produciendo radiación sincrotrón y de curvatura. Así, las NSs tienen entonces dos fuentes de radiación, por un lado emisión electromagnética no térmica producida por las par-

\footnotetext{
${ }^{2}$ Del inglés, General Relativity.
} 
tículas atrapadas en las líneas de campo magnético de la estrella, predominante en frecuencias de radio, y por otro lado, radiación térmica debida a partículas que colisionan con la superficie de la estrella en los polos magnéticos, detectable incluso en los rayos X.

Como el eje magnético de la NS no coincide necesariamente con el de rotación, pudiendo estar inclinado, para un observador lejano, esta radiación resulta pulsada, como en el caso de un faro, y sólo es detectable en el caso en que el eje magnético coincida con la dirección del observador durante el giro (ver Figura 1.1).

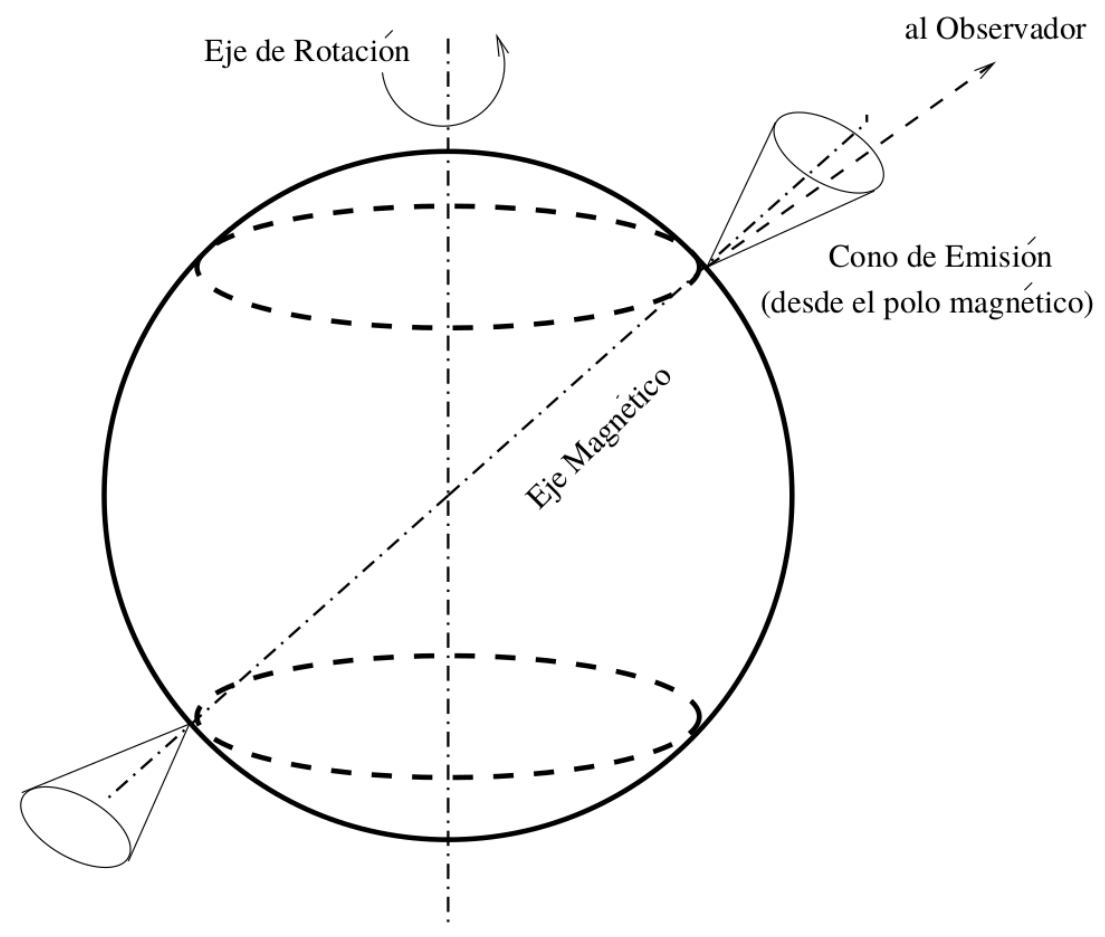

Figura 1.1: Esquema de un pulsar.

Paralelamente, Shkolvsky (1968) planteó la posibilidad de la existencia de sistemas binarios compuestos por una NS capaz de acretar materia proveniente de la envoltura de una estrella normal. El mecanismo propuesto sería entonces capaz de liberar una gran cantidad de energía en el rango de los rayos X. Ya en los años 70, a partir de las observaciones del satélite de rayos X UHURU, bajo esta hipótesis, fue posible establecer la relación entre una serie de fuentes compactas brillantes y las NSs. La presencia de NSs en sistemas binarios permitió, además, obtener las primeras medidas de sus masas. Unos años después, Hulse \& Taylor (1974) descubrieron el primer pulsar binario (PSR B1913+16) y con él consiguieron, no sólo determinar las masas de ambas NSs, sino también estimar la radiación gravitacional emitida por el sistema, en total acuerdo con las predicciones de GR.

Ya a fines de la década de los 70s, se obtuvieron las primeras imágenes de alta resolución espacial en rayos X, usando el instrumento Einstein, lo que posibilitó comprender la morfología extendida de los restos de explosiones de supernova y su asociación con las NSs. Más adelante, 
en la década del 90, el satélite ROSAT, con una sensibilidad mil veces mayor que UHURU, detectó una gran cantidad de nuevas fuentes y, a partir de 1995, con el advenimiento del satélite RXTE se obtuvieron nuevas curvas de luz de estos objetos, alcanzando escalas temporales de la millonésima parte de un segundo, lo que posibilitó estudiar el comportamiento de la materia en las proximidades de estos objetos y hasta en su propia superficie.

A partir de 1999 fueron puestos en funcionamiento los satélites de rayos X Chandra y XMMNewton. Estos observatorios incorporaron superficies ópticas usando el sistema diseñado por Wolter, convirtiéndose así en verdaderos telescopios de rayos $\mathrm{X}$, con grandes mejoras respecto a sus antecesores. Con mayores resoluciones, tanto espaciales, temporales como espectrales, pueden no sólo obtener imágenes, sino también tomar espectros y curvas de luz extremadamente precisos, simultáneamente, que permitieron llevar a cabo una clasificación más detallada de las NSs generando nuevos desafíos teóricos.

Las NSs son objetos muy especiales, ya que su estudio requiere la incorporación de herramientas de diversas ramas de la física y la astronomía que incluyen a la física de partículas, nuclear, la magnetohidrodinámica de plasmas, gravitación, y procesos radiativos que abarcan desde emisión en radio, hasta rayos $\mathrm{X}$ y $\gamma$, así como emisión de neutrinos y ondas gravitacionales. En particular, la densidad de la parte más interna de estos objetos exige el tratamiento de la materia nuclear en un continuo y a baja temperatura, o incluso podría involucrar la existencia de otras fases de materia extraña como un gas de quarks con interacciones entre ellos. Estas condiciones son imposibles de conseguir en los laboratorios terrestres, por lo cual las NSs resultan laboratorios naturales únicos para el estudio de la materia a densidades extremas y el régimen de campo gravitacional fuerte para la curvatura del espacio-tiempo.

\subsection{Características generales de las estrellas de neutrones}

En una estrella normal, la presión de la materia y la radiación en el interior equilibran la fuerza ejercida por su propio campo gravitatorio. La energía que produce la radiación es proporcionada por las reacciones nucleares que ocurren en las partes más internas, o núcleo de la estrella. Cuando el combustible de estas reacciones se agota, éstas cesan y el equilibrio se rompe, provocando el ingreso a una nueva etapa de la propia estrella que parafraseamos como el fin de su vida. Aquí, los caminos se bifurcan dependiendo esencialmente de la masa de la estrella. Si la estrella tiene una masa $M<8 \mathrm{M}_{\odot}$, su atmósfera se expandirá lentamente formando una nebulosa planetaria mientras que su núcleo se comprimirá hasta alcanzar una nueva forma de equilibrio, gracias a la presión de degeneración de los electrones, convirtiéndose en una estrella enana blanca. Si la estrella es de gran masa $\left(M>20 \mathrm{M}_{\odot}\right)$, explotará como una supernova mientras que la presión de la materia en su núcleo no será suficiente para soportar la atracción gravitacional, por lo que éste colapsará, formando un agujero negro. En cambio, si la estrella es de masa intermedia $\left(8 \mathrm{M}_{\odot}<M<20 \mathrm{M}_{\odot}\right)$, si bien también explotará en una supernova, su núcleo, en cambió podrá encontrar una nueva configuración de equilibrio frente a la gravedad, proporcionada por la presión de un gas de neutrones degenerado, lo que da el nombre de NS. 


\subsubsection{Propiedades físicas macroscópicas}

Las NSs constituyen los objetos estelares más compactos que se conocen, con masas $M$ $1.4 \mathrm{M}_{\odot} \mathrm{y}$ radios $R \sim 10 \mathrm{~km}$, su densidad promedio resulta:

$$
\bar{\rho}=3 M /\left(4 \pi R^{3}\right) \approx 7 \times 10^{14} \mathrm{gr} \mathrm{cm}^{-3} \sim 2.5 \rho_{0}
$$

donde $\rho_{0} \sim 2.8 \times 10^{14} \mathrm{gr} \mathrm{cm}^{-3}$ es la densidad de saturación nuclear, que es la densidad de equilibrio de los nucleones dentro de los núcleos atómicos. A diferencia de otros objetos compactos como las enanas blancas, en las cuales las aproximaciones newtonianas son válidas (Shapiro \& Teukolsky, 1983), las densidades involucradas implican un campo gravitacional tan intenso que para modelarlas resulta necesario un tratamiento relativista. Un simple cálculo permite determinar que el corrimiento al rojo en su superficie es $z=\left(1-2 G M / R c^{2}\right)^{-1 / 2}-1 \approx 0.1 \mathrm{y}$, por lo tanto, el efecto del campo gravitatorio sobre los fotones emitidos, no puede ignorarse. Hasta el presente, entre la Vía Láctea y las Nubes de Magallanes, se han detectado unas dos mil NSs, de las cuales la gran mayoría se manifiestan como pulsares. La población está concentrada fuertemente en el disco de la galaxia, aunque con una cierta dispersión hacia latitudes más altas (Lattimer \& Prakash, 2007).

Las NSs heredan al menos dos propiedades fundamentales de su estrella progenitora: la rotación y el campo magnético. Por un lado, la conservación del momento angular $L=I \Omega$ hace que las NSs, cuyos radios son mucho menores que los de sus estrellas progenitoras $\left(\sim 10^{5} \mathrm{~km}\right) \mathrm{y}$, por lo tanto, momentos de inercia, $I$, muy inferiores, tengan asociadas frecuencias de rotación $\Omega$ muchísimo mayores a sus progenitoras. De un mismo modo, la conservación del flujo magnético $\phi \sim B / 4 \pi r^{2}$ amplifica un campo magnético típico de $B \sim 10^{4} \mathrm{G}$ asociado a las estrella originales, hasta valores $B \sim 10^{12} \mathrm{G}$ para las NSs.

\subsection{2. $\quad$ El diagrama $P-\dot{P}$}

Como vimos las NSs están asociadas a altas velocidades de rotación, con períodos que van desde el milisegundo hasta la decena de segundos. En efecto, en el caso de los pulsares, su radiación no térmica, visible en ondas de radio, es obtenida a expensas de su energía rotacional $E_{\text {rot }}=$ $I \Omega^{2} / 2$. Esta radiación disminuye la energía rotacional de la estrella por lo que su período de rotación aumenta $(\mathrm{d} P / \mathrm{d} t \dot{P}>0)$. Por ejemplo, para el caso del pulsar del Crab $(P=33 \mathrm{~ms}$ y $\left.\dot{P}=4.22 \times 10^{-13} \mathrm{~s} \mathrm{~s}^{-1}\right), \dot{E}_{\text {rot }}=I \Omega \dot{\Omega} \sim 5 \times 10^{38} \mathrm{erg} \mathrm{s}^{-1}$ que coincide con la luminosidad detectada en radio. Considerando que esta radiación proviene de un dipolo magnético en rotación, la intensidad del campo magnético puede estimarse con la siguiente ecuación (Shapiro \& Teukolsky, 1983):

$$
B=\left(\frac{3 I c^{3}}{8 \pi^{2} R^{6}} P \dot{P}\right)^{1 / 2} \Rightarrow B \approx 3.2 \times 10^{19}(P \dot{P})^{1 / 2} \mathrm{G}
$$

donde $c$ es la velocidad de la luz. Bajo estas condiciones, conocidos $P$ y $\dot{P}$ para un pulsar determinado, y suponiendo que el período inicial es muy bajo, puede también estimarse una edad característica de la NS con la siguiente relación: $\tau \sim P / 2 \dot{P}$. 


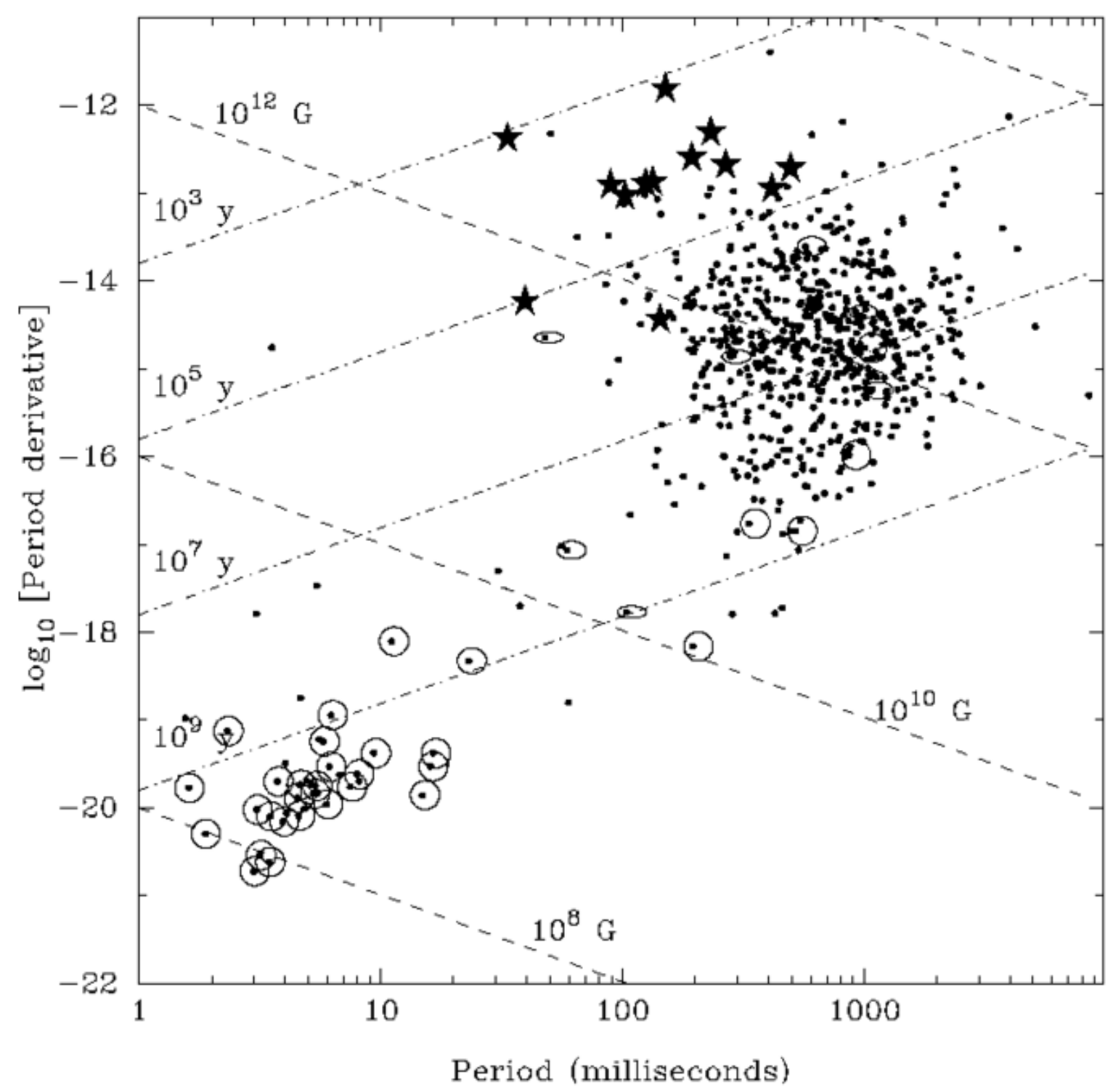

Figura 1.2: Diagrama $P-\dot{P}$ de algunos pulsares conocidos (Lorimer, 2001). En las abscisas se grafica el período $P[\mathrm{~ms}]$ y en las ordenadas el logaritmo de la derivada del período $\dot{P}\left[\mathrm{~s} \mathrm{~s}^{-1}\right]$. Las líneas a trazos representan intensidad de campo magnético constante mientras que las líneas punteadas con trazos, corresponden a edad característica $\tau$ constante en años. Los pulsares en sistemas binarios se encuentran resaltados por círculos o elipses según la excentricidad del sistema. Los pulsares (jóvenes) asociados a SNRs son representados por estrellas de cinco puntas.

De este análisis resulta esperable una correlación entre el período $P$ y su derivada $\dot{P}$, que se confirma en la disposición de las NSs conocidas sobre el diagrama $P-\dot{P}$, presentado en la Figura 1.2 (Lorimer, 2001). Allí se observa que los pulsares se dividen en dos grandes grupos. Por un lado, en la zona superior derecha del diagrama, donde se concentra la gran mayoría de estos objetos, 
encontramos NSs con campos magnéticos $B \sim 10^{12} \mathrm{G}$ y edades características $\tau \lesssim 10^{7-8}$ años. Como dijimos, a medida que envejecen, los pulsares se van frenando, por lo que se mueven en el diagrama hacia la derecha y abajo. Este frenado, a su vez, disminuye la actividad magnetosférica de la NS, hasta que finalmente el pulsar puede apagarse completamente, lo que da origen a la región denominada graveyard o cementerio, en el extremo inferior derecho del diagrama. Por otro lado, en la región inferior izquierda se encuentran los pulsares del milisegundo, asociados en su gran mayoría a sistemas binarios, y con campos magnéticos más bajos $\left(B \sim 10^{8-9} \mathrm{G}\right)$. Se piensa que estos pulsares son reciclados de la zona inferior derecha del diagrama debido a que un prolongado proceso de incorporación de materia y momento angular a partir de un disco de acreción que reactiva su actividad.

La evolución de las NSs también se manifiesta a través de un largo proceso de enfriamiento que se evindencia ya que si bien al formarse, como producto de la violenta explosión de supernova que da lugar a su origen, las NSs tienen temperaturas internas muy altas $\left(T \gtrsim 10^{9} \mathrm{~K}\right)$, NSs más viejas, en cambio, se encuentran a temperaturas mucho más bajas del orden de $T \sim 10^{5-6} \mathrm{~K}$ (Yakovlev et al., 2001). Si bien el máximo de la radiación térmica asociada a esta temperatura, se encuentra en la región de los rayos X, en algunos casos, la radiación también puede detectarse en el ultravioleta o incluso en el óptico (Walter \& Matthews, 1997).

\subsubsection{Campo magnético y clasificación, desde magnetares hasta $C C O s$}

Las NSs poseen campos magnéticos varios órdenes de magnitud más intensos que las estrellas normales. A su vez, como vimos en la sección anterior, éstos abarcan varias décadas en intensidad como el caso de los pulsares normales, y los pulsares del milisegundo. El amplio rango de intensidades del campo magnético asociado a las NSs conlleva la manifestaciones fenomenológicas absolutamente diferentes. Es por esto que este parámetro resulta un buen ordenador a la hora de clasificar esta estrellas, abarcando (Kaspi, 2010): pulsares alimentados por rotación (RPPs ${ }^{3}$, con $B \sim 10^{12} \mathrm{G}$ ), pulsares del milisegundo $\left(\mathrm{MSPs}^{4}\right.$, con $\left.B \sim 10^{8} \mathrm{G}\right)$, estrellas aisladas débiles en rayos $\mathrm{X}$ (X-dim $\mathrm{INS}^{5}$, con $B \sim 10^{13} \mathrm{G}$ ), pulsares de campo magnético alto (High-B RPP, con $B \sim 10^{14} \mathrm{G}$ ) con gran actividad en altas energías, repetidores de rayos $\gamma$ suaves (SGRs ${ }^{6}$ ) y pulsares anómalos de rayos $\mathrm{X}\left(\mathrm{AXPs}^{7}\right)$, también llamados magnetares, con campos magnéticos muy intensos, $B \gtrsim 10^{14} \mathrm{G}$. Más recientemente se ha propuesto además la catergoría de los objetos compactos centrales en remanentes de supernova $\left(\mathrm{CCOs}^{8}\right.$ en $\left.\mathrm{SNRs}^{9}\right)$, con $B \lesssim 10^{11} \mathrm{G}$.

En el Capítulo 4 de la Parte III presentaremos un estudio detallado de la emisión extendida de un SNR que podría albergar un objeto compacto (como un CCO) en su interior. Para ello, incorporaremos datos obtenidos por los satélites XMM-Newton y Chandra que presentaremos

\footnotetext{
${ }^{3}$ Del inglés, Rotation Powered Pulsars.

${ }^{4}$ Del inglés, Milli-Second Pulsars.

${ }^{5}$ Del inglés, X-ray dim Isolated Neutron Stars.

${ }^{6}$ Del inglés, Soft Gamma-ray Repeaters.

${ }^{7}$ Del inglés, Anomalous X-ray Pulsars.

${ }^{8}$ Del inglés, Central Compact Objects.

${ }^{9}$ Del inglés, Supernova Remnants.
} 
hacia el final de este capítulo.

En general, la intensidad del campo magnético es inferida a través de observaciones indirectas. Por ejemplo, si de las observaciones se determinan $P$ y $\dot{P}$, el campo puede estimarse a partir de la Ecuación 1.2. En algunos casos, el campo magnético también puede derivarse de mediciones en la banda de los rayos X, a través de la detección de líneas de emisión ciclotrón en los espectros (Gnedin \& Sunyaev, 1974; Trümper et al., 1978). Las observaciones indican, en muchos casos, que existe una correlación inversa entre el campo magnético exterior y la edad de la NS. Los campos magnéticos de NSs asociadas a SNRs ( $\leqslant 10^{5}$ años), son varios órdenes de magnitud mayores a los de aquellas que pertenecen a sistemas binarios de baja masa, donde la edad de las NSs allí alojadas debe ser mucho mayor ( $\sim 0^{8-9}$ años). Este efecto evidencia la existencia de un proceso evolutivo que podría estar dominado por diferentes factores. En la corteza, el efecto más importante es la difusión óhmica (Geppert \& Urpin, 1994; Urpin \& Geppert, 1995) y el calentamiento por efecto Joule (Pons et al., 2007). Este proceso provoca un calentamiento de la corteza, por lo que el enfriamiento de las NSs se ve alterado por la evolución magnética lo que sugiere una fuerte correlación entre ambas (Aguilera et al., 2008; Pons et al., 2009). El proceso acoplado de evolución magnéto-térmica fue estudiado recientemente por Viganò et al. (2013). La acreción de material del entorno produce un aumento local de la temperatura en las capas externas donde el material acretado libera energía gravitacional y da lugar a una compresión del material que deriva en reacciones termonucleares inestables que provocan erupciones termonucleares, brillantes en los rayos X. El nuevo material y su catalización, generan impurezas en la corteza, aumentando la resistividad y, por ende, aceleran el decaimiento del campo magnético (Battacharya, 2002). En el Capítulo 2 de la Parte II estudiaremos estos procesos en detalle aplicando un modelo de evolución del campo magnético a una NS que acreta materia proveniente del viento de una estrella compañera supergigante en un sistema binario.

\subsubsection{Estructura interna}

La estructura de las NSs se divide en cuatro capas: atmósfera, envoltura, corteza y núcleo (Haensel et al., 2007).

La atmósfera es una capa delgada de unos $0.1-10 \mathrm{~cm}$ de espesor. Está compuesta por un plasma similar al de una fotosfera estelar, que modela el espectro de la radiación térmica detectable, dependiendo de la temperatura y gravedad superficiales, la composición química y de la intensidad y geometría del campo magnético. Bajo esta atmósfera se encuentra la envoltura que está compuesta esencialmente por un plasma de iones y un gas de electrones degenerados. A partir de densidades $\rho \sim 10^{4} \mathrm{~g} \mathrm{~cm}^{-3}$, los átomos se ionizan completamente y comportándose como un gas libre de Boltzmann. En cambio, a densidades $\rho \sim 10^{6} \mathrm{gr} \mathrm{cm}^{-3}$, su interacción crece y forman un líquido comúnmente denominado océano. Más allá de densidades $\rho \sim 10^{6} \mathrm{gr} \mathrm{cm}^{-3}$, el gas de electrones degenerados se vuelve ultra-relativista.

La corteza se extiende desde la densidad de solidificación, $\rho \sim 10^{8-9} \mathrm{gr} \mathrm{cm}^{-3}$, hasta la aparición del núcleo de la estrella, a $\rho \sim 10^{14} \mathrm{gr}^{-3}$. Tiene un espesor típico del orden de $\sim 1 \mathrm{~km}$ y está dividida en una zona externa y otra interna. 


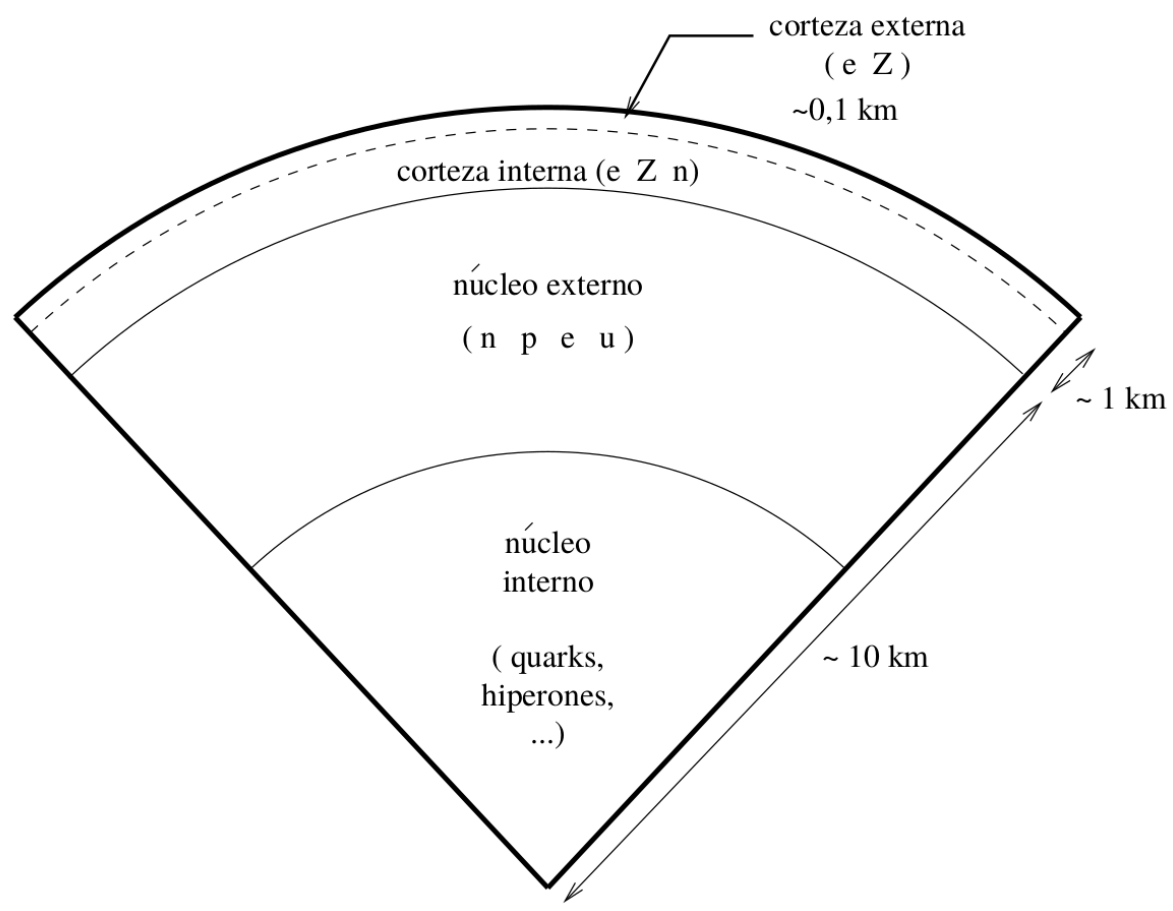

Figura 1.3: Representación esquemática de la estructura de una NS adaptada de Haensel et al. (2007).

La corteza externa tiene espesores típicos de $0.1 \mathrm{~km}$ y está conformada por una red cristalina de iones con número atómico $Z$ creciente hacia el interior y un gas de electrones degenerado relativista que proporciona la presión en esta región. Hacia el interior, a medida que la densidad aumenta, la captura electrónica por parte de los iones $\left(p+e^{-} \rightarrow n+v\right)$ enriquece en neutrones los núcleos hasta que, en la interfase con la corteza interna, a la densidad $\rho_{\mathrm{ND}} \sim 4 \times 10^{11} \mathrm{~g} \mathrm{~cm}^{-3}$, los neutrones comienzan a desligarse de $\operatorname{los}$ núcleos ${ }^{10}$, formando un gas de neutrones libres que coexiste con la red cristalina.

La corteza interna, con un espesor $\sim 1 \mathrm{~km}$, se extiende desde la densidad $\rho_{\mathrm{ND}}$ hasta $\rho \sim$ $0.5 \rho_{0}=1.4 \times 10^{14} \mathrm{gr} \mathrm{cm}^{-3}$. A medida que la densidad aumenta hacia el interior, la fracción de neutrones libres se va incrementando hasta que en la base de la corteza, la interacción repulsiva de corto alcance entre los neutrones se torna lo suficientemente importante como para influenciar a los neutrones colectivamente. Allí, los neutrones libres pueden presentar interacciones atractivas entre sí que, aunque débiles, derivan en la formación de un estado superfluido, similar a la superfluidez del ${ }^{3} \mathrm{He}$ (Shapiro \& Teukolsky, 1983).

Por último, el núcleo también se divide en una parte externa $\left(0.5 \rho_{0} \lesssim \rho \lesssim 2 \rho_{0}\right)$, que está presente en todas las NSs, y una parte interna $\left(\rho \gtrsim 2 \rho_{0}\right)$ que sólo es contenida por las NSs más masivas. La parte externa, de unos kilómetros de espesor, está compuesta por materia nuclear constituida por un gas eléctricamente neutro de protones, neutrones, electrones y, eventualmente,

\footnotetext{
${ }^{10} \mathrm{~A}$ este fenómeno se le denomina goteo de neutrones (en inglés, neutron drip, de donde surge $\rho_{\mathrm{ND}}$ ).
} 
muones en equilibrio beta $\left(p+e^{-} \rightarrow n+v_{e}\right.$ y $\left.n \rightarrow p+e^{-}+\overline{v_{e}}\right)$. En el caso de los protones, como se trata de partículas cargadas, pueden volverse superconductores.

La región más interna, que también se extiende varios kilómetros, puede alcanzar en su centro densidades extremas $\rho \sim 10 \rho_{0}$. La naturaleza de la materia a estas densidades es totalmente desconocida. Entre las posibilidades discutidas en la literatura, podemos mencionar: la presencia de hiperones, condensados de piones, kaones o incluso una transición de fase donde los quarks se desligan de los núcleos atómicos (Glendenning, 2000).

\subsubsection{Anomalías en la rotación: glitches y anti-glitches}

En algunos pulsares el frenado lento que sufren a lo largo de toda su vida, es alterado por la presencia de leves aceleraciones repentinas en el orden de segundos a minutos de duración. Estas anomalías o glitches que aparecen en su curvas temporales de rotación son seguidas por un período de relajación parcial hasta que recuperan su frenado natural, en escalas de tiempo de días, meses o hasta años. Los cambios relativos en frecuencia pueden variar desde $\Delta v / v \sim 10^{-10}$ hasta $5 \times 10^{-6}$, habiendo sido detectadas unas 315 anomalías en unos 102 pulsares (Espinoza et al., 2011). Estas anomalías son observadas principalmente en pulsares relativamente jóvenes, y las primeras detecciones se realizaron justamente en el Crab (Boynton et al., 1969) y el pulsar de Vela (Radhakrishnan \& Manchester, 1969), que ha experimentado un total de 16 glitches desde 1969, todas del orden de $10^{-6}$ de magnitud (Espinoza et al., 2011). Si bien la mayoría de las detecciones son producto de un minucioso monitoreo en ondas de radio, estos fenómenos también han sido detectados en seguimientos de fuentes brillantes en los rayos $\mathrm{X}$.

A pesar de que el mecanismo responsable de desatar estas anomalías no se conoce a fondo, varios modelos han sido propuestos para explicarlas, desarrollados únicamente a partir dos ideas básicas. Como la intensidad de la gravedad en la superficie de la NS es muy fuerte (varios órdenes de magnitud mayor a la terrestre), el peso de la corteza provoca un re-acomodamiento de la estrella a medida que ésta se frena, liberando la energía acumulada en las tensiones a la que es sometida la corteza. La contracción, aunque milimétrica, da como resultado un desplazamiento que modifica levemente la velocidad de rotación, fenómeno al que se accede gracias a la extrema precisión con que es determinada la velocidad de rotación y su evolución (Baym \& Pines, 1971; Ruderman, 1969). En cambio, Anderson \& Itoh (1975) propusieron que estas anomalías podrían tener origen en una transferencia repentina de momento angular desde un superfluido que rota rápidamente en el interior de la estrella, a una corteza sólida que lo hace más lentamente. Esta componente superfluida yacería en las regiones internas donde los vórtices de neutrones, que acarrean el momento angular del superfluido, no pueden moverse por estar fijos a los iones de la corteza. Como, en esas condiciones, el superfluido no puede frenarse, se mantiene a una velocidad de rotación mayor que el resto de la estrella que sí se frena a medida que su energía rotacional es radiada. Así, la anomalía podría ocurrir cuando estos vórtices se desacoplan momentáneamente de la corteza, viajando hacia el exterior y permitiendo un rápido intercambio del momento angular, que acelera la corteza hasta acoplarse nuevamente.

Sin embargo, recientemente, Archibald et al. (2013) detectaron el fenómeno contrario: un 
frenado repentino ocurrido en un magnetar que difícilmente podría ser explicado por este mecanismo. En el Capítulo 3 de la Parte II presentaremos un modelo, que podría dar cuenta de esta anomalía, en el marco de la evolución del campo magnético de NSs altamente magnetizadas.

\subsubsection{Estrellas de neutrones en sistemas binarios}

Del conjunto de más de 2500 NSs detectadas hasta hoy, aproximadamente, unas 300 se encuentran en sistemas binarios (Manchester et al., 2005), donde las estrellas compañeras abarcan desde estrellas normales con diferentes masas, hasta enanas blancas u otras NSs, como el pulsar binario estudiado por Hulse \& Taylor (1974).

Si la NS se encuentra acretando materia de su compañera, los sistemas se tornan brillantes en los rayos $\mathrm{X}$ y se denominan sistemas binarios de rayos $\mathrm{X}\left(\mathrm{XRBs}^{11}\right)$. La radiación emitida puede originarse en la superficie de la NS o cerca de ella (debido a que la materia que cae en el campo gravitacional de la NS, emite radiación con energías del orden de los keV), o bien en el disco de acreción donde $T \sim 10^{6-7} \mathrm{~K}$. En general, la energía liberada producto de la acreción puede estimarse como:

$$
\dot{E} \approx \frac{G M \dot{M}}{R} \approx 8 \times 10^{35}\left(\frac{\dot{M}}{10^{-10} \mathrm{M}_{\odot} \mathrm{año}^{-1}}\right)\left(\frac{M}{\mathrm{M}_{\odot}}\right)\left(\frac{R}{10 \mathrm{~km}}\right) \mathrm{erg} \mathrm{s}^{-1}
$$

donde $G$ es la constante de gravitación universal, $M$ y $R$ son la masa y el radio de la NS, respectivamente, y $\dot{M}$ es la tasa de acreción.

En particular, si la estrella donante tiene una masa $M \lesssim 1 \mathrm{M}_{\odot}$, el sistema se denomina sistema binario de rayos X de baja masa $\left(\mathrm{LMXB}^{12}\right)$, mientras que, si la estrella donante tiene $M \gtrsim 10 \mathrm{M}_{\odot}$, el sistema se denomina sistema binario de rayos $\mathrm{X}$ de alta masa $\left(\mathrm{HMXB}^{13}\right)$. Actualmente se conocen del orden de 180 LMXBs y 110 HMXBs (Liu et al., 2006).

En el caso de los LMXBs, la acreción de materia sobre la NS ocurre cuando, al evolucionar, la estrella compañera ocupa todo su lóbulo de Roche. Luego, la materia de su envoltura comienza a ser transferida a través del punto de Lagrange, formando un disco de acreción en torno a la NS. Como estas estrellas tardan miles de millones de años en evolucionar de la secuencia principal, las NSs en estos sistemas suelen tener edades $\sim 10^{8-9}$ años, y campos magnéticos bajos $\left(B \sim 10^{8-9} \mathrm{G}\right)$. Algunos de estos sistemas son capaces de acelerar partículas hasta velocidades relativistas, colimándolas a través de torres magnéticas, en lo que se denominan jets. La Figura 1.4 muestra el esquema de una NS acretando materia de un disco de acreción. A diferencia de lo que ocurre con un agujero negro, la materia de la zona más interna del disco es canalizada a través de las líneas del campo magnético de la NS y la acreción se produce principalmente a través de los sus polos magnéticos. Allí se produce el choque de este flujo de materia con la superficie de la NS, generando una mancha caliente (hotspot) detectable en la banda de rayos X. Es importante notar que la tasa de acreción local, generalmente expresada en $\mathrm{g} \mathrm{s}^{-1}$ puede diferir de la tasa de

\footnotetext{
${ }^{11}$ Del inglés, X-ray Binaries.

${ }^{12}$ Del inglés, Low-Mass X-ray Binary.

${ }^{13}$ Del inglés, High-Mass X-ray Binary.
} 


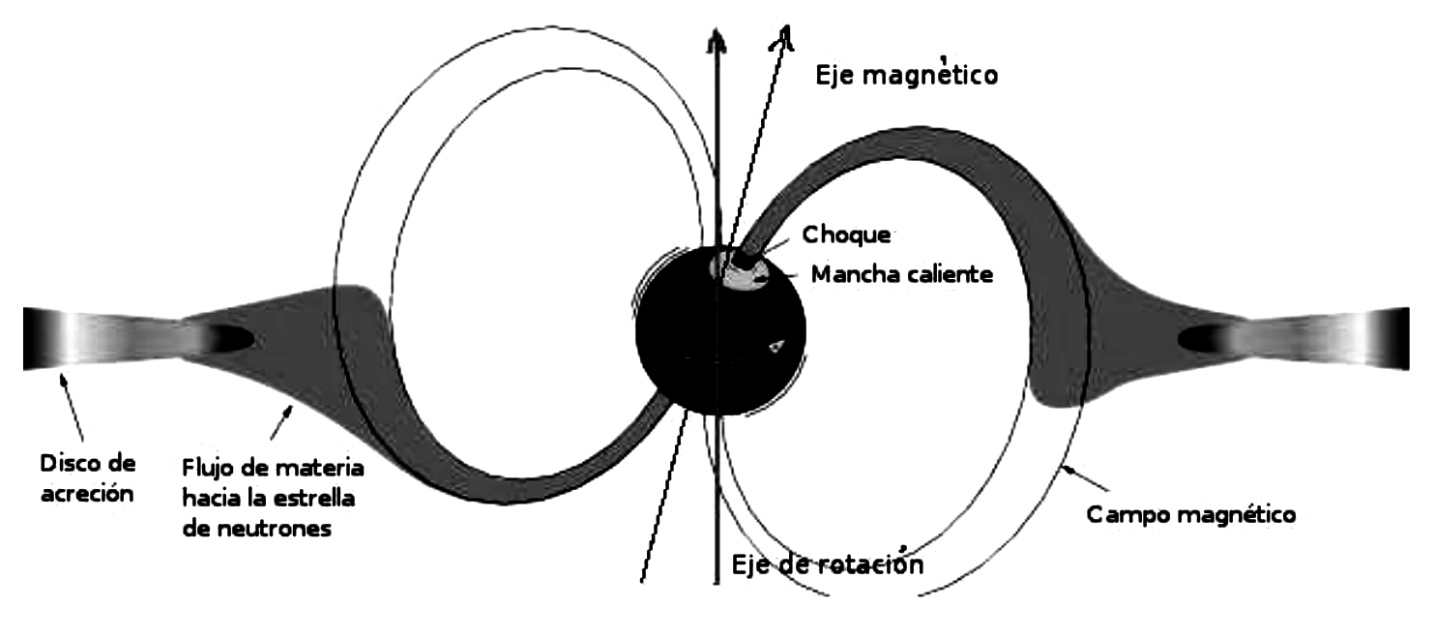

Figura 1.4: Esquema de una NS acretando materia proveniente de un disco de acreción.

acreción global, promediada sobre toda la superficie de la estrella, si la acreción no es esféricamente simétrica, como en este caso.

En el caso de los HMXBs, las estrellas donantes son de tipo temprano, con tipos espectrales $\mathrm{O}$ o B, y presentan vientos intensos. Una fracción de estos vientos es atraída hasta la NS, que incluso siendo muy joven, experimenta un proceso de acreción, aunque mucho más leve que en los LMXBs. Como las estrellas de gran masa evolucionan rápidamente ( $\lesssim 10^{7}$ años), estos sistemas albergan NSs jóvenes, con campos magnéticos más altos que sus pares en LMXBs $\left(B \sim 10^{11}-10^{12} \mathrm{G}\right)$. Si bien hasta el presente, en ninguno de estos sistemas se han detectado jets, en el Capítulo 2 de la Parte II exploraremos esa posibilidad, motivados por el descubrimiento de una nueva clase de binarias de rayos $\mathrm{X}$, con fulguraciones supergigantes breves en rayos X ( $\mathrm{SFXTs}^{14}$ ) que podrían estar asociadas a fuentes de emisión de rayos $\gamma$, originada en jets relativistas.

Cuando el flujo de materia acretada en un sistema binario de rayos $\mathrm{X}$ es continuo, el sistema muestra una luminosidad de rayos $\mathrm{X}$ relativamente constante que se denomina persistente. Sin embargo, en muchos de estos sistemas la masa se transfiere de manera irregular, a través de episodios de actividad que tienen una duración típica de semanas a meses, denominados outbursts. En cambio, la mayor parte del tiempo transcurre en un estado de quietud o quiescence en el que la acreción está fuertemente reducida, aunque no cesa y su radiación en rayos X es un factor $\sim 100$ menor. La duración y recurrencia de estos episodios varía fuertemente entre distintas fuentes. Mientras que la mayoría está activa sólo por algunas semanas o meses, hay una subclase de sistemas que sufren períodos de acreción prolongada que dura años o décadas (Wijnands, 2004).

En HMXBs, donde la materia acretada proviene del viento de la estrella de gran masa, y no de un disco de acreción, la inhomogeneidad o grumosidad del viento puede dar lugar también a comportamientos transitorios (Sidoli, 2009). Además, en los casos que la estrella de gran masa es del tipo espectral Be, este comportamiento puede surgir por la variabilidad intrínseca de la estrella primaria, o cuando el sistema tiene una órbita muy excéntrica, el proceso de acreción puede estar

\footnotetext{
${ }^{14}$ Supergiant Fast X-ray Transients
} 
sujeto a los pasos por el periastro, evidenciados en la periodicidad del régimen activo. En el caso de LMXBs, los ciclos transitorios pueden ser explicados en términos de una inestabilidad del disco de acreción que oscila entre un estado frío y neutro y otro caliente y ionizado provocando un fuerte incremento en la tasa de acreción (King \& Ritter, 1998). En cambio, durante los períodos de quietud, el disco recupera la masa perdida, lo que permite repetir el ciclo nuevamente.

\section{Formación de jets en sistemas binarios}

Simulaciones magnetohidrodinámicas muestran que la producción de jets en objetos compactos está gobernada por la formación de una torre magnética que emerge desde su superficie. Esta torre magnética posibilita la aceleración y colimación de las partículas eyectadas hasta velocidades relativistas (Kato, 2007). Inestabilidades introducidas, por ejemplo, por la interacción con el viento de la estrella donante, generan ondas de choque dentro del jet. Luego, a través del mecanismo de Fermi, las partículas adquieren suficiente energía $(\sim 1 \mathrm{GeV})$ para enfriarse emitiendo radiación $\gamma$ observable.

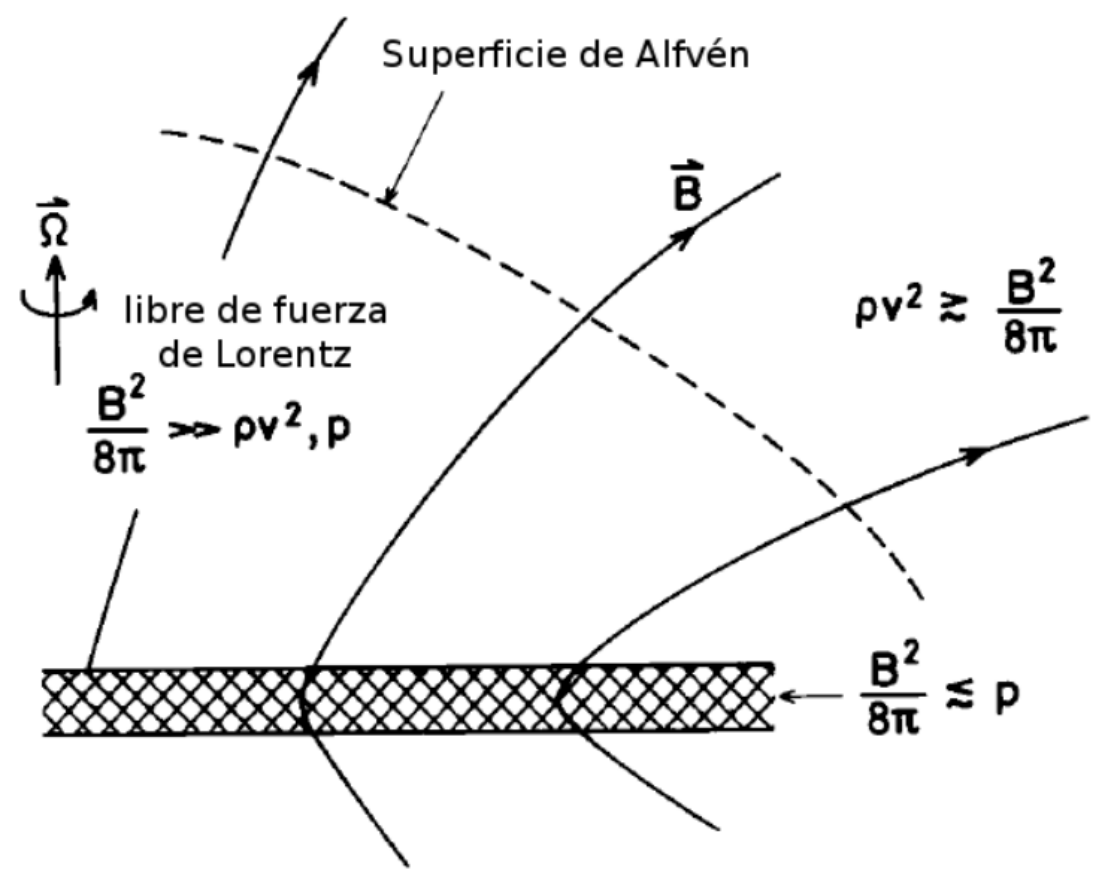

Figura 1.5: Esquema de las tres regiones que participan de la formación de un jet. La zona rayada representa un disco de acreción. El objeto compacto se ubica a la izquierda del diagrama (adaptada de Spruit, 2010).

En el modelo de aceleración magneto-centrífuga (Spruit, 2010) para la producción de jets se consideran tres regiones diferentes (ver Figura 1.5). La primera es el disco de acreción, donde la energía rotacional y la presión del fluido $p_{f}=\frac{1}{2} \rho v^{2}$ dominan sobre la energía magnética $p_{B}=$ $B^{2} / 8 \pi$. Como resultado, las líneas del campo magnético rotan con el disco, arrastradas por el 
fluido. La segunda es la región inmediatamente superior e inferior al disco, formada por una atmósfera tenue. Allí, la presión del campo magnético domina sobre la del gas y las partículas cargadas se mueven a lo largo de las líneas de campo, en lo que se denomina régimen libre de la fuerza de Lorentz: $(\boldsymbol{\nabla} \times \boldsymbol{B}) \times \boldsymbol{B}=0$.

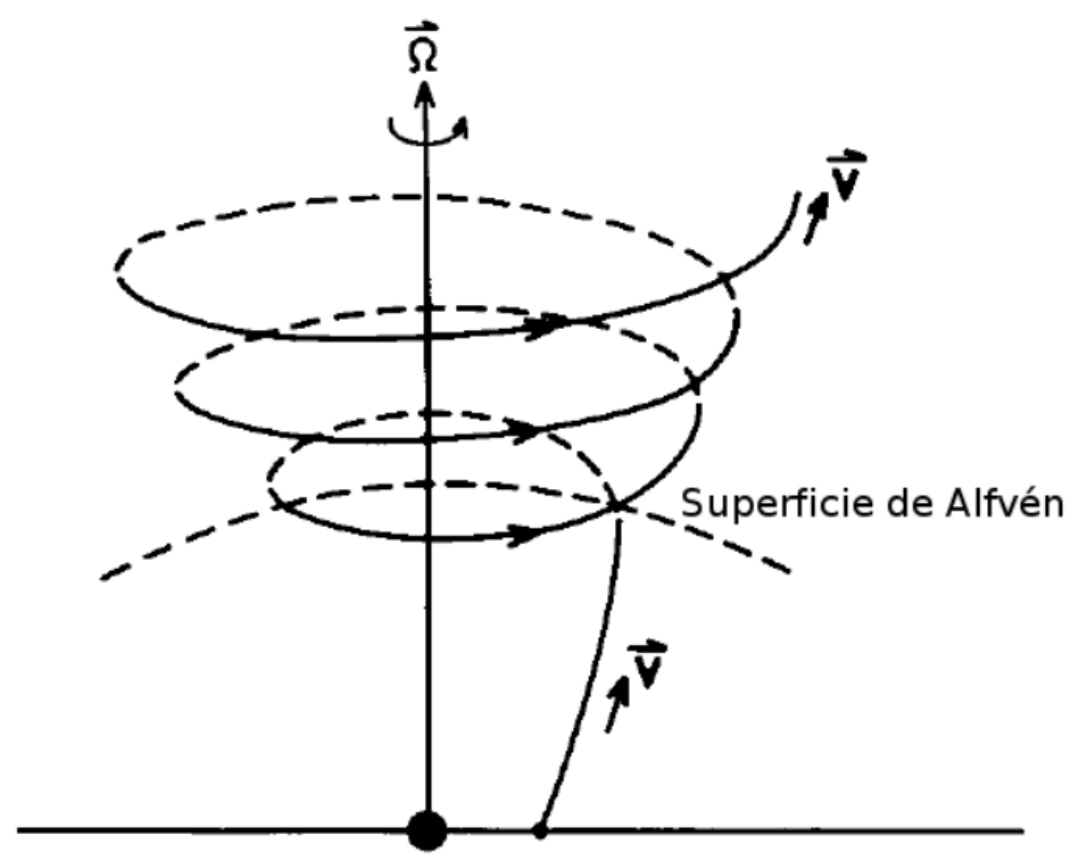

Figura 1.6: Esquema de la formación de una torre magnética (adaptada de Spruit, 2010).

Como el sistema se encuentra rotando, las partículas sienten una fuerza centrífuga que las acelera a lo largo de las líneas de fuerza en la dirección vertical (ver Figura 1.6). A medida que la partícula se aleja del objeto compacto, el campo magnético decrece hasta que su presión se iguala con la presión del gas $\left(p_{B}=p_{f}\right)$ en lo que se denomina superficie de Alfvén. A partir de esa superficie comienza la tercera región, donde las líneas de campo acompañan el movimiento espiral de las partículas, formando una torre magnética. Debido a que la densidad de energía magnética decrece desde la base de la torre hacia el exterior, las partículas son expulsadas del objeto compacto, en lo que se denomina jet. Si el campo magnético de la NS es muy intenso, la superficie de Alfvén se aleja de su superficie y las partículas del disco no pueden ser conectadas con la torre magnética, por lo que la formación del jet es inhibida.

La condición básica para la formación de jets, puede establecerse pidiendo que $R_{A} / R \leq 1$, donde $R_{A}$ es la distancia típica entre la superficie de Alfvén y el objeto compacto, de radio $R$. Expresando la tasa de acreción como $\dot{M}=4 \pi r^{2} \rho v(r)$, donde $v(r)=\sqrt{2 G M / r}$ es la velocidad de caída libre del fluido, y suponiendo que el campo magnético externo es dipolar, de manera tal 
que $B(r) / B(R)=(R / r)^{3}$, en $R_{A}$, se obtiene:

$$
\frac{B(R)^{2}}{8 \pi}\left(\frac{R}{R_{A}}\right)^{6}=\frac{\dot{M}}{8 \pi R_{A}^{2}} \sqrt{\frac{2 G M}{R_{A}}}
$$

Simplificando y despejando el radio de Alfvén, se encuentra:

$$
R_{A}=B(R)^{4 / 7} R^{12 / 7}(2 G M)^{-1 / 7} \dot{M}^{-2 / 7}
$$

de donde el cociente $R_{A} / R$ se reduce a:

$$
\frac{R_{A}}{R}=B(R)^{4 / 7} \dot{M}^{-2 / 7}\left(\frac{R^{5}}{2 G M}\right)^{-1 / 7}
$$

Considerando valores típicos para la masa $M \sim 1.4 \mathrm{M}_{\odot}$ y el radio $R \sim 10 \mathrm{~km}$, la condición básica se reduce a (Massi \& Kaufman Bernadó, 2008):

$$
R_{A} / R \simeq 0.87\left(\frac{B(R)}{10^{8} \mathrm{G}}\right)^{4 / 7}\left(\frac{\dot{M}}{10^{-8} \frac{\mathrm{M}_{\odot}}{\text { año }}}\right)^{-2 / 7} \leq 1
$$

De aquí se deduce que, para que una NS pueda formar jets, considerando una tasa de acreción promedio típica de $10^{-8} \mathrm{M}_{\odot}$ año ${ }^{-1}$, resulta necesario que su campo magnético superficial sea $B(R) \lesssim 10^{8} \mathrm{G}$.

\section{Erupciones termonucleares de rayos $\mathrm{X}$}

Uno de los fenómenos que atestiguan la presencia de una NS en un sistema binario de rayos X (descartando que se trate de un sistema binario que incluya a un agujero negro) son las erupciones termonucleares tipo-I (Type-I X-ray bursts, en inglés), que son destellos intensos de la emisión de rayos $\mathrm{X}$ que se produce como resultado de la quema termonuclear inestable en la superficie de NSs acretantes.

Observacionalmente, estas erupciones son caracterizadas por emisión térmica con un espectro tipo cuerpo negro con una temperatura máxima de $k T_{\mathrm{bb}} \sim 2-3 \mathrm{keV}$ y se distinguen por un incremento rápido de la emisión, $\lesssim 5 \mathrm{~s}$, seguido por un decaimiento exponencial más lento, de 10100 s de duración. Las propiedades observacionales como su duración, energía radiada o fluencia, y el tiempo de recurrencia de estas erupciones depende de las condiciones físicas a las que está sometida la capa de ignición del material, es decir, a su temperatura, espesor y a la abundancia química. Estas propiedades a su vez, dependen fuertemente de la tasa de acreción de materia (Bildsten, 1998; Fujimoto et al., 1981).

A medida que el $\mathrm{H}$ y/o He acretado se apila en la superficie de la NS, su temperatura y la densidad se incrementan, permitiendo la fusión de $\mathrm{H}$ en He de manera estable. A cierto punto, la temperatura y densidad en la base de la capa de material acretado (de algunos metros de espesor) resulta suficiente para iniciar la quema de He. Como este proceso es inestable, todo el $\mathrm{He}$ disponible en la capa se fusiona en $\mathrm{C}$ en una escala de tiempo de algunos segundos, produciendo la erupción. Una vez que la erupción finaliza, la acreción continúa y el proceso puede repetirse. 
En algunas fuentes estás erupciones son tan intensas que provocan la expansión de su fotósfera, en una erupción tipo $\mathrm{PRE}^{15}$. Se trata de erupciones particularmente brillantes en las que la luminosidad de la NS alcanza el límite de Eddington, lo que da como resultado que las capas superiores de la estrella se alejen momentáneamente de la superficie, producto de la presión de radiación ejercida desde la superficie. En los espectros, estas erupciones se caracterizan por presentar dos picos máximos en la evolución de su temperatura, que anti-correlacionan con la evolución del área de emisión. Cuando la fotósfera se expande, su área de emisión se incrementa y su temperatura disminuye, mientras que la luminosidad permanece aproximadamente constante, próxima al límite de Eddington. Cuando la erupción sobre su superficie termina, la presión de radiación disminuye y la fotósfera cede cayendo nuevamente hasta la superficie sólida. En ese momento, la temperatura se incrementa nuevamente por esa compresión, alcanzando un segundo pico coincidente con una superficie de emisión mínima. A partir de allí, la temperatura decae lentamente, a medida que la atmósfera radía un espectro térmico, tipo cuerpo negro, con un área de emisión relativamente constante, proporcional al cuadrado del radio de la estrella. La observación de estas erupciones de rayos $\mathrm{X}$ ha permitido así abrir una nueva oportunidad para el estudio detallado del comportamiento de la materia bajo condiciones de densidad y presión extremas, que se complementan mediante estudios de NSs aisladas y acretantes. En el Capítulo 5 de la Parte III de esta tesis, presentamos el estudio de la fase de enfriamiento de las 16 erupciones de rayos X detectadas en LMXB $4 \mathrm{U}$ 1820-30 y su consecuencias a la hora de determinar con gran precisión la masa y el radio de la NS albergada en ese sistema binario.

El hecho de que los picos de luminosidad alcanzados en las erupciones tipo PRE ocurran a la luminosidad de Eddington, abre la posibilidad de usar el flujo medido durante estas erupciones como candelas estándares para derivar la distancia a estas fuentes, en función de sus masas. Sin embargo, la fuerte dependencia con la desconocida composición del material de la fotósfera, introduce limitaciones en la determinación teórica de dicha luminosidad. Kuulkers et al. (2003) analizaron las erupciones PRE de 12 fuentes en cúmulos globulares con estimaciones independientes de la distancia, conocida por métodos fotométricos, encontrando que 8 de estas fuentes presentan luminosidades máximas en el valor crítico de $3.79 \pm 0.15 \times 10^{38} \mathrm{erg} \mathrm{s}^{-1}$, consistente con material pobre en H y una pequeña dispersión en las masas de las NSs en estas fuentes.

Por otra parte, unas pocas de estas fuentes han experimentado erupciones mucho mayores de incluso horas de duración, denominadas superbursts. Estos episodios extraordinariamente energéticos podrían evidenciar la quema inestable de $\mathrm{C}$ en estas fuentes (Strohmayer \& Bildsten, 2006).

La detección de pulsaciones durante las erupciones de tipo-I en $\sim 20$ de estos sistemas, del orden de los milisegundos, consistentes con los períodos de rotación de las NSs, así como la necesidad de contar con una superficie sólida sobre la que acumular el material acretado antes de la erupción, constituyen una prueba firme de la presencia de una NS en estos sistemas, descartando que se trate de agujeros negros.

\footnotetext{
${ }^{15}$ Del inglés, Photosperic Radius Expansion.
} 


\subsection{Modelos y configuraciones de estrellas de neutrones}

En esta sección presentamos las ecuaciones relativistas necesarias para construir modelos y configuraciones de estrellas esféricamente simétricas y en equilibrio hidrostático, usadas para representar la estructura de las NSs. Estas ecuaciones se complementan con una prescripción para las condiciones de la materia en su interior, que matemáticamente se traduce en una determinada ecuación de estado $\left(\operatorname{EoS}^{16}\right)$. Luego, en esta misma sección, estudiaremos apartamientos de la esfericidad, como producto de la rotación y de la presencia de campos magnéticos extremos que afectan a la estructura estelar. Finalmente daremos una serie de lineamientos que motivan el estudio de NSs en teorías alternativas a GR.

\subsubsection{Estrellas de neutrones en relatividad general}

La configuración más simple que puede proponerse para representar una NS surge de la suposición de que se trata de una estructura con simetría esférica y en equilibrio hidrostático.

Las ecuaciones de equilibrio hidrostático en GR se deducen a partir de las ecuaciones de campo de Einstein:

$$
G_{\mu \nu}=\frac{8 \pi G}{c^{4}} T_{\mu \nu}
$$

donde $G_{\mu \nu}$ es el tensor de Einstein que condensa la información de la curvatura del espacio-tiempo, mientras que $T_{\mu \nu}$ es el tensor de energía e impulso que para un fluido ideal, que puede escribirse como:

$$
T_{\mu \nu}=\left(\rho c^{2}+p\right) u_{\mu} u_{v}-p g_{\mu \nu}
$$

Aquí $\rho$ y $p$ son la densidad y la presión del fluido, $u$ la cuatrivelocidad y $g_{\mu \nu}$ es el tensor métrico. Las condiciones de estaticidad y simetría esférica simplifican esta métrica que en coordenadas esféricas, $r, \theta$ y $\phi$, se expresa de la siguiente manera:

$$
\mathrm{d} s^{2}=g_{\mu \nu} \mathrm{d} x^{\mu} \mathrm{d} x^{\nu}=e^{2 v(r)} \mathrm{d} t^{2}-e^{2 \lambda(r)} \mathrm{d} r^{2}-r^{2}\left(\mathrm{~d} \theta^{2}+\operatorname{sen}^{2} \theta \mathrm{d} \phi^{2}\right)
$$

donde $v(r)$ y $\lambda(r)$ son funciones a determinar a partir de la resolución de las ecuaciones de Einstein y describen completamente a la solución interior y exterior del espacio tiempo.

El tensor de Einstein, $G_{\mu \nu}$, se expresa como función del tensor de Ricci:

$$
G_{\mu \nu}=R_{\mu \nu}-\frac{1}{2} g_{\mu \nu} R
$$

donde $R=g^{\mu \nu} R_{\mu \nu}$ es el escalar de curvatura. Y, a su vez, el tensor de Ricci, $R_{\mu \nu}$, es una combinación de las conexiones, $\Gamma_{\mu \nu}^{\beta}=\frac{1}{2} g^{\alpha \beta} g_{\alpha \mu, \nu}+g_{\alpha v, \mu}+g_{\mu \nu, \alpha}$ :

$$
R_{\mu \nu}=\Gamma_{\mu \alpha, v}^{\alpha}-\Gamma_{\mu \nu, \alpha}^{\alpha}-\Gamma_{\mu \nu}^{\alpha} \Gamma_{\alpha \beta}^{\beta}+\Gamma_{\mu \beta}^{\alpha} \Gamma_{\nu \alpha}^{\beta} .
$$

\footnotetext{
${ }^{16}$ Del inglés, Equation of State.
} 
Luego, de las ecuaciones 1.10 y 1.12 sigue que los elementos del tensor de Ricci, $R_{\mu \nu}$, no nulos son:

$$
\begin{aligned}
& R_{00}=\left(-v^{\prime \prime}+\lambda^{\prime} v^{\prime}-\left(v^{\prime}\right)^{2}-\frac{2 v^{\prime}}{r}\right) e^{2(v-\lambda)} \\
& R_{11}=v^{\prime \prime}-\lambda^{\prime} v^{\prime}+\left(v^{\prime}\right)^{2}-\frac{2 \lambda^{\prime}}{r} \\
& R_{22}=\left(1+r v^{\prime}-r \lambda^{\prime}\right) e^{-2 \lambda}-1 \\
& R_{33}=R_{22} \operatorname{sen}^{2} \theta
\end{aligned}
$$

y, por lo tanto, su contracción resulta:

$$
R=\left(-2 v^{\prime \prime}+2 \lambda^{\prime} v^{\prime}-2\left(v^{\prime}\right)^{2}-\frac{2}{r^{2}}+\frac{4 \lambda^{\prime}}{r}-\frac{4 v^{\prime}}{r}\right) e^{-2 \lambda}+\frac{2}{r^{2}}
$$

Finalmente, sustituyendo estas ecuaciones en la Ecuación 1.11, se obtiene el siguiente sistema de ecuaciones diferenciales dado por:

$$
\begin{aligned}
\frac{8 \pi G}{c^{2}} \rho(r) & =\left(\frac{1}{r^{2}}-\frac{2 \lambda^{\prime}}{r}\right) e^{-2 \lambda}-\frac{1}{r^{2}} \\
-\frac{8 \pi G}{c^{4}} p(r) & =\left(\frac{1}{r^{2}}+\frac{2 v^{\prime}}{r}\right) e^{-2 \lambda}-\frac{1}{r^{2}} \\
-\frac{8 \pi G}{c^{4}} p(r) & =\left(v^{\prime \prime}+\left(v^{\prime}\right)^{2}-\lambda^{\prime} v^{\prime}+\frac{v^{\prime}-\lambda^{\prime}}{r}\right) e^{-2 \lambda}
\end{aligned}
$$

El teorema de Birkhoff establece la relación entre el elemento espacial de la diagonal de la métrica, con la masa encerrada, $m(r)$, como en la métrica de Schwarzschild:

$$
e^{-2 \lambda(r)}=1-\frac{2 G m(r)}{c^{2} r}
$$

y, por otra parte, usando la identidad de Bianchi, esto es $\nabla_{v} T^{\mu v}=0$, las cantidades $v, v^{\prime}$ y $v^{\prime \prime}$ pueden escribirse en función de $p, p^{\prime}$ y $p^{\prime \prime}$ para así arribar al siguiente conjunto de ecuaciones diferenciales (Oppenheimer \& Volkoff, 1939; Tolman, 1939).

\section{Ecuaciones de Tolman, Oppenheimer y Volkoff (TOV)}

Las ecuaciones de Einstein en el caso de una métrica estática con simetría esférica se reducen al siguiente sistema de ecuaciones diferenciales acopladas, conocidas como las ecuaciones de Tolman-Oppenheimer-Volkoff (TOV):

$$
\begin{aligned}
& \frac{d m(r)}{d r}=4 \pi r^{2} \rho(r) \\
& \frac{d p(r)}{d r}=-\frac{G \rho(r) m(r)}{r^{2}}\left[1+\frac{p(r)}{\rho(r) c^{2}}\right]\left[1+\frac{4 \pi r^{3} p(r)}{m(r) c^{2}}\right]\left[1-\frac{2 G m(r)}{c^{2} r}\right]^{-1}
\end{aligned}
$$


La Ecuación 1.22 describe la ley de continuidad de la masa, pero considerando la densidad total de energía en forma relativista. La Ecuación 1.23 representa el equilibrio hidrostático entre la atracción gravitatoria y la presión de la materia. Esta última, así escrita, presenta tres factores que corrigen a la expresión newtoniana para el equilibrio hidrostático. El primero y el segundo factor presentan correcciones provenientes de la relatividad especial y son de orden $v^{2} / c^{2}$, por lo que en el límite no relativista tienden a la unidad. El último factor incorpora una corrección de GR y su peso queda determinado por el término $G M / c^{2} R$. En el caso de las estrellas enanas blancas, donde $M \sim 1.4 \mathrm{M}_{\odot}$ y $R \sim 10^{4} \mathrm{~km}$, este término es del orden de $10^{-4}$, por lo que resulta suficiente utilizar la aproximación newtoniana para calcular su estructura. En cambio, para las dimensiones típicas de una NS, este factor resulta $G M / c^{2} R \sim 0.1$, por lo que los efectos relativistas no pueden ser despreciados.

El sistema de ecuaciones diferenciales acopladas dado por la Ecuación 1.22 y la 1.23 puede resolverse si se conoce la relación entre la presión y la densidad $p=p(\rho)$, o en forma equivalente, entre la presión y la densidad total de energía $p=p(\epsilon)$, la EoS. Así como en las enanas blancas la EoS está dominada por la presión originada en la degeneración del gas de electrones, en las NSs la presión es aportada, fundamentalmente, por un gas de neutrones degenerado. En general, la EoS es también función de la temperatura, sin embargo, los efectos térmicos pueden ser despreciados en la construcción de modelos de estrellas compactas, como veremos en la siguiente Subsección.

Para construir entonces una primera configuración, se procede proponiendo un valor para la densidad central $\rho_{\mathrm{c}}=\rho(r=0)$ y se integra el sistema de ecuaciones diferenciales acopladas desde $r=0$ hasta el borde $r=R$. Este borde exterior, en principio desconocido, queda determinado por la condición $p(R)=0$. Así, al alcanzar el borde, la masa total queda definida por $M=m(R)$, lo que caracteriza completamente a una configuración a través del par $(M, R)$. Repitiendo el proceso para otros valores de $\rho_{\mathrm{c}}$, se genera una familia de configuraciones acordes a la EoS elegida.

Bajo ciertas condiciones, la presión en el interior puede no ser capaz de soportar el peso de las capas externas de la estrella. De hecho, como se observa en la Ecuación 1.23, en GR, la presión es también una fuente del campo gravitatorio. Por lo tanto, su propio incremento, en vez de sustentar la estructura, puede terminar provocando el colapso gravitatorio. Esto significa que no todos los modelos obtenidos son necesariamente estables. El estudio de la estabilidad de los modelos es realizado a partir de un análisis de pequeñas oscilaciones en torno a las configuraciones de equilibrio. Existe un criterio de estabilidad simple que surge de una condición necesaria: dada una familia de soluciones parametrizada por la densidad central $\rho_{c}$, serán inestables aquellas que cumplan $d M / d \rho_{c}<0$ (Glendenning, 2000). Esta condición implica que la familia de soluciones estables deberá contener extremos para aquellos modelos que cumplan con $d M / d \rho_{\mathrm{c}}=0$. Los extremos, que dependen directamente de la EoS propuesta, son las llamadas: masa máxima $M_{\text {máx }} \sim 0.7-2.3 \mathrm{M}_{\odot}$ y masa mínima $M_{\text {mín }} \sim 0.1 \mathrm{M}_{\odot}$ (Haensel, 2003). Este resultado constituye la forma más directa de hacer una contrastación entre los modelos teóricos de EoSs a alta densidad y las observaciones. 


\subsubsection{La ecuación de estado}

Si bien en las últimas décadas se han podido realizar grandes avances en la materia, no existen resultados determinantes ni definitivos acerca de la EoS de las NSs, que permanece siendo un misterio en la actualidad. Para NSs con masas $M>\mathrm{M}_{\odot}$, con edades mayores a unos pocos años, los efectos de la temperatura, el campo magnético y su rotación son débiles y con el fin de investigar sus configuraciones, a primera aproximación, pueden ser despreciados totalmente. Así, la estructura de las NSs y, en particular, la masa máxima que pueden alcanzar, $M_{\text {máx }}$, queda completamente determinada por la EoS (Haensel et al., 2007).

Para construir modelos de configuraciones de NSs, es necesario conocer la EoS que gobierna la materia desde densidades del orden de unos pocos $\mathrm{g} \mathrm{cm}^{-3}$ hasta $\sim 5 \times 10^{15} \mathrm{~g} \mathrm{~cm}^{-3}$. Como vimos en la sección anterior, a densidades $\gtrsim 10^{6} \mathrm{~g} \mathrm{~cm}^{-3}$, la materia está fuertemente degenerada, por lo que la presión puede describirse como una función de la densidad, únicamente, $p=p(\rho)$. Hasta la densidad nuclear, $\rho_{0}=2.7 \times 10^{14} \mathrm{~g} \mathrm{~cm}^{-3}$ la EoS está bastante bien determinada Chamel et al. (2008). Sin embargo, para $\rho>\rho_{0}$ las incertezas crecen rápidamente, lo cual se refleja directamente en el desconocimiento de la masa máxima, $M_{\text {máx }}$ de estas estrellas.

La parte externa, que se extiende hasta la densidad $\rho_{\mathrm{ND}}$, puede obtenerse a partir de datos experimentales de núcleos ricos en neutrones y fórmulas semiempíricas de materia nuclear, por lo cual es bastante bien conocida gracias a una serie de experimentos (Haensel \& Pichon, 1994). Por el contrario, en la corteza interna, las incertezas crecen debido a que las propiedades de los núcleos son difíciles de establecer en los laboratorios y su interacción con el gas de neutrones (no tenida en cuenta en este modelo sencillo) comienza a jugar un papel más protagónico. Los resultados obtenidos por Baym et al. (1971), utilizando este método, muestran por ejemplo que, a densidades $\rho \sim 8 \times 10^{6} \mathrm{gr}^{-3}$, la corteza está compuesta por iones de ${ }^{56} \mathrm{Fe}$, mientras que, a densidades $\rho \sim 4.3 \times 10^{11} \mathrm{gr} \mathrm{cm}^{-3}$, el elemento que compone la red es ${ }^{118} \mathrm{Kr}$. Estos cálculos fueron incorporados por Douchin \& Haensel (2001) para modelar la EoS estándar que se presenta aquí en las figuras $1.7 \mathrm{y} 1.8$.

Las mediciones recientes de la masa de dos pulsares cercanos a las $2 \mathrm{M}_{\odot}$, PSR J1614-2230 $\left(1.97 \pm 0.04 \mathrm{M}_{\odot}\right.$ Demorest et al., 2010) y PSR J0348+0432 (2.01 $\pm 0.04 \mathrm{M}_{\odot}$ Antoniadis et al., 2013) imponen restricciones muy fuertes a la EoS para $\rho>\rho_{0}$ brindando información acerca de las fuertes interacciones de la materia nuclear a alta densidad. Lamentablemente, si bien las masas de NSs en sistemas binarios pueden ser determinadas con mucha precisión, no ocurre lo mismo con su radio, cuya determinación depende de inferencias a partir del espectro de la radiación proveniente de su superficie, y está afectado también por la precisión con que pueda ser determinada la distancia.

La incorporación de los efectos térmicos sobre la EoS, modifica su composición química, dando lugar a una distribución de núcleos con diferentes números másicos y atómicos, centrada en ciertos valores promedio $\langle A\rangle$ y $\langle Z\rangle$. A temperaturas más bajas, esta distribución se vuelve más acotada hasta que a $T=0$ se recupera la aproximación original de un solo núcleo (Burrows \& Lattimer , 1984).

Para cuantificar estos efectos, se introduce el siguiente parámetro de impurezas $Q$ que, en 


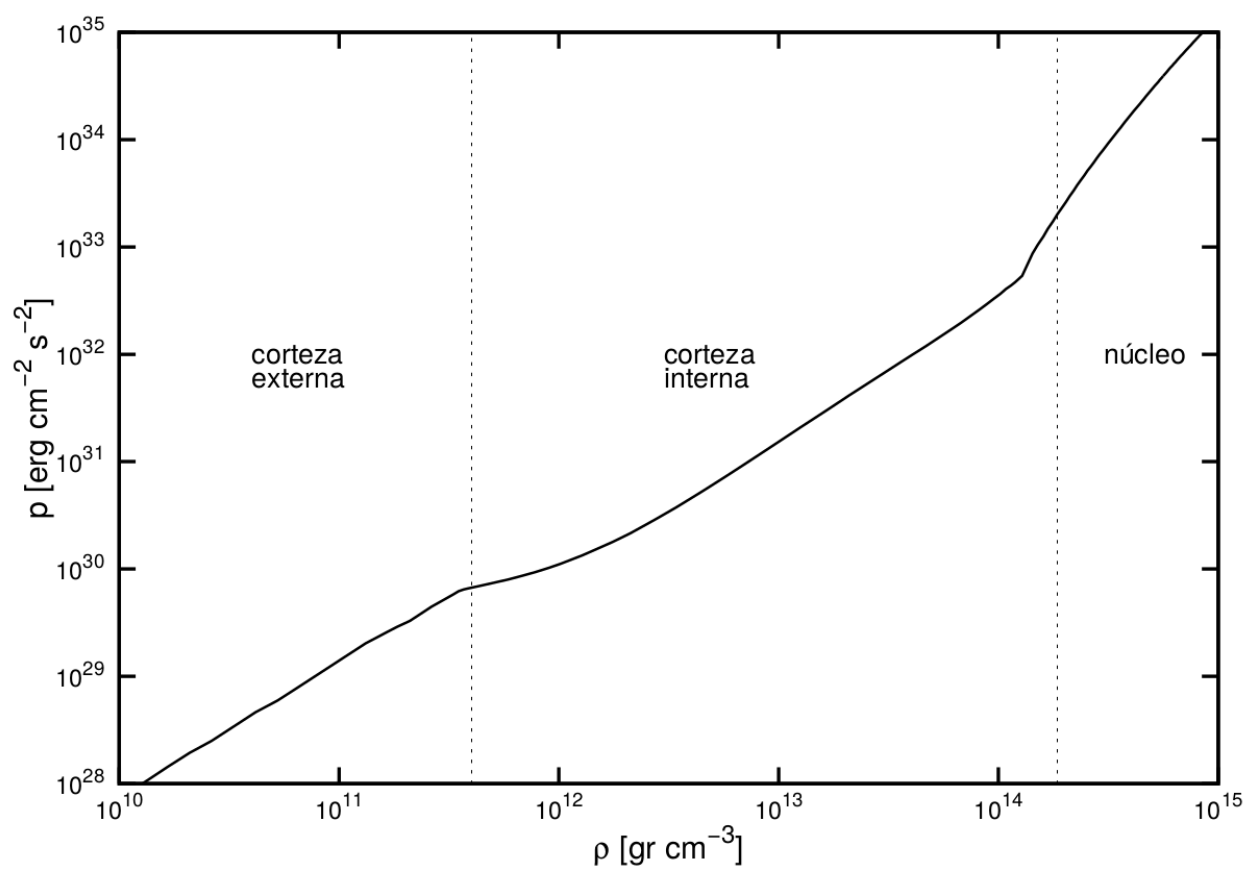

Figura 1.7: Ecuación de estado de Douchin \& Haensel (2001).
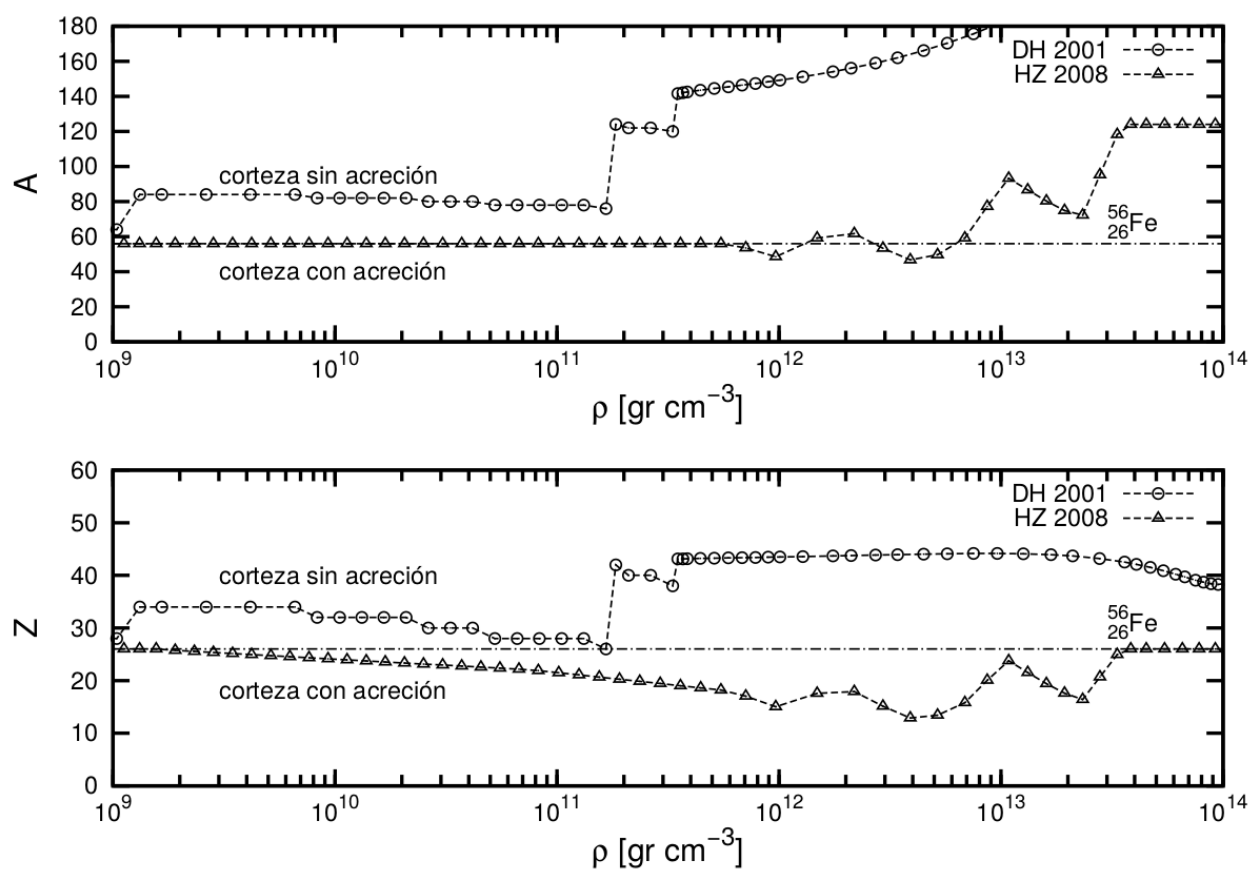

Figura 1.8: Comparación de la composición para dos EoSs. La ecuación Douchin \& Haensel (2001) y la ecuación de Haensel \& Zdunik (2008) que representa una corteza acretada. 
cierto modo, representa el "ancho" de esa distribución de núcleos:

$$
Q=\frac{1}{n} \sum_{\mathrm{imp}} n_{\mathrm{imp}}\left(Z-Z_{\mathrm{imp}}\right)^{2}
$$

donde $n$ es la densidad numérica de iones de carga $Z$ presentes en la red y $n_{\text {imp }}$ es la densidad numérica de impurezas de carga $Z_{\mathrm{imp}}$. De esta manera, $Q=0$ corresponde a una corteza pura, mientras que $Q>0$ representa la presencia de impurezas.

En la literatura se encuentra que la corteza de una NS aislada se corresponde con valores bajos de este parámetro como $Q \sim 0.001-0.01$ (Flowers \& Rutherman, 1977). En cambio, en cortezas sometidas a los efectos producidos por la acreción de materia, los cálculos realizados por Schatz et al. (1999), Brown (2000) y Jones (2004), presentan como resultado, valores de $1 \lesssim Q<100$. Este parámetro abarca varios órdenes de magnitud y presenta una gran incerteza en los modelos.

Una posible EoS correspondiente a una corteza afectada por un proceso de acreción fue calculada en detalle por Haensel \& Zdunik (2008). Como se observa en la Figura 1.8, la composición de una NS acretante resulta diferente de la correspondiente a materia catalizada a $T=0$. En general, a densidades bajas, los núcleos presentan $Z<20$, mientras que en la NS aislada, $Z=40-50$. A densidades medias, $A \sim 100$ mientras que para la materia catalizada, $A \sim 1000$. Finalmente, a las densidades más altas consideradas por los autores $\left(\rho \sim 10^{14} \mathrm{~g} \mathrm{~cm}^{-3}\right)$, las diferencias entre las EoSs desaparecen. Esto se debe a que a tales densidades, el comportamiento está fuertemente dominado por las propiedades del gas de neutrones.

\subsubsection{El diagrama Masa-Radio}

Uno de los objetivos principales que se persiguen a la hora de observar y modelar la emisión proveniente de la superficie de las NSs es conseguir medir sus masas y radios con precisión ya que estas dos propiedades globales macroscópicas funcionan como pruebas directas de la microfísica de su interior. La imposibilidad de determinar la EoS de la materia en el interior de las NSs a partir de primeros principios, torna a las observaciones de NSs fundamentales para imponer cotas a las diversas EoSs propuestas para las regiones ultra densas en el centro de estas estrellas, que puede servir de guía para el cálculo de la microfísica.

La EoS determina las propiedades macroscópicas de las NSs, particularmente, sus masas y radios posibles. Aceptando la unicidad de la EoS dada por la relación $(p-\rho)$, existe entonces una única familia de configuraciones posibles $(M-R)$, para una determinada teoría de gravedad. En la Figura 1.9 presentamos las familias de configuraciones $(M-R)$ para una serie de EoSs diferentes.

En principio, entonces, siendo capaces de determinar simultáneamente masas y radios de NSs a partir de observaciones, la relación $(M-R)$ podría ser invertida para deducir entonces la relación $(p-\rho)$. En la práctica, esto supone la medición precisa de masas en un rango de al menos un orden de magnitud, $\sim 0.1-2 \mathrm{M}_{\odot}$, aunque, en principio, NSs con masas menores al límite de Chandrasekhar para enanas blancas $\left(M \sim 1.4 \mathrm{M}_{\odot}\right)$ no son esperables en escenarios astrofísicos, lo que acota drásticamente este rango (Özel et al., 2012). Sin embargo, a pesar de estas limitaciones, la inversión del diagrama $(M-R)$ en al menos algunos puntos típicos, podría dar información 


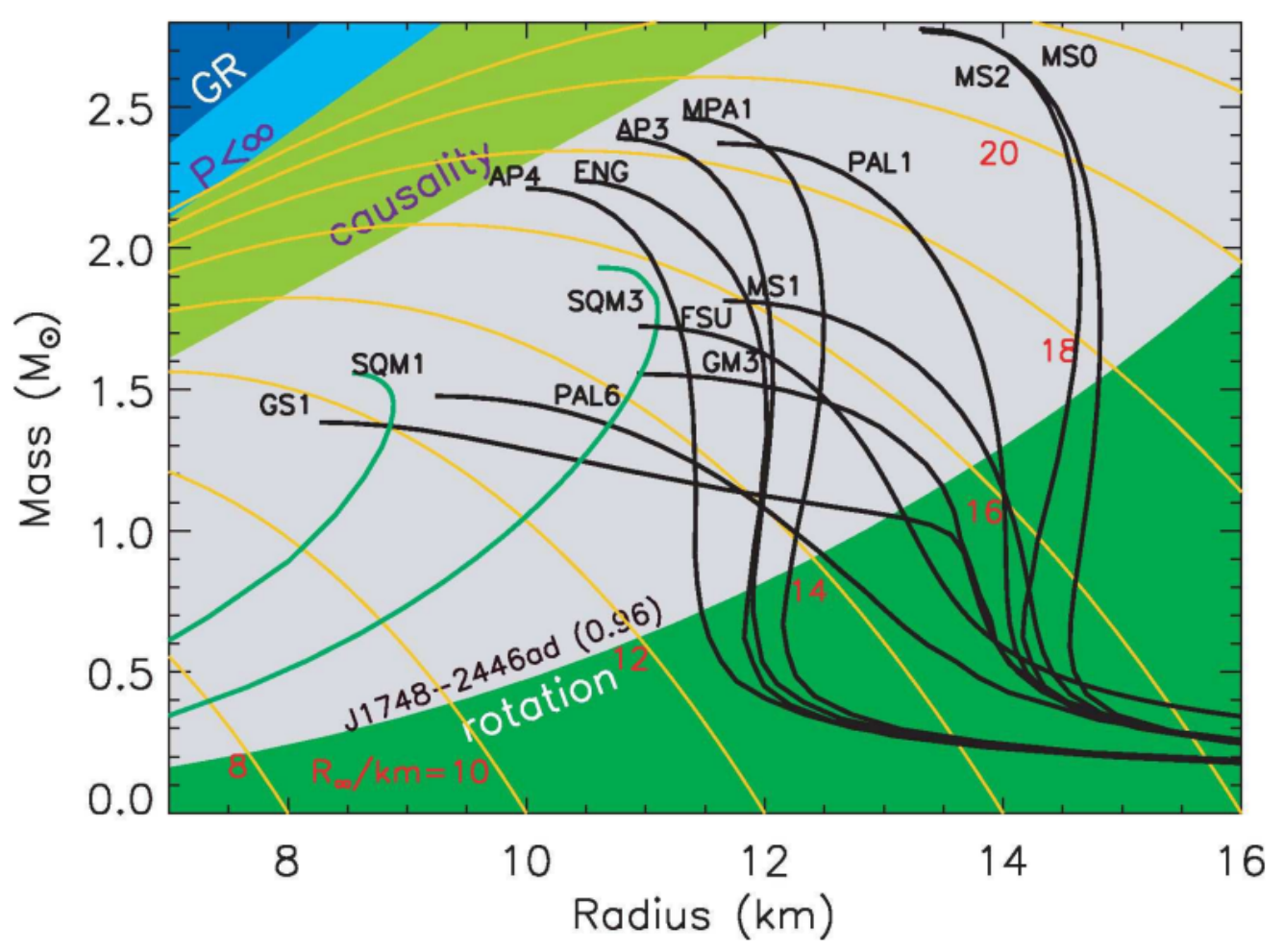

Figura 1.9: Diagrama masa-radio $(M-R)$ de Lattimer \& Prakash (2007) para las configuraciones de NSs obtenidas para EoSs típicas, representadas por curvas negras y estrellas de quarks, representadas por curvas verdes (SQM1 y SQM3). Las curvas ocre corresponden radios $R_{\infty}=R / \sqrt{1-2 G M / R c^{2}}$ constantes. La zona azul está excluida por el horizonte de GR $\left(R>2 G M / c^{2}\right)$, la zona celeste por la condición de presión finita $\left(R>(9 / 4) G M / c^{2}\right)$ y la región verde clara por causalidad $\left(R>3 G M / c^{2}\right)$. Por otro lado, la región verde oscura en la esquina inferior derecha representa regiones excluidas por las tensiones soportadas por la corteza del pulsar PSR J1748-2446ad, que rota a $716 \mathrm{~Hz}$ (Hessels et al., 2006).

importante del comportamiento de la materia por encima de la densidad de saturación nuclear (Steiner et al., 2013). En particular, la determinación de la masa máxima, $M_{\text {máx }}$ y en caso de ser posible, su radio asociado, resulta crucial.

Si bien la masa de las NSs puede determinarse con muchísima precisión a partir del análisis prolongado de sus órbitas en sistemas binarios, la medición de sus radios resulta mucho más dificultosa y está sujeta a una serie de indeterminaciones y problemas. Estos surgen naturalmente del hecho que una superficie asociada a un $R \sim 10 \mathrm{~km}$ a distancia del orden de los kpc, resulta imposible de resolver. Por lo tanto, se requiere introducir una serie de hipótesis sobre la físicas que dificultan su determinación directa. Más allá de las observaciones de NSs que giran con velocidades extremas, imponiendo límites superiores a sus tamaños (que da origen a la zona de exclusión de la zona inferior derecha de la Figura 1.9 de Hessels et al., 2006), algunos de los 
métodos ideados para la determinación de sus radios involucran: medidas espectrales, ya sea por el área de emisión asociada al espectro térmico de su superficie (Guillot et al., 2011; Heinke et al., 2006), la determinación del enrojecimiento de líneas de absorción o emisión asociadas a su atmósfera (Cottam et al., 2002), el enfriamiento posterior a erupciones termonucleares en su superficie (Güver et al., 2010); ajuste de los perfiles de emisión pulsada (Zavlin \& Pavlov, 1998). Si bien de estos análisis no puede concluirse una determinación precisa del radio, es interesante que se deduce una cota superior $\sim 12 \mathrm{~km}$ para todas estas estrellas.

Dadas las incertezas en la EoS a alta densidad, es interesante plantear cotas superiores a $M_{\text {máx }}$ basadas en argumentos físicos independientes. Los primeros cálculos de la masa máxima $M_{\text {máx }}$ en el marco de la GR se basaron en una EoS para un gas de neutrones que dio como resultado $M_{\text {máx }}=0.7 \mathrm{M}_{\odot}$, claramente descartado por la determinación reciente de dos pulsares con $M \sim 2 \mathrm{M}_{\odot}$. Así, esta condición restringe fuertemente el conjunto de EoSs aceptables para describir la naturaleza de la materia a $\rho \rho_{0}$. Por otra parte, cualquier EoS debe cumplir con la causalidad dada por la teoría especial de la relatividad, esto es, que la velocidad del sonido sea menor que la velocidad de la luz, es decir: $v_{s}=(\mathrm{d} P / \mathrm{d} \rho)^{1 / 2} \leq c$. Proponiendo una EoS límite que maximice esta condición, pueden construirse configuraciones de NSs con envolturas estándar a $\rho<\rho_{\mathrm{m}}$ y núcleo desconocido, pero causal, para $\rho>\rho_{\mathrm{m}}$. Para estrellas con masas entorno a las $2 \mathrm{M}_{\odot}$, la masa contenida en la capa externa es muy pequeña, de donde surge la siguiente desigualdad (Haensel et al., 2007):

$$
M_{\text {máx }} \leq M_{\text {máx }}^{\text {causal }}=3.0\left(\frac{5 \times 10^{14} \mathrm{~g} \mathrm{~cm}^{-3}}{\rho_{\mathrm{m}}}\right)^{\frac{1}{2}} \mathrm{M}_{\odot}
$$

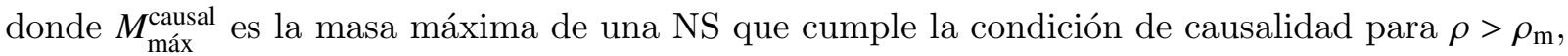
lo que lleva a una masa máxima de $\sim 4.1 \mathrm{M}_{\odot}$. Esta desigualdad es graficada como una región prohibida en la esquina superior izquierda del diagrama masa radio de la Figura 1.9.

\subsubsection{Configuraciones axisimétricas de estrellas de neutrones}

Se conocen una serie de mecanismos que pueden apartar de la simetría esférica una NS. La corteza de una NS es un arreglo de iones elástico que puede soportar por ejemplo, la presencia de abultamientos o montañas, como la corteza terrestre. El tamaño de la máxima distorsión que admitida por la estructura depende tanto de las propiedades mecánicas de la EoS como de la propia historia de la corteza que provoca la deformación (Haskell et al., 2006).

En algunos casos, los campos magnéticos en el interior de las NSs pueden ser tan intensos que resultan capaces de repercutir tanto sobre la EoS de la materia como sobre la propia estructura global de la estrella. Un análisis dimensional simple permite estimar cuál es la intensidad de campo magnético necesaria para afectar plenamente la estructura estelar. Igualando la energía magnética $\left.E_{\text {mag }} \sim 4 \pi R^{3} / 3\right)\left(B^{2} / 8 \pi\right)$ con la energía de ligadura gravitacional $E_{\text {grav }} \sim 3 G M^{2} / 5 R$, se obtiene el siguiente valor crítico:

$$
B_{\mathrm{cr}} \sim 1.4 \times 10^{18}\left(\frac{M}{1.4 \mathrm{M}_{\odot}}\right)\left(\frac{R}{10 \mathrm{~km}}\right)^{-2} \mathrm{G}
$$


valores relativamente menores de la intensidad del campo magnético también tendrán efectos sobre la estructura, aunque en menor medida (Lai \& Shapiro, 1991).

Cardall et al. (2001) estudiaron los límites del equilibrio hidrostático para configuraciones relativistas de NSs con campos magnéticos axisimétricos, incluyendo sus efectos sobre la EoS. Los autores mostraron que el campo magnético contribuye con una fuerza tipo centrífuga al tensor de energía e impulso total, produciendo apartamientos de la esfericidad hacia configuraciones elipsoidales. Además, en estos casos, la densidad central resulta menor que en el caso no magnético, admitiendo configuraciones de estrellas más masivas que en el caso esféricamente simétrico. Si los campos son demasiado intensos, la estabilidad de la estrella puede verse comprometida.

Los campos magnéticos actúan como la rotación, pero en sentido contrario. En el caso de la rotación, dada una cierta EoS, la masa máxima puede crecer por acumulación ecuatorial, disminuyendo a su vez la densidad central, pero dando lugar a una estrella ensanchada en el ecuador y achatada en los polos. En el caso de un campo magnético muy intenso, la densidad máxima puede incluso correrse del centro geométrico de la estrella, dando como resultado un distribución toroidal en la densidad. Esto se debe al efecto introducido por el campo magnético cuya tensión domina sobre la presión de la materia a densidades del orden de la densidad de saturación nuclear. En el caso de campos desordenados, podría darse lugar a campos magnéticos localmente incluso más intensos que $B_{\mathrm{cr}}$, pero el efecto global sobre la estructura se diluiría, dando como resultado una configuración prácticamente esférica.

Las condiciones necesarias para que las componentes poloidal y toroidal de un campo magnético axisimétrico adquieran una configuración estable fueron estudiadas por Braithwaite (2009) y Akgün et al. (2013). Si bien las configuraciones de NSs con campos magnéticos netamente poloidales o toroidales son inestables, estos autores encontraron soluciones en las que una combinación de ambas geometrías da lugar a configuraciones hidromagnéticamente estables. En el caso de NSs, el equilibrio es alcanzado si la componente toroidal domina sobre la poloidal, siempre que la energía total almacenada en el campo magnético sea $E_{\text {mag }} \lesssim 0.5 \times 10^{-4} E_{\text {grav }}$. En estas estrellas, la energía de ligadura gravitatoria es del orden de $E_{\text {grav }} \sim 10^{54} \mathrm{erg}$, por lo que en cualquier caso los efectos producidos por el campo magnético sobre la estructura estelar serán perturbativos Reisenegger (2013).

Cutler (2002) mostró que un campo magnético intenso predominantemente toroidal tiende naturalmente a distorsionar la estructura de una NS hacia una forma alargada en la dirección del eje magnético y perpendicular a su ecuador. Para campos magnéticos suficientemente intensos $\left(B \gg 10^{12} \mathrm{G}\right.$ ) el efecto introducido por el campo magnético domina por sobre la tendencia al ensanchamiento de la estrella producto de su rotación, permitiendo que sean alcanzadas elipticidades del orden de $\epsilon \lesssim 10^{-6}$.

Para rotadores rápidos como las NSs en LMXBs $(v \gtrsim 300 \mathrm{~Hz})$, con campos magnéticos ya evolucionados y, por lo tanto, débiles $\left(B \sim 10^{8} \mathrm{G}\right)$, se espera que su forma sea predominantemente ensanchada en el ecuador. Por el contrario, en el caso de NSs jóvenes y altamente magnetizadas $\left(B \sim 10^{14-15} \mathrm{G}\right)$ con bajas velocidades de rotación $(v \sim 0.1 \mathrm{~Hz})$, como los magnetares, se espera que su configuración de equilibrio bajo estas condiciones resulte en un alargamiento en la dirección perpendicular a su ecuador. La combinación de ambos efectos es sintetizada en la Figura 1 de 
Frieben \& Rezzolla (2012).

En el caso de los magnetares, como el campo magnético puede decaer significativamente en escalas de tiempo del orden de $10^{5}$ años, se espera que la configuración original alargada se vuelva inestable a partir de cierto punto, generando fuertes tensiones en su corteza. En el Capítulo 3 de la Parte II de esta tesis, exploraremos esto en detalle, motivados por la detección del "anti-glitch" en un magnetar AXP 1E 2259+586 (Archibald et al., 2013).

\subsubsection{Estrellas de neutrones en teorías alternativas de gravedad}

Desde 1917, cuando los grupos liderados por A. Eddington realizaron una expedición para verificar las predicciones de GR durante un eclipse solar, esta teoría ha sido sometida a una serie de pruebas que ha superado exitosamente, como las observaciones precisas de sistemas binarios de NSs. Sin embargo, todas estas pruebas corresponden a predicciones en el límite de campo gravitacional débil, mientras que, en el límite opuesto, de campo gravitacional fuerte, el progreso en las pruebas de la teoría ha sido mucho menor. Esto se debe esencialmente a que la fenomenología asociada a este límite es compleja y muchas veces explosiva, lo que dificulta encontrar observables dependientes únicamente del campo gravitacional. Es en este sentido que el estudio de NSs en teorías alternativas a GR juega un papel fundamental.

Las pruebas de GR en el régimen de campo fuerte usando NSs requieren de observatorios avanzados capaces de dar cuenta de los fenómenos asociados a estos objetos, que estarán disponibles en el futuro. Los dos parámetros utilizados para cuantificar el régimen del campo gravitatorio son su intensidad, $\phi=G M / r c^{2}$, y su curvatura, $\xi=G M / r^{3} c^{2}$. El campo gravitacional en la superficie de una NS puede medirse de manera directa a partir del corrimiento al rojo gravitacional, $z$, de líneas espectrales originadas en su atmósfera, $z=(1-2 \phi)^{-1 / 2}-1$. Al mismo tiempo, la curvatura está relacionada con la escala dinámica, $\tau=2 \pi / c \xi^{-1 / 2}$. Para su determinación serán necesarios observatorios con gran capacidad espectroscópica en escalas temporales de los milisegundos, que permitirán desentrañar los fenómenos asociados a los campos gravitacionales más intensos, como los existentes entorno a NSs.

\section{Teorías de gravedad alternativa $f(R)$}

El paradigma estándar para explicar la aceleración de la expansión del Universo se basa en la postulación de la existencia de una forma difusa de energía oscura que es descripta por una EoS correspondiente a un fluido exótico con densidad de energía negativa $(p \propto-\rho)$ que constituye aproximadamente el $70 \%$ de la densidad de energía crítica. Si bien el candidato natural para este fluido oscuro lo constituye la constante cosmológica, $\Lambda$, el valor aparentemente pequeño que se infiere de las observaciones cosmológicas se contradice con el esperado para la densidad de energía del vacío que se infiere de la física de partículas (Berti et al., 2015), lo que constituye uno de los principales problemas de la física teórica.

Como alternativa al modelo estándar de materia oscura fría y constante cosmológica se han propuesto una serie de alternativas que involucran la modificación de GR de manera de poder dar cuenta de las observaciones cosmológicas sin la necesidad de incorporar un fluido oscuro. En este 
contexto, las llamadas teorías $f(R)$ constituyen una de esas posibles alternativas. Inicialmente, las teorías $f(R)$ se tornaron atractivas por su capacidad de describir la aceleración cosmológica del Universo sin la necesidad de realizar una ajuste fino de la constante cosmológica.

Las teorías $f(R)$ constituyen una forma particular de teorías alternativas de gravedad. Estas teorías se construyen de manera que en el límite de campo gravitacional débil condicen con GR y, por tanto, son capaces de pasar naturalmente las pruebas de esta teoría, por ejemplo, en el Sistema Solar. Por el contrario, en el límite de campo gravitacional fuerte, ambas teorías divergen, siendo, por ejemplo la teoría de gravedad $f(R)$ cuadrática, $f(R)=R+\alpha R^{2}$, capaz de explicar las observaciones cosmológicas sin necesidad de acudir a la presencia de materia extraña como un fluido repulsivo con densidad de energía negativa (Capozziello \& Faraoni, 2011).

\section{Teorías $f(R)$ en el formalismo métrico}

En el formalismo métrico, las teorías $f(R)$ se construyen partiendo de la modificación del lagrangiano de Einstein-Hilbert, que se consigue agregando funciones del escalar de curvatura de Ricci, $R$, o introduciendo otros escalares de curvatura de mayor orden que aseguran la covarianza de la teoría. La acción de Einstein-Hilbert modificada se escribe entonces:

$$
S=\frac{c^{4}}{16 \pi G} \int \mathrm{d}^{4} x \sqrt{-g} f(R)+S_{\mathrm{m}}
$$

donde $S_{\mathrm{m}}$ denota los campos de materia y $f(R)$ es una función del escalar de curvatura $R$. En este campo, suele utilizarse la notación simplificada $f_{R}=f^{\prime}(R)$ y $f_{R R}=f^{\prime \prime}(R)$. Los modelos $f(R)$ deben ser funciones monótonas y convexas para evitar la existencia de taquiones que dan lugar a inestabilidades, y deben recuperar GR cuando la curvatura tiende a cero, es decir $f(R) \rightarrow R$ cuando $R \rightarrow 0$ (Capozziello \& Faraoni, 2011).

Así, las nuevas ecuaciones de campo en estas teorías se obtienen variando la acción, como en GR:

$$
f_{R}(R) R_{\mu v}-\frac{1}{2} f(R) g_{\mu v}-\left[\nabla_{\mu} \nabla_{v}-g_{\mu v} \square\right] f_{R}(R)=\frac{8 \pi G}{c^{4}} T_{\mu v}
$$

donde $\square=\frac{1}{\sqrt{-g}} \frac{\partial}{\partial x^{\nu}}\left(\sqrt{-g} g^{\mu \nu} \frac{\partial}{\partial x^{\mu}}\right)$ es el operador de d'Alembert. Estas ecuaciones de campo son más complejas e involucran derivadas de mayor orden en la métrica. En el límite en que $f(R)=R$, las ecuaciones de Einstein son recuperadas.

Es importante notar aquí que en las teorías $f(R)$ el escalar de curvatura, $R$, es una variable dinámica por lo que resulta necesaria una ecuación adicional para determinarlo. Tomando la traza de las ecuaciones de campo (1.28), se obtiene:

$$
3 \square f_{R}(R)+f_{R}(R) R-2 f(R)=-\frac{8 \pi G}{c^{4}} T
$$

donde $T$ es la traza del tensor de energía e impulso, $T_{\mu \nu}$. Cuando $f(R)=R$, esta ecuación se reduce a su análogo en GR: $R=-\frac{8 \pi G}{c^{4}}$. 
Para calcular NSs en estas teorías, una vez elegida la forma funcional $f(R)$, se procede con en GR. Se parte de proponer una métrica estática con simetría esférica y un tensor de energía e impulso para un fluido ideal y se deducen sistemas de ecuaciones diferenciales equivalentes a las de la sección anterior, que comúnmente se denominan ecuaciones TOV modificadas.

\section{Teorías $f(R)$ en el formalismo de Palatini}

Las teorías $f(R)$ en el formalismo métrico traen aparejada la existencia de campos dinámicos que ingresan a las ecuaciones de campo a través de derivadas de mayor orden que las de GR. En cambio, existe la posibilidad de realizar modificaciones a esta teoría clásica sin la necesidad de involucrar nuevos grados de libertad. Específicamente, es posible construir una teoría que modifique esencialmente el lado derecho de las ecuaciones de Einstein, esto es, agregar un tensor simétrico de segundo rango que se acople al tensor de energía e impulso. Es necesario que este término adicional tenga divergencia nula para mantener la validez del principio de equivalencia débil.

Una alternativa posible en esta dirección la constituyen las teorías $f(R)$ bajo el formalismo de Palatini. En este caso, la acción de Einstein-Hilbert se escribe de manera aparentemente similar a la misma $f(R)$ en el formalismo métrico (Capozziello \& Faraoni, 2011):

$$
S\left[g_{\mu \nu}, \Gamma, \psi_{\mathrm{m}}\right]=\frac{c^{4}}{16 \pi G} \int \mathrm{d}^{4} x \sqrt{-g} f(R)+S_{\mathrm{m}}\left[g_{\mu \nu}, \psi_{\mathrm{m}}\right]
$$

Sin embargo, la acción de la materia $S_{\mathrm{m}}$ depende en los campos de materia $\psi_{\mathrm{m}}$ y la métrica $g_{\mu \nu}$, y no de la conexión afín, que queda determinada dinámicamente.

Variando entonces la acción con respecto a la métrica y a la conexión (que es independiente), las ecuaciones de campo resultan:

$$
\begin{aligned}
f_{R}(R) R_{\mu \nu}(\Gamma)-\frac{1}{2} f(R) g_{\mu \nu} & =\frac{8 \pi G}{c^{4}} T_{\mu \nu} \\
\nabla_{\rho}\left[\sqrt{-g}\left(\delta_{\lambda}^{\rho} f_{R} g^{\mu \nu}-\frac{1}{2} \delta_{\lambda}^{\mu} f R g^{\rho \nu}-\frac{1}{2} \delta_{\lambda}^{v} f_{R} g^{\mu \rho}\right)\right] & =0
\end{aligned}
$$

donde $f_{R} \equiv \mathrm{d} f / \mathrm{d} R$ y $T_{\mu \nu}$ es el tensor de energía y momento, que satisface la ecuación de continuidad $\nabla_{\mu} T^{\mu \nu}=0$. Notar aquí que cuando $f(R)=R$, se recupera GR, ya que la Ecuación 1.32 se transforma en la definición de la conexión de Levi-Civita, al mismo tiempo que las ecuaciones de campo (1.31) resultan iguales a las de Einstein. En este marco, el hecho que $\Gamma_{\mu \nu}^{\sigma}$ es la conexión de Levi-Civita de la métrica, $g_{\mu \nu}$, emerge dinámicamente sin ser impuesto a priori por la acción, como en el caso de la teoría clásica de Einstein-Hilbert.

Además, tomando la traza de la Ecuación 7.2 se obtiene la siguiente ecuación algebraica para la curvatura $R$ :

$$
f_{R}(R) R-2 f(R)=\frac{8 \pi G}{c^{4}} T
$$


que se reduce a la traza estándar $R=-\frac{8 \pi G}{c^{4}} T$ en el límite de GR. Dada una función $f(R)$, es posible despejar $R$ de la ecuación, e insertando la solución nuevamente en la Ecuación 1.31, se obtiene un conjunto de ecuaciones de campo que dependen sólo de la métrica, $g_{\mu \nu}$, y del tensor de energía e impulso, $T_{\mu \nu}$. Así, la teoría tiene los mismos grados de libertad que GR, es decir, que sólo propaga un gravitón sin masa, de spin 2. Esta es una diferencia importante con la gravedad $f(R)$ en el formalismo métrico, que propaga un campo escalar adicional.

Como en la mayoría de las teorías alternativas a la gravedad, la métrica de Kerr es una solución de las ecuaciones de campo, las desviaciones esperables en el marco de los agujeros negros, que se modelan con soluciones de vacío, resultan poco viables para poner a prueba a GR. En cambio, la estructura de las estrellas compactas depende del acoplamiento de la gravedad con la materia en regiones de campo gravitacional intenso. Esto transforma a las NSs en candidatos muy valiosos para probar las teorías de gravedad en el régimen de campo fuerte, siendo capaces de poner a prueba e incluso descartar teorías que pueden parecerse a GR en el vacío, pero diferir completamente en la descripción del acoplamiento con la materia (Berti et al., 2015).

En los Capítulos 6 y 7 de la Parte IV de esta tesis presentamos los aportes originales que realizamos al estudio de NSs en teorías de gravedad cuadrática $f(R)$, tanto desde el formalismo métrico como bajo el formalismo de Palatini.

\subsection{Modelos de evolución del campo magnético}

Las ecuaciones que gobiernan tanto el enfriamiento como la evolución del campo magnético de las NSs requieren de un conocimiento preciso de los coeficientes de transporte en el interior estelar. Presentamos primero las propiedades de transporte en la corteza para luego pasar a estudiar la evolución temporal, incorporando otros elementos de la microfísica a altas densidades involucrados en estos modelos.

\subsubsection{Propiedades de transporte de la corteza}

Las características de la conductividad eléctrica y de la conducción térmica en la corteza de las NSs juegan un papel fundamental en la evolución magnética y en el enfriamiento de estos objetos. Mientras que la conductividad eléctrica, $\sigma$, es la responsable de regular la evolución del campo magnético, la conductividad térmica, $\kappa$, es la responsable de la difusión del calor en el interior de las estrellas, interviniendo directamente en el proceso de enfriamiento asociado.

Para poder calcular dichas conductividades es necesario conocer las propiedades de transporte en la materia estelar densa donde los electrones están fuertemente degenerados, a su vez que los iones están parcial o completamente ionizados, ubicados en una red cristalina. En estas condiciones, los electrones resultan ser los portadores fundamentales de la carga y el calor, por lo que es necesario estudiar los procesos de dispersión asociados, para determinar así los coeficientes de transporte en todo el interior estelar.

Las conductividades eléctricas y térmicas de electrones degenerados han sido estudiadas por diversos autores desde hace unos 50 años. Los primeros cálculos se basaron en un método variacio- 
nal (Ziman, 1960) que fue desarrollado por Flowers \& Itho (1976). Sus resultados fueron aplicados a NSs por Urpin \& Yakovlev (1980), quienes desarrollaron expresiones analíticas aproximadas para los coeficientes. Más adelante, estas expresiones fueron mejoradas por Itoh \& Kohyama (1993) incorporando un factor nuclear de forma, lo que permitió dar cuenta del tamaño finito de los núcleos atómicos. Estos autores introdujeron también un factor de Debye-Waller que describe las vibraciones del cristal. Este factor resulta importante a temperaturas altas, cuando las vibraciones del estado fundamental del cristal son considerables. Sin embargo, estos resultados presentaban fuertes discontinuidades en las conductividad, evidenciando una cierta inconsistencia en la teoría. En un trabajo más reciente, Baiko et al. (1998) mostró que en el régimen sólido, los cálculos de los procesos colisionales entre electrones y fonones deben incorporar la presencia de muchos fonones, eliminando la aproximación de un solo fonón. De esta manera, al volver a calcular las conductividades, las discontinuidades son fuertemente reducidas.

\section{Conductividad térmica}

El tensor de conductividad térmica involucra los procesos de transporte relacionados con todos los portadores: electrones, neutrones, protones y fonones. La radiación no es importante en el interior de la NS, aunque sí en la atmósfera, por lo que los fotones son excluidos. En la corteza, el transporte resulta dominado por los electrones, mientras que en el núcleo también son importantes las contribuciones de los neutrones y los protones (Baiko et al., 2001; Gnedin \& Yakovlev, 1995).

Ante la presencia de un campo magnético, el tensor $\hat{\kappa}$ se vuelve anisotrópico. Como la componente en la dirección normal al campo, $\kappa^{\perp}$, se ve fuertemente reducida, el calor tiende a fluir paralelo a las líneas de campo magnético, con una conductividad, $\kappa^{\|}$. Se puede mostrar que:

$$
\frac{\kappa^{\|}}{\kappa^{\perp}}=1+\left(\omega_{B} \tau\right)^{2}
$$

donde $\tau$ es el tiempo de relajación asociado a los electrones (Urpin \& Yakovlev, 1980) y $\omega_{B} \tau$ es la frecuencia de giro del electrón en un campo magnético de intensidad $B$ :

$$
\omega_{B}=\frac{e B}{m_{e}^{*} c}
$$

Así, cuando el parámetro de magnetización es $\omega_{B} \tau \gg 1$, los efectos del campo magnético sobre el transporte resultan cruciales.

\section{Conductividad eléctrica}

La contribución que resulte dominante sobre la conductividad eléctrica total depende de si la materia se encuentra en estado líquido o sólido. El paso de una a otra fase está regulado por la temperatura de fusión, $T_{m}$, dada por:

$$
T_{m}=3.04 \times 10^{7}\left(\frac{Z}{26}\right)^{5 / 3}\left(\frac{170}{\Gamma}\right) \times \mathrm{K}
$$


donde $x=\left(Z \rho_{6} / A\right)^{1 / 3}, Z$ y $A$ son los números atómicos y másicos del ión, $\Gamma=Z^{2} e^{2} / a k_{b} T, a=$ $\left(3 / 4 \pi n_{\mathrm{i}}\right)^{1 / 3}, n_{\mathrm{i}}$ es la densidad numérica de iones y $\rho_{6}=\rho / 10^{6} \mathrm{gr} \mathrm{cm}^{-3}$. La conductividad eléctrica está determinada principalmente por la dispersión de los electrones debida a los iones cuando $T>T_{m}$ y por la dispersión de los electrones debida a los fonones, $f$, cuando $T<T_{m}$. Las siguientes son expresiones analíticas aproximadas de la conductividad para ambos procesos:

$$
\begin{gathered}
\sigma_{\text {iones }}=8.53 \times 10^{21} \frac{x^{3}}{Z \Lambda_{\text {iones }}\left(1+x^{2}\right)} \mathrm{s}^{-1} \\
\sigma_{\mathrm{f}}=1.21 \times 10^{28} \frac{\sqrt{u^{2}+0.017}}{T u} \frac{x^{4}}{2+x^{2}} \mathrm{~s}^{-1}
\end{gathered}
$$

donde $\Lambda_{\mathrm{p}}$ es el logaritmo de Coulomb de la red de iones, $u=0.45 T / T_{D}$ y $T_{D}$ es la temperatura de Debye:

$$
T_{D}=3.4 \times 10^{6}\left(\frac{Z}{A}\right)^{1 / 2} x^{3 / 2} \mathrm{~K}
$$

La contribución de la dispersión de electrones por impurezas se vuelve importante a altas densidades y bajas temperaturas:

$$
\sigma_{\text {imp }}=8.53 \times 10^{21} x \Lambda_{\text {imp }} \frac{Z}{Q} \mathrm{~s}^{-1}
$$

donde $\Lambda_{\text {imp }}$ es el logaritmo de Coulomb de las impurezas y $Q$ es el parámetro de impurezas.

Una vez calculados los valores para los coeficientes correspondientes a cada proceso de dispersión, la conductividad total resulta:

$$
\frac{1}{\sigma}=\frac{1}{\sigma_{\text {iones }}}+\frac{1}{\sigma_{\mathrm{f}}}+\frac{1}{\sigma_{\text {imp }}}
$$

Cuando el campo magnético es poco intenso $\left(B \lesssim 10^{12} \mathrm{G}\right)$, los tensores de conductividad eléctrica y térmica se vuelven isotrópicos. Esto significa que sus componentes paralelas y normales al campo resultan equivalentes. En este régimen, el cálculo de la difusión óhmica resulta más simple porque el problema contiene esta nueva simetría.

La acreción de elementos livianos sobre la corteza puede traer aparejada la ignición de reacciones nucleares en sus capas más externas. Por cada reacción nuclear se libera una energía $E \sim 1 \mathrm{MeV} /$ nucleón. Esta energía contribuye al aumento de la temperatura de la corteza y, por ende, a la disminución de su conductividad. Además, la acreción de materia incorpora impurezas a la corteza de la NS, lo que reduce considerablemente la conductividad eléctrica a baja temperatura y alta densidad dentro de la corteza.

\subsubsection{Evolución del campo magnético}

En esta sección nos abocamos a introducir algunas propiedades del campo magnético de la NSs, analizando aspectos de su configuración interna y la geometría ligada a la corteza de la estrella para luego presentar las ecuaciones que gobiernan su evolución. 


\section{Campo magnético en la corteza}

Mientras que la estructura a gran escala del campo magnético externo de una NS está muy bien representada por un dipolo, la distribución real del campo magnético en su interior es poco conocida. Esto es producto de la imposibilidad de realizar mediciones directas, lo que supedita la contrastación de los modelos del campo magnético interior queda a la búsqueda de otros observables.

Las simulaciones numéricas muestran que los campos internos involucran tanto componentes poloidales como toroidales y que sus intensidades relativas dependen de la posición en el interior (Reisenegger, 2003). Cálculos bidimensionales muestran que, mientras que la distribución inicial del campo magnético evoluciona drásticamente en las primeras etapas $\left(t<10^{5}\right.$ años), a grandes escalas de tiempo, el campo puede adquirir una configuración más estable, predominantemente dipolar (Pons \& Geppert, 2007).

Todo campo magnético con simetría axial puede expresarse como la suma de dos componentes ortogonales, una poloidal y otra toroidal (Raedler, 2000):

$$
\boldsymbol{B}=\boldsymbol{B}_{\mathrm{pol}}+\boldsymbol{B}_{\text {tor }}
$$

Ambas componentes pueden ser derivadas a partir de dos funciones potenciales escalares $\tilde{S}$ y $\tilde{T}$ :

$$
\begin{aligned}
& \boldsymbol{B}_{\mathrm{pol}}=-\boldsymbol{\nabla} \times(\boldsymbol{r} \times \boldsymbol{\nabla} \tilde{S}) \\
& \boldsymbol{B}_{\mathrm{tor}}=-\boldsymbol{r} \times \boldsymbol{\nabla} \tilde{T}
\end{aligned}
$$

donde $\boldsymbol{r}$ es el vector radial.

En coordenadas esféricas, $r$ y $\theta$, la parte angular de las funciones escalares puede desarrollarse en polinomios de Legendre, como sigue:

$$
\begin{aligned}
& \tilde{S}(r, \theta)=C \sum_{l} \frac{P_{l}(\cos \theta)}{r} s_{l}(r, t) \\
& \tilde{T}(r, \theta)=C \sum_{l} \frac{P_{l}(\cos \theta)}{r} T_{l}(r, t)
\end{aligned}
$$

donde $P_{l}(\cos \theta)$ es el polinomio de Legendre de orden $l$ y $C$ es la constante de normalización adecuada. En particular, un campo puramente dipolar corresponde a un único modo poloidal, $l=1$, en ausencia de componentes toroidales. Es decir, que su función potencial asociada resulta:

$$
\tilde{S}(r, \theta)=\frac{\cos \theta}{r} s(r, t)
$$

A esta función particular $s(r, t)$ se la conoce como la función de Stokes. 


\section{Ecuación de inducción}

Para un fluido de gran conductividad eléctrica que se desplaza a velocidades no relativistas, que constituyen las hipótesis de la magnetohidrodinámica, los campos electromagnéticos obedecen las ecuaciones de Maxwell en la siguiente forma:

$$
\begin{aligned}
\boldsymbol{\nabla} \times \boldsymbol{E} & =-\frac{1}{c} \frac{\partial \boldsymbol{B}}{\partial t} \\
\boldsymbol{\nabla} \cdot \boldsymbol{B} & =0 \\
\boldsymbol{\nabla} \times \boldsymbol{B} & =\frac{4 \pi}{c} \boldsymbol{j}
\end{aligned}
$$

con la correspondiente ecuación constitutiva asociada:

$$
\boldsymbol{j}=\sigma\left(\boldsymbol{E}+\frac{\boldsymbol{u}}{c} \times \boldsymbol{B}+\boldsymbol{E}^{(e)}\right)
$$

donde $\boldsymbol{E}$ es el campo eléctrico, $\boldsymbol{B}$ es el campo magnético, $\boldsymbol{j}$ es la densidad de corriente y $\boldsymbol{u}$ es la velocidad del fluido. Además, $\sigma$ es la conductividad eléctrica, que caracteriza al fluido en cuestión. Por último, $\boldsymbol{E}^{(e)}$ representa la fuerza electromotriz externa a la que está sometido el sistema que en el caso en que estamos interesados aquí no juega ningún rol.

Las ecuaciones de Maxwell en el régimen de la magnetohidrodinámica pueden ser reducidas a una ecuación diferencial que involucre sólo al campo magnético $\boldsymbol{B}$. Para ello, podemos despejar $\boldsymbol{E}$ de la Ecuación 1.51 y reemplazarlo en la Ecuación 1.48, y luego sustituir $\boldsymbol{j}$ de acuerdo con la Ecuación 1.50 para entonces obtener:

$$
\frac{\partial \boldsymbol{B}}{\partial t}=-\frac{c^{2}}{4 \pi} \boldsymbol{\nabla} \times\left(\frac{1}{\sigma} \boldsymbol{\nabla} \times \boldsymbol{B}\right)+\boldsymbol{\nabla} \times(\boldsymbol{u} \times \boldsymbol{B})
$$

En la literatura, a esta relación se la conoce como la ecuación de inducción. Si el campo magnético es conocido para un determinado instante de tiempo, la evolución del campo magnético se halla resolviendo la ecuación de inducción con condiciones de contorno adecuadas. Una vez encontrado $\boldsymbol{B}$, el campo $\boldsymbol{E}$ y la corriente $\boldsymbol{j}$ pueden ser calculados directamente.

En esta ecuación puede observarse que la evolución del campo magnético depende de dos términos. El primero representa su difusión o disipación óhmica mientras que el segundo representa el transporte global del campo por efecto del movimiento del fluido que arrastra consigo las líneas de campo magnético. A éste último se lo denomina término convectivo.

Cuando los campos magnéticos involucrados no son muy intensos $\left(B \lesssim 10^{12} \mathrm{G}\right)$, los tensores de conductividad se vuelven isotrópicos, pudiendo ser representados por cantidades escalares. En este caso, despreciando los términos de mayor orden que el dipolar en el desarrollo del campo magnético (Ecuación 1.46), la ecuación vectorial de inducción, a través de la función de Stokes, puede llevarse a una ecuación diferencial unidimensional, simplificando enormemente los cálculos.

El rotor de un campo vectorial $\boldsymbol{F}$ en coordenadas esféricas es: 


$$
\begin{aligned}
\boldsymbol{\nabla} \times \boldsymbol{F}= & \frac{1}{r \operatorname{sen} \theta}\left(\frac{\partial}{\partial \theta}\left(\operatorname{sen} \theta F_{\phi}\right)-\frac{\partial F_{\theta}}{\partial \phi}\right) \check{\boldsymbol{r}}+ \\
& +\left(\frac{1}{r \operatorname{sen} \theta} \frac{\partial F_{r}}{\partial \phi}-\frac{1}{r} \frac{\partial}{\partial r}\left(r F_{\phi}\right)\right) \check{\boldsymbol{\theta}}+\frac{1}{r}\left(\frac{\partial}{\partial r}\left(r F_{\theta}\right)-\frac{\partial F_{r}}{\partial \theta}\right) \check{\boldsymbol{\phi}}
\end{aligned}
$$

Dado entonces un campo dipolar descripto por la función potencial escalar de Stokes (según la Ecuación 1.47). Reemplazando su expresión en la Ecuación 1.43 y calculando entonces los rotores con la fórmula 1.53, las componentes del campo magnético en coordenadas esféricas resultan:

$$
\begin{aligned}
B_{r} & =\frac{2 \cos \theta}{r^{2}} s(r, t) \\
B_{\theta} & =-\frac{\operatorname{sen} \theta}{r} \frac{\partial s}{\partial r} \\
B_{\phi} & =0
\end{aligned}
$$

A partir de estas definiciones, y considerando que el campo magnético evoluciona según la ecuación de inducción (1.52), deduciremos la ecuación diferencial que debe cumplir la función $s(r, t)$. Para ello, comenzamos por calcular $\boldsymbol{\nabla} \times \boldsymbol{B}$, utilizando la Ecuación 1.53:

$$
\boldsymbol{\nabla} \times \boldsymbol{B}=\left(-\frac{\operatorname{sen} \theta}{r} \frac{\partial^{2} s}{\partial r^{2}}+\frac{2 \operatorname{sen} \theta}{r^{3}} s\right) \check{\boldsymbol{\phi}}
$$

Aplicando nuevamente la Ecuación 1.53, obtenemos:

$$
\boldsymbol{\nabla} \times\left(\frac{1}{\sigma} \boldsymbol{\nabla} \times \boldsymbol{B}\right)=\left(-\frac{2 \cos \theta}{r^{2} \sigma} \frac{\partial^{2} s}{\partial r^{2}}+\frac{4 s \cos \theta}{r^{4} \sigma}\right) \check{\boldsymbol{r}}-\frac{\operatorname{sen} \theta}{r} \frac{\partial}{\partial r}\left(r\left(-\frac{1}{r \sigma} \frac{\partial^{2} s}{\partial r^{2}}+\frac{2 s}{r^{3} \sigma}\right)\right) \check{\boldsymbol{\theta}}
$$

Considerando un flujo de materia con simetría esférica, caracterizado por una velocidad $\boldsymbol{u}=u_{r} \check{\boldsymbol{r}}$, el producto vectorial $\boldsymbol{u} \times \boldsymbol{B}$ resulta no nulo únicamente en la dirección del versor $\check{\boldsymbol{\phi}}$ :

$$
\boldsymbol{u} \times \boldsymbol{B}=u_{r} B_{\theta} \check{\boldsymbol{\phi}}=u_{r} \frac{\operatorname{sen} \theta}{r} \frac{\partial s}{\partial r} \check{\boldsymbol{\phi}}
$$

Luego, aplicando la fórmula 1.53, obtenemos:

$$
\boldsymbol{\nabla} \times(\boldsymbol{u} \times \boldsymbol{B})=\frac{2 \cos \theta}{r^{2}} u_{r} \frac{\partial s}{\partial r} \check{\boldsymbol{r}}-\frac{\operatorname{sen} \theta}{r} \frac{\partial}{\partial r}\left(u_{r} \frac{\partial s}{\partial r}\right) \check{\boldsymbol{\theta}}
$$

Por otro lado, la derivada temporal del campo magnético, dada por la Ecuación 1.54, resulta:

$$
\frac{\partial \boldsymbol{B}}{\partial t}=\frac{2 \cos \theta}{r^{2}} \frac{\partial s}{\partial t} \check{\boldsymbol{r}}-\frac{\operatorname{sen} \theta}{r} \frac{\partial}{\partial r}\left(\frac{\partial s}{\partial t}\right) \check{\boldsymbol{\theta}}
$$

Luego, reemplazando las ecuaciones 1.58 y 1.59 en la ecuación de inducción (1.52), para la componente radial $\check{\boldsymbol{r}}$ encontramos:

$$
\frac{2 \cos \theta}{r^{2}} \frac{\partial s}{\partial t}=\left(-\frac{2 \cos \theta}{r^{2} \sigma} \frac{\partial^{2} s}{\partial r^{2}}+\frac{2 \cos \theta}{r^{2}} \frac{2 s}{r^{2} \sigma}\right)\left(-\frac{c^{2}}{4 \pi}\right)-u_{r} \frac{\partial s}{\partial r} \frac{2 \cos \theta}{r^{2}}
$$


y para la componente angular no trivial $\check{\boldsymbol{\theta}}$ :

$$
-\frac{\operatorname{sen} \theta}{r} \frac{\partial}{\partial r}\left(\frac{\partial s}{\partial t}\right)=-\frac{\operatorname{sen} \theta}{r} \frac{\partial}{\partial r}\left(-\frac{1}{\sigma} \frac{\partial^{2} s}{\partial r^{2}}+\frac{2 s}{r^{2} \sigma}\right)\left(-\frac{c^{2}}{4 \pi}\right)+\frac{\operatorname{sen} \theta}{r} \frac{\partial}{\partial r}\left(u_{r} \frac{\partial s}{\partial r}\right)
$$

Simplificando ambos miembros se deduce que las dos igualdades se cumplen simultáneamente si la función $s=s(r, t)$ obedece la siguiente ecuación diferencial unidimensional en la coordenada radial $r$ :

$$
\frac{\partial s}{\partial t}=\frac{c^{2}}{4 \pi \sigma}\left(\frac{\partial^{2} s}{\partial r^{2}}-\frac{2 s}{r^{2}}\right)-u_{r} \frac{\partial s}{\partial r} .
$$

Así, dado un valor inicial para el campo magnético en la superficie de la NS, $B_{\mathrm{d}}(t=0)=B(r=$ $R, t=0)=s(r=R, t=0) \operatorname{sen} \theta / R$, el campo magnético a un instante posterior cualquiera es:

$$
B_{\mathrm{d}}(t)=s(r=R, t) \frac{\operatorname{sen} \theta}{R}=\frac{s(R, t)}{s(R, 0)} B_{\mathrm{d}}(t=0)
$$

Como la función escalar $s(r, t)$ está determinada a menos de una constante, podemos elegirla igual a la unidad en la superficie para el tiempo inicial. Luego, el campo magnético en la superficie al tiempo $t$, se obtiene mediante la siguiente ecuación:

$$
B_{\mathrm{d}}(t)=s(R, t) B_{\mathrm{d}}(0)
$$

Bajo estas condiciones, el problema de la evolución del campo magnético en la corteza de la NS se restringe a la resolución de la ecuación diferencial unidimensional (1.62) para la función potencial de Stokes. Para resolverla, es necesario dar condiciones iniciales y de contorno apropiadas al problema en cuestión.

\subsection{Las estrellas de neutrones desde los rayos $\mathrm{X}$}

Como hemos visto a lo largo de este capítulo, las NSs constituyen uno de los conjuntos de fuentes que predominan en el cielo de rayos X. Si bien su descubrimiento y una vasta cantidad de conocimiento de estas estrellas ha sido obtenido en ondas de radio, la banda de los rayos X es la región del espectro más apta para poder obtener información directa de su superficie, e inferir así las propiedades físicas de su interior. Sin embargo, para ello es menester comprender los escenarios astrofísicos en que se hallan inmersas y desarrollar técnicas observacionales adecuadas a la banda de los rayos X. Presentamos aquí los instrumentos de rayos $\mathrm{X}$ que recolectaron los datos que utilizamos en esta tesis y completamos este capítulo dando una breve introducción a las técnicas de análisis de datos de rayos X enfocada al estudio de las NSs.

Contrariamente a la mayoría de los instrumentos de mayores longitudes de onda, que miden un flujo integrado, los detectores de rayos $\mathrm{X}$ identifican cada fotón de manera individual. Esto se debe a que los fotones de rayos $\mathrm{X}$ tienen energías relativamente altas, por lo que su impacto resulta fácilmente detectable. A su vez, como los flujos son relativamente bajos, en general, los fotones pueden ser contados uno por uno. 
Los datos de los instrumentos de rayos $\mathrm{X}$ se almacenan en archivos de eventos que contienen información de la energía, tiempo de detección y posición de cada fotón que llega al detector. En estos archivos, se registra además el estado de la electrónica asociada a los detectores y la orientación del satélite que puede variar durante la exposición. Estos archivos de eventos se distribuyen en formato FITS $^{17}$ que es un formato binario y portable específicamente diseñado para datos astronómicos.

Estas listas de eventos asociadas a los fotones detectados constituyen un espacio multidimensional. Proyectando ese espacio en una o dos dimensiones, y agrupando los eventos según algún criterio, se pueden construir imágenes, curvas de luz o espectros de la fuente. La reducción de los datos y la extracción de los productos científicos finales específicos de cada instrumento, requiere de una serie de técnicas que describiremos más adelante. En esta tesis se utilizan también datos y resultados de otros instrumentos. En esos casos, se referencia a los trabajos originales.

\subsubsection{Observatorios de rayos $\mathrm{X}$}

Como los rayos X son fuertemente absorbidos por la atmósfera terrestre, para poder realizar estudios en esta banda del espectro electromagnético, es necesario colocar los instrumentos sensibles a estas energías en el espacio. A continuación se presentan las características principales de los observatorios de rayos $\mathrm{X}$ usados en esta tesis.

\section{Rossi X-ray Timing Explorer (RXTE)}

El explorador de rayos X de alta resolución temporal, Rossi X-ray Timing Explorer (RXTE) (Bradt et al., 1993), fue lanzado el 30 de diciembre de 1995 por la $N A S A$ hasta una órbita de $580 \mathrm{~km}$ de altura, que cubre en 96 min de período. Si bien fue diseñado para entre dos a cinco años de trabajo en el espacio, $R X T E$ estuvo en operaciones durante 16 años hasta ser decomisada el 5 de enero de 2012. Este satélite ha proveído a la comunidad de una cantidad de datos sin precedentes, relativos a agujeros negros, enanas blancas y NSs, cubriendo escalas temporales desde los microsegundos hasta años a lo largo de un amplio espectro en el rango de energía desde los 2 hasta los $250 \mathrm{keV}$, que incluso hoy en día siguen dando nuevos resultados a partir del análisis minucioso de su archivo.

$R X T E$ contaba con tres instrumentos científicos a bordo: un monitor de todo el cielo $\left(\mathrm{ASM}^{18}\right.$, Levine et al., 1996), un arreglo de contadores proporcionales (PCA ${ }^{19}$, Jahoda et al., 1996; Zhang et al., 1993) y el experimento temporal de rayos X de alta energía (HEXTE ${ }^{20}$, Gruber et al., 1996; Rothschild et al., 1998). La Figura 1.10 presenta un esquema del observatorio y sus tres instrumentos a bordo. El ASM observó > $80 \%$ del cielo durante cada órbita con una resolución de $3^{\prime} \times 15^{\prime}$, y consistía de tres cámaras con un campo de $6^{\circ} \times 90^{\circ}$ cada una y un área efectiva de $\sim 30 \mathrm{~cm}^{2}$, siendo sensible en el rango de $1.5-12 \mathrm{keV}$ con una resolución temporal de $0.125 \mathrm{~s}$. El ASM

\footnotetext{
${ }^{17}$ http://fits.gsfc.nasa.gov

${ }^{18}$ Del inglés, All-Sky Monitor

${ }^{19}$ Del inglés, Proportional Counter Array

${ }^{20}$ Del inglés, High-Energy X-ray Timing Experiment
} 


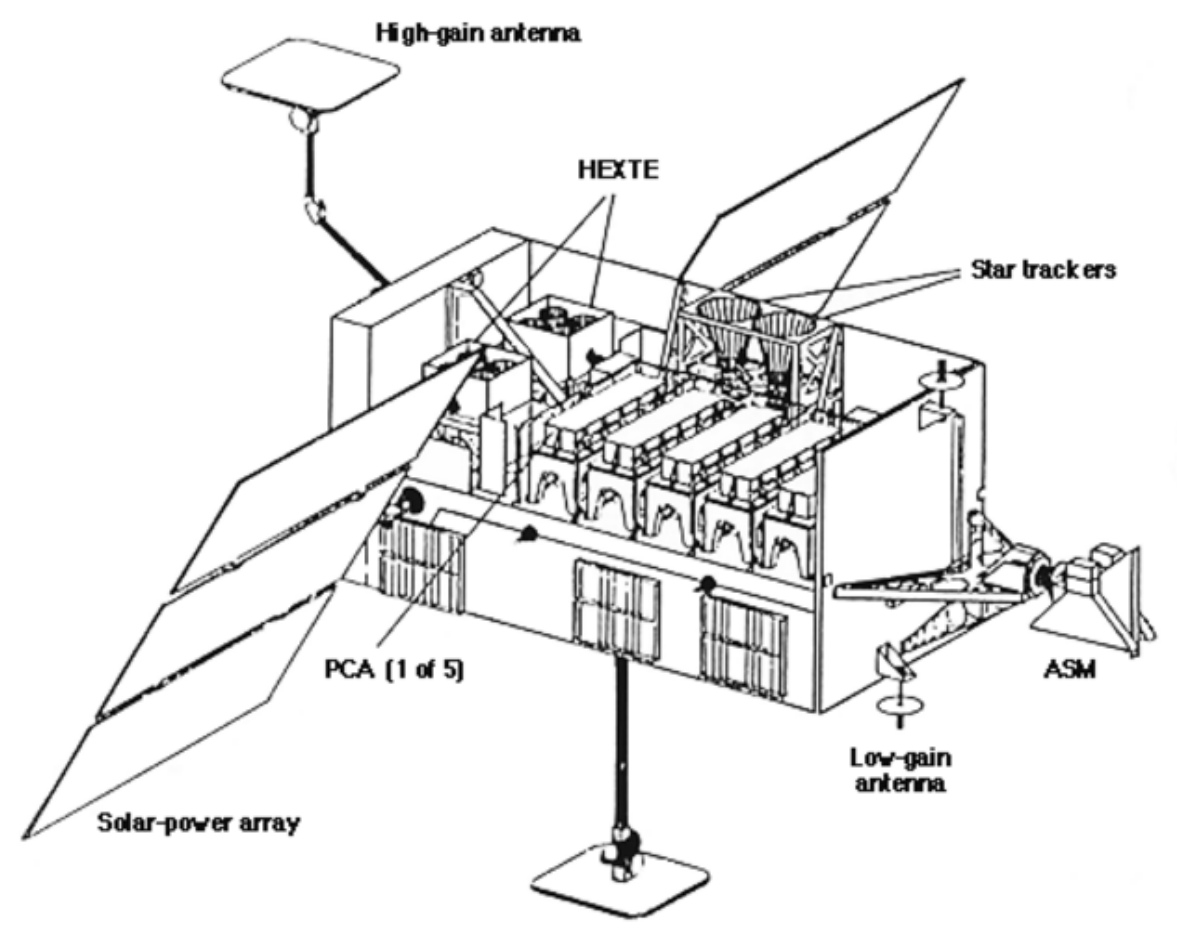

Figura 1.10: Una vista esquemática del satélite $R X T E$ y sus instrumentos a bordo, tomada de https://heasarc.gsfc.nasa.gov/Images/xte/xte_spacecraft.gif

se utilizaba para obtener curvas de luz de largo término de las fuentes de rayos $\mathrm{X}$ persistentes más brillantes así como para detectar nuevos fuentes transitorias o períodos de actividad de fuentes transitorias conocidas. El instrumento principal a bordo de $R X T E$ era PCA, que consistía de cinco contadores proporcionales sensibles en el rango de energía de 2-60 keV con un área efectiva total de $\sim 6500 \mathrm{~cm}^{2}$, una resolución temporal de $\sim 1 \mu$ s y una resolución en energía de $\sim 1 \mathrm{keV}$ a $6 \mathrm{keV}$.

La combinación de la gran área efectiva con y la alta resolución temporal con que fue dotado el instrumento PCA, hacen a RXTE un satélite único para el estudio de variabilidad temporal de fuentes de rayos X, que aprovecharemos en el Capítulo 5 de la Parte III de esta tesis donde estudiamos la evolución temporal de las propiedades espectrales de una NS que se enfría en un sistema binario en escalas de 10-20 s.

\section{X-ray Multi-Mirror Mission (XMM-Newton)}

La misión de rayos X de espejos múltiples, X-ray Multi-Mirror Mission (XMM-Newton), fue lanzada el 10 de diciembre de 1999 desde Kourou, en la Guyana Francesa y situado en una órbita de 48 horas de período (Jansen et al., 2001). El satélite posee dos tipos de telescopios diferentes: tres telescopios Wolter con una serie de detectores de rayos $\mathrm{X}$ en sus focos, $\mathrm{y}$ un 
telescopio óptico/UV de $30 \mathrm{~cm}$ de apertura con un detector CCD en su plano focal, por lo que permite realizar observaciones simultáneas en dos regiones del espectro electromagnético. $X M M$ Newton cuenta con tres instrumentos científicos a bordo, que son la cámara de imágenes de fotones europea $\left(\right.$ EPIC $\left.^{21}\right)$, el espectrómetro de reflexión $\left(\right.$ RGS $^{22}$, den Herder et al., 2001) y un monitor óptico ( $\mathrm{OM}^{23}$, Mason et al., 2001), indicados en el esquema de la Figura 1.11.

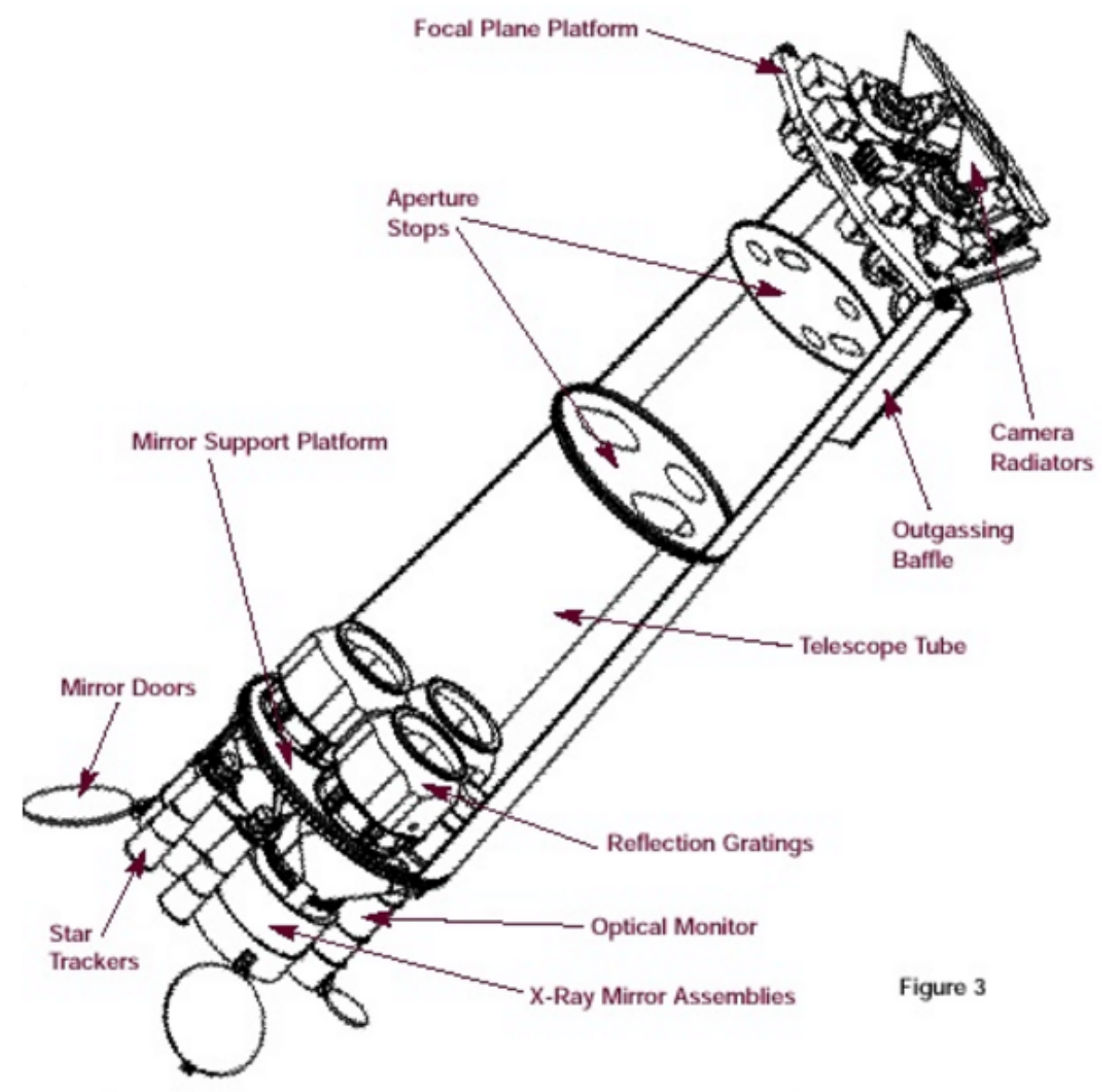

Figura 1.11: Una vista esquemática del satélite $X M M-N e w t o n$ y sus instrumentos a bordo, tomada de http://xmm.esac.esa.int/external/xmm_user_support/documentation/technical/ Spacecraft/. Los detectores descriptos se ubican sobre el plano focal.

La cámara EPIC está equipada con dos CCDs MOS (Turner et al., 2001) y uno PN (Strüder et al., 2001) que funcionan como espectrómetros de imagen. La cámara EPIC ofrece la posibilidad de realizar imágenes extremadamente sensibles en un campo de $30^{\prime}$ en el rango de energía de 0.15-12 keV con resolución espectral moderada $(E / \Delta E \sim 20-50)$ y resolución angular de $\gtrsim 6^{\prime \prime}$. La

\footnotetext{
${ }^{21}$ Del inglés, European Photon Imaging Camera.

${ }^{22}$ Del inglés, Reflection Grating Spectrometer.

${ }^{23}$ Del inglés, Optical Monitor.
} 
cámara EPIC pn, de características similares, puede ser operada con resolución temporal de hasta $0.03 \mathrm{~ms}$, y cuenta con un área efectiva de $1300 \mathrm{~cm}^{2}$. RGS está diseñado para obtener espectros de fuentes puntuales en el eje focal con muy alta resolución espectral $(E / \Delta E \sim 100-500)$ en el rango de energía de $0.33-2.5 \mathrm{keV}$ con un área efectiva de $\sim 140 \mathrm{~cm}^{2}$.

\section{Chandra X-ray Observatory (Chandra)}

El observatorio de rayos X Chandra (Weisskopf et al., 2000) fue lanzado el 23 de julio de 1999 y puesto en una órbita de 64 horas de período. Posee cuatro capas anidadas de espejos Wolter que da lugar a una resolución angular de $\sim 0.5^{\prime \prime}$, lo que lo convierte en el mejor telescopio de rayos X disponible hasta la actualidad. En el plano focal se encuentran dos detectores de rayos X: el espectrómetro de imágenes avanzado ( $\mathrm{ACIS}^{24}$, Garmire et al., 2003) y la cámara de alta resolución $\left(\mathrm{HRC}^{25}\right.$, Kenter et al., 2000). HRC provee un amplio campo de visión de $30^{\prime} \times 30^{\prime}$ con un área efectiva de $225 \mathrm{~cm}^{2}$ a $1 \mathrm{keV}$ y está diseñada para producir imágenes, con relativamente baja resolución espectral, en el rango de 0.1-10 keV. El detector ACIS es sensible en el rango de $0.3-10 \mathrm{keV}$ y cubre un campo de $16^{\prime} \times 16^{\prime}$ con un área efectiva de $\sim 340 \mathrm{~cm}^{2}$ y resolución espectral de $\sim 0.1 \mathrm{keV}$ a $1.5 \mathrm{keV}$ y baja resolución temporal, de $3.2 \mathrm{~s}$. Además, el telescopio permite interponer redes de difracción de baja y alta energía (LETG, 0.1-5 keV y HETG, 0.5$10 \mathrm{keV}$, respectivamente) para llevar a cabo espectroscopía de alta resolución $(\sim 3 \mathrm{eV}$ a $1 \mathrm{keV})$ que no fueron utilizadas en esta tesis.

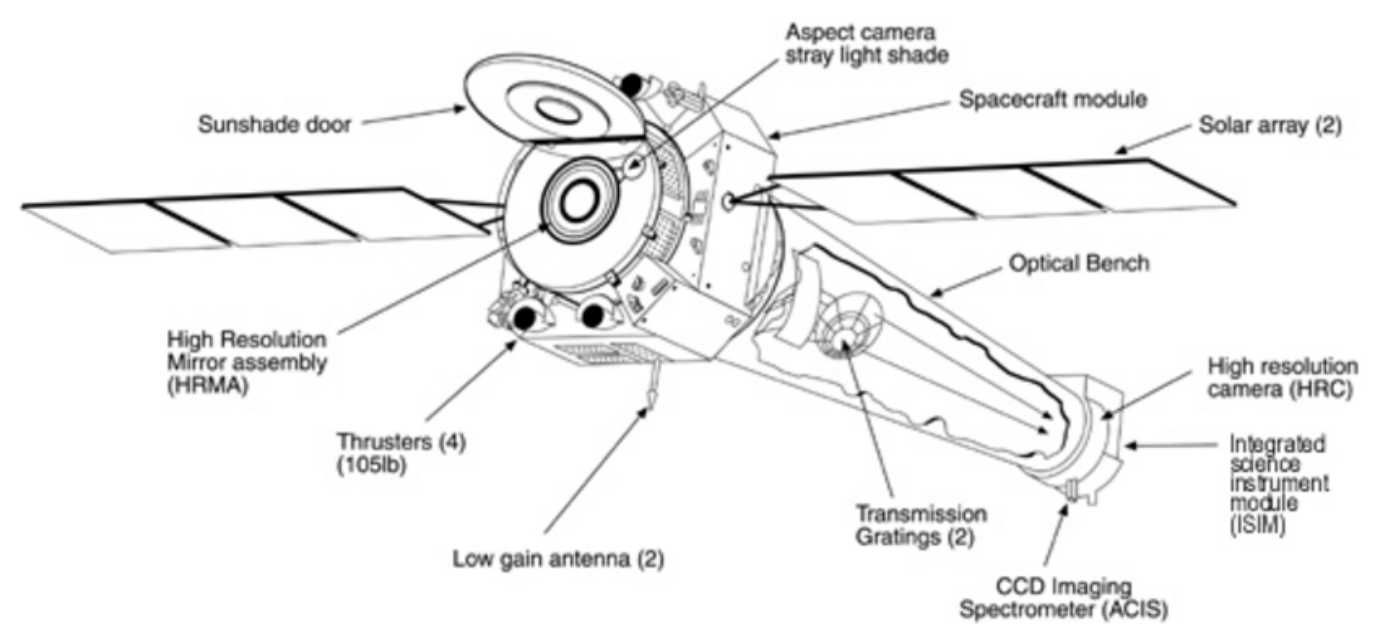

Figura 1.12: Una vista esquemática del satélite Chandra y su instrumental a bordo, tomada de http://chandra.harvard.edu/graphics/resources/illustrations/

En el Capítulo 4 de la Parte III haremos uso de observaciones de la cámara EPIC de $X M M$ Newton del SNR G290.1-0.8 para realizar un estudio detallado de la emisión extendida del rema-

\footnotetext{
${ }^{24}$ Del inglés, Advanced CCD Imaging Spectrometer.

${ }^{25}$ Del inglés, High Resolution Camera.
} 
nente y realizar una búsqueda de un objeto compacto en su interior. Para ello, incorporaremos imágenes de la cámara ACIS de Chandra con la resolución angular más alta disponible en la actualidad.

\subsubsection{Técnicas de análisis de datos de rayos $\mathrm{X}$}

Hasta el presente, la única fuente a partir de la cual podemos recabar información de las NSs es la radiación electromagnética que recibimos de ellas, ya sea originada en su propia superficie, o en su entorno más próximo. Como se mencionó anteriormente, las NSs fueron primeramente detectadas en la banda de radio. Luego, con el advenimiento de las nuevas generaciones de satélites, se realizaron nuevos avances producto del análisis de fotones de rayos $\mathrm{X}$ provenientes de ellas. Como la resolución angular con que cuentan los instrumentos de rayos X no permite resolver espacialmente a estos objetos, para poder determinar parámetros físicos asociados a sus propiedades y evolución, es necesario el desarrollo de técnicas de espectroscopía y análisis temporal que nos han permitido ampliar nuestro conocimiento de su naturaleza. Una revisión didáctica de todas estas técnicas puede encontrarse en Arnaud et al. (2011).

\section{Imágenes y análisis morfológico}

Las imágenes de rayos $\mathrm{X}$ se obtienen a partir de la proyección del espacio de eventos sobre las coordenadas del cielo, llamadas $\mathrm{X}$ e $\mathrm{Y}$ en los archivos. Como, por ejemplo, en XMM-Newton las coordenadas de los fotones son randomizadas en píxeles virtuales con resolución espacial mucho mayor que la del propio telescopio, los eventos deben ser agrupados para producir una imagen real. Las imágenes deben ser filtradas para rechazar los eventos que no correspondan a fotones, corregidas para evitar los píxeles dañados y normalizadas a través de mapas de exposición, similares a los flats de la astronomía óptica.

Como, además, los eventos tienen codificada la energía del fotón asociado (en la columna PI o PHA), las imágenes pueden filtrarse en diferentes bandas de energía que luego son combinadas usando filtros para producir imágenes de falso color, lo que permite analizar la morfología de fuentes extendidas. La lista de eventos disponible abre un abanico de posibilidades para poder realizar tratamientos estadísticos sobre las imágenes. Un ejemplo de ello lo constituyen los mapas específicos de energía media o mediana, que utilizaremos en el Capítulo 4 de la Parte III. Para producirlos, a cada píxel de la imagen final se le asigna un valor que resulta de la media o mediana de la energía de todos los fotones detectados en esa posición, lo que permite analizar variaciones espectrales de la fuente con alta resolución espacial.

\section{Curvas de luz}

Las curvas de luz o series temporales se construyen agrupando los eventos del archivo en función de la columna TIME, a partir del conteo de la cantidad de eventos detectados en cada bin temporal. En caso de considerar también la energía de cada fotón, mediante la columna PI o PHA, puede deducirse el flujo integrado por unidad de tiempo. Además, filtrando los eventos por 
diferentes bandas de energía, pueden producirse nuevas curvas de luz, lo que permite analizar en detalle la variabilidad de la fuente.

Durante la calibración de los flujos, debe considerarse la corrección que surge por la fracción de tiempo en que el instrumento no está capturando fotones, sino realizando la lectura del detector. A esta corrección se la llama corrección por tiempo muerto. También es importante verificar que la tasa de fotones no supere el máximo permitido por la telemetría del satélite, ni que el flujo de la fuente pueda producir un efecto de apilamiento de fotones (pile-up) considerable. Este efecto se produce cuando el detector recibe más de un fotón en el mismo píxel en una misma integración, lo que da lugar a una lectura errónea de la energía del fotón.

En caso de realizar una búsqueda de períodos, útil en el caso de los pulsares, se debe realizar la corrección por tiempo baricéntrico para el registro del arribo de los fotones. Además, es importante tener en cuenta el tiempo de lectura asociado al detector involucrado, que puede dar lugar a períodos espurios inherentes al instrumento y no a la fuente.

\section{Espectroscopía de rayos $\mathrm{X}$}

El ajuste de los espectros de rayos $\mathrm{X}$ a través de modelos expresados en función de la distribución de energía de los fotones originados en las fuentes estudiadas, constituye la técnica más utilizada para adquirir información de las propiedades físicas y químicas de las fuentes. Sin embargo, los parámetros espectrales que se derivan mediante el ajuste espectral dependen fuertemente de los modelos utilizados. La realidad física detrás de tales modelos es incierta, y en muchos casos los datos pueden ser descriptos satisfactoriamente por varios modelos físicos diferentes. En este sentido, es importante recalcar que, más allá de estas dificultades, los ajustes espectrales nos permiten obtener mayor conocimiento de la física de estos objetos, aunque todos los modelos sean en cierta medida simplificados.

Los modelos espectroscópicos en rayos X se construyen a partir de componentes individuales de dos tipos: aditivos (componentes de emisión, tales como un cuerpo negro o una línea espectral) o multiplicativos (debidos a agentes que modifican el espectro emitido, como una línea de absorción). En algunos casos los modelos pueden ser modificados de manera más compleja involucrando, por ejemplo, una convolución, que representa el suavizado producto del ensanchamiento de una línea de emisión por la velocidad del plasma asociado o una reflexión Compton. A lo largo de esta tesis utilizamos modelos como APEC (emisión de un plasma en equilibrio de ionización), PSHOCK (emisión de un plasma fuera del equilibrio de ionización), BBODYRAD (emisión de un cuerpo negro), POWERLAW (emisión no térmica) y PHABS (absorción interestelar).

Los ajustes espectrales se llevan a cabo usando el paquete XSPEC (Arnaud, 1996). En realidad, los detectores de rayos $\mathrm{X}$ no miden los espectros de las fuentes, sino que dan como resultado un conteo de fotones en los canales de energía disponibles, producto de la proyección del archivo de eventos en el espacio ortogonal a las coordenadas espaciales y temporales. Las cuentas observadas están relacionadas con el espectro original $f(E)$ (en unidades de fotones $\mathrm{cm}^{-2} \mathrm{~s}^{-1}$ ) por:

$$
C(I)=\int_{0}^{\infty} f(E) R(I, E) \mathrm{d} E
$$


donde $R(I, E)$ es la respuesta instrumental (que es una matriz que expresa la probabilidad de que un fotón con energía $E$ sea detectado por el canal $I$ ). En general no es posible obtener el espectro original a partir del observado, y lo que usualmente se hace es proponer un modelo basado en una serie de parámetros libres, y establecer la bondad del ajuste. Para ello XSPEC admite utilizar tanto estadísticas de $\chi^{2}$ (para estadísticas gaussianas, cuando hay suficientes fotones) como $C$ (para estadísticas poissonianas, para pocos fotones).

La manera de proceder es la siguiente: se cargan en XSPEC los espectros extraídos de la fuente y el fondo correspondiente. Luego se carga la respuesta del instrumento, que en XSPEC está formada por una matriz de respuesta $\left(\mathrm{RMF}^{26}\right)$ y un archivo auxiliar $\left(\mathrm{ARF}^{27}\right)$. Con esta información, XSPEC sustrae el fondo y normaliza el espectro teniendo en cuenta el tiempo de exposición y el área efectiva.

La expresión matemática del modelo utilizado se traduce en un vector de cuentas para los canales de energía involucrados que es comparado con el espectro observado, que es discreto. Para cada modelo $F(E)$ una tasa de cuentas es predicha a través de la Ecuación 1.65 que es comparada con la tasa observada usando un algoritmo de ajuste. La salida final es una serie de valores de los parámetros libres que mejor ajustan al espectro observado, así como sus rangos de confianza asociados, junto a un valor del test estadístico y los grados de libertad, que cuantifican la bondad del ajuste.

El espectro de la radiación emitida por una fuente es atenuado y modificado a medida que atraviesa su camino hasta el instrumento, debido a interacciones entre los fotones y la materia, ya sea del medio interestelar $\left(\mathrm{ISM}^{28}\right)$, intergaláctico o material intrínseco de la propia fuente. Para poder inferir el espectro original emitido por la fuente, el efecto causado por la absorción debe ser incorporado en el modelo espectral utilizado en el ajuste.

En el rango de 0.1-10 keV, el proceso que domina es la absorción fotoeléctrica, por la cual un átomo absorbe un fotón y libera un electrón con energía cinética igual a la diferencia entre la energía original del fotón y la energía de ligadura del electrón. A esta absorción contribuyen tanto el gas atómico y molecular como los granos de polvo, que dan lugar a una sección eficaz dependiente de la energía, $\sigma(E)$. Allí se pesan las contribuciones de los diferentes elementos químicos de acuerdo a sus abundancias relativas. La cantidad de material absorbente en la línea de la visual se expresa en proporción al número de átomos de $\mathrm{H}$, que es el elemento más abundante en el espacio, a través de la densidad columnar, $N_{\mathrm{H}}$, más allá de que justamente éstos prácticamente no intervengan en la absorción. Esta densidad columnar puede convertirse a los valores $A_{\mathrm{V}}=N_{\mathrm{H}} / 1.9 \times 10^{21} \mathrm{~cm}^{-2}$ usados en las bandas ópticas e infrarrojas. La intensidad observada, $I_{\mathrm{obs}}$, se relaciona con la intensidad de la fuente, $I_{\mathrm{em}}$, como:

$$
I_{\mathrm{obs}}(E)=e^{-\sigma(E) N_{\mathrm{H}}} I_{\mathrm{em}}(E)
$$

Si bien $\sigma(E)$ no es una función estrictamente monótona, ésta decrece con la energía, con un factor $\sim E^{-3}$, afectando principalmente las bandas por debajo de $\sim 2 \mathrm{keV}$. A energías mayores

\footnotetext{
${ }^{26}$ Del inglés, Response Matrix Function.

${ }^{27}$ Del inglés, Auxiliary Matrix Function.

${ }^{28}$ Del inglés, interstellar medium.
} 
a $10 \mathrm{keV}$, la dispersión debida a electrones libres y ligados puede volverse una nueva fuente de atenuación importante (Wilms et al., 2000).

\section{Colores en rayos $\mathrm{X}$}

Una técnica alternativa a la espectroscopía de rayos X es el análisis de los colores de la fuente, que equivale esencialmente al uso de filtros fotométricos en el óptico. Como los instrumentos de rayos X almacenan la energía de cada fotón detectado, la técnica de colores consiste en separar a los fotones en, por ejemplo, cuatro bandas anchas de energía, y calcular los cocientes entre ellas, que se denominan colores. Si bien no existe una forma estándar de definir esas bandas de energía, en la literatura se refiere a dichos colores como blandos o duros (soft o hard), según si corresponden a menores o mayores energías de los fotones, dentro del rango de detección del instrumento. Asimismo, al conteo de fotones en todo el rango de energía se lo denomina intensidad de rayos $\mathrm{X}$.

Los colores de las fuentes se grafican entonces en diagramas color-color o color-intensidad. De ellos surge que cada tipo de fuente realiza un recorrido particular en estos diagramas, hecho que se utiliza para poder clasificarlas. Si bien el análisis de la evolución de estos colores ofrece información mucho menos detallada que el análisis espectral, trae aparejada la ventaja de ser independiente del modelo utilizado para el ajuste espectral, siendo además preferible cuando las observaciones son realizadas con instrumentos de baja resolución, o cuando el número de fotones detectados es pequeño. Por la misma razón, el análisis de la evolución temporal de las fuentes en los diagramas color-color y color-intensidad, permite analizar su evolución espectral en una escala temporal más pequeña que los ajustes espectrales, ya que son necesarios menos fotones.

Desafortunadamente, los colores son específicos de cada instrumento, ya que para su cálculo no se considera su respuesta, que es única, lo que torna la comparación de los diagramas de distintos instrumentos muy compleja. Para mitigar este efecto, las cuentas suelen normalizarse en cada banda a través de la medición de fuentes estándares, tales como el Crab.

Cabe destacar que la combinación de las técnicas estándares presentadas aquí, da lugar a la posibilidad de desarrollar todo tipo de herramientas para extraer la mayor cantidad posible de información de las listas de eventos recopiladas por estos instrumentos. 
Parte II

Modelos teóricos de estrellas de neutrones 



\section{Breve descripción de la segunda parte}

En esta parte de la tesis presentamos el desarrollo de dos modelos teóricos basados en la evolución del campo magnético interno de NSs en dos escenarios extremos: el primero, referido a una NS que acreta materia proveniente del viento de su estrella compañera de gran masa en un sistema binario, y el segundo, que involucra a una NS altamente magnetizada.

En el Capítulo 2 introducimos los resultados obtenidos con un código numérico desarrollado para estudiar la evolución del campo magnético de NSs que experimentan un proceso de acreción en escalas de tiempo $\gtrsim 10^{7}$ años. Este trabajo tiene como objetivo intentar dar respuesta a la pregunta acerca de si NSs que acretan viento de una estrella compañera de gran masa en un sistema binario son capaces de formar jets relativistas, para lo cual, necesariamente, deberían poseer campos magnéticos relativamente débiles en su superficie. Parte de los resultados de este trabajo fueron publicados en un artículo de la revista Astronomy and Astrophysics (García et al., 2014).

Por otro lado, en el Capítulo 3, presentamos un modelo sencillo que podría explicar la disminución repentina de la velocidad de rotación observada en el magnetar AXP 1E 2259+586, fenómeno conocido como anti-glitch. En el escenario propuesto, este cambio se explica naturalmente a partir de un re-acomodamiento de la estructura de la estrella hacia un estado de equilibrio, en completo acuerdo con la energética asociada al evento. Los resultados de este trabajo fueron publicados en una Letter de la revista Monthly Notices of the Royal Astronomical Society (García \& Ranea-Sandoval, 2015). 


\section{Capítulo 2}

\section{Evolución del campo magnético en estrellas de neutrones acretantes}

\subsection{Resumen}

En el mecanismo magneto-centrífugo para la formación de jets, se supone que NSs acretantes son capaces de producir jets relativistas únicamente si el campo magnético en su superficie es suficientemente débil $\left(B \sim 10^{8} \mathrm{G}\right)$. Sin embargo, sabemos que la manifestación más común de estas estrellas es en forma de pulsares, con campos magnéticos típicos del orden de $B \sim 10^{12} \mathrm{G}$. Por lo tanto, si el campo magnético de estas estrellas tiene una intensidad de ese orden al nacer, debe decaer considerablemente antes de que pueda volverse una fuente capaz de emitir jets en un sistema binario. En este capítulo estudiamos la evolución del campo magnético de una NS que acreta materia del viento de una estrella compañera de gran masa, con el objetivo de poder poner cotas a la tasa de acreción, y conocer las condiciones de la microfísica de la corteza, que resulten necesarias para hacer posible la formación de jets. Para ello, resolvemos la ecuación de inducción para la difusión y advección de un campo magnético confinado a la corteza de una NS, bajo las hipótesis de acreción esférica que habilita un tratamiento unidimensional, a partir del desarrollo de un código numérico propio que incorpora el estado del arte en la microfísica, incluyendo perfiles de evolución térmica consistentes con una serie de escenarios diferentes basados en las condiciones de superfluidez del núcleo de la estrella. Bajo estas hipótesis, mostramos que en este escenario, el decaimiento del campo magnético en escalas de tiempo largas esta gobernado principalmente por la tasa de acreción, mientras que el contenido de impurezas y la evolución térmica de la estrella juegan un papel secundario. En general, para tasas de acreción $\dot{M}>10^{-10} \mathrm{M}_{\odot}$ año ${ }^{-1}$, el campo magnético puede decaer hasta cuatro ordenes de magnitud en $t \sim 10^{7}$ años, que es la escala de tiempo impuesta por la edad de su compañera de gran masa. Finalmente, a modo de corolario, discutimos la posibilidad de que en sistemas binarios compuestos por NSs que acretan material del viento de una estrella compañera de gran masa, como es el caso de los SFXTs, puedan formar jets, convirtiéndose en una hipotética fuente de rayos $\gamma$ en nuestra galaxia. 


\subsection{Introducción}

Una nueva clase de fulguraciones transitorias en rayos $\mathrm{X}$ fue descubierta a través de observaciones realizadas con el satélite INTEGRAL a lo largo del plano Galáctico. Estas fuentes, denominadas SFXTs, se piensa son originadas en sistemas binarios compuestos por estrellas supergigantes $\mathrm{O}$ o $\mathrm{B}$ y un objeto compacto (una NS o un agujero negro). Estas binarias presentan fulguraciones que, en general, duran desde horas hasta días, alcanzando luminosidades $L_{\mathrm{X}} \sim 10^{36}-10^{37} \mathrm{erg} \mathrm{s}^{-1}$ para luego decaer hasta niveles de quietud de $L_{\mathrm{X}} \sim 10^{32} \mathrm{erg} \mathrm{s}^{-1}$ (Negueruela et al., 2006; Pellizza et al., 2006; Sguera et al., 2005). Producto de acreción residual sobre el objeto compacto, suelen permanecer la mayor parte del tiempo en un estado intermedio con luminosidades $L_{\mathrm{X}} \sim 10^{33}-10^{34} \mathrm{erg} \mathrm{s}^{-1}$ (Sidoli et al., 2008).

Actualmente, la clase de los SFXTs posee una decena de miembros, identificados a partir de la asociación de la fuente transitoria en los rayos X con sus contrapartidas ópticas, cuyos espectros corresponden a estrellas supergigantes azules. Además, hay una serie de candidatas a miembros de la clase, que permanecen sin ser confirmadas, ya que sus contrapartidas en el óptico o infrarrojo no han sido detectadas (ver Sidoli, 2011). En al menos cuatro de estos SFXTs, el descubrimiento de pulsaciones periódicas en los rayos X, que cubren desde los 4.7 hasta los 228 s, confirma la presencia de una NS. Asimismo, también han sido determinados algunos períodos orbitales, que abarcan desde los 3.3 hasta los 165 días. Tres modelos fueron propuestos para explicar el mecanismo de fulguraciones rápidas (ver Sidoli, 2009): (i) vientos inhomogéneos esféricamente simétricos, donde las fulguraciones de rayos $\mathrm{X}$ son producidas cuando un grumo denso es acretado por el objeto compacto (Ducci et al., 2009; in't Zand, 2005; Negueruela et al., 2008; Walter \& Zurita Heras, 2007); (ii) vientos anisotrópicos, donde las fulguraciones ocurren cuando el objeto compacto cruza una componente ecuatorial densa y lenta, aumentando la tasa de acreción (Sidoli et al., 2007); (iii) mecanismos de compuerta, donde la acreción es inhibida por una barrera centrífuga o magnética (Bozzo et al., 2008; Grebenev \& Sunyaev, 2007), lo que requiere una NS con un campo magnético muy intenso $\left(B \sim 10^{14}-10^{15} \mathrm{G}\right)$ y períodos de rotación muy largos $\left(P_{\mathrm{s}} \sim 10^{3} \mathrm{~s}\right)$. En estos SFXTs aún no ha sido detectada actividad relacionada con la presencia de un magnetar. A partir de la detección de una línea ciclotrón en el espectro de SFXT IGR J18483-0311, suponiendo un origen electrónico, pudo estimarse un campo magnético relativamente bajo $\left(B \sim 10^{11} \mathrm{G}\right)$, aunque la presencia de un magnetar no puede descartarse si la línea es causada por protones, para los cuales $B \sim 5 \times 10^{14} \mathrm{G}$.

Cuatro fuentes de radiación $\gamma$ transitorias no identificadas, AGL J2022+3622, 3EG J18370423, 3EG J1122-5946, y AGL J1734-3310, coinciden espacialmente con tres de estos SFXTs (o candidatos): IGR J20188+3647, AX J1841.0-0536, IGR J11215-5952 (Sguera, 2009), y un SFXT intermedio: IGR J17354-3255 (Sguera et al., 2011). Esta relación aumenta las chances de que estas fuentes sean capaces de producir radiación por encima de los $100 \mathrm{MeV}$. En un trabajo reciente, Sguera et al. (2009) desarrollaron un modelo para la producción de radiación $\gamma$ de SFXT AX J1841.0-0536 basados en la hipótesis de que la radiación de altas energías se origina en el enfriamiento de partículas relativistas aceleradas en un flujo colimado eyectado por la NS. A pesar de que la emisión de jets por NS ha sido confirmada en algunos LMXBs, este tipo de eyecciones 
no ha podido ser confirmada aún en HMXBs que albergan NSs, a pesar que esta posibilidad fue discutida para el caso de la emisión $\gamma$ observada en HMXB LS I +61 303 (Romero et al., 2007).

El mecanismo magneto centrífugo para la formación de jets en NSs acretantes requiere que el material acretado arrastre consigo las líneas de campo magnético cerca de la superficie del objeto compacto. La presión del fluido en esta región debe ser mayor que la presión ejercida por el campo magnético de la NS. Para ello, el campo magnético no debe superar $B \sim 10^{8} \mathrm{G}$ (Massi \& Kaufman Bernadó, 2008). En el caso de los LMXB con NSs viejas ( $\gtrsim 10^{9}$ años), el campo magnético ha tenido tiempo suficiente para decaer varios órdenes de magnitud desde valores del orden de $\sim 10^{12} \mathrm{G}$ para el campo magnético inicial. Por el contrario, en HMXBs, las escalas de tiempo asociadas a la evolución de la estrella de gran masa son bastante menores ( $10^{7}$ años). Sin embargo, este tipo de estrellas presentan vientos intensos fuertemente inhomogéneas (Negueruela, 2010; Owocki \& Cohen, 2006; Runacres \& Owocki, 2005) que acrecientan el proceso de acreción sobre la NS pudiendo disparar un decaimiento acelerado del campo magnético en la superficie de la NS gracias a la advección de las corrientes que originan su campo magnético hacia el interior de la estrella en un proceso de enterramiento (ver el trabajo pionero de Bisnovatyi-Kogan \& Komberg, 1974). Además, el incremento de las impurezas de la corteza debido al proceso de acreción podrían incrementar su resistividad, aumentando el decaimiento difusivo del campo.

En este trabajo nos proponemos explorar cuáles son las condiciones necesarias que deben ser satisfechas por una NS inmersa en un HMXB para poder desarrollar un decaimiento de su campo magnético superficial desde un valor inicial de $B \sim 10^{12} \mathrm{G}$ hasta valores finales del orden de $B \lesssim 10^{8} \mathrm{G}$, que podrían permitir la formación y eyección de jets en estos sistemas. Para ello, utilizamos un modelo unidimensional para una NS que acreta con simetría esférica, incorporando el estado del arte en la microfísica asociada a la conductividad eléctrica y a las curvas de enfriamiento. Consideramos, además, escenarios cualitativamente diferentes en términos de la tasa de acreción, superfluidez en el núcleo, y contenido de impurezas en la corteza, para lo cual resolvemos la ecuación de inducción para la evolución del campo magnético con condiciones iniciales y de borde apropiadas, a lo largo de una escala de tiempo que cubre los $10^{7}$ años. Con esta aproximación al problema, pretendemos establecer cotas que sirvan a modelos más realistas de la evolución magneto-térmica de estas estrellas (ver Viganò et al., 2012).

\subsection{Modelo de estrella de neutrones}

\subsubsection{Corteza acretada}

Para poder estudiar la evolución del campo magnético, comenzamos por construir un modelo de NS usando una EoS que representa tanto la corteza como su interior, basada en la interacción nuclear efectiva SLY (Douchin \& Haensel, 2001), y considerando una composición de la corteza modificada por el material acretado (Haensel \& Zdunik, 2008). A bajas densidades en la corteza exterior $\left(\rho \sim 10^{10} \mathrm{gr} \mathrm{cm}^{-3}\right)$ la composición alterada de la corteza presenta núcleos con números atómicos bajos $(Z<20)$, mientras que para materia catalizada al equilibrio, los números atómicos suelen estar en el rango de 40 a 50. A densidades intermedias, la red pasa a estar compuesta por 
Tabla 2.1: Densidad central $\rho_{\mathrm{c}}$, masa $M$, radio $R_{\mathrm{NS}}$ y espesor de la corteza $\Delta R$ para los modelos de baja masa (LM) y alta masa (HM) utilizados.

\begin{tabular}{ccccc}
\hline \hline $\begin{array}{c}\text { Estrella de neutrones } \\
\text { Modelo }\end{array}$ & $\begin{array}{c}\rho_{\mathrm{c}} \\
{\left[\mathrm{g} \mathrm{cm}^{-3}\right]}\end{array}$ & $\begin{array}{c}M \\
{\left[M_{\odot}\right]}\end{array}$ & $\begin{array}{c}R_{\mathrm{NS}} \\
{[\mathrm{km}]}\end{array}$ & $\begin{array}{c}\Delta R \\
{[\mathrm{~km}]}\end{array}$ \\
\hline Baja masa (LM) & $9.9 \times 10^{14}$ & 1.4 & 11.72 & 0.93 \\
Alta masa (HM) & $1.4 \times 10^{15}$ & 1.8 & 11.34 & 0.59 \\
\hline
\end{tabular}

núcleos con números másicos $A \lesssim 100$ bastante menos que los valores típicos correspondientes a NS aisladas $(A \sim 300)$. A densidades altas en la corteza interna $\left(\rho>10^{13} \mathrm{gr} \mathrm{cm}^{-3}\right)$, la composición del material acretado es similar al caso no-acretado, ya que el gas de neutrones interactúa con la red iónica, volviendo al efecto causado por la acreción mucho menos significativo que en las regiones exteriores.

Para investigar las diferencias que podrían deberse a las discrepancias en la estructura interna de las NSs, estudiamos dos configuraciones extremas: una NS típica de $1.4 \mathrm{M}_{\odot}$, que llamamos modelo (LM), y otra de $1.8 \mathrm{M}_{\odot}$, que denominamos (HM). En la Tabla 2.1 presentamos las características más importantes de cada una de las configuraciones.

Para la EoS elegida, la interfase entre la corteza y el núcleo se encuentra a $\rho_{\mathrm{CC}}=0.46 \rho_{0}$, donde $\rho_{0}=2.8 \times 10^{14} \mathrm{~g} \mathrm{~cm}^{-3}$ es la densidad de saturación nuclear. El espesor de la corteza, definido como la distancia desde dicha interfase hasta la superficie de la NS, mide alrededor de $\sim 1 \mathrm{~km}$ en el modelo LM, siendo $\sim 60 \%$ más delgado en el modelo HM. Este espesor representa la escala característica de confinamiento del campo magnético, ya que éste es originado en las corrientes que circulan en la corteza.

\subsubsection{Curvas de enfriamiento}

Suponemos una corteza espacialmente isotérmica caracterizada por una temperatura que evoluciona en el tiempo siguiendo las curvas de enfriamiento presentadas en la Figura 2.1, que fueron calculadas para dos modelos de NS consistentes (Aguilera et al., 2008).

Para poder analizar cualitativamente los efectos introducidos por la evolución térmica en la evolución del campo magnético, incorporamos dos casos extremos para la superfluidez de los neutrones (SF) caracterizados por la onda- $p$ (en el estado ${ }^{3} P_{2}$ ) en el núcleo de la NS: superfluidez fuerte (modelo strong $S F$ ), donde consideramos temperaturas críticas muy altas, $T_{c} \approx 6 \times 10^{9} \mathrm{~K}, \mathrm{y}$ en el otro extremo, ausencia de superfluidez para este nucleón (modelo no $S F$ ). En ambos modelos suponemos superfluidez en la onda- $s$ correspondiente a los neutrones de la corteza y los protones del núcleo (con temperaturas críticas $T_{c} \approx 8 \times 10^{9} \mathrm{~K}, \approx 6 \times 10^{9} \mathrm{~K}$, respectivamente). Es importante notar que estas correlaciones dejan una impronta mucho más leve sobre las curvas de enfriamiento que la debida a los neutrones en el núcleo.

Para $t \lesssim 10^{5}$ años, el apareamiento de neutrones en la onda- $p$ produce un enfriamiento fuertemente desacelerado respecto del caso no superfluido. En esta primera fase, la estrella más masiva sufre un enfriamiento mucho más veloz debido a la emisión eficiente de neutrinos producto del 


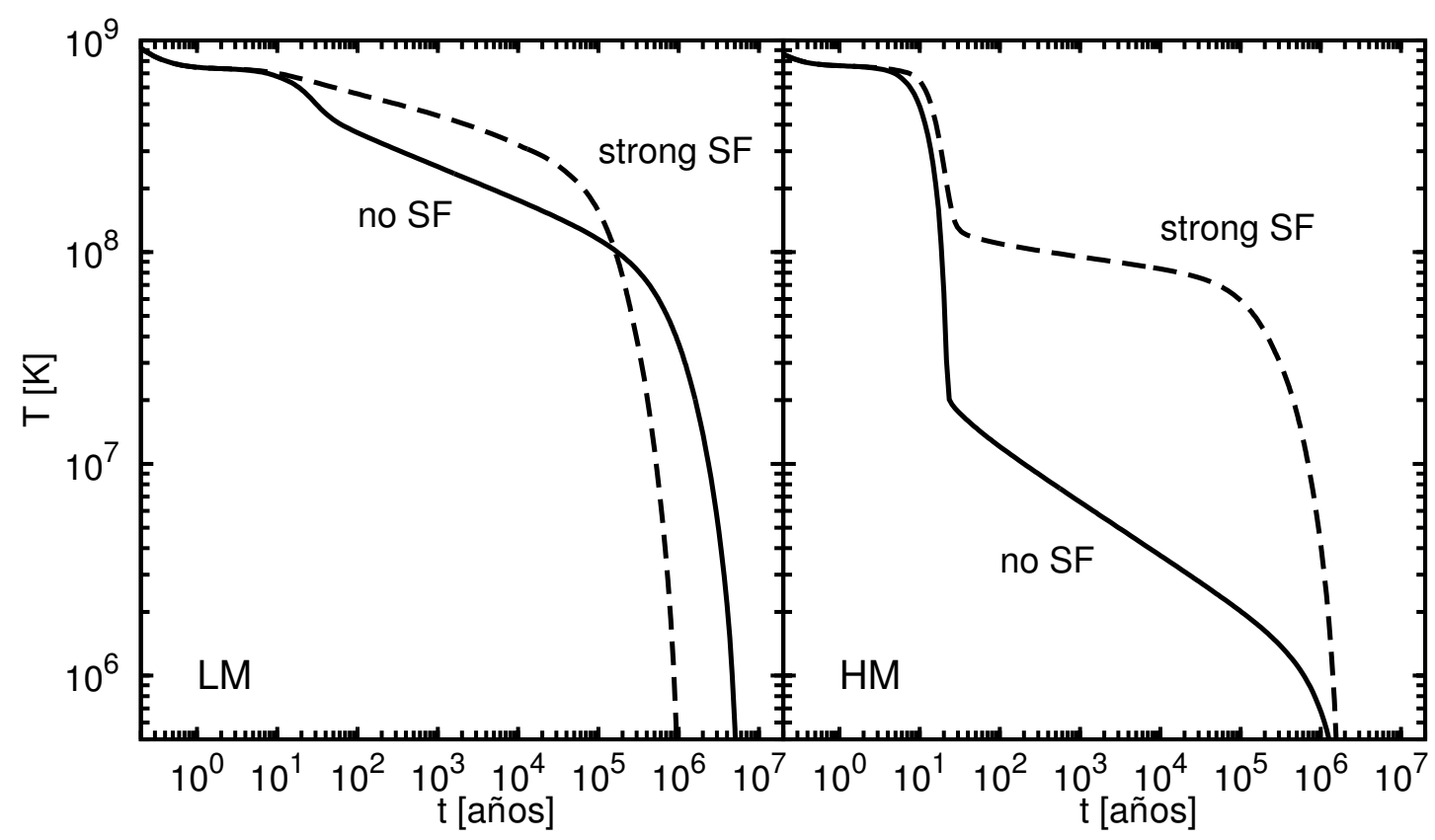

Figura 2.1: Curvas de enfriamiento adoptadas (Aguilera et al., 2008). El panel izquierdo (derecho) muestra la evolución térmica para dos casos casos extremos en la superfluidez del núcleo del modelo LM (HM).

proceso Urca directo. Cuando la temperatura es suficientemente baja (a $t>10^{5}$ años), la emisión de fotones domina el enfriamiento y la supresión del calor específico debido a la superfluidez da como resultado un enfriamiento acelerado, con temperaturas finales $T<10^{5} \mathrm{~K}$ en escalas de tiempo $t<10^{7}$ años.

\subsubsection{Evolución del campo magnético}

Bajo las hipótesis de alta conductividad eléctrica y velocidades de fluido no relativistas, la evolución del campo magnético en la corteza de la NS puede describirse por la ecuación de inducción de la magnetohidrodinámica:

$$
\frac{\partial \mathbf{B}}{\partial t}=-\frac{c^{2}}{4 \pi} \nabla \times\left(\frac{1}{\sigma} \nabla \times \mathbf{B}\right)+\nabla \times(\mathbf{v} \times \mathbf{B}),
$$

donde $c$ es la velocidad de la luz, $\mathbf{v}$ es la velocidad del fluido, y $\sigma$ es la conductividad eléctrica.

La ecuación diferencial en derivadas parciales (2.1) para la evolución del campo magnético B involucra dos térmicos. El primero es el término difusivo, que depende esencialmente de la conductividad eléctrica. El segundo es el término advectivo o convectivo, que se origina en el arrastre de las líneas de campo magnético causado por el movimiento del fluido que alberga sus fuentes.

Como fuera demostrado en el Capítulo 1, suponiendo una configuración dipolar para el campo magnético, el potencial vector puede escribirse $\mathbf{A}=\left(0,0, A_{\phi}\right)$, donde $A_{\phi}=s(r, t) \sin \theta / r$. Aquí $r$ es la 
coordenada radial y $\theta$ y $\phi$ son los ángulos polar y acimutal, respectivamente. Luego, suponiendo un flujo de materia acretada esféricamente simétrico, la ecuación de inducción puede reducirse a una ecuación unidimensional en derivadas parciales para la función de Stokes, $s(r, t)$ :

$$
\frac{\partial s}{\partial t}=\frac{c^{2}}{4 \pi \sigma}\left(\frac{\partial^{2} s}{\partial r^{2}}-\frac{2 s}{r^{2}}\right)-v_{r} \frac{\partial s}{\partial r}
$$

A partir de la ecuación de continuidad, la velocidad del fluido, $v_{r}(r)$, puede escribirse en función de la tasa de acreción, $\dot{M}$, que es un parámetro libre en nuestro modelo:

$$
v_{r}(r)=-\frac{\dot{M}}{4 \pi r^{2} \rho(r)},
$$

donde $\rho(r)$ es el perfil de densidad de la corteza de la NS. Es importante destacar que para las tasas de acreción e intensidades de campo magnético consideradas aquí, sería esperable que la acreción se desarrollara en forma de columnas entorno a los polos magnéticos, involucrando sólo una fracción de la superficie de la NS. Este hecho motivaría un tratamiento bidimensional para el flujo de materia y su efecto sobre el área acretada, que se encuentra fuera de nuestro alcance. Por el contrario, preferimos enfocarnos en los aspectos cualitativos del decaimiento del campo magnético que son esperables también en un modelo de acreción columnar.

Para resolver la ecuación diferencial (2.2), es necesario imponer condiciones de contorno e iniciales apropiadas para el problema. En la superficie, la condición de contorno proviene de pegar la geometría del campo magnético interior con la solución de vacío dipolar para el campo magnético exterior. Siguiendo el trabajo de Konar \& Bhattacharya (1997), la condición de contorno interior es obtenida suponiendo que la conductividad eléctrica en el núcleo de la NS es varios órdenes de magnitud superior a la de la corteza. Para la condición inicial, suponemos que las corrientes fuente del campo magnético son expulsadas del núcleo de la estrella al formarse la NS por los protones superconductores, y por lo tanto el campo magnético queda confinado a la corteza (Geppert \& Urpin, 1994):

$$
s(r, t=0)=\left\{\begin{array}{cll}
0 & \text { if } & r<r_{\mathrm{i}} \\
\left(\frac{r-r_{\mathrm{i}}}{R_{\mathrm{NS}}-r_{\mathrm{i}}}\right)^{2} & \text { if } & r_{\mathrm{i}}<r \leq R_{\mathrm{NS}}
\end{array},\right.
$$

donde $r_{\mathrm{i}}$ es la distancia radial para la expulsión inicial del campo magnético, y donde hemos normalizado la función de Stokes en la superficie, $s\left(R_{\mathrm{NS}}, t=0\right)=1$, por simplicidad. Bajo estas hipótesis, el campo magnético en la superficie de la NS a un tiempo arbitrario, $t$, puede obtenerse a partir del valor de la función de Stokes, $B\left(R_{\mathrm{NS}}, t\right)=s\left(R_{\mathrm{NS}}, t\right) \cdot B\left(R_{\mathrm{NS}}, t=0\right)$, donde $B\left(R_{\mathrm{NS}}, t=0\right)$ es la intensidad del campo magnético superficial que fijamos al valor canónico de $10^{12} \mathrm{G}$.

Las conductividades eléctricas y térmicas en la corteza de la NS juegan un rol fundamental tanto en la evolución magnética como en la térmica. En particular, la conductividad eléctrica gobierna la evolución del campo magnético a través del término difusivo de la Ecuación 2.1 de inducción. En la corteza de la NS los principales portadores de carga son los electrones, por lo que el coeficiente de transporte, $\sigma$, es determinado teniendo en cuenta todos los procesos de dispersión 
con ellos: iones (p), fonones (ph), e impurezas (Q). A partir del código de dominio público ${ }^{1}$ basado en Potekhin (1999), tomamos las conductividades eléctricas no cuantizadas incorporando las modificaciones introducidas por Chugunov (2012). Estas rutinas dan $\sigma$ como función de la temperatura, $T$, densidad, $\rho$, intensidad de campo magnético, $B$, y la composición de la corteza, caracterizada por los números atómicos y másicos, $(Z, A)$, así como el número másico auxiliar, que incorpora el gas de neutrones libres, $A^{*}$, y el parámetro que caracteriza el contenido de impurezas, $Q=Z_{\text {imp }}^{2}$.

El contenido de impurezas está definido como la medida de la dispersión de carga en la red iónica, $Q=\frac{1}{n_{i}} \sum_{n^{\prime}} n^{\prime}\left(Z-Z^{\prime}\right)^{2}$, donde $n_{i}$ es la densidad numérica del ión dominante de carga $Z$ y $n^{\prime}$ son las densidades numéricas de las especies intercaladas de cargas $Z^{\prime}$. La conductividad asociada con $Q$ domina a temperaturas bajas, cuando la conductividad debida a la dispersión de fonones se vuelve despreciable. En nuestro trabajo, suponemos dos valores extremos para el parámetro de impurezas en la corteza exterior: $Q=0.1$, que representa un cristal prácticamente ordenado, y $Q=5$, para un alto grado de impurezas. En la corteza interna, más allá de la densidad de deriva nuclear (ND) $\left(\rho>\rho_{\mathrm{ND}}=4.2 \times 10^{11} \mathrm{~g} \mathrm{~cm}^{-3}\right)$, la presencia de un gas de neutrones libres que interaccionan con la red iónica impidiendo la formación de impurezas, por lo que fijamos las impurezas a $Q=0$.

En la Figura 2.2 mostramos la conductividad eléctrica como función de la densidad en el interior de la NS considerando dos temperaturas: $T=10^{6}-10^{8} \mathrm{~K}$ y dos valores del parámetro de impurezas en la corteza externa: $Q=0.1-5.0$. El recuadro en la esquina superior derecha de la figura muestra en detalle el salto en la conductividad eléctrica próximo a la densidad de deriva neutrónica (ND). Más allá de la interfase entre la corteza y núcleo, la conductividad eléctrica es muy alta, varios órdenes de magnitud por sobre los correspondientes a la corteza externa.

En la corteza interna, donde hemos fijado $Q=0$, la conductividad depende fuertemente de la temperatura. Para una temperatura de $T=10^{6} \mathrm{~K}$, la conductividad presenta un gradiente pronunciado, variando desde $\sigma \gg 10^{35} \mathrm{~s}^{-1}$ hasta $10^{33} \mathrm{~s}^{-1}$ a la densidad ND. Sin embargo, para una temperatura mayor, de $10^{8} \mathrm{~K}$, el gradiente se hace más leve, pasando tan solo de $10^{27}$ a $10^{24} \mathrm{~s}^{-1}$. El salto en la interfase entre la corteza y el núcleo disminuye a medida que la temperatura es más baja.

A pesar que en la corteza externa las diferencias son menos pronunciadas que en la corteza interna, la conductividad no sólo presenta un gradiente importante, sino también una fuerte dependencia con la temperatura y el parámetro de impurezas. Para una temperatura de $10^{8} \mathrm{~K}$, la conductividad eléctrica varía desde $\sim 10^{24} \mathrm{~s}^{-1}$ a la densidad ND hasta $10^{20} \mathrm{~s}^{-1}$ en la superficie de la NS. En este caso, considerando un alto contenido de impurezas, dado por $Q=5.0$, se observa un pequeño salto en la conductividad a la densidad ND y, para densidades $\rho<10^{10} \mathrm{~g} \mathrm{~cm}^{-3}$, la conductividad resulta independiente del parámetro $Q$. Para una temperatura menor, $T=10^{6} \mathrm{~K}$, la conductividad en la corteza externa se comporta de una manera muy diferente. Cuando el contenido de impurezas es bajo $(Q=0.1), \sigma$ varía desde $3 \times 10^{25} \mathrm{~s}^{-1}$ a la densidad ND hasta $\sim 10^{21} \mathrm{~s}^{-1}$ en la superficie, permaneciendo 1-3 órdenes de magnitud respecto a los valores correspondientes

\footnotetext{
${ }^{1}$ http://www . ioffe.ru/astro/conduct/index.html
} 


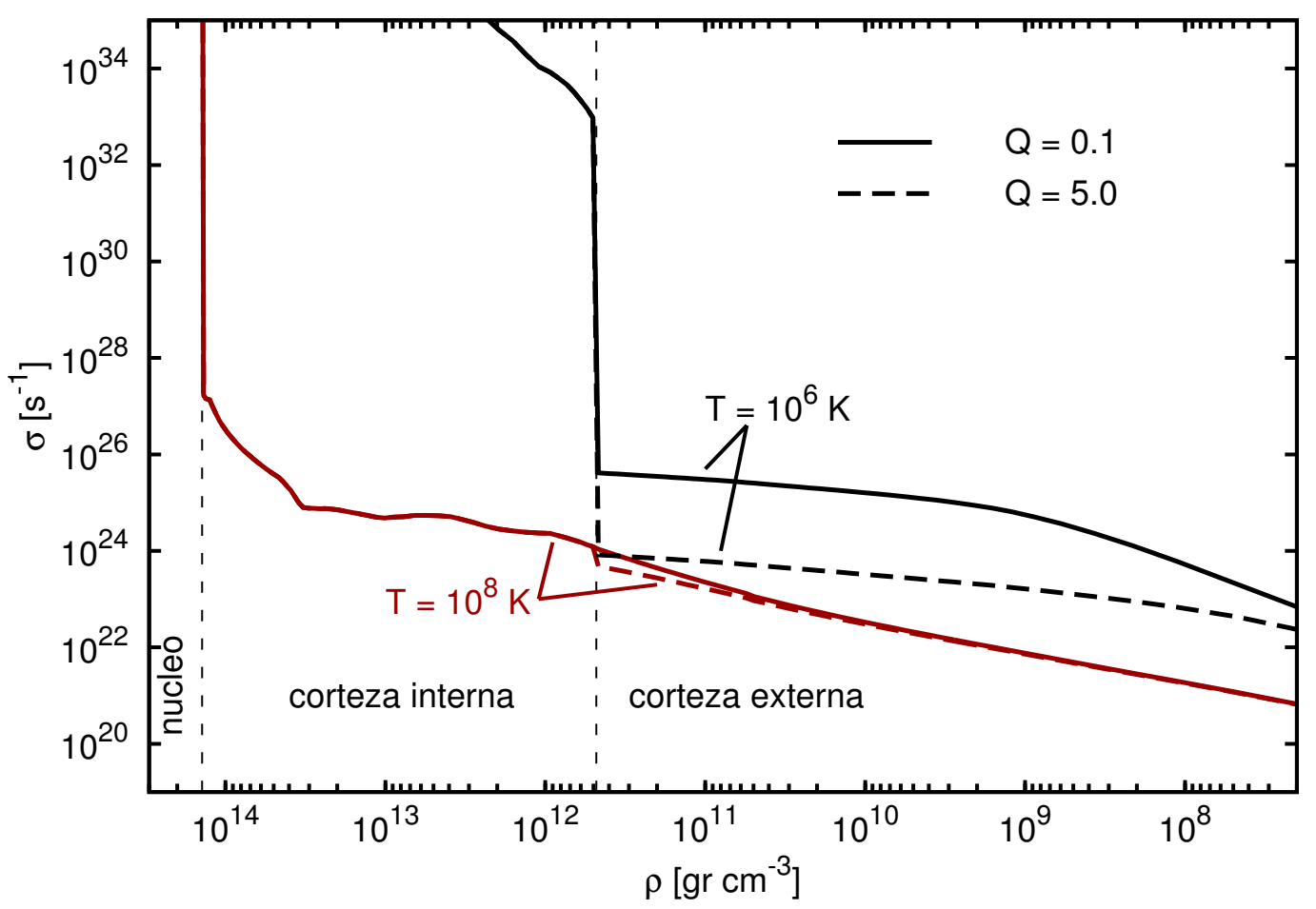

Figura 2.2: Conductividad eléctrica, $\sigma$, en la corteza de la NS para dos temperaturas $\left(10^{6}\right.$ y $\left.10^{8} \mathrm{~K}\right)$ y los dos valores del parámetro de impurezas, $Q=0.1$ y 5.0$)$.

a $T=10^{8} \mathrm{~K}$ en toda la corteza externa. Para un contenido de impurezas alto, dado por $Q=5.0$, la conductividad resulta al menos un orden de magnitud más baja para $\rho \gtrsim 10^{8} \mathrm{gr} \mathrm{cm}^{-3}$, volviéndose comparable al caso de bajas impurezas cerca de la superficie de la estrella. A bajas temperaturas, las impurezas se tornan prácticamente el único blanco efectivo para los procesos de dispersión de electrones, por lo que el salto a la densidad ND, crece fuertemente a medida que la estrella se enfría.

\subsection{Resultados numéricos}

Para resolver la ecuación de inducción de la función de Stokes (Ecuación 2.2), utilizamos un método de división de operadores que combina un esquema implícito de Crank-Nicolson para el término difusivo con un mecanismo explícito para el término convectivo o advectivo. Luego de sucesivas pruebas en el paso espacial, fijamos una grilla de 200 puntos entre la superficie de la NS, $r=R_{\mathrm{NS}}$, y el límite interior, a $r=10 \mathrm{~km}$, fijo a unos $\sim 0.75 \mathrm{~km}$ dentro del núcleo de la estrella, para satisfacer la condición de contorno interior. Para el término difusivo adoptamos un paso temporal que se incrementa como $\Delta t=t / 200$. En cada paso difusivo, resolvemos el término convectivo dividiendo el intervalo de tiempo en pasos más cortos, de manera de satisfacer la condición CFL 
(Courant et al., 1928). Para calcular el término difusivo, nuestro código numérico invoca las rutinas de dominio público dadas por Potekhin (1999), para el cálculo de la conductividad eléctrica, $\sigma$, en cada punto de la grilla espacial. La densidad, $\rho$, y la composición de la corteza, $(A, Z)$, son tomadas de la EoS y la temperatura, $T$, de los perfiles de evolución térmica (ver Subsección 2.3.2). El contenido de impurezas, $Q=Z_{\text {imp }}^{2}$, y la tasa de acreción, $\dot{M}$, son parámetros fijos en cada una de nuestras simulaciones.

Presentamos primero la evolución del perfil de la función de Stokes en la corteza de la NS tanto para el modelo LM (Figura 2.3) como para el modelo HM (Figura 2.4), considerando cuatro valores diferentes de la tasa de acreción, $\dot{M}_{-10}$, escrita en unidades de $10^{-10} \mathrm{M}_{\odot} \mathrm{yr}^{-1}$. Al primer $t=1$ año, el perfil de la función de Stokes resulta prácticamente idéntico a la distribución inicial dada por $s(r, t=0)$ (ver Ecuación 2.4). En la primera etapa $\left(t<10^{5}\right.$ años), el campo magnético es re-ordenado hacia la corteza interna, ya que la conductividad es mayor que en la corteza externa, reduciéndose en un factor $\sim 10$ en la superficie, $R_{\mathrm{NS}}$. Durante toda esta primera fase, los perfiles de la función de Stokes permanecen en todos los casos como funciones monótonamente crecientes con el radio, como la distribución inicial. Para tasas de acreción $\dot{M}<10^{-10} \mathrm{M}_{\odot}$ año ${ }^{-1}$, el decaimiento permanece siendo uniforme en toda la corteza, y la función de Stokes alcanza valores próximos a $\sim 10^{-2}$ en la superficie de la estrella en escalas de tiempo de $\sim 10^{7}$ años. Cuando la contribución del término convectivo es comparable a la de la componente difusiva, el arrastre del campo magnético hacia el interior de la NS para tasas de acreción $\dot{M} \gtrsim 10^{-10} \mathrm{M}_{\odot}$ año ${ }^{-1}$ se torna suficientemente fuerte para producir un proceso de enterramiento, dando lugar a que la intensidad del campo magnético en la superficie sea menor que en la corteza interna, donde la conductividad es varios órdenes de magnitud mayor.

En las figuras 2.5 y 2.6 presentamos la evolución del campo magnético en la superficie de las NSs correspondientes a los modelos LM y HM como función de la tasa de acreción, $\dot{M}$, considerando los dos escenarios propuestos para la superfluidez (no $S F$ y strong $S F$ ) y los dos contenidos de impurezas $(Q=0.1$ y $Q=5.0)$.

Para el modelo LM (Figura 2.5), durante la primera etapa $\left(t<10^{5}\right.$ años), mientras que la estrella permanece caliente $\left(T \gtrsim 10^{8} \mathrm{~K}\right)$, el decaimiento del campo magnético resulta similar en todos los casos, ya que su evolución resulta dominada por el proceso difusivo. Una vez que la estrella se enfría, el proceso de acreción comienza a gobernar el decaimiento del campo en la superficie producto del arrastre del campo hacia las capas interiores. Luego, las curvas de decaimiento se separan entre ellas en función del valor de $\dot{M}$. Además, pasados $t \sim 10^{6}$ años, también comienza a jugar un papel importante el contenido de impurezas. Para un bajo grado de impurezas, dado por $Q=0.1$, cuando la temperatura es suficientemente baja $\left(T \lesssim 10^{6} \mathrm{~K}\right)$, la conductividad en la corteza externa se torna $\sim 2$ órdenes de magnitud mayor que en el caso de alto grado de impurezas, $Q=5.0$. Por lo tanto, el campo magnético se reduce más rápidamente para $Q=0.1$, ya que el proceso de advección se vuelve más significativo cuando la difusión disminuye. Para $Q=5.0$, el campo magnético es difundido suavemente en la corteza externa, evitando la aparición de gradientes que permitan acelerar su enterramiento hacia el interior. Sin embargo, para tasas de acreción suficientemente elevadas, $\dot{M} \sim 5 \times 10^{-10} \mathrm{M}_{\odot}$ año ${ }^{-1}$, el campo magnético en la superficie decae indefectiblemente por debajo de $10^{8} \mathrm{G}$ en menos de $2 \times 10^{7}$ años. Lo contrario 


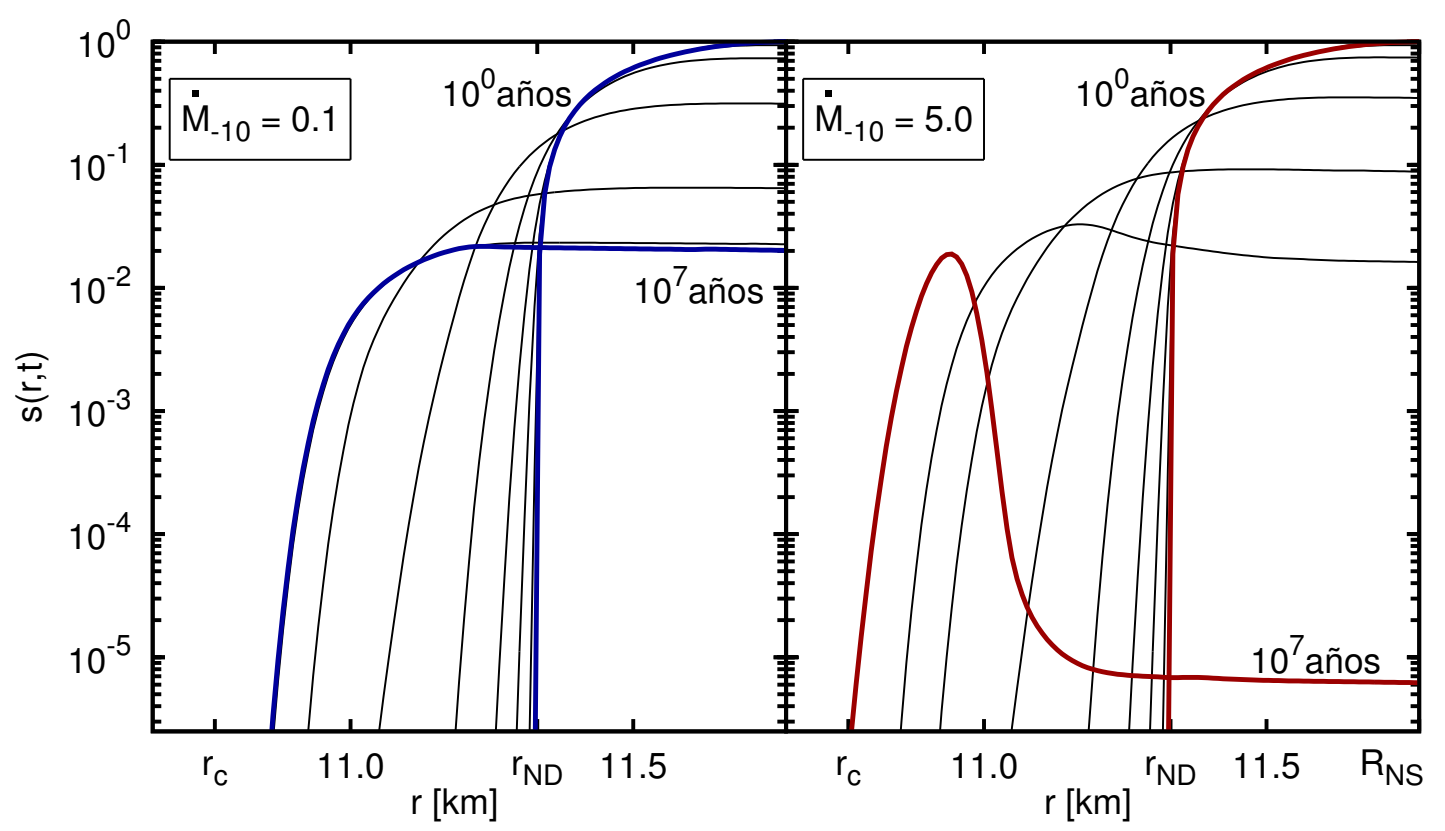

Figura 2.3: Evolución de la función de Stokes en la corteza del modelo LM. Cada panel corresponde a una tasa de acreción diferente. En ambos paneles, cada curva (de arriba abajo en la superficie de la NS, $\left.R_{\mathrm{NS}}\right)$ corresponde a $t=1,10^{1}, 10^{2}, 10^{3}, 10^{4}, 10^{5}, 10^{6}$, y $10^{7}$ años. En todos los casos, fijamos $Q=5$ y el modelo no $S F$.

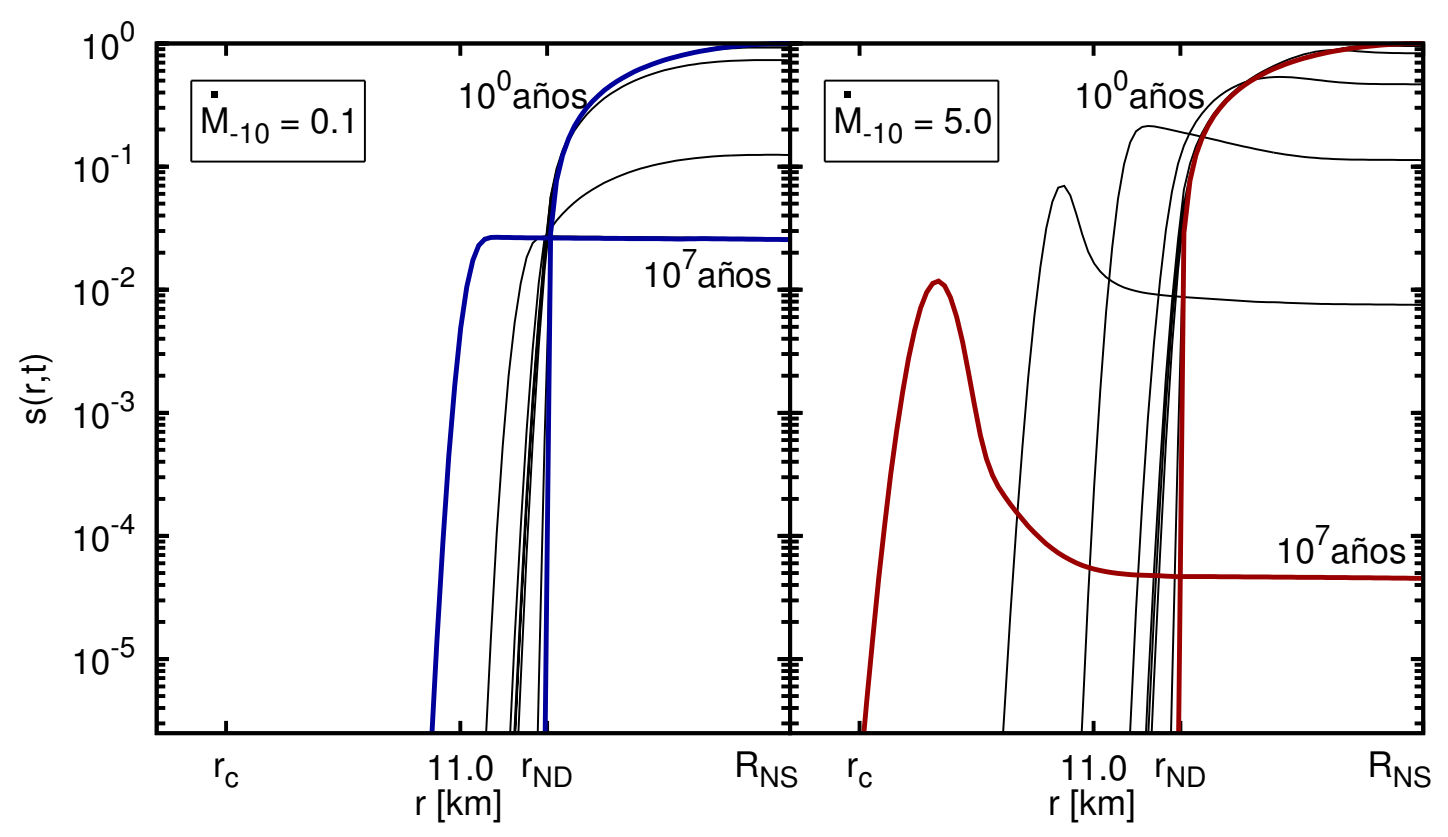

Figura 2.4: Ídem a la Figura 2.3 para el modelo HM. 


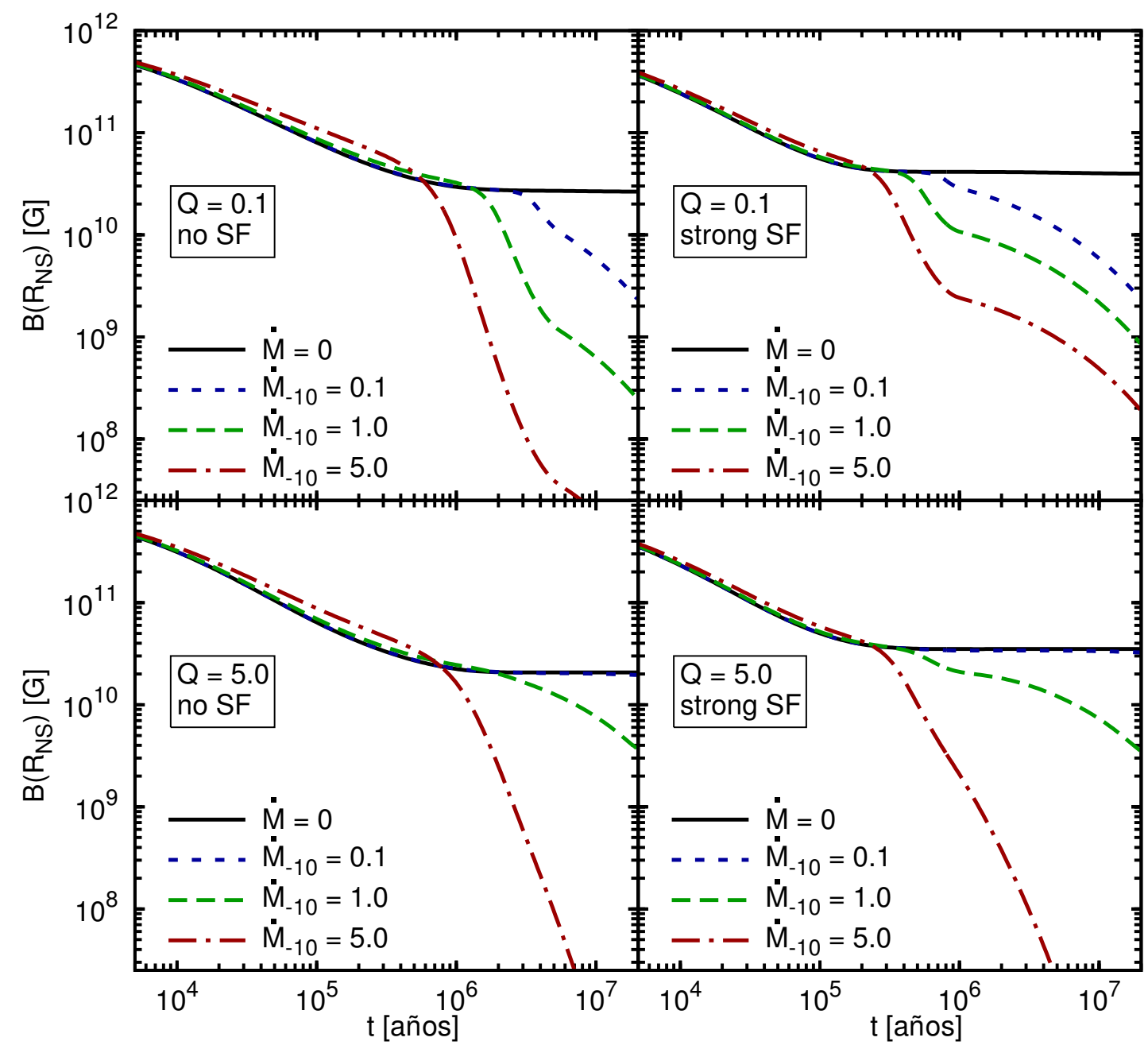

Figura 2.5: Evolución del campo magnético en la superficie para el modelo LM, para diferentes valores del parámetro de impurezas y curvas de enfriamiento.

ocurre si el proceso de acreción es apagado artificialmente (fijando $\dot{M}=0$ ). En este caso, una vez que el campo magnético alcanza la interfaz entre la corteza y el núcleo (aproximadamente a $t \sim 10^{5}-10^{6}$ años), donde la conductividad es extremadamente alta, la función de Stokes se congela en toda la corteza, y el campo magnético en la superficie permanece estable a $\sim 2 \times 10^{10} \mathrm{G}$.

En resumen, nuestros resultados muestran que el decaimiento del campo es siempre rápido en estrellas jóvenes ( $t \lesssim 10^{5}$ años) de baja masa, al menos por el tiempo que permanecen calientes, en acuerdo con resultados previos hallados por Urpin \& Muslimov (1992) y Konar \& Bhattacharya (1997). Al haber incorporado microfísica y curvas de enfriamiento actualizadas, las escalas de tiempo varían sólo levemente.

Para el modelo de mayor masa HM (Figura 2.6), la densidad en el núcleo es suficientemente alta como para encender la emisión de neutrinos a través del proceso Urca directo (ver Yakovlev 


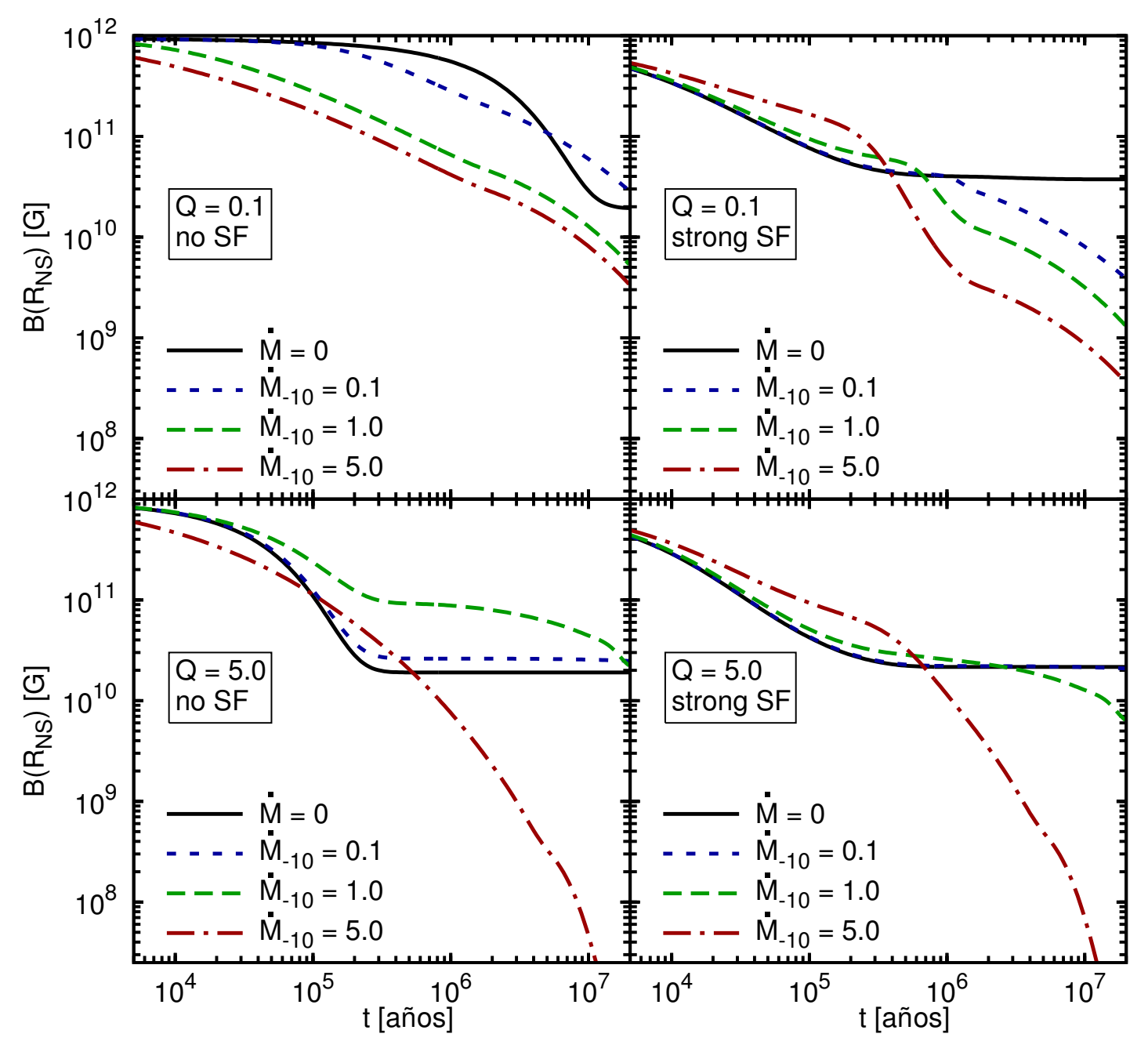

Figura 2.6: Ídem a la Figura 2.5 para el modelo HM.

et al., 2001). En el caso en que no consideramos neutrones superfluidos en el núcleo (modelo no $S F$ ), la estrella se enfría muy rápidamente y, consecuentemente, el campo magnético decae muy despacio durante la primera etapa ( $t \lesssim 10^{5}$ años). Por el contrario, en el caso en que consideramos neutrones superfluidos (en el modelo strong $S F$ ), el comportamiento de la evolución del campo magnético resulta muy similar al caso LM. En el caso HM, el parámetro de impurezas pasa a resultar crucial, ya que sólo si el contenido de impurezas es suficientemente alto $(Q=5.0)$ el campo magnético es capaz de decaer hasta $B \lesssim 10^{8} \mathrm{G}$ en $t \lesssim 10^{7}$ años, mientras que si el contenido de impurezas es bajo $(Q=0.1)$, el campo superficial permanece un factor 10 por encima de ese valor.

Hasta aquí, los resultados mostrados fueron obtenidos suponiendo que el campo magnético fue expulsado hasta la corteza externa durante la formación de la NS, fijando el parámetro $r_{\mathrm{i}}$ de la función inicial de Stokes (ver Ecuación 2.4) a la densidad ND, $\left(\rho_{\mathrm{i}}=\rho_{\mathrm{ND}}\right)$. Con el objeto de estudiar la dependencia de estos resultados con la distribución de campo magnético inicial, 


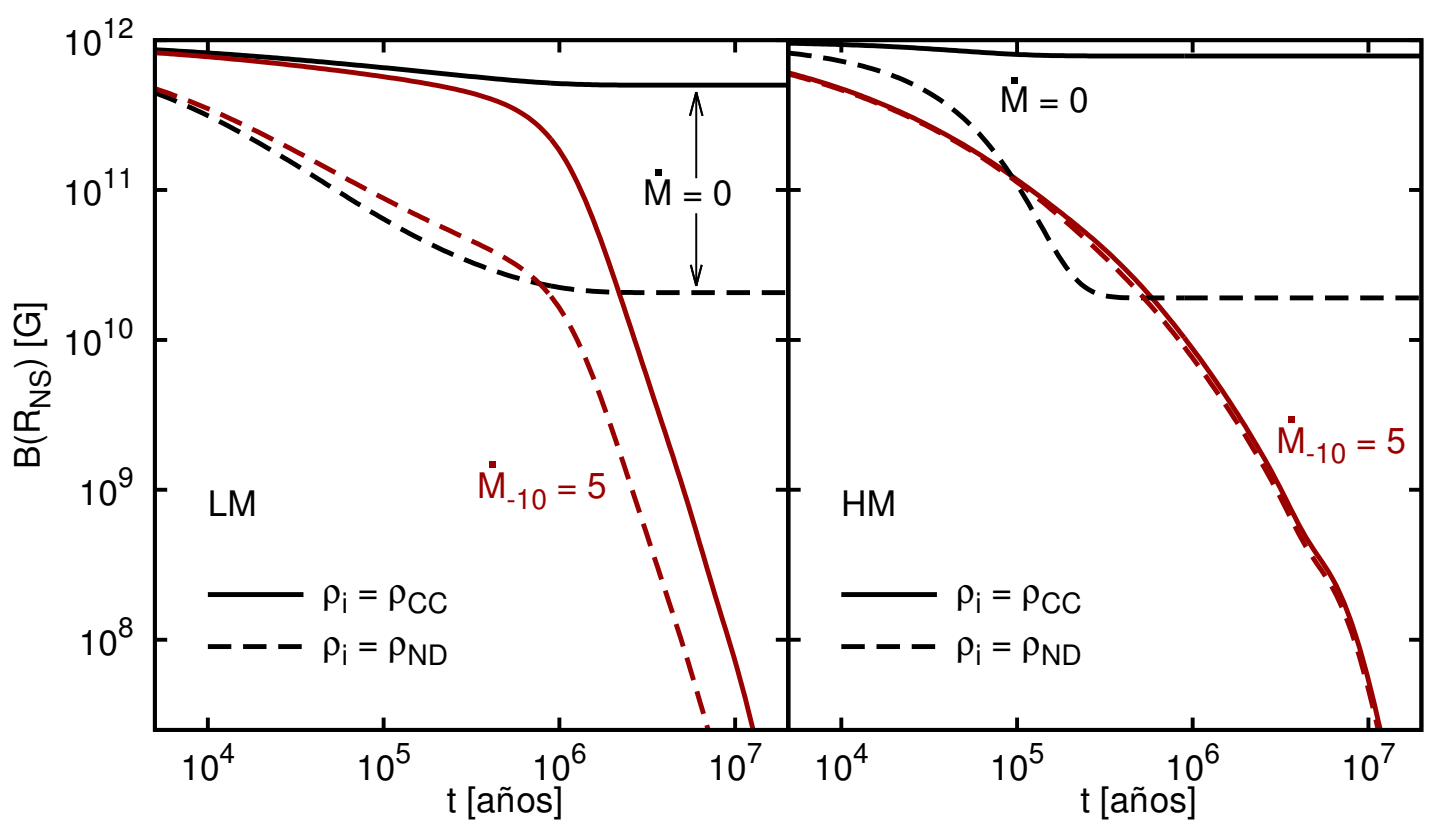

Figura 2.7: Evolución del campo magnético en la superficie para el modelo LM (panel izquierdo) y el modelo HM (panel derecho) para dos valores diferentes del parámetro de expulsión del campo magnético inicial: $\rho_{\mathrm{i}}=\rho_{\mathrm{CC}} \approx 1.3 \times 10^{14} \mathrm{gr} \mathrm{cm}^{-3}$ (líneas continuas) y $\rho_{\mathrm{i}}=\rho_{\mathrm{ND}}=4.2 \times 10^{11} \mathrm{gr} \mathrm{cm}^{-3}$ (línea de trazos). Se comparan dos escenarios extremos: $\dot{M}_{-10}=5$, para una tasa de acreción grande, y $\dot{M}_{-10}=0$, en ausencia de acreción (caso difusivo puro). En todos los casos fijamos $Q=5.0$ para el modelo no $S F$.

calculamos entonces la evolución del campo magnético considerando valores diferentes para este parámetro libre.

En la Figura 2.7 presentamos los resultados obtenidos para los modelos LM y HM, usando dos casos extremos para la distribución inicial del campo magnético. Comparamos los resultados mostrados en los paneles inferiores izquierdos de las figuras 2.5 y 2.6 obtenidos fijando $\rho_{\mathrm{i}}=\rho_{\mathrm{ND}}$ con los correspondientes a una distribución más inicial más profunda, $\rho_{\mathrm{i}}=\rho_{\mathrm{CC}} \approx 1.3 \times 10^{14} \mathrm{gr} \mathrm{cm}^{-3}$, que alcanza la interfaz entre la corteza y el núcleo. En este último caso, cuando $\dot{M}=0$, el campo magnético permanece prácticamente constante por $10^{7}$ años debido a la baja resistividad de la corteza interna. Por el contrario, para tasas de acreción altas $\left(\dot{M}=5 \times 10^{-10} \mathrm{M}_{\odot}\right.$ año $\left.{ }^{-1}\right)$ la tendencia obtenida para cualquiera de las dos distribuciones iniciales resulta muy similar.

Bajo estas hipótesis, encontramos que el campo magnético decae en escalas de tiempo que son siempre dominadas fuertemente por la tasa de acreción. En general, vemos que para $\dot{M} \gtrsim$ $10^{-10} \mathrm{M}_{\odot}$ año ${ }^{-1}$ el campo magnético puede decrecer hasta $B \lesssim 10^{8} \mathrm{G}$ en $\sim 10^{7}$ años. Además, encontramos que a estas escalas de tiempo la intensidad del campo magnético superficial depende tanto de la masa de la NS como del espesor de su corteza y del enfriamiento resultante. La evolución térmica vuelve así importante al contenido de impurezas. En este sentido, en el caso del modelo de mayor masa, HM, encontramos que el campo magnético puede reducirse hasta cuatro 
órdenes de magnitud en menos de $10^{7}$ años, considerando únicamente un alto grado de impurezas.

Es importante notar que si bien aún no hay una relación cuantitativa clara entre el parámetro de impurezas y el proceso de acreción, caracterizado por la tasa de acreción y la masa total acretada, se cree que estas cantidades deberían estar fuertemente relacionadas entre sí.

\subsection{Discusión de los resultados en el contexto de los SFXTs}

En los últimos años, gracias a los relevamientos del plano Galáctico realizados por el satélite INTEGRAL, el número de HMXBs conocidos se ha incrementado significativamente. En particular, han sido detectados varios sistemas compuestos por un objeto compacto (un agujero negro o una NS) y una estrella supergigante de gran masa (de tipo espectral O o B). Estas estrellas poseen vientos intensos con pérdidas de masa de $10^{-6} \mathrm{M}_{\odot}$ año ${ }^{-1}$ y velocidades $v_{v} \sim 1000-2000 \mathrm{~km} \mathrm{~s}^{-1}$ (Vink et al., 2000), que producen emisión de rayos X intensa debido a la acreción de este viendo por el objeto compacto. Algunos HMXBs muestran actividad debida a erupciones transitorias en rayos $\mathrm{X}$, con grandes rangos dinámicos (3-5 órdenes de magnitud) en escalas de tiempo breves (desde algunas horas hasta días) (Negueruela et al., 2006; Sguera et al., 2005). La distancia obtenida a partir de sus contrapartidas ópticas/IR implican luminosidades en los rayos $\mathrm{X}$ típicas de $L_{\mathrm{X}} \sim 10^{36} \mathrm{erg} \mathrm{s}^{-1}$ en erupción y $L_{\mathrm{X}} \sim 10^{32} \mathrm{erg} \mathrm{s}^{-1}$ en inactividad. Generalmente, estas fuentes se encuentran en un estado intermedio de $L_{\mathrm{X}} \sim 10^{33}-10^{34} \mathrm{erg} \mathrm{s}^{-1}$, producto de acreción residual sobre el objeto compacto (Sidoli et al., 2008).

En algunos de estos sistemas, los episodios de actividad son regulares con períodos coincidentes con la órbita del sistema binario, pero en la mayoría de los casos, no ha podido hallarse un período por lo cual prevalece un comportamiento azaroso. Para explicar esta fenomenología, in't Zand (2005) propusieron que las erupciones transitorias en rayos X podrían ser producidas por la acreción de grumos originados en el viendo fuertemente estructurado de la compañera supergigante (ver, por ejemplo, Owocki \& Cohen, 2006; Runacres \& Owocki, 2005). Esta idea fue explorada por Walter \& Zurita Heras (2007) y luego por Negueruela et al. (2008), dando lugar a un modelo detallado de la estructura grumosa del viento desarrollado por Ducci et al. (2009). Para explicar las erupciones periódicas de IGR J11215-5952, Sidoli et al. (2007) propusieron que la actividad regular podría generarse cuando el objeto compacto cruza una componente del viento más lenta y densa con forma de disco, dos veces por cada período orbital, en un escenario acretante. En otro modelo propuesto por Grebenev \& Sunyaev (2007) los autores sugieren que la emisión transitoria de alta energía podría producirse por la interacción del viento estelar con la magnetósfera de la NS, a través de un mecanismo de compuerta. Bozzo et al. (2008) mostraron que este fenómeno sería posible si el campo magnético de la NS fuera del orden de $B \sim 10^{14}-10^{15} \mathrm{G}$, como en los magnetares. La detección de una línea de emisión ciclotrón a 3.3 keV en el SFXT IGR J184830311 permite inferir un campo magnético de $B \sim 10^{11} \mathrm{G}$, suponiendo un origen electrónico. Sin embargo, la presencia de un magnetar no puede descartarse en este caso si la línea es producida por protones, para los cuales $B \sim 5 \times 10^{14} \mathrm{G}$ (Sguera et al., 2010).

Tres SFXTs (y un candidato) coinciden posicionalmente con fuentes de rayos $\gamma$ transitorias no identificadas, detectadas por los satélites EGRET y/o AGILE, como mencionamos arriba. Es- 
tos son IGR J20188+3647/AGL J2022+3622, AX J1841.0-0536/3EG J1837-0423, IGR J112155952/3EG J1122-5946 (Sguera, 2009), e IGR J17354-3255/AGL J1734-3310 (Sguera et al., 2011). Si bien no han sido reportadas fuentes Fermi coincidentes, esto no es contundente, debido al carácter transitorio de estos fenómenos y a la operación en modo de relevamiento de todo el cielo que realiza este instrumento ${ }^{2}$. Si la emisión de rayos $\gamma$ es real, la asociación espacial abre la posibilidad de un origen común para la emisión a diferentes longitudes de onda.

A continuación discutimos brevemente las características de cada fuente.

\section{AX J1841.0-0536}

AX J1841.0-0536 es un pulsar de rayos X transitorio $\left(P_{\mathrm{s}}=4.7 \mathrm{~s}\right)$ detectado con el satélite $A S C A$ en 1994 y 1999 (Bamba et al., 2001), cuando emitió fulguraciones de rayos $\mathrm{X}$ de un factor $\sim 10$ en escalas de tiempo de $\sim 1$ hora. A través de una observación del satélite Chandra, Halpern \& Gotthelf (2004) determinaron sus coordenadas con precisión, lo que permitió a Halpern et al. (2004) hallar su contrapartida óptica/IR, que es una estrella supergigante de tipo espectral B1 Ib (Nespoli et al., 2008). La fuente fue detectada también en actividad por INTEGRAL y Swift, y finalmente confirmada como miembro de la clase de los SFXT luego de un estudio detallado de Romano et al. (2011).

En el tercer catálogo de EGRET, 3EG J1837-0423 es una fuente puntual transitoria, y a pesar que su correlación espacial con AX J1841.0-0536 es ambigua, la ausencia de otra fuente de rayos X duros en la región sugiere una relación física entre ellas (Sguera et al., 2009).

\section{IGR J11215-5952}

Esta fuente fue descubierta por Lubiński et al. (2005) a partir de observaciones de INTEGRAL. Está asociada con una supergigante B0.7 a una distancia de $8 \mathrm{kpc}$ (Negueruela et al., 2007). Emisión pulsada con $P_{\mathrm{s}}=187$ s (Swank et al., 2007) sugiere que el objeto compacto en este SFXT es una NS. Además, esta fuente resulta ser el primer miembro con comportamiento periódico, presentando actividad regular cada 165 días de acuerdo con Romano et al. (2009), con luminosidades típicas en los rayos $\mathrm{X}$ de $L_{\mathrm{X}} \sim 5 \times 10^{36} \mathrm{erg} \mathrm{s}^{-1}$, y largos estados de quietud de $L_{\mathrm{X}} \sim 10^{33} \mathrm{erg} \mathrm{s}^{-1}$.

La fuente EGRET no identificada EGR J1122-5946 está muy bien correlacionada espacialmente con SFXT IGR J11215-5952 (Sguera, 2009). Además, la ausencia de otras contrapartidas en la banda de rayos $\gamma$ suaves abre la posibilidad de una asociación física entre ellas. Lamentablemente, las observaciones de EGRET no permiten confirmar la naturaleza constante o transitoria de dicha fuente.

\footnotetext{
${ }^{2} \mathrm{El}$ paso de detección de fuentes del último catálogo de Fermi (Nolan et al., 2012) fue aplicado únicamente al conjunto de datos completo correspondiente a una integración de 24 meses, y aún no se ha implementado en el catálogo un método de búsqueda sistemático para una fracción pequeña del intervalo de tiempo total de observación. La probabilidad de detección a priori de fuentes transitorias de brillo moderado con ciclos de actividad muy breves, tales como las esperables para los SFXTs, es muy baja.
} 


\section{IGR J20188+3647}

En 2004, la fuente de rayos X transitoria IGR J20188+3647 fue descubierta a partir de relevamientos del satélite INTEGRAL. Las propiedades de sus erupciones de rayos X, con escalas de tiempo de $\sim 1$ hora, se asemejan a las de los SFXTs, por lo cual es considerada como un candidato (Sguera et al., 2006).

Una fuente de rayos $\gamma$ no identificada, denominada AGL J2022+3622, fue observada con AGILE (Chen et al., 2007) en la misma región. Se trata de una fuente variable en la banda de los $\mathrm{MeV}$, que fuera vista en actividad por tan sólo un día. IGR J20188+3647 es la única fuente de rayos X duros en el círculo de error posicional de la fuente AGILE (Sguera, 2009).

\section{IGR J17354-3255}

IGR J17354-3255 es una fuente transitoria de rayos X duros descubierta con INTEGRAL (Kuulkers et al., 2006, 2007). Con un período orbital de 8.4 días (D'Aì et al., 2011), la fuente presenta erupciones con luminosidades de rayos $\mathrm{X} L_{\mathrm{X}} \gtrsim 10^{36} \mathrm{erg} \mathrm{s}^{-1}$ con un rango dinámico $\gtrsim 20$, típico de un SFXT intermedio (Bozzo et al., 2012; Sguera et al., 2011). En un trabajo reciente, Coleiro et al. (2013) encontraron la contrapartida en el IR cercano del sistema, una estrella supergigante 09Iab, confirmando su pertenencia a la clase de SFXTs.

Esta fuente INTEGRAL es la única fuente de rayos X duros localizada dentro del círculo de error de posición de la fuente no identificada de rayos $\gamma$ AGL J1734-3310 (Sguera et al., 2011; Sguera, 2013), que es una fuente $\mathrm{MeV} / \mathrm{GeV}$ transitoria detectada en erupción con el satélite $A G I$ LE (Bulgarelli et al., 2009). Aquí la posible asociación física se basa no sólo en la correlación espacial, sino también en su comportamiento temporal a diferentes bandas de energía. Sin embargo, basados en las observaciones en la banda de rayos X blandos del satélite Swift/XRT, Ducci et al. (2013) sugieren que IGR J17354-3255 es un HMXB prácticamente persistente. Los autores argumentaron en contra de la naturaleza SFXT de la fuente, proponiendo que su variabilidad tiene origen en eclipses del sistema binario.

Es importante remarcar que la única evidencia observacional disponible para sostener una posible asociación física entre estas fuentes es una correlación espacial, y a veces temporal. Es evidente que son necesarias observaciones de larga exposición tanto en la banda rayos X como en los rayos $\gamma$ para establecer si esta conexión física es real.

En un trabajo reciente, Sguera et al. (2009) introdujeron un escenario para explicar la producción de partículas relativistas capaces de generar una fuente transitoria de rayos $\gamma$, bajo las condiciones particulares impuestas por los SFXTs, basado en la formación de jets temporarios en una torre magnética. Tal aceleración de partículas podría expulsar una fracción considerable del material acretante (Kato, 2007). En el modelo magneto-centrífugo de formación de jets, la eyección de materia es posible únicamente si el material de la región interna del disco de acreción puede alcanzar distancias menores a $\sim 40$ radios gravitacionales (Kato et al., 2004). Para ello, el radio de Alfvén, $R_{\mathrm{A}}$, no puede ser mayor que el radio de la estrella, $R_{\mathrm{NS}}$, por lo cual el campo magnético de la NS debe ser suficientemente débil como para permitir la penetración del material. Siguiendo este razonamiento, Massi \& Kaufman Bernadó (2008)derivaron una condición básica 
para formación de jets en NSs acretantes, formulando que la intensidad del campo magnético en la superficie de la NS debe ser $B \lesssim 10^{8} \mathrm{G}$.

A pesar que la emisión de jets relativistas pudo ser observada en algunos LMXBs (por ejemplo, en Cir X-1, Fender et al., 1998), este fenómeno aún no ha sido detectado en HMXBs (ver la discusión acerca de LS I +61 303 Romero et al. (2007), fuente para la cual han sido propuestos modelos de emisión de jets, Bosch-Ramon et al. (2006)). Nuestros modelos numéricos sugieren que el campo magnético de una NS puede decaer de una intensidad típica en su superficie de $B=10^{12} \mathrm{G}$ hasta $B \lesssim 10^{8} \mathrm{G}$ en escalas de tiempo de $t \sim 10^{7}$ años, permitiendo la formación de jets, siempre que una fracción significativa del viento intenso emitido por la estrella de gran masa del sistema sea acretada por la NS.

En la Figura 2.8 resumimos la escala de tiempo de decaimiento de campo magnético desde un valor típico de un pulsar joven, de $B=10^{12} \mathrm{G}$ hasta $B \lesssim 10^{8} \mathrm{G}$, necesario para satisfacer la condición de formación de jets, como función del contenido de impurezas de la corteza, $Q$, y la tasa de acreción, $\dot{M}$, para los modelos LM y HM (considerando ausencia de superfluidez de los neutrones en el núcleo, modelo no $S F$ ). En el gráfico, puede verse que si $\dot{M}<6 \times 10^{-11} \mathrm{M}_{\odot}$ año ${ }^{-1}$, el campo magnético no puede decaer cuatro órdenes de magnitud en $t<2 \times 10^{7}$ años. Por el contrario, si $\dot{M}>1.7 \times 10^{-10} \mathrm{M}_{\odot}$ año ${ }^{-1}$, el campo magnético podría decaer más de cuatro órdenes de magnitud en la superficie de la NS para $Q>0.65$ en el modelo HM, mientras que en el modelo LM, tal decaimiento podría ser posible para todos los valores del contenido de impurezas explorados. En ambos modelos, para $0.6 \lesssim Q \lesssim 1.2$, un decaimiento de cuatro órdenes de magnitud podría ser posible para un rango de valores de $\dot{M}$ mucho más amplio.

En nuestro modelo, hemos supuesto una serie de hipótesis cuyas limitaciones se discuten a continuación. Primero, el campo magnético posee una geometría fija: configuración dipolar. Hemos despreciado términos de mayor orden que no permitimos evolucionar. Sin embargo, la reorganización local del campo ha mostrado sólo tener efectos importantes en escalas de tiempo cortas y en fuentes fuertemente magnetizadas como los magnetares con $B \sim 10^{14} \mathrm{G}$ (Pons et al., 2009; Viganò et al., 2012, 2013). A pesar de que la inclusión de términos de mayor orden e la descripción del campo magnético puede resultar más realista, la evolución de largo término debería ser dominada por la geometría global del campo, por su difusión óhmica y por la convección o advección sufrida dada la acreción de materia.

En segundo lugar, supusimos un proceso de acreción esférico y con tasa de acreción constante, ignorando la desviación de material a través de los polos magnéticos de la estrella así como la dinámica del viento estelar a lo largo de $\sim 10^{7}$ años. Para campos magnéticos de $B \sim 10^{8}-10^{12} \mathrm{G}$ y tasas de acreción de viento típicas, la densidad de energía magnética cerca de la NS es mayor a la densidad de energía del material acretado, por lo que la materia será direccionada hacia la superficie de la NS a través de canales angostos cerca de sus polos. Un escenario más realista debería incluir un tratamiento bidimensional en el cual el material acretado es acumulado en los polos magnéticos con una tasa de acreción local dependiente de la evolución de la estrella donante. Si bien la modificación de los resultados presentados aquí, bajo un tratamiento bidimensional para la acreción columnar es difícil de predecir, cualitativamente podemos afirmar que el enterramiento del campo magnético que domina la evolución del campo magnético en la superficie, 


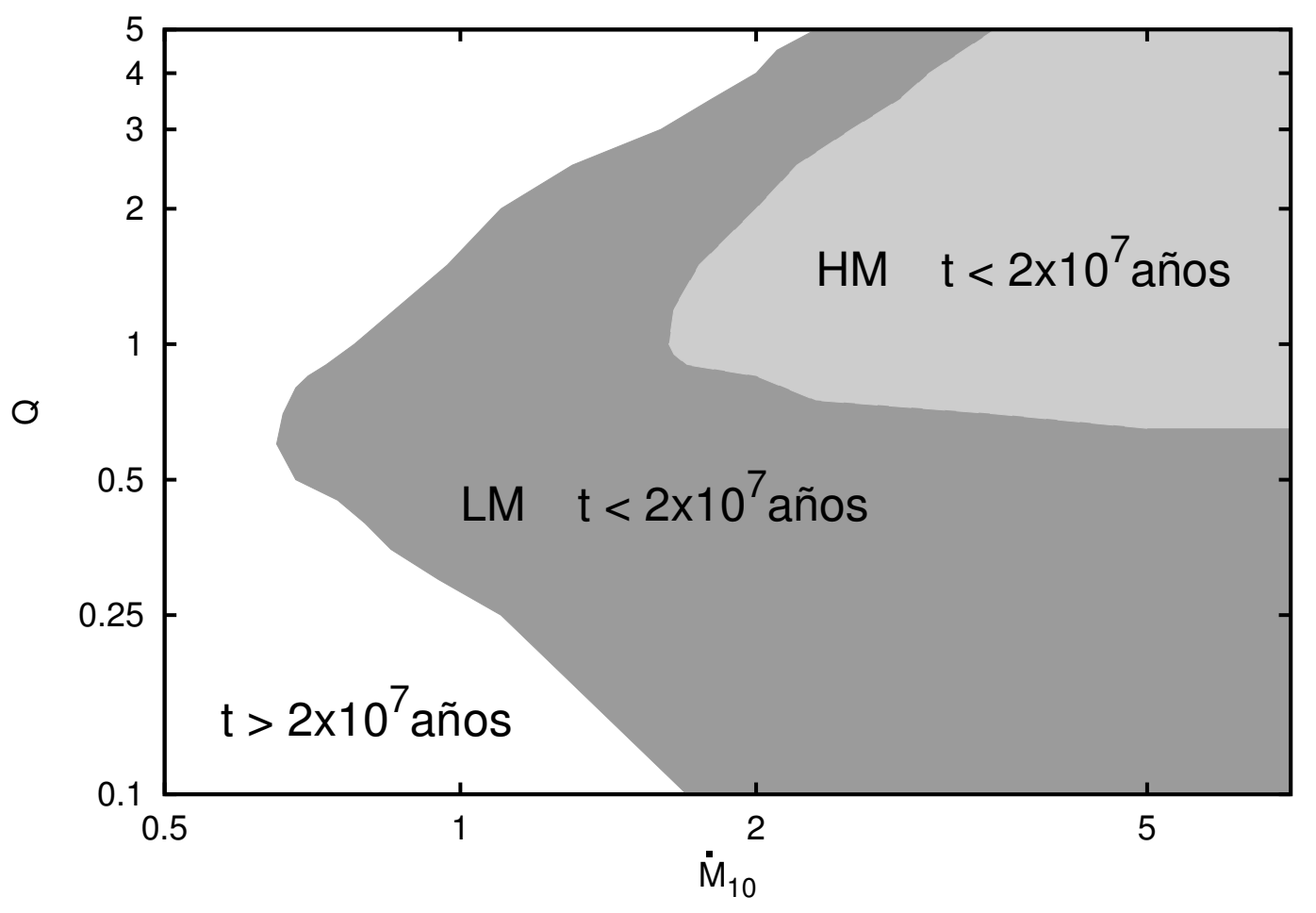

Figura 2.8: Escala temporal, $t$, para el decaimiento del campo magnético superficial desde $10^{12} \mathrm{G}$ hasta $\sim 10^{8} \mathrm{G}$, como función del contenido de impurezas, $Q$, y la tasa de acreción, $\dot{M}_{-10}$, en unidades de $10^{-10} \mathrm{M}_{\odot}$ año ${ }^{-1}$, para los modelos LM y HM, en el caso no $S F$.

debería llevarse adelante también en el caso más complejo, por lo que el efecto neto encontrado en el tratamiento unidimensional con simetría esférica podría jugar también un papel importante en el escenario de acreción columnar propuesto (ver, por ejemplo, Lovelace et al., 2005; Payne \& Melatos, 2004, 2007). Los resultados mostrados en este trabajo motivan la realización de estudios futuros más profundos en esta dirección, pero destacamos aquí que tales esfuerzos teóricos pueden no estar en correspondencia con otras incertezas presentes, tales como las tasas de acreción inferidas de las observaciones o la dinámica estelar propia de la estrella compañera y su viento.

Otra aproximación adoptadas radica en la hipótesis de corteza isotérmica y que la evolución térmica no considera la posible deposición de calor provocada por el proceso de acreción. Sin embargo, en la evolución de largo plazo, los resultados parecen ser mayormente afectados por la composición de la corteza, por lo que hemos considerado aquí una EoS modificada por acreción, consistente con la corteza asumida para el cálculo de los perfiles de evolución térmica de Aguilera et al. (2008).

Finalmente, el contenido de impurezas ha sido fijado como un parámetro constante en toda la corteza externa, de manera independiente a la intensidad del proceso de acreción (ya sea por la masa total acretada y/o la tasa de acreción y su duración) ya que no se ha establecido aún 
una relación cuantitativa entre estas cantidades. Estudios recientes de la emisión térmica de NS en LMXBs en estado de quietud, indican la necesidad de imponer valores $Q \sim 1$ para ajustar los datos de dos fuentes cuyas tasas de acreción están en el rango de $1-5 \times 10^{-10} \mathrm{M}_{\odot}$ año ${ }^{-1}$ (Turlione et al., 2015).

\subsection{Conclusiones}

En este trabajo estudiamos condiciones necesarias para la formación de jets en sistemas binarios formados por una NS que sobrelleva un decaimiento de su campo magnético inducido por la acreción del viento generado por una estrella compañera de gran masa. Usamos un modelo numérico propio que incorpora el estado del arte en la microfísica y perfiles de evolución térmica obtenidos de forma consistente (Aguilera et al., 2008). Nuestro tratamiento tuvo en cuenta la difusión de las corrientes eléctricas en la corteza de una NS y la advección del campo magnético provocada por la acreción de materia desde su superficie. Una fuerte limitación de nuestro modelo radica en que considera sólo acreción esféricamente simétrica en vez de acreción columnar. Además, elegimos focalizarnos en los aspectos más cualitativos que rigen la evolución del campo magnético en su superficie, tales como el enterramiento del campo magnético, que se espera jueguen un papel importante en el área sometida a la acreción directa en el caso de un tratamiento bidimensional, que podrían a su vez resultar fundamentales para la posibilidad de que jets relativistas puedan ser emitidos en este contexto.

Los resultados indican que la escala de tiempo para el decaimiento del campo magnético depende fuertemente de la tasa de acreción, siendo menos sensible al parámetro de impurezas, $Q$, e indirectamente, a través del perfil de enfriamiento, a la masa de la NS y del estado de superfluidez de los neutrones del núcleo. En el caso que una fracción significativa del viento emitido por la estrella compañera sea acretado por la NS, dando lugar, por ejemplo, a tasas de acreción $\dot{M} \gtrsim 10^{-10} \mathrm{M}_{\odot}$ año ${ }^{-1}$, el campo magnético podrá decaer desde un valor típico inicial de $B=10^{12} \mathrm{G}$ hasta $B \lesssim 10^{8} \mathrm{G}$ en una escala temporal de $t \sim 10^{7}$ años. Por lo tanto, debería ser posible que NS sean capaces de formar jets en HMXBs.

Nuestros resultados son importantes para modelos de SFXTs y binarias de rayos $\gamma$ tales como LS I +61 303 o LS 5039, donde han sido observados fenómenos de emisión no térmica y han sido deducidas tasas de acreción de $\sim 10^{-10} \mathrm{M}_{\odot}$ año ${ }^{-1}$ a partir de simulaciones numéricas (Owocki et al., 2011; Romero et al., 2007). En particular, los fenómenos transitorios de emisión rápida de rayos X podrían convertirse así en una nueva clase de fuentes $\mathrm{MeV}$ de la Galaxia, como fue sugerido por Sguera et al. (2009) para SFXT AX J1841.0-0536.

Resultan necesarias observaciones profundas de las cuatro fuentes candidatas presentadas aquí, en la banda de los rayos $\gamma$, con los satélites Fermi y AGILE para poder confirmar la existencia de la inyección de partículas relativistas temporales cuando, por ejemplo, son acretados grandes grumos provenientes del viento estelar, posibilitando la eyección. Por otro lado, observaciones dedicadas a más altas energías usando MAGIC II y HESS II podrían resultar importantes ya que permitirían establecer cotas al nivel de radiación no térmica durante las fulguraciones de rayos X y para determinar la energía máxima de corte de las partículas relativistas. Observaciones 
interferométricas en radio con gran resolución angular serán necesarias para determinar si estas eyecciones relativistas son reales. La detección de un jet transitorio en un HMXB que albergue una NS, podría proveer evidencia indirecta de nuestras predicciones para el decaimiento del campo magnético. 


\section{Capítulo 3}

\section{Un modelo simple para el frenado o anti-glitch observado en el magnetar $1 \mathrm{E} 2259+586$}

\subsection{Resumen}

En este capítulo presentamos un modelo simple propuesto como un mecanismo interno capaz de explicar el frenado o anti-glitch recientemente observado en el magnetar AXP 1E 2259+586, basado en la idea de que el decaimiento acumulado de la componente toroidal del campo magnético interno de este magnetar podría volverse suficiente para volver inestable una configuración alargada inicialmente estable de su estructura. En ese caso, el re-acomodamiento posterior de la corteza y el interior de la estrella resultarían así en un frenado repentino en su velocidad de rotación.

Mostramos cálculos de ordenes de magnitud que dan viabilidad a este escenario, utilizando un modelo estelar analítico simple, pero que resulta una hipótesis razonable para una primera aproximación. Estimamos además que la energía liberada por el mecanismo propuesto está en acuerdo con las observaciones.

Basados en este modelo, predecimos que se requiere un campo magnético de al menos unos $10^{14} \mathrm{G}$ para poder producir el frenado repentino observado en el anti-glitch, que resulta un valor típico para los magnetares, mientras que, consecuentemente, este tipo de actividad no resulta esperable en pulsares normales, donde los campos magnéticos son al menos dos órdenes de magnitud menos intensos.

\subsection{Introducción}

Los magnetares son NSs alimentadas por sus campos magnéticos internos extremadamente intensos (Duncan \& Thompson, 1992). El estudio detallado de la evolución temporal de la emisión producida por los magnetares puede ser útil para poner cotas tanto a los valores de la 
intensidad de su campo magnético dipolar externo como a su estructura interior (Chamel et al., 2008; Kouveliotou et al., 1998). A pesar de que todas las NSs sufren un lento frenado a grandes escalas temporales, debido al decaimiento de su campo magnético, muchas de ellas sufren leves aceleraciones repentinas, conocidas como anomalías o glitches en la literatura (Espinoza et al., 2011; Yu et al., 2013). En un trabajo reciente (Archibald et al., 2013) fue presentada evidencia clara de la detección del primer frenado repentino de uno de estos objetos, que fue observado en el magnetar AXP 1E 2259+586, y llamado anti-glitch. Allí los autores proponen dos posibles interpretaciones: (i) un anti-glitch seguido por un glitch normal ( 100 días después) y (ii) una secuencia de dos anti-glitches (separados $\sim 50$ días). Si bien otros eventos de frenado fueron observados en los magnetares SGR 1900+14 (Woods et al., 1999) y 4U 0142+61 (Gavriil et al., 2011), y el pulsar de gran campo magnético PSR J1846-0258 (Livingstone et al., 2010), ninguno de ellos es considerado estrictamente como un anti-glitch (repentino), debido a las grandes escalas de tiempo involucradas (17 - 127 días).

El pulsar anómalo de rayos X AXP 1E 2259+586 tiene un período de $\sim 7 \mathrm{~s}$, edad característica de $\sim 10^{6}$ años y un campo magnético dipolar inferido de $B_{d} \sim 5.9 \times 10^{13} \mathrm{G}^{1}$, que impone un valor mínimo a la intensidad de su campo magnético interno. AXP 1E 2259+586 ha sido monitoreado por Rossi X-ray Timing Explorer y Swift X-ray Telescope durante las últimas dos décadas, presentando un frenado estable, con la excepción de dos glitches observados en 2002 (Kaspi et al., 2003) y 2007 (Içdem et al., 2012), un evento en 2009 (Içdem et al., 2012) y el anti-glitch aquí analizado, en 2012.

Uno de los hechos observacionales que deben ser ajustados por cualquier modelo propuesto para explicar este anti-glitch es un cambio neto en la frecuencia de rotación de $\sim-5 \times 10^{-7} \mathrm{~Hz}$ en un lapso inferior a 100 días (Archibald et al., 2013). Este frenado puede interpretarse como resultado de dos eventos repentinos de $\left(|\Delta v / v| \gtrsim 10^{-7}\right)$. Para ajustar los datos temporales obtenidos con Swift, en (Archibald et al., 2013) los autores proponen dos posibilidades: (i) un frenado en el que $\Delta v / v=-3.1(4) \times 10^{-7}$ seguido por una aceleración de $\Delta v / v=2.6(5) \times 10^{-7}$; (ii) un frenado en $\Delta v / v=-6.3(7) \times 10^{-7}$ seguido por un segundo frenado de $\Delta v / v=-4.8(5) \times 10^{-7}$. Es notable que el modelo (ii) es estadísticamente favorable por un análisis bayesiano (Hu et al., 2014).

El segundo aspecto que debe ser abordado es el energético. El 21 de abril de 2012, el telescopio Fermi/GBM detectó una erupción de rayos X duros con una duración de $36 \mathrm{~ms}$ (Foley et al., 2012) ocurrida durante el lapso asociado al anti-glitch. La energía asociada en la banda de 10$1000 \mathrm{keV}$ corresponde a una emisión isotrópica de $E_{\gamma} \sim 10^{38} \mathrm{erg}$. Además, las observaciones de Swift mostraron un incremento del flujo en los 2-10 keV en un factor 2 (Archibald et al., 2013), correspondientes a una emisión isotrópica de $E_{\mathrm{X}} \sim 10^{41} \mathrm{erg}$ (Huang \& Geng, 2014).

Una serie de explicaciones posibles para este fenómeno han sido propuestas, basadas tanto en un origen externo (Huang \& Geng, 2014; Lyutikov, 2013; Pons et al., 2009; Tong, 2014) como interno (Duncan, 2013). A pesar de las búsquedas profundas realizadas tanto en frecuencias de radio como en los rayos $\mathrm{X}$, no ha sido posible detectar radiación residual circundante posterior al evento (Archibald et al., 2013), lo cual se contrapone a un escenario basado en un origen

\footnotetext{
${ }^{1}$ http://www.physics.mcgill.ca/ ${ }^{\sim p u l s a r / m a g n e t a r / m a i n . h t m l ~}$
} 
debido al viento de partículas del pulsar. En este sentido, resulta favorecida una explicación basada en un reacomodamiento de las capas interiores de la estrella. En este trabajo proponemos un mecanismo simple para explicar el origen del frenado repentino observado. Sugerimos que como consecuencia del decaimiento natural de largo término del campo magnético interno de la estrella, una configuración inicialmente alargada (en la dirección del eje de rotación) se tornaría suficientemente inestable para romper la estructura cristalizada de la corteza de la NS. Luego, el reacomodamiento de la estructura estelar en una configuración estable más esférica, daría como resultado un frenado repentino como el anti-glitch observado. Un escenario similar fue sugerido para explicar el evento ocurrido en el magnetar SGR 1900+14 (Ioka, 2001).

Estrellas compactas deformadas por un campo magnético intenso fueron estudiadas inicialmente por Katz (1989); Ostriker \& Hartwick (1968); Wentzel (1961). Más adelante, Cutler (2002) y Haskell et al. (2008) desarrollaron un formalismo para modelar la deformación debida al campo magnético de manera más realista. En sus trabajos, las deformaciones fueron calculadas para estrellas rotantes de densidad uniforme, con combinaciones de campos magnéticos poloidales y toroidales. Un estudio similar, incluyendo EoSs más realistas fue desarrollado recientemente por Frieben \& Rezzolla (2012), quienes obtuvieron distorsiones cuadrupolares del mismo orden. Por esta razón, optamos por utilizar una estrella de densidad uniforme que posibilita realizar cálculos analíticos. En el caso de los magnetares, como AXP 1E 2259+586, las deformaciones producidas por efecto de la rotación son completamente irrelevantes, debido a sus largos períodos ( $10 \mathrm{~s})$. En estos casos, el resultado principal es que mientras que en el caso de que el campo magnético sea dominado por la componente poloidal, así como por rotación rápida, las estrellas tienden a volverse ensanchadas en el ecuador para permanecer mecánicamente estables, mientras que cuando el campo magnético es dominado por la componente toroidal, las estrellas tienden a volverse alargadas.

Para una mezcla de campo magnético toroidal y poloidal, la distorsión cuadrupolar asociada al modo $l=2$ de volumen constante, para estrellas incompresibles de densidad uniforme, viene dada por:

$$
\epsilon=\frac{I_{z z}-I_{x x}}{I_{z z}}=-\frac{25 R^{4}}{24 G_{N} M^{2}}\left(\left\langle B_{t}^{2}\right\rangle-\frac{21}{10}\left\langle B_{p}^{2}\right\rangle\right),
$$

donde $\left\langle B_{t}^{2}\right\rangle$ es el valor medio del cuadrado de la intensidad del campo magnético toroidal, $\left\langle B_{p}^{2}\right\rangle$ es el valor medio de la componente poloidal, $R$ es el radio de la estrella no deformada, $G_{N}$ es la constante gravitacional y $M$ la masa de la NS.

A pesar que un campo magnético puramente toroidal es inestable (Braithwaite, 2009), una componente poloidal adicional con energía $E_{p} / E_{t}=B_{p}^{2} / B_{t}^{2} \sim 1-5 \%$ es capaz de estabilizar la configuración de campo magnético (Reisenegger, 2013), lo que nos permite ignorar la contribución poloidal a la Ecuación 3.1 ya que $\left\langle B_{p}^{2}\right\rangle \ll\left\langle B_{t}^{2}\right\rangle$.

Por otra parte, Horowitz \& Kadau (2009) encontraron que la corteza de una NS es capaz de soportar deformaciones elípticas hasta cierto valor crítico $\epsilon_{c} \lesssim 4 \times 10^{-6}$, antes de quebrarse. 


\subsection{El modelo}

El escenario teórico que queremos explorar es el siguiente: dada una cierta configuración "inicial" de campo magnético, predominantemente toroidal caracterizada por $\left\langle B_{t}^{i}\right\rangle$, la NS se cristaliza entonces en una configuración de equilibrio alargada, con elipticidad $\epsilon^{-}$dada por la Ecuación 3.1. Luego, el campo magnético interno de la estrella decaerá por escalas de tiempo $\sim 10^{5}$ años (ver, por ejemplo, Pons et al., 2009; Viganò et al., 2013, y los resultados del Capítulo 2 de esta tesis, para tasas de acreción $\dot{M}=0$, que corresponden a estrellas aisladas) debido a los efectos Hall y Ohm sufridos por las corrientes que circulan por su corteza. Como consecuencia, esta configuración alargada con $\epsilon^{-}$se irá apartando más y más del equilibrio hasta alcanzar un esfuerzo crítico, volviéndose suficientemente inestable como para quebrar la corteza estelar. En ese momento, la estrella deberá adquirir una nueva forma, en una configuración nuevamente estable que deberá resultar menos alargada, y por ende, más esférica, con elipticidad $\epsilon^{+}$, asociada a la intensidad de campo magnético actual, que llamamos "final", $\left\langle B_{t}^{f}\right\rangle$. Así, como la configuración más esférica, $\epsilon^{+}$, posee un mayor momento de inercia respecto al eje de rotación, que fijamos como $z$ en nuestro caso, respecto a la configuración $\epsilon^{-}$, y considerando la ausencia de un torque externo, el momento angular debe conservarse, por lo que este repentino cambio en la estructura estelar da como resultado un frenado en la velocidad de rotación de la estrella, como el observado.

El cambio en el alargamiento de la estrella de densidad uniforme induce un desplazamiento en la frecuencia de rotación dado por:

$$
\frac{\Delta v}{v} \equiv \frac{v^{+}-v^{-}}{v^{-}}=\frac{I_{z z}^{-}}{I_{z z}^{+}}-1=\left(\frac{R_{e}^{-}}{R_{e}^{+}}\right)^{2}-1,
$$

donde $R_{e}^{\mp}$ y $v^{\mp}$ son los radios ecuatoriales y las frecuencias de rotación antes y después del antiglitch. En este caso, $R_{e}$ está relacionado con la elipticidad de la estrella como:

$$
R_{e}=R(1-2 \epsilon)^{-1 / 6} .
$$

Por lo tanto, podemos escribir la Ecuación 3.2 como función de las distorsiones cuadrupolares antes y después del anti-glitch, $\epsilon^{\mp}$ :

$$
\frac{\Delta v}{v}=\frac{\left(1-2 \epsilon^{+}\right)^{1 / 3}}{\left(1-2 \epsilon^{-}\right)^{1 / 3}}-1 \approx \frac{2}{3}\left(\epsilon^{-}-\epsilon^{+}\right),
$$

donde, en la última aproximación, usamos el hecho que, en los casos como los que nos interesan aquí, ambos $\left|\epsilon^{\mp}\right| \approx 10^{-6} \ll 1$.

Reemplazando la Ecuación 3.1 en la Ecuación 3.4, y suponiendo que el campo magnético está alineado con el eje de rotación de la estrella, obtenemos $\Delta v / v$ como función de $\left\langle B_{t}^{i, f}\right\rangle^{2}$ :

$$
\frac{\Delta v}{v} \approx \frac{2}{3} \frac{25 R^{4}}{24 G_{N} M^{2}}\left(\left\langle B_{t}^{f}\right\rangle^{2}-\left\langle B_{t}^{i}\right\rangle^{2}\right)
$$

de donde sigue

$$
\left\langle B_{t}^{f}\right\rangle \approx \sqrt{\left\langle B_{t}^{i}\right\rangle^{2}-B_{0}^{2}}
$$


donde $B_{0}^{2}=-\left(8.7 \times 10^{14} \mathrm{G}\right)^{2}\left(\frac{\Delta v}{v}\right)_{6}\left(\frac{M}{1.4 \mathrm{M}_{\odot}}\right)^{2}\left(\frac{R}{10 \mathrm{~km}}\right)^{-4}$ cambiaría en caso de un tratamiento más completo. Aquí $\left(\frac{\Delta v}{v}\right)_{6}=\left(\frac{\Delta v}{v}\right) / 10^{-6}$ y $\left(\frac{\Delta v}{v}\right)<0$ para anti-glitches.

Es importante notar que resultaría valioso desarrollar aquí un modelo más detallado que considere a una NS compuesta por una corteza sólida rodeando un núcleo líquido, como el propuesto por Franco et al. (2000) para estudiar terremotos estelares en pulsares alimentados por rotación. Sin embargo, resaltamos acá que en este trabajo presentamos cálculos de orden de magnitud para dar un piso de confianza al escenario que estamos proponiendo, manteniendo la analiticidad y simpleza del modelo.

Para estimar la energía emitida por este mecanismo durante el anti-glitch, usamos el modelo desarrollado por Baym \& Pines (1971) para los glitches en pulsares. En este trabajo clásico, los autores consideran tres contribuciones: energía gravitacional, proveniente del cambio global de la forma de le estrella, rotacional, asociado al desplazamiento de la frecuencia de rotación, $\Delta v / v$, y las tensiones internas liberadas por la corteza durante el evento. Esta energía es acumulada en tensiones debido a que, a pesar de que el campo magnético decae desde $\left\langle B_{t}^{i}\right\rangle^{2}$ a $\left\langle B_{t}^{f}\right\rangle^{2}$ por $10^{5}$ años, la corteza cristalizada sostiene su forma original, caracterizada por $\epsilon^{-}$incrementando su tensión interna, y alejándose así de la configuración de equilibrio. Así, una vez que la deformación crítica es alcanzada, la corteza se quiebra y la estrella se re-acomoda en una nueva configuración con elipticidad $\epsilon^{+}$liberando esta energía almacenada. Además, como estamos tratando con un magnetar y no con un pulsar corriente, debemos considerar la liberación de energía magnética debido al desplazamiento de las líneas de campo fijas a la corteza. Sin embargo, como la longitud de escala, $\ell$, asociada al cambio de forma en nuestro modelo es muy pequeña $\ell / R \sim \epsilon$, supondremos que no hay reconexión magnética, como ocurre en la teoría estándar de los repetidores de rayos $\gamma$ (SGRs; Thompson \& Duncan, 1995, 1996).

\subsection{Resultados}

Siguiendo entonces el modelo propuesto, para una NS de densidad constante, alargada por la presencia de un campo magnético predominantemente toroidal (Cutler, 2002), calculamos el decaimiento de campo magnético $\Delta\left\langle B_{t}\right\rangle$ necesario para producir el cambio de frecuencia $\Delta v / v$ observado en el anti-glitch de AXP 1E 2259+586.

En la Figura 3.1 presentamos las soluciones físicamente posibles, $\Delta\left\langle B_{t}\right\rangle<0$, a la Ecuación 3.4, como función de la intensidad media de campo magnético toroidal $\left\langle B_{t}^{i}\right\rangle$, para tres configuraciones de NSs diferentes, suponiendo un salto en la frecuencia $\Delta v / v=-6.3 \times 10^{-7}$ igual al primero de los eventos del modelo (ii).

Para una NS típica de $R=10 \mathrm{~km}$ y masa $M=1.4 \mathrm{M}_{\odot}$, con un campo toroidal medio de $\left\langle B_{t}\right\rangle=2 \times 10^{15} \mathrm{G}$, que corresponde a un valor máximo $B_{M} \gtrsim 10^{16} \mathrm{G}$ (Reisenegger, 2013) en su interior, estimamos que un decaimiento en el campo magnético de alrededor de un $\sim 10 \%$ podría explicar el frenado observado. Este resultado cualitativo resulta prácticamente independiente de otros valores aceptables para la masa, el radio y la intensidad del campo magnético de los magnetar (ver Figura 3.1). Estudios detallados de la evolución del campo magnético en NSs muestran que 


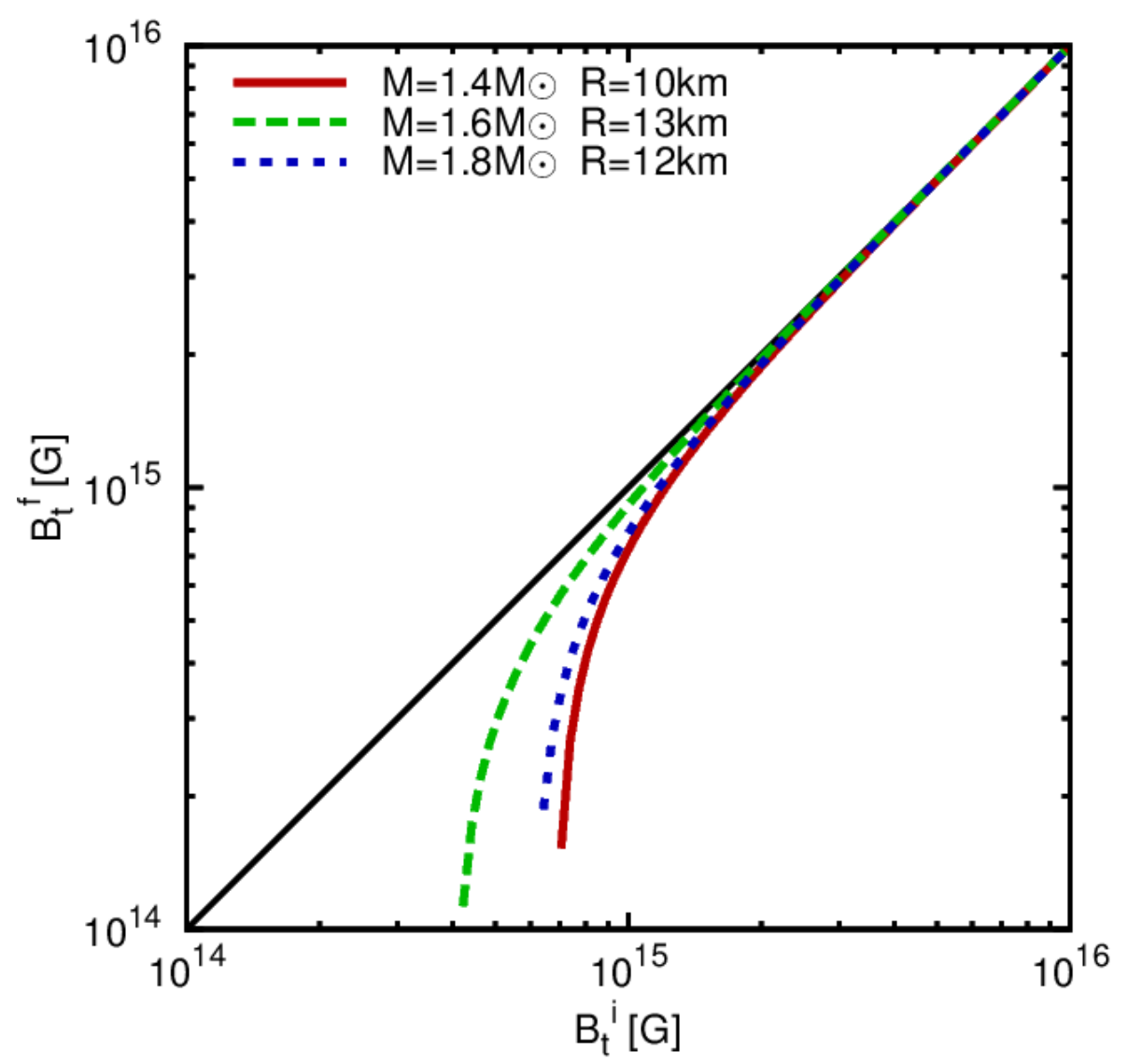

Figura 3.1: Soluciones físicamente posibles de la Ecuación 3.6 para $\Delta v / v=-6.3 \times 10^{-7}$ como función de $\left\langle B_{t}^{i}\right\rangle$, para tres configuraciones diferentes de NSs (ver la leyenda). En gris mostramos la función identidad como referencia.

un decaimiento del orden del $\sim 10 \%$ puede ser alcanzado fácilmente luego de $t \lesssim 10^{6}$ años en un magnetar como AXP 1E 2259+586 (Viganò et al., 2013).

Para cada una de las configuraciones de NSs elegidas, encontramos un valor mínimo para el campo magnético inicial, $\left\langle B_{t}^{i}\right\rangle$, necesario para encontrar una solución física a la Ecuación 3.4 dado el $\Delta v / v$ observado. Este valor crítico resulta, en cualquier caso, varias veces superior a $10^{14} \mathrm{G}$, lo que impide la posibilidad de que fenómenos de esta naturaleza puedan ocurrir también en los pulsares corrientes, permitiendo únicamente su aparición en NSs fuertemente magnetizadas, como los magnetares. Este resultado explica naturalmente porqué a pesar de que los pulsares han sido monitoreados intensamente durante varias décadas, ningún frenado repentino de estas características fue detectado aún.

En la Figura 3.2, presentamos el cambio de frecuencia $\Delta v / v$ de la Ecuación 3.4 como función del cambio en la intensidad media del campo magnético toroidal, $\left|\Delta\left\langle B_{t}\right\rangle\right| /\left\langle B_{t}^{i}\right\rangle$. Los rectángulos grises representan los valores de $\Delta v / v$ del modelo (ii) con sus barras de error correspondientes.

De esta manera, mostramos como nuestro modelo simple puede explicar el cambio repentino de 


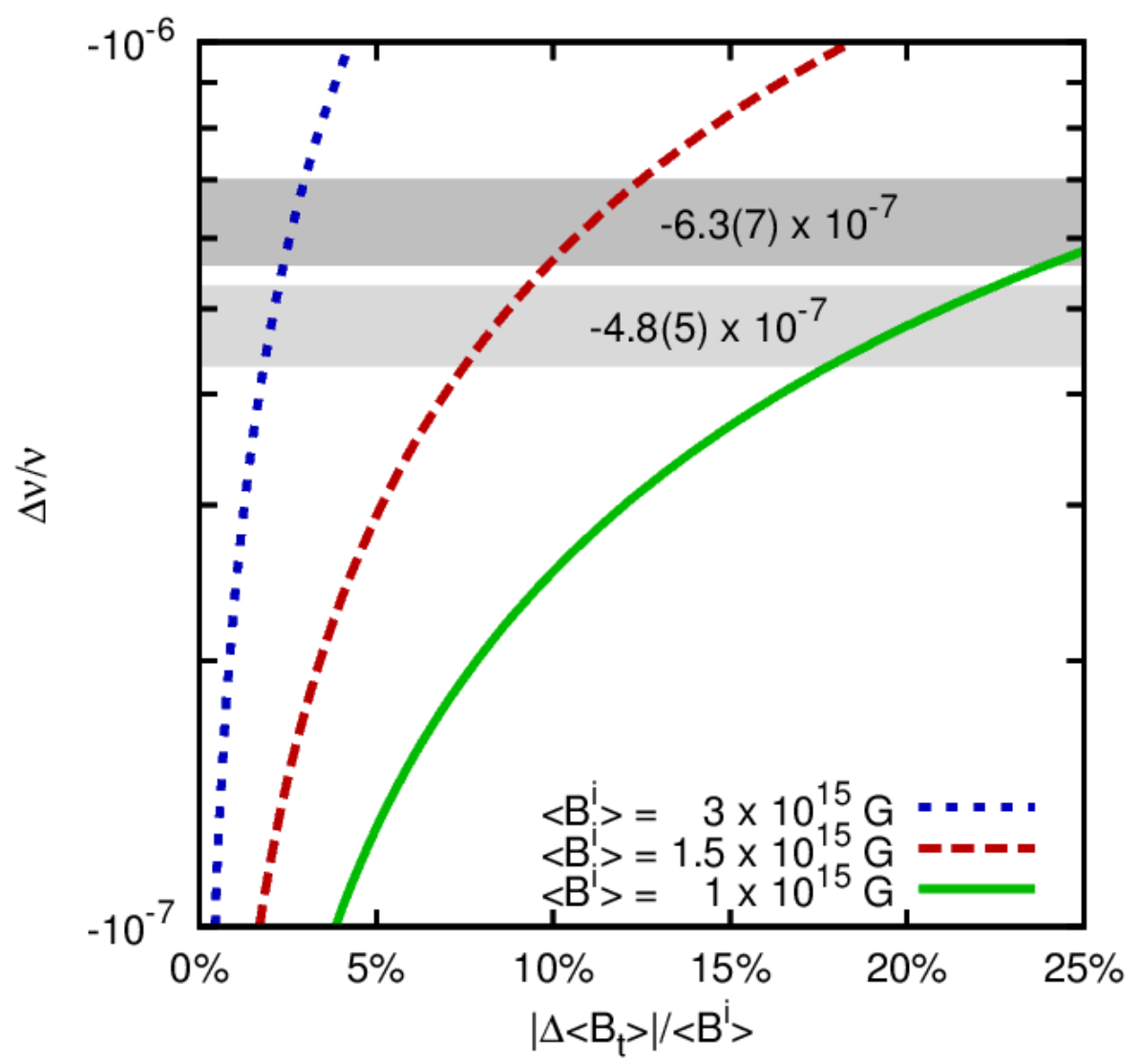

Figura 3.2: Valores de $\Delta v / v$ obtenidos de la Ecuación 3.5 como función del cambio relativo en la intensidad media del campo magnético toroidal $\left|\Delta\left\langle B_{t}\right\rangle\right| /\left\langle B_{t}^{i}\right\rangle$. Como referencia, mostramos además los valores de $\Delta v / v$ del par anti-glitch/anti-glitch observado en AXP 1E 2259+586 usando rectángulos grises que incluyen las barras de error correspondientes.

frecuencia observado en AXP 1E 2259+586. Nos concentramos ahora en el análisis de la energética asociada.

Para una NS "típica", siguiendo lo propuesto por Baym \& Pines (1971) para una corteza estándar, estimamos una liberación de energía gravitacional del orden de $\sim 10^{42} \mathrm{erg}$, mientras que las contribuciones provenientes de la energía rotacional y de esfuerzos acumulados, resultan varios órdenes de magnitud menores. Considerando un SGR típico, la liberación de energía magnética resulta $\sim 10^{41} \mathrm{erg}$. Por lo tanto, la energía total emitida una vez producido el anti-glitch en ondas gravitacionales, partículas y radiación electromagnética, debería resultar del orden de $10^{42} \mathrm{erg}$ que es compatible con las detecciones realizadas con Fermi/GBM y Swift. 


\subsection{Discusión}

Presentamos aquí un modelo simple para explicar el anti-glitch observado en el magnetar AXP 1E 2259+586. Proponemos que un decaimiento natural de la componente toroidal de su campo magnético, de aproximadamente un $\sim 10 \%$, desde un valor inicial del orden de $\left\langle B_{t}^{i}\right\rangle \gtrsim 10^{15} \mathrm{G}$, debería ser suficiente para desestabilizar una configuración originalmente estable y alargada, produciendo la fractura de la estructura estelar reduciéndola a una nueva forma más esférica estable bajo la configuración de campo magnético final. Como resultado, el cambio repentino de los momentos de inercia de la estrella produciría un frenado neto, como el observado en (Archibald et al., 2013), dada la conservación del momento angular. En este escenario, además, considerando una NS típica, estimamos una liberación de energía de $\sim 10^{42} \mathrm{erg}$, en completo acuerdo con la emisión detectada por los observatorios Fermi y Swift en la época correspondiente al evento en cuestión.

Nuestro modelo predice que un anti-glitch como el detectado por Archibald et al. (2013) en AXP 1E 2259+586, sólo puede ocurrir si la intensidad media del campo magnético toroidal interno de la NS es varias veces superior a $10^{14} \mathrm{G}$, como en el caso de los magnetares. Este valor crítico impide el desarrollo de un anti-glitch de esta magnitud en la vasta población de pulsares corrientes propulsados por rotación, intensamente monitoreados durante $\sim 50$ años, lo que podría ser la razón por la cual este evento es el primero en su tipo, ya que la población de magnetares conocidos es varios órdenes de magnitud inferior (10 a 20 miembros) y ha sido monitoreada sólo durante los últimos $\sim 20$ años.

Como una extensión de nuestro modelo simple, el segundo evento reportado por Archibald et al. (2013) podría ser explicado en el contexto de un escenario más complejo, donde el primer frenado repentino podría ser seguido por una serie de réplicas, como ocurre normalmente en los terremotos. Suponiendo $\left\langle B_{t}^{i}\right\rangle=2 \times 10^{15} \mathrm{G}$, por medio de la Ecuación 3.6, estimamos $\left\langle B_{t}^{f}\right\rangle \approx$ $1.87 \times 10^{15} \mathrm{G}(\Delta B \sim 6.5 \%)$ para el primer evento y $\left\langle B_{t}^{f}\right\rangle \approx 1.78 \times 10^{15} \mathrm{G}(\Delta B \sim 11 \%)$ si consideramos ambos eventos como uno compuesto. Como no podemos ir más allá de la resolución temporal de dos observaciones consecutivas, no podemos asegurar cómo estos anti-glitches se comportan en lapsos más breves. De los datos presentados en (Archibald et al., 2013), no puede determinarse si el segundo evento es un segundo frenado o un glitch normal. El estudio detallado de la dinámica en detalle queda por fuera de nuestro modelo.

Resulta importante destacar finalmente que en este escenario, son esperables ondas gravitacionales potencialmente observables por el experimento LIGO avanzado, tanto durante el frenado como por oscilaciones inducidas por los cambios de la estructura estelar. 


\section{Parte III}

\section{Estudios observacionales de estrellas de neutrones}





\section{Breve descripción de la tercera parte}

En esta parte de la tesis presentamos los resultados obtenidos a partir de estudios observacionales llevados a cabo en la banda de los rayos X, utilizando observaciones disponibles en bases de datos de los telescopios RXTE, XMM-Newton y Chandra tendientes a realizar aportes en dos ramas de investigación. El primer caso, involucra la búsqueda de un remanente compacto en los restos de una explosión de supernova, mientras que el segundo trata sobre una NS en acreción dentro de un LMXB.

El Capítulo 4 está dedicado a la búsqueda de un remanente compacto, posiblemente un CCO, en el SNR G290.1-0.8, lo cual incluye el análisis de la emisión extendida de dicha fuente a partir de observaciones de los satélites XMM-Newton y Chandra. Estos resultados fueron publicados en un artículo de la revista Astronomy and Astrophysics (García et al., 2012a), y en el Boletín de la Asociación Argentina de Astronomía (García et al., 2012b).

En el Capítulo 5 mostramos los resultados del estudio del enfriamiento de la NS en el sistema binario $4 \mathrm{U}$ 1820-30, posterior a las erupciones de rayos $\mathrm{X}$ de Tipo-I ocurridas durante los períodos de acreción o actividad de esta fuente. El trabajo incluye la búsqueda y detección de estas erupciones en todas las observaciones de archivo del satélite RXTE. Los resultados de este estudio fueron publicados en un artículo de la revista Monthly Notices of the Royal Astronomical Society (García et al., 2013). 


\section{Capítulo 4}

\section{Búsqueda de un remanente compacto en el interior del SNR G290.1-0.8}

\subsection{Resumen}

El origen y la evolución de los SNRs de morfología mixta dista de ser conocido en profundidad. Varios remanentes presentan morfología de radio distorsionada o capas de emisión de rayos $\mathrm{X}$ con estructuras tipo jet, siendo G290.1-0.8 (MSH 11-61A) uno de ellos. En este capítulo presentamos un estudio detallado de su naturaleza, con el objeto de develar el origen de su morfología particular y realizar una búsqueda de candidatos a remanentes compactos en su interior. Este estudio se llevó a cabo usando información en diversas longitudes de onda del espectro que incluyeron observaciones del plasma caliente en los rayos $\mathrm{X}$ realizadas con los satélites XMM-Newton y Chandra y de las condiciones impuestas por el medio interestelar frío en el que el remanente se expande, utilizando datos de radio y emisión en la línea de H I de $21 \mathrm{~cm}$. Se realizó un análisis espectral y un análisis morfológico con mapas de energía media de los fotones para obtener parámetros físicos y geométricos de la fuente, así como mapas de contornos de radio y cubos de datos de H I para analizar el entorno e introducir un escenario astrofísico acorde.

Las imágenes de rayos X obtenidas muestran que el SNR presenta dos extremos opuestos brillantes sobre un eje de simetría en la dirección noroeste-sudeste. El análisis espectral confirma que las condiciones físicas del plasma son fuertemente inhomogéneas a lo largo del remanente. De hecho, mientras que los dos extremos brillantes tienen temperaturas altas y están fuera del equilibrio de ionización, la parte central del remanente se encuentra más fría y en equilibrio. También, los datos de radio muestran que el remanente se expande dentro de una estructura tubular de baja densidad alineada en la misma dirección. Esta evidencia favorece la idea que la morfología observada es consecuencia directa de la estructura del medio interestelar donde evolucionó el SNR por lo que sugerimos que la explosión debida al colapso de una estrella de gran masa con un fuerte viento dipolar podría explicar todas las características de las emisiones analizadas.

Por otro lado, estudiamos la región central del remanente buscando un posible remanente 
estelar compacto asociado. Nuestro análisis reveló tres fuentes posiblemente puntuales en su interior. Dos de ellas son fuentes de rayos X duros, posiblemente núcleos activos de galaxias de fondo. La tercera, que se encuentra muy cerca del centro geométrico proyectado del SNR, es una fuente brillante de rayos X blandos, inmersa en una estructura de emisión difusa. Del estudio en rayos $\mathrm{X}$ inferimos que la fuente central presenta emisión térmica blanda, sin pulsaciones ni nebulosa asociada al viento de un pulsar. Tampoco encontramos contrapartidas en otras longitudes de onda. Estos resultados sugieren que esta fuente compacta de rayos $\mathrm{X}$ es compatible con las características típicas de un CCO de campo magnético débil, posiblemente asociado al remanente.

\subsection{Introducción}

El origen de los SNRs que presentan una morfología tipo cáscara a frecuencias de radio o rayos $\mathrm{X}$ distorsionada, con dos regiones brillantes simétricas opuestas, es aún poco conocido. Algunos escenarios físicos posibles que permitan explicar la morfología observada incluyen: la expansión de SNRs en múltiples cavidades del medio interestelar (ISM ${ }^{1}$ ) (Braun \& Strom, 1986; Dubner et al., 1994; Milne et al., 1989; Pineault et al., 1987), la expansión de SNRs a través del viento de su estrella progenitora, con distribución de densidad asimétrica (Blondin et al., 1996), o la presencia de una fuente central que produce eyecciones colimadas de material que impactan sobre la cáscara del remanente en dos direcciones opuestas (Murata \& Shibazaki, 1996).

En la Galaxia, hay varios SNRs con características morfológicas de este tipo (Gaensler et al., 1998). SNR G290.1-0.8 es un miembro de esta clase de fuentes peculiares. El objeto fue identificado por primera vez como un SNR sobre la base de su espectro de radio no térmico (Kesteven, 1968). A través de observaciones de radio a $408 \mathrm{MHz}$ (Kesteven \& Caswell, 1987), 5 y $8 \mathrm{GHz}$ (Milne et al., 1989), fue clasificado como un SNR del tipo cáscara, con un tamaño angular de $15 \times 10$ minutos de arco y un índice espectral $\alpha=-0.4\left(S \propto v^{\alpha}\right)$. Usando datos del continuo de radio y de CO Filipovic et al. (2005), estudiaron su morfología y cinemática en detalle. Estos autores encontraron que el SNR está asociado con una nube molecular densa ubicada al suroeste. Más recientemente, Reynoso et al. (2006), usando observaciones de la línea del H I y del continuo de radio a $20 \mathrm{~cm}$, observados con el Australian Telescope Compact Array (ATCA), mostraron que la distribución del gas y la cinemática delante del SNR son complejas y estimaron que probablemente esté localizado en el brazo de Carina, a una distancia de $7 \pm 1 \mathrm{kpc}$.

En los rayos X, G290.1-0.8 fue estudiado por primera vez por Seward (1990) con el observatorio Einstein. Estas observaciones permitieron establecer la naturaleza de centro lleno de la emisión de rayos X, morfológicamente diferente a su emisión en radio. Más tarde, Slane et al. (2002) analizaron observaciones del Advanced Satellite for Cosmology and Astrophysics (ASCA) del objeto determinando que la emisión de rayos $\mathrm{X}$ es de naturaleza térmica, clasificando además al remanente como miembro de la clase de morfología mixta (ver, por ejemplo, Rho \& Petre, 1998), y estimando su edad en el rango de 10 a 20 mil años.

Sin embargo, la pregunta acerca del origen y evolución de G290.1-0.8 y su morfología par-

\footnotetext{
${ }^{1}$ Del inglés, interstellar medium.
} 
Tabla 4.1: Detalles de las observaciones de Chandra y XMM-Newton del SNR G290.1-0.8.

\begin{tabular}{lcclcc}
\hline \hline Satélite & \multicolumn{2}{c}{ Chandra } & & \multicolumn{2}{c}{ XMM-Newton } \\
\cline { 2 - 3 } \cline { 5 - 6 } Obs-Id & 2754 & 3720 & & 0111210201 & 0152570101 \\
Fecha & $16 / 07 / 2002$ & $21 / 07 / 2002$ & & $28 / 07 / 2002$ & $21 / 07 / 2002$ \\
Inicio [UTC] & $03: 12: 39$ & $01: 45: 38$ & & $02: 29: 26$ & $16: 56: 39$ \\
Cámara & ACIS-235678 & ACIS-235678 & & pn/MOS1,2 & MOS1, 2 \\
Filtro & -- & -- & & MEDIUM & MEDIUM \\
Modos (lectura/datos) & TIMED/VFAINT & TIMED/VFAINT & & PFWE & PFWE \\
Exposición [ks] & 29.71 & 33.66 & & $6.04-10.8-10.8$ & $62.2-62.3$ \\
GTI [ks] & 26.04 & 30.59 & & $5.89-10.6-10.6$ & $60.6-60.6$ \\
\hline
\end{tabular}

Todas las observaciones fueron tomadas de las bases de datos disponibles en la red. PFWE se refiere al modo de observación Prime Full Window Extended. Las observaciones fueron centradas en $\alpha=11^{h} 02^{m} 57^{s} .20, \delta=-60^{\circ} 54^{\prime} 34^{\prime \prime}$ (J2000.0).

ticular no tiene una respuesta definitiva. En este trabajo presentamos los resultados del análisis de observaciones de XMM-Newton y Chandra de la fuente, focalizados en el estudio de sus características físicas y químicas, con el objetivo de introducir un escenario astrofísico realista que permita describir el origen de su morfología asimétrica y realizar una búsqueda de una fuente compacta asociada en su interior, no detectada hasta el momento.

\subsection{Observaciones y reducción de datos}

El campo de G290.1-0.8 fue observado por XMM-Newton dos veces. Las observaciones fueron realizadas con la cámara European Photon Imaging Camera (EPIC) que consta de tres detectores: dos cámaras MOS (Turner et al., 2001) y una cámara PN (Strüder et al., 2001) que operan en el rango de energía de 0.2 a $15 \mathrm{keV}$. Ambas observaciones fueron centradas en $\alpha=11^{h} 03^{m} 00^{s} .0$, $\delta=-60^{\circ} 54^{\prime} 00^{\prime \prime} ;$ J2000.0), con el SNR situado en CCD central. En este trabajo analizamos también dos observaciones de Chandra obtenidas con la cámara Advanced CCD Image Spectrometer (ACISI) que opera en el rango de energía de 0.1 a $10.0 \mathrm{keV}$ con alta resolución espacial ( 0.5").

Este gran conjunto de datos brinda la posibilidad, por primera vez, de llevar a cabo un análisis detallado del SNR G290.1-0.8. Los datos de XMM-Newton se analizaron con la versión 11.0.0 del Science Analysis System (SAS), usando las últimas calibraciones disponibles. Las observaciones de Chandra fueron reprocesadas con la versiones 4.1.2 de CIAO y 3.2.2 de CALDB. Para excluir los períodos de altos niveles de fondo, que podrían haber afectado las observaciones, se extrajeron curvas de luz para fotones con energías por encima de los $10 \mathrm{keV}$ de todas las cámaras y se excluyeron intervalos con emisión $3 \sigma$ por encima del promedio para producir archivos $\mathrm{GTI}^{2}$. En la

\footnotetext{
${ }^{2}$ del inglés, Good Time Interval.
} 
Tabla 4.1, mostramos información detallada de las observaciones y características del instrumental usado.

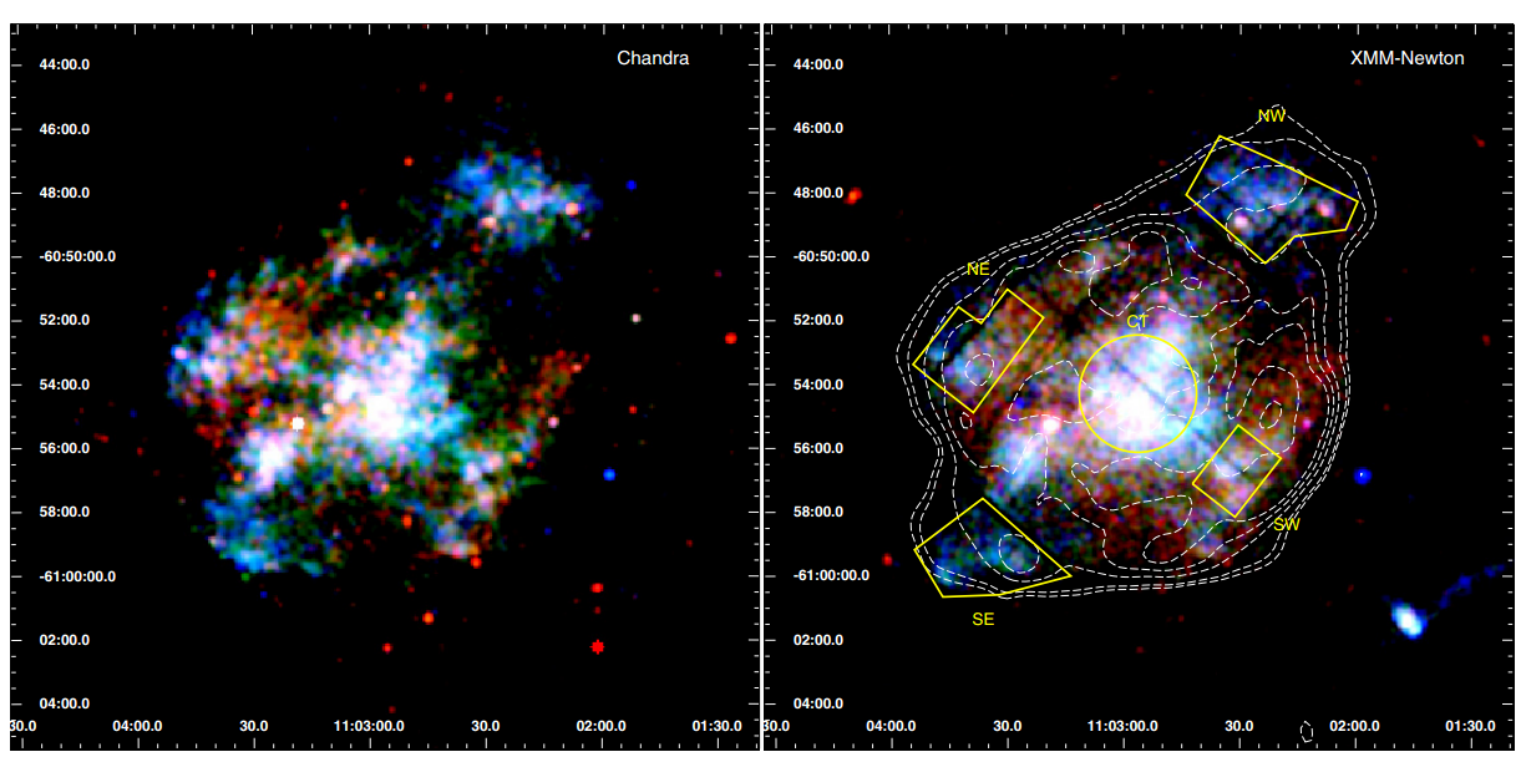

Figura 4.1: Imágenes Chandra (panel izquierdo) y XMM-Newton (panel derecho) del SNR G290.1-0.8 en tres bandas de energía de rayos X: blandos $(0.5-1.2 \mathrm{keV})$ en rojo, medio (1.2$1.8 \mathrm{keV})$ en verde, y duros (1.8-2.8 keV) en azul. El panel derecho muestra también los contornos de radio a $843 \mathrm{MHz}$ (Whiteoak \& Green, 1996) superpuestos en blanco. Las 5 regiones seleccionadas para la extracción de fotones en el estudio espectral son también indicadas en amarillo. En ambas imágenes el Norte está hacia arriba y el Este hacia la izquierda.

\subsection{Resultados}

\subsubsection{Imágenes de rayos $\mathrm{X}$}

La resolución espacial y sensibilidad disponible en las observaciones de rayos X analizadas nos permiten examinar en profundidad la morfología en rayos X de G290.1-0.8. En la Figura 4.1 presentamos imágenes producidas a partir de los datos de Chandra (panel izquierdo) y $X M M$ Newton (panel derecho) en tres bandas de energía de rayos X: blandos $(0.5-1.2 \mathrm{keV})$ en rojo, medios $(1.2-1.8 \mathrm{keV})$ en verde, y duros $(1.8-2.8 \mathrm{keV})$ en azul. En el panel derecho de la Figura 4.1 se incluyen también los contornos de radio a $843 \mathrm{MHz}$ superpuestos en color blanco. Estos contornos de radio corresponden a datos extraídos del catálogo de SNRs (Whiteoak \& Green, 1996) realizado con observaciones del Molonglo Observatory Synthesis Telescope (MOST). Para generar las imágenes de rayos X de XMM-Newton, utilizamos los datos de ambas cámaras MOS, corregidos por exposición y degradación (conocida como viñeteo), y aplicamos un filtro gaussiano con un radio de 3 píxeles.

Las imágenes XMM-Newton y Chandra revelan detalles de la estructura en rayos X de G290.1- 
Tabla 4.2: Parámetros espectrales de la emisión de rayos X difusa de las regiones seleccionadas.

\begin{tabular}{l|ccccc}
\hline \hline Modelo \& Parámetros & Región CT & Región SW & Región NE & Región NW & Región SE \\
\hline PHABS*VPSHOCK & & & & & \\
$N_{\mathrm{H}}\left[10^{22} \mathrm{~cm}^{-2}\right]$ & $0.62 \pm 0.01$ & $0.61 \pm 0.03$ & $0.50 \pm 0.02$ & $0.43 \pm 0.02$ & $0.61 \pm 0.04$ \\
$\mathrm{kT}[\mathrm{keV}]$ & $0.593 \pm 0.008$ & $0.57 \pm 0.02$ & $0.63 \pm 0.02$ & $0.89 \pm 0.03$ & $0.84 \pm 0.05$ \\
$\mathrm{O}\left[\mathrm{O}_{\odot}\right]$ & $0.61 \pm 0.06$ & $0.9 \pm 0.2$ & $0.4 \pm 0.1$ & $0.13 \pm 0.07$ & $0.3 \pm 0.1$ \\
$\mathrm{Ne}\left[\mathrm{Ne}_{\odot}\right]$ & $0.26 \pm 0.03$ & $0.33 \pm 0.08$ & $0.17 \pm 0.06$ & $0.13 \pm 0.08$ & $0.07 \pm 0.05$ \\
$\mathrm{Mg}\left[\mathrm{Mg}_{\odot}\right]$ & $0.95 \pm 0.03$ & $0.81 \pm 0.07$ & $0.68 \pm 0.05$ & $0.60 \pm 0.06$ & $0.61 \pm 0.06$ \\
$\mathrm{Si}\left[\mathrm{Si}_{\odot}\right]$ & $1.76 \pm 0.04$ & $1.5 \pm 0.1$ & $1.18 \pm 0.07$ & $1.15 \pm 0.06$ & $1.35 \pm 0.09$ \\
$\mathrm{~S}\left[\mathrm{~S}_{\odot}\right]$ & $1.4 \pm 0.1$ & $1.3 \pm 0.3$ & $1.13 \pm 0.2$ & $1.0 \pm 0.1$ & $1.0 \pm 0.2$ \\
$\mathrm{Fe}\left[\mathrm{Fe}_{\odot}\right]$ & $0.080 \pm 0.005$ & $0.12 \pm 0.01$ & $0.09 \pm 0.01$ & $0.02 \pm 0.01$ & $0.02 \pm 0.01$ \\
$\tau\left[10^{13} \mathrm{~s} \mathrm{~cm}{ }^{-3}\right]$ & $>5$ & $>5$ & $4.15 \pm 0.5$ & $0.12 \pm 0.08$ & $0.03 \pm 0.01$ \\
Norm $\left[10^{-3}\right]$ & $13.2 \pm 0.4$ & $2.3 \pm 0.2$ & $4.4 \pm 0.2$ & $3.0 \pm 0.2$ & $2.0 \pm 0.3$ \\
\hline$\chi_{v}^{2} / \mathrm{d}$. o.f. & $1.58 / 345$ & $1.13 / 337$ & $1.00 / 343$ & $1.32 / 342$ & $1.12 / 340$ \\
\hline Flujo $(0.3-1.0 \mathrm{keV})$ & $84.5 \pm 0.5$ & $18.0 \pm 0.4$ & $25.5 \pm 0.4$ & $17.2 \pm 0.3$ & $24.1 \pm 0.5$ \\
Flujo $(1.0-2.0 \mathrm{keV})$ & $35.9 \pm 0.2$ & $6.18 \pm 0.2$ & $10.3 \pm 0.2$ & $9.07 \pm 0.2$ & $6.70 \pm 0.2$ \\
Flujo $(2.0-3.0 \mathrm{keV})$ & $5.28 \pm 0.04$ & $0.80 \pm 0.02$ & $1.71 \pm 0.03$ & $2.59 \pm 0.05$ & $1.42 \pm 0.03$ \\
\hline Flujo total $(0.3-3.0 \mathrm{keV})$ & $126 \pm 1$ & $25.0 \pm 0.6$ & $37.5 \pm 0.6$ & $28.8 \pm 0.5$ & $32.2 \pm 0.7$ \\
\hline
\end{tabular}

La normalización se define como $10^{-14} / 4 \pi \mathrm{D}^{2} \times \int n_{H} n_{e} d V$, donde $D$ es la distancia en $[\mathrm{cm}], \mathrm{n}_{\mathrm{H}}$ es densidad de hidrógeno $\left[\mathrm{cm}^{-3}\right], n_{e}$ es densidad electrónica $\left[\mathrm{cm}^{-3}\right]$, y $V$ es el volumen $\left[\mathrm{cm}^{3}\right]$. Los flujos están dados en $10^{-13} \mathrm{erg} \mathrm{cm}^{-2} \mathrm{~s}^{-1}$ y están corregidos por absorción y los valores del error están dados al $90 \%$ para cada parámetro. Las abundancias son relativas a los valores solares dados por Anders \& Grevesse (1989).

0.8 que no habían sido detectados en estudios de rayos X previos (Slane et al., 2002). La morfología global del SNR es alargada en el eje noroeste/sureste con dos regiones brillantes opuestas separadas de la componente difusa central. En la dirección noreste/suroeste se observan dos estructuras con forma de "orejas", diametralmente opuestas con respecto al centro geométrico del remanente. La emisión de rayos X extendida llena todo el interior del borde de la cáscara observada en radio y es prominente a energías de rayos $\mathrm{X}$ medias y duras. Las partes sur, norte y las componentes con forma de "orejas" son más brillantes a energías medias y duras en relación a la emisión de rayos X total. En ninguna de las observaciones se detecta emisión del SNR por encima de los $2.8 \mathrm{keV}$.

\subsubsection{Análisis espectral en rayos $\mathrm{X}$}

Con el fin de estudiar detalladamente las condiciones físicas y químicas del plasma, realizamos un análisis espectral de diversas regiones del SNR usando datos de XMM-Newton. En base a la 

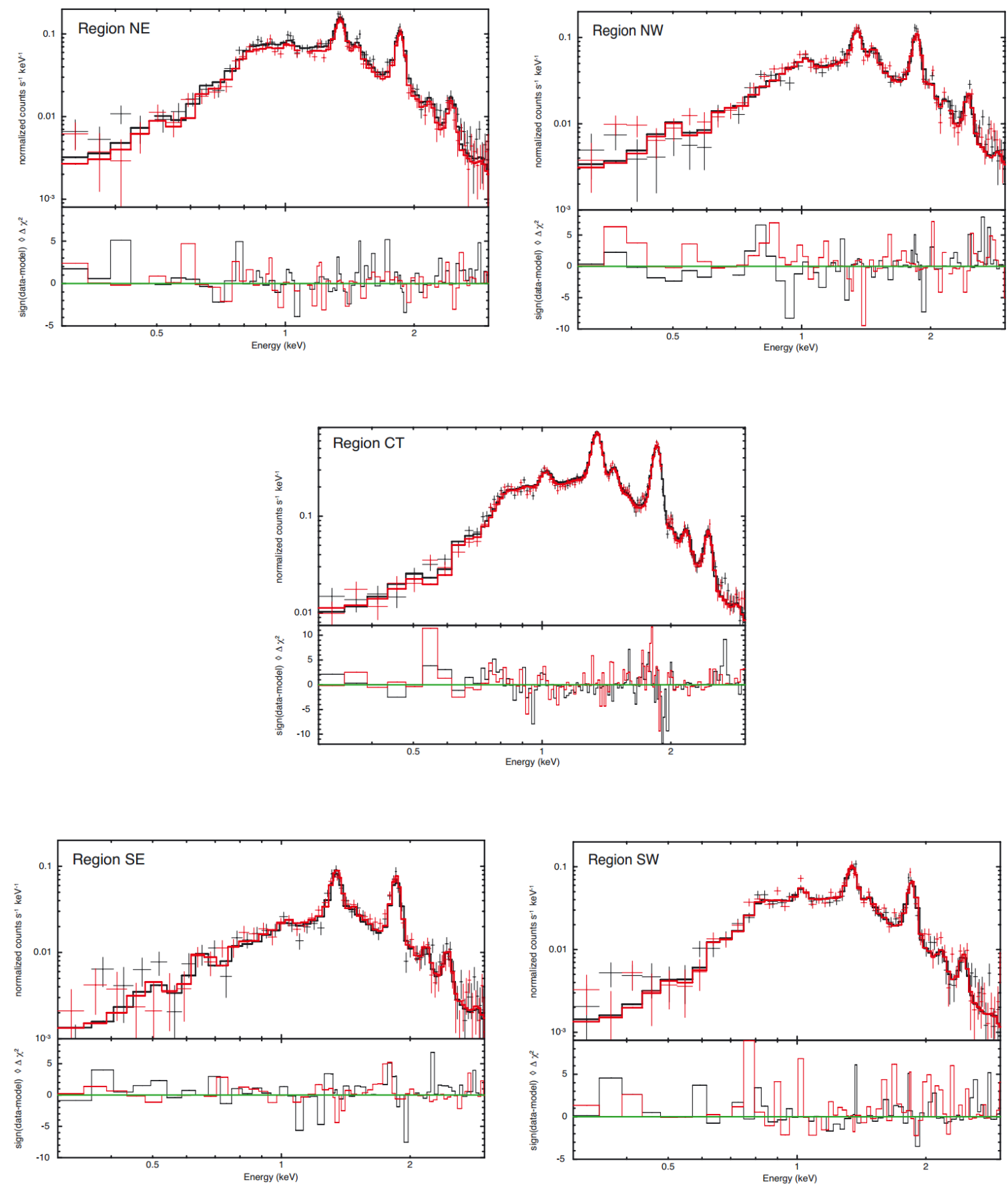

Figura 4.2: Espectros extraídos con los detectores MOS1/2 de XMM-Newton de las 5 regiones seleccionadas (Figura 4.1). Las líneas sólidas indican el modelo VPSHOCK del mejor ajuste (ver Tabla 4.2). El panel inferior muestra los residuos $\chi^{2}$ del ajuste. 

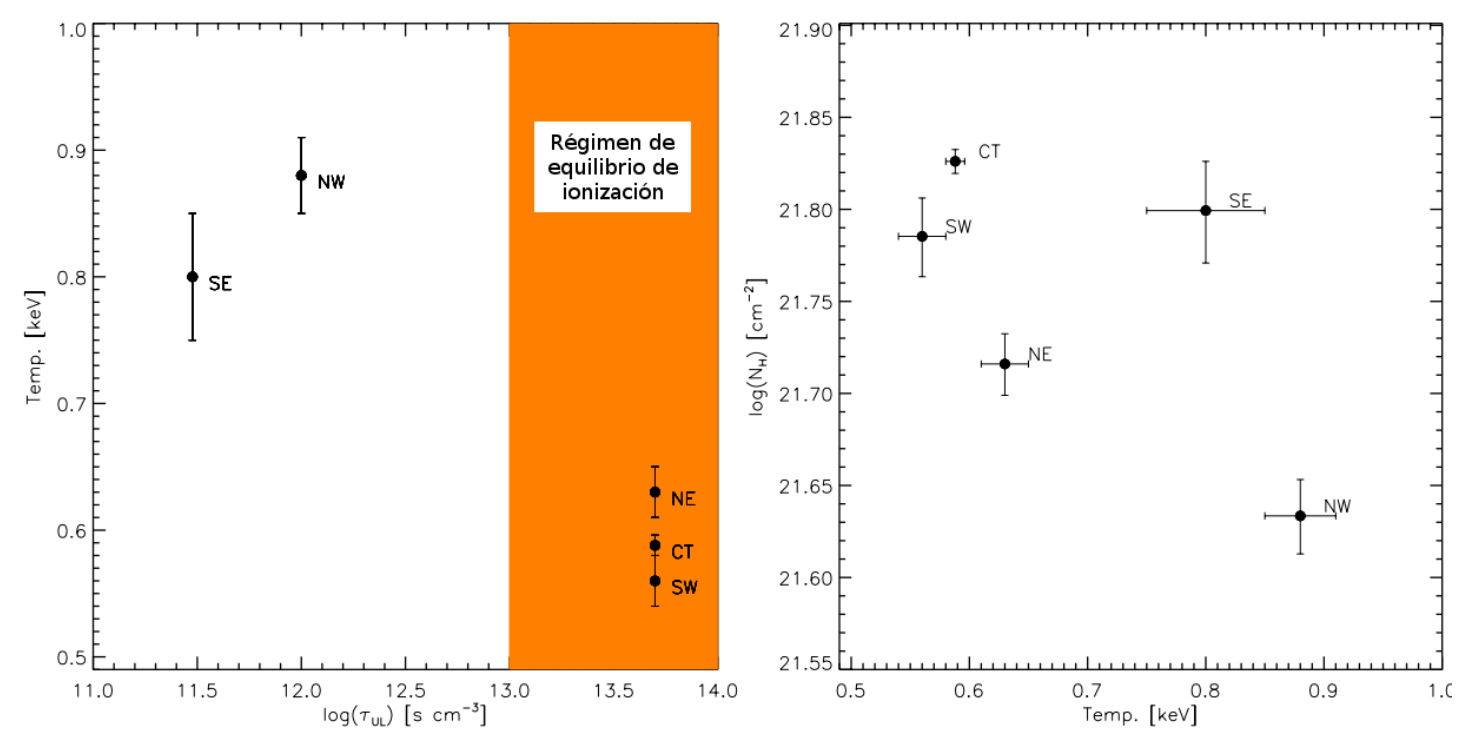

Figura 4.3: Panel izquierdo: Temperatura y escala temporal de ionización $\left(\tau_{\mathrm{ul}}\right)$ de las 5 regiones seleccionadas. El régimen de equilibrio $\left(\tau_{\mathrm{ul}}>10^{13} \mathrm{~s} \mathrm{~cm}^{-3}\right)$ se resalta en color anaranjado. Panel derecho: distribución de la temperatura y absorción $\left(N_{\mathrm{H}}\right)$ obtenidos para las regiones consideradas en el estudio.

morfología observada a partir de las imágenes de rayos $\mathrm{X}$, se extrajeron espectros a lo largo de la dirección del eje noroeste/sureste de tres regiones indicadas como: Sudeste (SE), Central (CT) y Noroeste (NW), y para otras dos zonas ubicadas perpendicularmente, nombradas: Sudoeste (SW) y Noreste (NE), indicadas en el panel derecho de la Figura 4.1. Estas regiones fueron elegidas con un tamaño suficientemente grande como para tener una buena estadística de fotones y lo suficientemente pequeña como para poder discernir las diferentes condiciones del plasma. Los espectros fueron extraídos mediante la tarea EVSELECT de SAS con los parámetros adecuados para las cámaras MOS $1 / 2$. Las matrices de área efectiva (ARF) y respuesta (RMF) fueron obtenidas a través de la tareas ARFGEN y RMFGEN de SAS, respectivamente. El fondo fue sustraído usando los archivos blank sky de XMM-Newton (Carter \& Read, 2007) para las mismas cinco regiones.

La Figura 4.2 muestra los espectros corregidos por absorción de cada región obtenidos a partir de los datos de la cámara MOS1/2 de XMM-Newton. En los gráficos, los espectros fueron agrupados a un mínimo de 16 cuentas por bin. Las barras de error corresponden al nivel del $90 \%$ para la estadística de $\chi^{2}$. El análisis espectral se realizó mediante el paquete de software XSPEC (Arnaud, 1996), trabajando en el rango de energía 0.3-3.0 keV, ya que el SNR no emite por encima de este valor.

Los espectros de rayos X están dominados por líneas de emisión sobre un continuo. Usando la guía interactiva $\mathrm{ATOMDB}^{3}$, identificamos las líneas más importantes del espectro correspondientes a transiciones atómicas de O VII (0.66 keV), Ne IX (0.91 keV), Mg XII (1.47 keV), Si XIII

\footnotetext{
${ }^{3}$ http://cxc.harvard.edu/atomdb/WebGUIDE/index.html
} 
(1.85 keV), S CXV (2.4 keV) y Fe XXI (0.99 keV). Sin embargo, debido a la resolución espectral moderada de las cámaras MOS, la mayoría de las líneas se ven fuertemente afectadas por mezcla, sesgando así la determinación de la identificación y abundancia de los elementos químicos individuales.

Los espectros de la emisión difusa fueron ajustados usando distintos modelos: desde un modelo simple de bremsstrahlung con líneas gaussianas, hasta modelos de plasmas térmicos complejos como VMEKAL, VNEI o VPSHOCK, cada uno modificado por una componente de absorción interestelar (PHABS; Balucinska-Church \& McCammon, 1992) y leyes de potencia para dar cuenta de emisión no térmica asociada al SNR. Después de varias pruebas, se determinó que el mejor ajuste se obtiene utilizando un modelo VPSHOCK, lo que confirma la naturaleza térmica del plasma emisor, como destacaran Slane et al. (2002).

Los parámetros del mejor ajuste de los espectros de emisión difusa en las cinco regiones se enumeran en la Tabla 4.2. En la Figura 4.3 se resaltan varios resultados importantes que se deducen del análisis espectral. Por un lado, las regiones nombradas como CT, NE y SW muestran valores altos de la escala temporal de ionización $\left(\tau \sim 5 \times 10^{13} \mathrm{~s} \mathrm{~cm}^{-3}\right)$ sugiriendo que el plasma ha alcanzado el equilibrio de ionización, mientras que las zonas NW y SE muestran valores relativamente bajos $\left(\tau \lesssim 10^{12} \mathrm{~s} \mathrm{~cm}^{-3}\right)$, lo que indica que el plasma caliente aún no ha alcanzado el equilibrio en estas regiones (ver panel izquierdo de la Figura 4.3). Los valores de $N_{\mathrm{H}}$ obtenidos para cada región son similares. Sin embargo, es interesante observar que el valor de $N_{\mathrm{H}}$ en la región NW es un 30\% menor que en la región opuesta SE. Esto indica que el material a lo largo de la línea de visual varía dependiendo del ángulo, en concordancia con un medio denso en el cual el SNR se expande. Por otra parte, las temperaturas obtenidas para las regiones CT, SW y NE son significativamente inferiores a las temperaturas obtenidas para las zonas NW y SE (ver panel derecho de la Figura 4.3).

En la Tabla 4.2, se pueden analizar también los valores de las abundancias químicas obtenidas para las regiones CT, NE/SW y NW/SE. Las diferencias en las columnas de absorción y abundancia de metales que se muestran aquí con respecto al trabajo previo de Slane et al. (2002) pueden explicarse en los siguientes términos. En primer lugar, porque en este trabajo utilizamos datos de XMM-Newton que tienen suficiente estadística como para producir espectros con alta resolución espacial. En segundo lugar, debido al modelo espectral utilizado. En su artículo, Slane et al. (2002) añadió una ley de potencia a la componente térmica con el fin de ajustar los datos de rayos X duros. Esta ley de potencia fenomenológica se extiende hasta los rayos X blandos, produciendo un exceso suave el cual debería ser disminuido por la alta columna de absorción para ajustar los datos de la parte blanda. En nuestro estudio, sin embargo, los datos XMM-Newton pudieron ajustarse bien usando solamente un modelo térmico, sin ley de potencia, dando sistemáticamente columnas de absorción más bajas. Además, ya que el peso de la componente térmica se incrementa en nuestro ajuste, debido a la ausencia de la ley de potencia, los perfiles de línea de emisión se ajustan con valores menores de las abundancias.

De los flujos de rayos X corregidos por absorción obtenidos para cada una de las regiones en los tres rangos de energía deducimos que el remanente es más intenso ( $70 \%$ del total) a energías bajas, mientras que el resto de la emisión se reparte entre los rayos $\mathrm{X}$ medios y duros. En las 
regiones del NW y SE el cociente de los rayos X duros sobre los medios resulta $~ 0.25$, mientras que en las zonas de NE y SW esta relación se convierte en $\sim 0.15$. Por lo tanto, la emisión de los dos extremos brillantes opuestos resulta más dura que en el resto del SNR, en acuerdo con el análisis de las imágenes.

La medida de emisión (EM) de las cinco regiones seleccionadas permite estimar la densidad y masa correspondiente al plasma emisor de rayos X. Basado en la imagen de rayos X, podemos groseramente asumir que la emisión de rayos X llena las regiones mencionadas que consideramos como volúmenes de emisión localizados a una distancia de $7 \mathrm{kpc}$ que resultan: $V_{\mathrm{CT}} \sim 5.5 \times 10^{57} \mathrm{~cm}^{3}$, $V_{\mathrm{NW}} \sim 2.4 \times 10^{57} \mathrm{~cm}^{3}, V_{\mathrm{SE}} \sim 1.8 \times 10^{57} \mathrm{~cm}^{3}, V_{\mathrm{SW}} \sim 0.9 \times 10^{57} \mathrm{~cm}^{3}$ y $V_{\mathrm{NE}} \sim 2.5 \times 10^{57} \mathrm{~cm}^{3}$. Basado en la EM determinada por las normalizaciones obtenidas del ajuste espectral, la densidad electrónica del plasma es aproximadamente $n_{\mathrm{e}}=\sqrt{E M / V}$, dando como resultado $n_{\mathrm{e}}(\mathrm{CT}) \sim n_{\mathrm{e}}(\mathrm{SW}) \sim 1.1 \mathrm{~cm}^{-3}$, $n_{\mathrm{e}}(\mathrm{NW}) \sim n_{\mathrm{e}}(\mathrm{SE}) \sim 1.0 \mathrm{~cm}^{-3}$ y $n_{\mathrm{e}}(\mathrm{NE}) \sim 0.7 \mathrm{~cm}^{-3}$, si se supone que la densidad de nucleones es igual a la densidad de electrones. Por consiguiente, la masa del plasma se puede estimar como $M=n_{\mathrm{e}} m_{\mathrm{H}} V$, donde $m_{\mathrm{H}}$ es la masa del átomo de hidrógeno, de lo cual resulta: $M_{\mathrm{CT}} \sim 5.3 \mathrm{M}_{\odot}$, $M_{\mathrm{NW}} \sim 2.0 \mathrm{M}_{\odot}, M_{\mathrm{SE}} \sim 1.4 \mathrm{M}_{\odot}, M_{\mathrm{SW}} \sim 0.8 \mathrm{M}_{\odot} \mathrm{y} M_{\mathrm{NE}} \sim 1.5 \mathrm{M}_{\odot}$. Estas masas del plasma ionizado, no deben confundirse con la masa atómica o molecular del gas.

Aunque las condiciones físicas en las regiones NW y SE de G290.1-0.8 son diferentes, es posible obtener una estimación de la edad del gas emisor de rayos $\mathrm{X}$ en estas regiones. Esta edad es determinada por la escala temporal de ionización $\tau$, usando $t=\tau / n_{\mathrm{e}}$. A partir de esta relación, el tiempo de enfriamiento del plasma resulta en el rango de entre 10 y 30 mil años. Este resultado concuerda muy bien con la edad del SNR deducida por Slane et al. (2002).

\subsubsection{Mapa de energía media de fotones}

A partir del análisis espectral probamos que las regiones seleccionadas del SNR presentan condiciones de plasma considerablemente diferentes. Lamentablemente, otras áreas del SNR presentan una estadística de fotones limitada que no permite arribar a conclusiones sobre la energética de toda la región de radiación a partir del análisis espectral.

Con el fin de ampliar el estudio de la anisotropía física de la emisión, y obtener un conocimiento cabal de la estructura térmica de todo el SNR, produjimos un mapa de la energía media de fotones (MPE) de todo el SNR. El mapa MPE es una imagen donde el valor de cada píxel corresponde a la energía media de los fotones detectados por los CCDs MOS dentro del rango de energía 0.3-3.0 keV. Este mapa provee información sobre la distribución espacial de las propiedades térmicas del plasma. Para obtener este mapa se fusionaron los archivos de eventos de MOS1 y MOS2, creando una imagen de 9" de resolución que comprende un mínimo de 4 cuentas por píxel. Para cada píxel, se calculó la energía media de los fotones y luego se suavizó el mapa con una gaussiana de 3 píxeles (ver Miceli et al., 2005).

El MPE abarco un rango de 1.1 a $1.9 \mathrm{keV}$ para píxeles con menos de 10 fotones, pero converge a un valor medio total de $1.43 \mathrm{keV}$ para toda la emisión del SNR. Acorde al mapa suavizado de G290.1-0.8, que se muestra en la Figura 4.4, se puede confirmar la presencia fotones con energía media alta $(\sim 1.65 \mathrm{keV})$ en las dos regiones brillantes y opuestas del remanente, mientras que la 


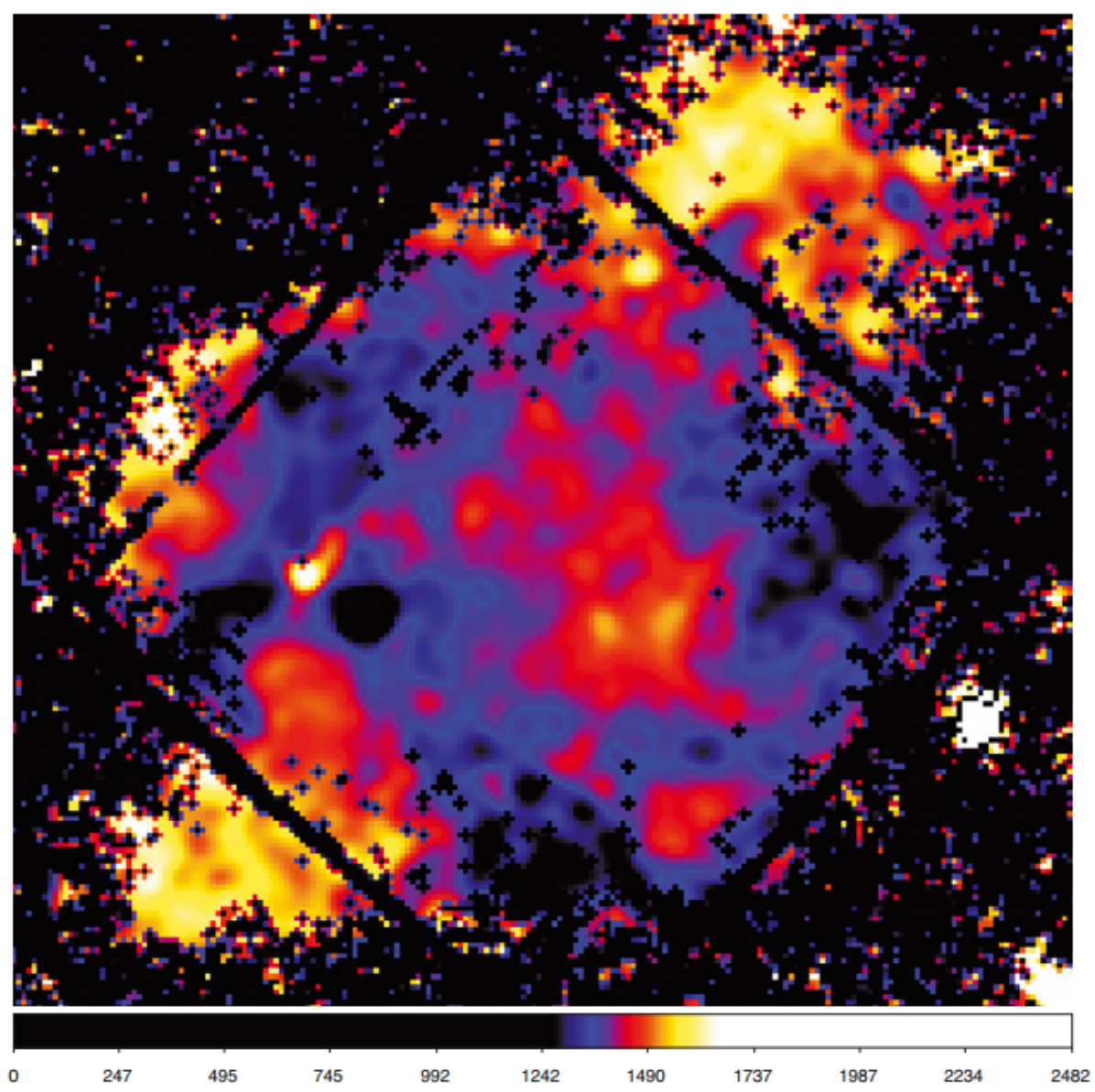

Figura 4.4: Mapa de energía media de fotones MOS entre $0.3-3.0 \mathrm{keV}$. Los píxeles con menos de 4 cuentas han sido descartados. La barra de colores tiene escala lineal y el código de colores representa el rango de energía de $1.2 \mathrm{keV}$ a $1.7 \mathrm{keV}$.

parte central presenta energías medias más bajas $(\sim 1.3 \mathrm{keV})$. Este resultado concuerda muy bien con el análisis espectral obtenido en las secciones previas donde se mostró que las zonas NW y $\mathrm{SE}$ presentan las más altas temperaturas del plasma y flujos de rayos $\mathrm{X}$ más duros que en las regiones $\mathrm{NE}$, SW y CT.

\subsubsection{Análisis del medio interestelar frío a frecuencias de radio}

La observaciones de la línea del H I estudiadas por Reynoso et al. (2006) revelaron que la distribución del gas entorno al SNR G290.1-0.8 tiene una estructura compleja. El SNR se encuentra en una zona de alta densidad del ISM, posiblemente dentro de una gran nube atómica y molecular. Con el propósito de saber si el ISM que circunda al remanente podría estar afectando su evolución, utilizamos datos de la línea del H I pertenecientes al Southern Galactic Plane Survey (SGPS; McClure-Griffiths et al., 2005), para analizar en detalle la distribución de la densidad de 


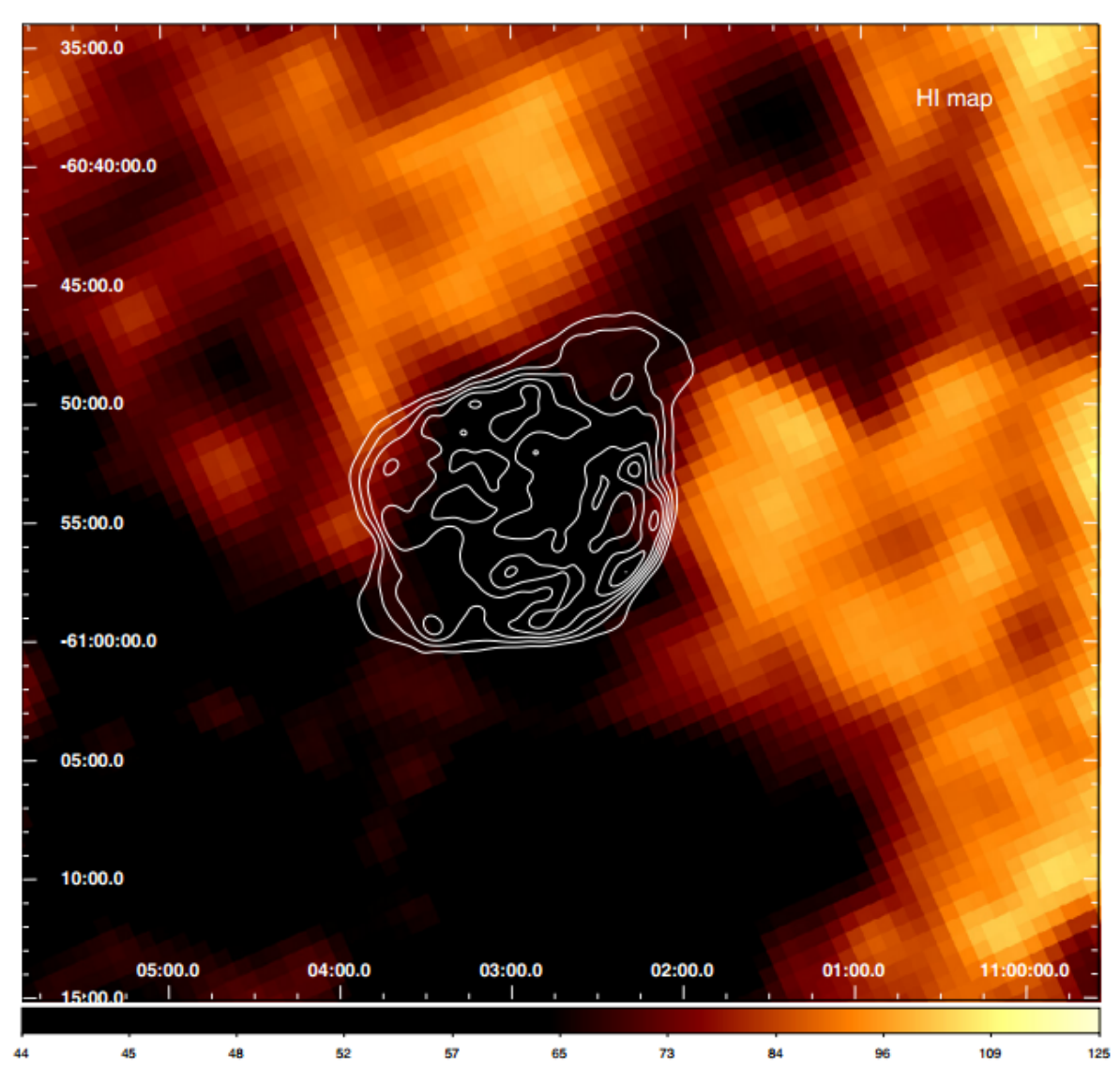

Figura 4.5: Mapa del H I de datos combinados de ATCA y SGPS en la dirección del SNR G290.10.8 para el rango de velocidades de $+5.8 \mathrm{a}+11.5 \mathrm{~km} \mathrm{~s}^{-1}$, con los contornos de radio a $843 \mathrm{MHz}$ de MOST superpuestos. La escala del mapa del H I está en unidades de Kelvin.

gas frío en el medio. La Figura 4.5 muestra un mapa del H I producido para el rango de velocidades de $+5.8 \mathrm{a}+11.5 \mathrm{~km} \mathrm{~s}^{-1}$ con los contornos de radio a $843 \mathrm{MHz}$ de MOST, superpuestos. Este rango de velocidades corresponde a la distancia de $7 \mathrm{kpc}$ donde se encuentra el SNR. En este mapa, tres características importantes pueden observarse: primero, que el contorno de radio exterior coincide muy bien con el borde de la emisión de H I que circunda la cavidad central; segundo, que dos estructuras tubulares alargadas en el borde noroeste se extienden más allá de los límites del borde exterior en radio; tercero, que en la parte suroeste una región de alta densidad parece estar interaccionando con el borde del SNR como sugirieron previamente Filipovic et al. (2005). Si se trata de una estructura preexistente del ISM, esta podría ser responsable de la morfología radio/rayos $\mathrm{X}$ observada.

La emisión tenue de la región central puede explicarse como el resultado del defecto real del H I debido al pasaje del frente de choque de una explosión de supernova o como la absorción de H I producida por la emisión de continuo de radio asociada al SNR. Debido a la ausencia de un modelo detallado de la opacidad del ISM en la región, no hay cálculos fiables como para encontrar 
la distribución de densidad H I en el interior del SNR. Sin embargo, el ISM altamente estructurado donde el SNR está inmerso, en correlación con la morfología de rayos X observada, favorece el escenario de un medio inhomogéneo pre-existente que da lugar a la morfología observada.

\subsubsection{Búsqueda de fuentes puntuales en el interior del SNR G290.1-0.8}

En el panel izquierdo de la Figura 4.6, mostramos una imagen similar a la Figura 4.1, en los tres rangos de energía: blandos $(0.5-1.2 \mathrm{keV})$, medios $(1.2-1.8 \mathrm{keV})$ y duros $(1.8-2.8 \mathrm{keV})$, mientras que en el panel derecho presentamos una ampliación de la región central del SNR. Como puede verse, existen tres fuentes puntuales: 2XMMi J110311.1-605450, 2XMMi J110304.8-605336 y 2XMMi J110255.8-605449 (elipse). Las primeras dos son fuentes intrínsecamente duras, posiblemente asociadas a núcleos activos de galaxias, mientras que la tercera, localizada cerca del pico de intensidad en $\alpha=11^{h} 02^{m} 56^{s} .89, \delta=-60^{\circ} 54^{\prime} 39^{\prime \prime} .64$, es más blanda y no presenta contrapartidas en el óptico ni en el IR. Usando la tarea WAVDETECT de CIAO detectamos la fuente en datos ACIS a un nivel de $5 \sigma$, usando núcleos de $2,4,6$ y 8 píxeles y un límite inferior de $10^{-6}$.

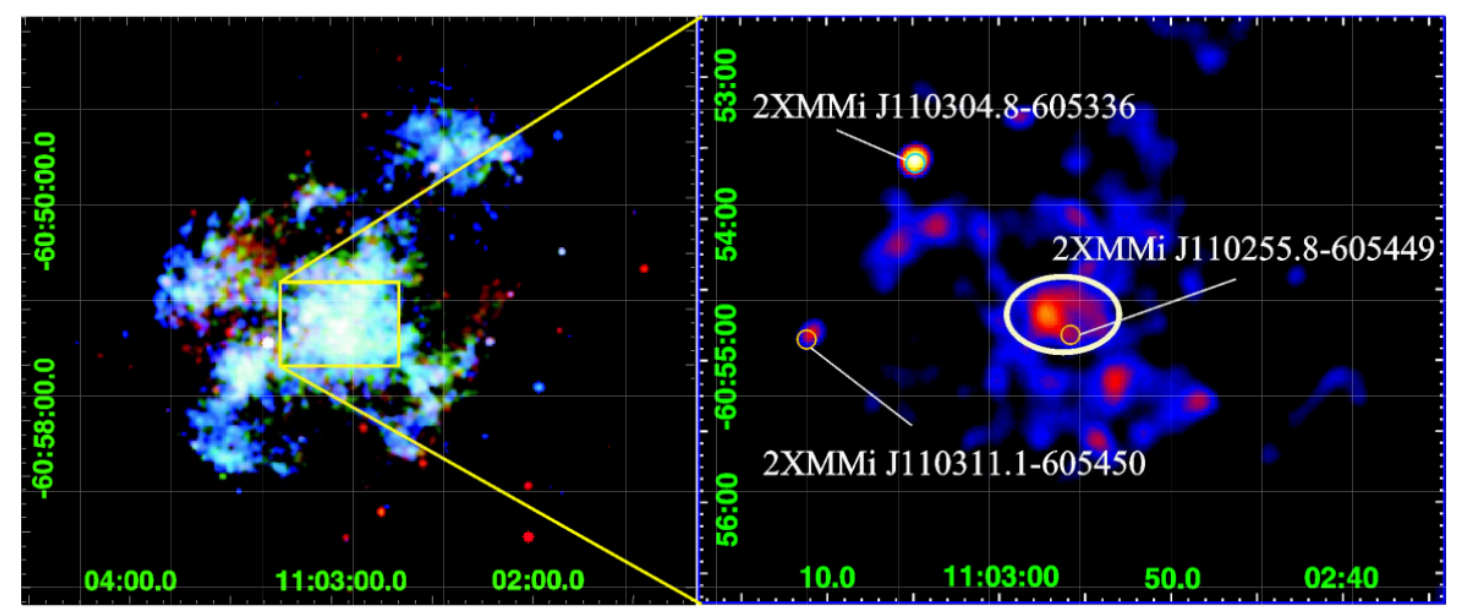

Figura 4.6: Panel izquierdo: Imagen Chandra de SNR G290.1-0.8. Panel derecho: Ampliación de la región central del SNR con las tres fuentes de rayos X compactas indicadas.

Respecto de esta fuente de rayos X puntual localizada en el centro geométrico aparente del SNR, encontramos que no presenta variabilidad significativa, desfavoreciendo un origen asociado a un sistema binario, su espectro térmico es blando, eliminando la posibilidad de un núcleo activo de galaxia, así como ausencia de contrapartida óptica/IR y ausencia de una nebulosa de viento circundante. Estas propiedades son similares a las encontradas en los CCOs o anti-magnetares (ver la Tabla 4.3, basada en Halpern \& Gotthelf, 2010). Con toda esta información en mente, se sugiere que esta fuente de rayos X compacta podría ser un CCO, débilmente magnetizado, situado cerca del centro de G290.1-0.8, y posiblemente asociado físicamente al SNR. 
Tabla 4.3: Propiedades de CCOs conocidos y candidatos en SNRs.

\begin{tabular}{llcccccc}
\hline \hline $\begin{array}{l}\text { CCOs } \\
\text { Confirmados }\end{array}$ & SNR & $\begin{array}{c}\text { Edad } \\
{[\mathrm{kyr}]}\end{array}$ & $\begin{array}{c}d \\
{[\mathrm{kpc}]}\end{array}$ & $\begin{array}{c}k T \\
{[\mathrm{keV}]}\end{array}$ & $\begin{array}{c}R \\
{[\mathrm{~km}]}\end{array}$ & $\begin{array}{c}L_{\mathrm{X}, \mathrm{bol}} \\
{\left[\mathrm{erg} \mathrm{s}^{-1}\right]}\end{array}$ & Ref. \\
\hline RX J0822.0-4300 & Puppis A & 3.7 & 2.2 & $0.21(5)$ & 8.1 & $6.5 \times 10^{33}$ & {$[1]$} \\
CXOU J085201.4-461753 & G266.1-1.2 & 1 & 1 & $0.404(5)$ & 0.3 & $2.5 \times 10^{32}$ & {$[1]$} \\
1E 1207.4-3949 & PKS1209-51 & 7 & 2.2 & $0.163(3)$ & 4.6 & $2.5 \times 10^{33}$ & {$[1]$} \\
CXOU J160103.1-513353 & G330.2+1.0 & $\gtrsim 3$ & 5 & $0.48(3)$ & 0.4 & $1.5 \times 10^{33}$ & {$[1]$} \\
1WGA J1713.4-3949 & G347.3-0.5 & 1.6 & 1.3 & $0.40(1)$ & 2.5 & $\sim 1 \times 10^{33}$ & {$[1]$} \\
CXOU J185238.6+004020 & Kes 79 & 7 & 7 & $0.460(7)$ & 0.7 & $5.3 \times 10^{33}$ & {$[1]$} \\
CXOU J232327.9+584842 & Cas A & 0.33 & 3.4 & $0.40(1)$ & 0.9 & $4.7 \times 10^{33}$ & {$[1]$} \\
\hline Candidatos & & & & & & & \\
\hline XMMU J172054.5-372652 & G350.1-0.3 & 0.9 & 4.5 & $0.50(1)$ & 1.6 & $3.4 \times 10^{33}$ & {$[1]$} \\
XMMU J173203.3-344518 & G353.6-0.7 & $\sim 27$ & 3.2 & 0.5 & 2.0 & $1.0 \times 10^{34}$ & {$[1]$} \\
CXOU J181852.0-150213 & G15.9+0.2 & $1-3$ & 8.5 & $0.4(1)$ & $\ldots$ & $\sim 1 \times 10^{33}$ & {$[1]$} \\
CXOU J170357.8-414302 & G344.7-0.1 & 6.5 & 14 & $\sim 0.36$ & $\ldots$ & $1.6 \times 10^{33}$ & {$[2]$} \\
2XMMi J115836.1-623516 & G298.8-0.3 & 10 & 9.6 & $\ldots$ & $\ldots$ & $1.0 \times 10^{33}$ & {$[3]$} \\
Esta fuente & G290.1-0.8 & $\mathbf{1 0 - 2 0}$ & $\mathbf{7}$ & $\mathbf{0 . 2 3}_{-\mathbf{0 . 0 6}}^{+\mathbf{0 . 1 1}}$ & $\mathbf{0 . 7}$ & $\lesssim \mathbf{3} \times \mathbf{1 0}^{\mathbf{3 2}}$ & \\
\hline
\end{tabular}

Ref.: [1] Halpern \& Gotthelf (2010); [2] Combi et al. (2010); [3] Sánchez-Ayaso et al. (2012).

\subsection{Discusión}

La morfología peculiar observada en G290.1-0.8 podría ser el resultado de dos posibles escenarios físicos (o una combinación de ambos): i) una explosión esférica en un medio circumestelar e interestelar altamente inhomogéneo, o ii) la presencia de un objeto compacto central no detectado que genera estructuras tipo-jet en dos direcciones opuestas. El análisis espectral del SNR, mapa de MPE, y la información obtenida a partir de datos de radio, pueden dar pistas acerca de la naturaleza de la morfología radio/rayos X del SNR.

Nuestro análisis muestra que el remanente tiene una morfología con un pico de intensidad en el centro, más pronunciada en la banda blanda de rayos X, confirmando que pertenece a la clase de SNR con morfología mixta radio/rayos X. Además, del análisis espectral se desprende que existen abundancias moderadas de Si en todo el SNR y del S principalmente en la parte central, una situación que se observa también en otros SNRs del mismo tipo (ver, por ejemplo, Bocchino et al., 2009).

Teniendo en cuenta las temperaturas características, escalas de tiempo de ionización y flujos de rayos $\mathrm{X}$ obtenidos a partir del análisis espectral, podemos delinear un escenario astrofísico que permita explicar las características de todas las emisiones detectadas. La abrupta caída en el brillo superficial de rayos $\mathrm{X}$ en la dirección Noreste/Sudoeste, junto con las bajas temperaturas y grandes escalas temporales de ionización inferidas del análisis espectral, indican que en estos extremos el frente de choque del SNR ha alcanzado una región donde existen nubes moleculares 
y atómicas de alta densidad como reportaran Filipovic et al. (2005). Como resultado, el frente de choque original se divide en el choque inverso (shock reverse) y choque frontal (forward shock), los cuales se propagan en direcciones opuestas en ambos medios. Mientras el choque frontal es invisible en los rayos $\mathrm{X}$, el choque inverso es brillante y claramente menos energético que el choque original. El material en el choque inverso se enfría, dando lugar al equilibrio de ionización. Debido a la presencia de este choque inverso, el material eyectado brilla en la parte central del SNR, como lo demuestra Zhou et al. (2011). Por el contrario, en la dirección Noroeste/Sureste, debido a la presencia de una cavidad de baja densidad preexistente en el ISM con forma tubular, el frente de choque inicial se propaga más fácilmente sobre distancias más grandes, conservando su energía original. Por lo tanto, en las regiones NW y SE el plasma aún presenta altas temperaturas y permanece lejos del equilibrio de ionización. En este sentido, la morfología radio/rayos X del plasma emisor y sus propiedades espectrales pueden explicarse en términos de la distribución de la densidad del ISM preexistente en los alrededores del SNR.

Después de ajustar los espectros mediante diversos modelos, concluimos que no hay una contribución significativa de una componente no térmica a la emisión del SNR. El plasma térmico se encuentra a diferentes temperaturas y estados de ionización a lo largo del remanente, con abundancias químicas del plasma que corresponden a material eyectado en la explosión de la supernova que dio origen al SNR. Además, con el propósito de buscar alguna contribución del ISM a la emisión de rayos X en la región central brillante $(\mathrm{CT})$, se estudió como el ajuste espectral se modifica al incluir una segunda componente térmica con abundancia solar en el modelo, el cual no mejoró el ajuste. Los datos fueron bien ajustados con un modelo térmico de una sola temperatura, lo que sugiere que ninguna de las contribuciones del ISM es importante en todo el SNR.

Con el fin de hacer una comparación cuantitativa de nuestros resultados con los obtenidos por Slane et al. (2002) usando datos de $A S C A$, en el panel izquierdo de la Figura 4.7, se presentan los perfiles radiales de temperatura del SNR, derivados de nuestro análisis espectral (ver Tabla 4.2). Además, en el panel derecho, también mostramos los perfiles de brillo superficial normalizados, que resultan del conteo del número de fotones detectados por las cámaras MOS1/2. En ambos casos, el origen de los perfiles radiales se fijó en el centro geométrico del SNR. Después de la normalización, tanto para ASCA como XMM-Newton los perfiles de brillo superficial muestran la misma tendencia, aunque nuestros datos están siempre por debajo del perfil de $A S C A$. Por el contrario, nuestro perfil de temperatura es mayor que el perfil derivado de los datos $A S C A$ a lo largo de todo el SNR. En particular, las diferencias más grandes ocurren en las regiones más exteriores, donde el material expulsado, fuera del equilibrio de ionización, presenta temperaturas notablemente más altas que el resto del remanente. Sugerimos que este resultado es consecuencia de las condiciones de contorno impuestas por el ambiente en el que el remanente ha evolucionado, el cual se compone de una cavidad alargada pre-existente en dirección Noroeste-Sudeste, y dos regiones densas hacia los lados opuestos. Estas condiciones que introducimos como trazadores fundamentales de la morfología observada, no se han tenido en cuenta en el modelo propuesto por Slane et al. (2002), razón por la cual surgen las discrepancias con los escenarios teóricos que ellos analizan.

A pesar de que en el análisis realizado por Slane et al. (2002), el modelo asociado a pequeñas 

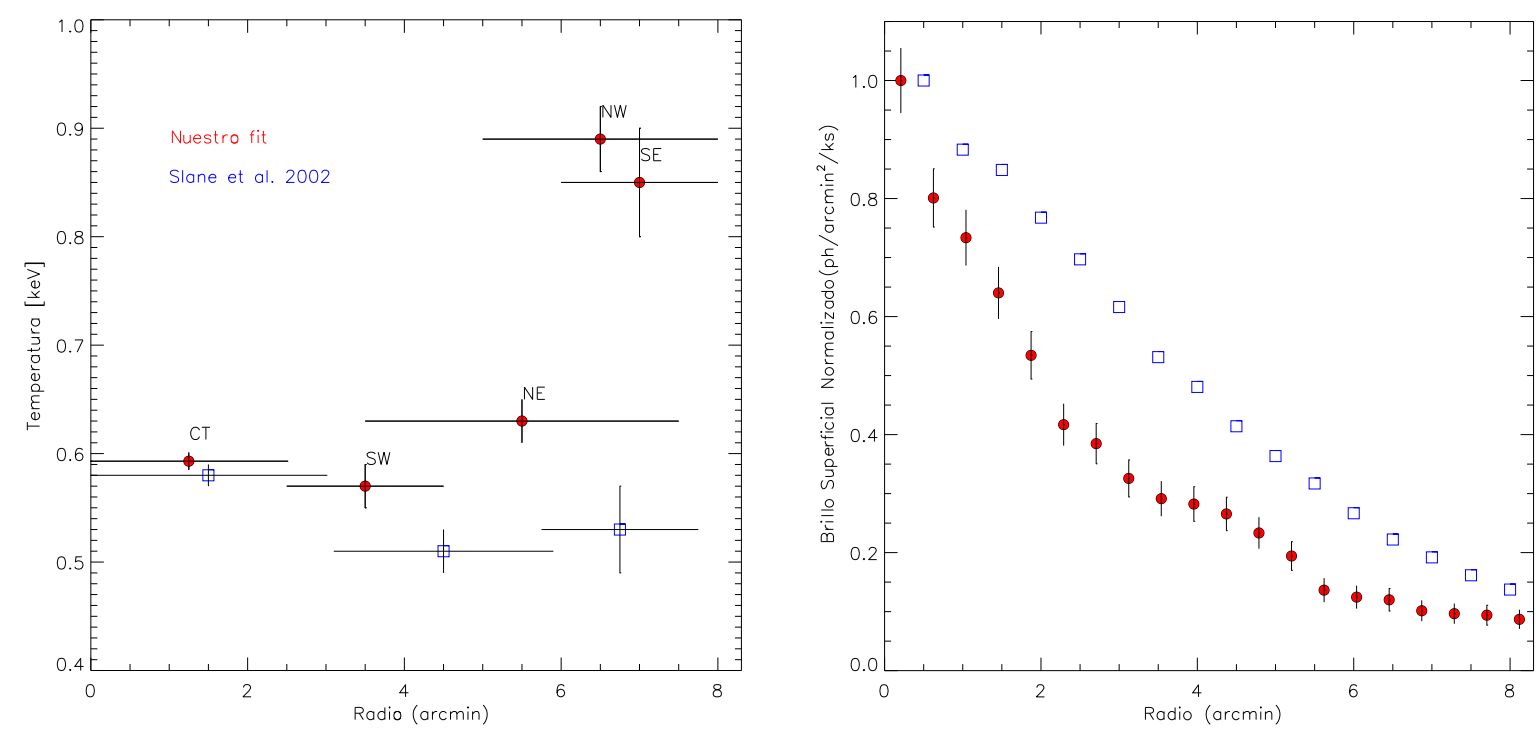

Figura 4.7: Panel izquierdo: Comparación de la distribución de temperaturas derivada de nuestro análisis espectral con el perfil radial de Slane et al. (2002). Panel derecho: Ídem para los perfiles de brillo superficial.

nubes de emisión (White \& Long, 1991) es la mejor opción para explicar las observaciones, encontraron un tiempo de evaporación de las nubes mayor que la edad deducida para el remanente, y un perfil de rayos $\mathrm{X}$ más empinado de lo esperado. Además, el modelo de nubes representa una situación en la que el remanente se expande en un medio uniforme. Nuestro análisis muestra claramente que este no es el caso y además, este modelo no puede explicar la presencia de material eyectado en la región central, que fuera claramente detectado en nuestro análisis espectral. Por lo tanto, una explicación para la morfología de la región brillante central en términos del resultado de un choque reflejado por la nubes densas en el medio circundante, que comprime el material eyectado en el centro (ver, por ejemplo, Zhou et al., 2011), parece mucho más plausible que el de evaporación propuesto por Slane et al. (2002).

Con el fin de desentrañar este punto, una comparación cuantitativa de los resultados aquí obtenidos con el modelo propuesto por Zhou et al. (2011) será de gran ayuda. Sin embargo, sus simulaciones fueron ajustadas para reproducir la morfología del SNR W49B, y el desarrollo de un modelo numérico completo para G290.1-0.8 se aleja del propósito de nuestro estudio.

El hecho de que a lo largo de todo el SNR la abundancia del Fe es baja $(\lesssim 0.1)$ favorece fuertemente una explosión de supernova originada como resultado del colapso gravitacional de una estrella de gran masa. Esto también se confirma mediante una comparación entre los cocientes de abundancias del Mg/Si, S/Si y Fe/Si (0.5, 0.8 y 0.05 respectivamente, en la región central) que son ampliamente consistentes con modelos con masas de 25-30 $\mathrm{M}_{\odot}$ (Woosley \& Weaver, 1995) y fuertemente incompatibles con cualquier escenario degenerado (ver, por ejemplo, la Tabla 3 de Troja et al., 2008, y sus referencias). En este caso, un fuerte viento bipolar de la estrella progenitora podría ser el responsable de la estructura alargada del medio, debido a que las estrellas de gran 
masa pueden depositar $\sim 10^{51}$ erg de energía cinética a su entorno a través de un viento asimétrico (Lamers \& Cassinelli, 1999).

\subsection{Conclusiones}

En este trabajo se han analizado datos radio, de rayos X y del H I del SNR G290.1-0.8 con el objeto de investigar el origen de la morfología peculiar observada en frecuencias de radio/rayos X, como también la interacción de la fuente con el medio interestelar circundante y realizar una búsqueda de un remanente compacto en su interior. La información reunida apoya un escenario astrofísico en el que las morfologías de radio y de rayos X son el resultado de una explosión esférica en un medio de alta densidad que presenta una cavidad de baja densidad preexistente con una estructura tubular en la dirección en Noroeste/Sudeste.

$\mathrm{El}$ análisis espectral de rayos X y el mapa de energía media de fotones obtenido confirman que las condiciones físicas del plasma son fuertemente inhomogéneas a lo largo del remanente. $\mathrm{Si}$ bien la emisión de rayos X en las regiones CT, NE y SW han alcanzado el equilibrio de ionización, en las zonas de NW y SE el plasma emisor permanece fuera del equilibrio. Por otra parte, las temperaturas en las partes CT, SW y NE son significativamente inferiores a las obtenidas para las regiones NW y SE. Las abundancias químicas también son diferentes, y en todos los casos corresponden a material eyectado y no al medio preexistente donde el SNR se expande.

Basado en los resultados del análisis de rayos $\mathrm{X}$, se sugiere que el origen de la morfología observada involucra una supernova producto del colapso de una estrella de gran masa, si la acción de un medio circundante pre-existente es tomado en cuenta. En este caso, un fuerte viento bipolar de la estrella progenitora podría ser el responsable de la cavidad alargada en forma de tubo observada a frecuencias de radio.

Aunque la evidencia aquí obtenida da apoyo a que el SNR es el resultado de una explosión esférica en un medio de alta densidad anisotrópico, la posibilidad de que un objeto compacto, como una NS, se encuentre dentro del SNR, no puede descartarse. Observaciones profundas realizadas con Chandra podrían ayudar a revelar más detalles de la emisión de rayos X y discernir la presencia de una fuente de rayos $\mathrm{X}$ puntual compacta dentro del remanente. 


\section{Capítulo 5}

\section{El enfriamiento de la estrella de neutrones en el sistema binario 4U 1820-30}

\subsection{Resumen}

En este capítulo presentamos el análisis del conjunto completo de erupciones de rayos $\mathrm{X}$ de tipo-I de la NS en el sistema binario de baja masa 4U 1820-30 detectadas con el satélite RXTE. Mostramos aquí que todas estas erupciones son debidas a la rápida expansión y compresión del radio de su fotósfera, teniendo todas ellas duraciones, flujos máximos y fluencias comparables.

Del análisis de la evolución temporal de sus espectros, en escalas de decenas de segundos, encontramos que la relación entre el flujo bolométrico y la temperatura es muy diferente de la relación canónica $F \propto T^{4}$, esperable en el caso en que el área aparente de emisión en la superficie de la NS permanece constante. Por el contrario, la relación flujo-temperatura observada puede ajustarse más adecuadamente mediante una ley de potencias quebrada con un primer índice $v_{1}=2.0 \pm 0.3$ y un segundo índice $v_{2}=5.76 \pm 0.06$.

El desvío respecto a la relación con índice $v=4$ durante la fase de enfriamiento de dichas erupciones de rayos X en 4U 1820-30 podría deberse a cambios en el área de emisión de la estrella mientras que su atmósfera se enfría, a variaciones en el factor de corrección de color producto de cierta evolución química, o a la presencia de una fuente de calor remanente, como una quema nuclear residual de hidrógeno acretado, que podría jugar un rol importante mientras la emisión de la erupción cesa.

En todos los casos, los resultados de este trabajo tienen fuertes consecuencias sobre la determinación del radio de las NSs en estrellas binarias de baja masa. 


\subsection{Introducción}

La acreción de NSs en LMXBs produce episodios de quema termonuclear inestable de $\mathrm{H}$ y He en su superficies (ver, por ejemplo, Galloway et al., 2008; Lewin et al., 1993; Strohmayer \& Bildsten, 2006). Estos episodios se denominan erupciones de rayos X de tipo-I. Aunque la gran mayoría de la energía liberada en los LMXBs se origina en el proceso de acreción, por el cual resultan capaces de irradiar $\sim 300 \mathrm{MeV}$ por barión acretado, el proceso de fusión inestable ( $\lesssim 5 \mathrm{MeV}$ por nucleón) llevado a cabo durante una erupción de rayos $\mathrm{X}$ de tipo-I puede aumentar la luminosidad de estos objetos en un factor $\sim 10$ en tan solo unos $0.5-5 \mathrm{~s}$. Después de este ascenso rápido, el flujo de rayos X disminuye de nuevo de manera exponencial dentro de los 10-100 s. La energía total típica que es emitida durante una erupción de rayos $\mathrm{X}$ es $\sim 10^{39} \mathrm{erg}$. Algunas de estas explosiones son lo suficientemente fuertes como para alcanzar la luminosidad de Eddington. En esos casos, la presión de radiación es lo suficientemente alta como para provocar la expansión de las capas exteriores de la atmósfera de la estrella, dando lugar a las erupciones con expansión de radio fotosférico ( $\mathrm{PRE}^{1}$, Basinska et al., 1984). La hipótesis de que estas explosiones se originan en la superficie de la estrella es apoyada por el hecho de que el área de emisión inferida durante las explosiones coincide con el área de la superficie aparente de una estrella estándar $\left(\sim 400 \mathrm{~km}^{2}\right)$, si se asume una fase de enfriamiento asimétrica (Bildsten, 1995; Fryxell \& Woosley, 1982; Spitkovsky et al., 2002; Strohmayer \& Bildsten, 2006). Este área de emisión puede estimarse a partir de un análisis espectral considerando su evolución temporal durante toda la fase de enfriamiento posterior a estas erupciones.

La fuente de rayos X 4U 1820-30 es una binaria de rayos X ultra-compacta, con un período orbital de 11.4 min (Stella et al., 1987), formada por una NS y una enana blanca de 0.06-0.08 $\mathrm{M}_{\odot}$ que ha consumido todo su hidrógeno (Rappaport et al., 1987). 4U 1820-30 pertenece al cúmulo globular NGC 6624, localizado a 7.6 $0.4 \mathrm{kpc}$ de distancia (Kuulkers et al., 2003). El sistema ha presentado oscilaciones cuasi-periódicas que fueron reportadas por Smale et al. (1997) y Zhang et al. (1998). Las erupciones de rayos X tipo-I en esta LMXB fueron descubiertas por Grindlay et al. (1976). Hasta junio de 2007, cinco erupciones de rayos X de tipo-I -todas PRE- fueron detectadas a través de RXTE (Galloway et al., 2008). Usando tres de estas cinco erupciones, Güver et al. (2010) señalaron que durante la fase de enfriamiento el flujo bolométrico es proporcional a la temperatura elevada a la cuarta potencia, a partir de lo cual estimaron la masa y radio de $4 \mathrm{U}$ 1820-30 con gran precisión. Strohmayer \& Brown (2002) también descubrió una super-erupción ${ }^{2}$ en esta fuente, presumiblemente debido a la quema de una capa profunda de C. Las características de estas erupciones de rayos X, fueron modeladas por Cumming (2003) considerando una NS acretando material enriquecido en He.

Presentamos aquí el estudio de la muestra completa de las explosiones de rayos $\mathrm{X}$ detectadas con $R X T E$ en 4 U 1820-30. Exploramos las propiedades de estas explosiones focalizándonos en el estudio de su comportamiento durante la fase del enfriamiento, que permite obtener elementos cruciales para poder determinar la masa y el radio de la NS y deducir así propiedades de la

\footnotetext{
${ }^{1}$ del inglés, Photospheric Radius Expansion bursts

${ }^{2}$ conocida como superburst en inglés
} 
EoS en su interior. Comparamos además nuestros resultados con los obtenidos por Zhang et al. (2011) para la fase de enfriamiento de 4U 1636-56, y con modelos recientes de atmósferas de NSs desarrollados por Suleimanov et al. (2011).

\subsection{Observaciones y análisis de datos}

Analizamos todos los datos de archivo de 4U 1820-30 obtenidos con el instrumento Proportional Counter Array (PCA) del satélite RXTE. Las observaciones abarcan el período que va desde mayo de 1996 hasta abril de 2011. El detector PCA consiste de 5 unidades de contadores proporcionales (PCUs) sensibles en el rango de energía de 2 a $60 \mathrm{keV}$. Con el fin de detectar estallidos de rayos $\mathrm{X}$ en la base de datos generamos curvas de luz con resolución temporal de $1 \mathrm{~s}$ utilizando el modo estándar-1 (datos empaquetados cada $0.125 \mathrm{~s}$ sin resolución en energía), y hemos analizado cada curva de luz en busca de tres puntos consecutivos a 8- $\sigma$ por encima de la tasa de cuentas promedio de las observaciones, valor que elegimos para distinguir los estallidos reales de las fluctuaciones características de la emisión persistente. Confirmamos además la detección de los estallidos inspeccionando a ojo las curva de luz de toda la muestra. Siguiendo el procedimiento descripto en Zhang et al. (2009), obtuvimos los colores de rayos X a partir de los datos del modo estándar-2 (resolución temporal de 16 s y 129 canales de energía). Para ello seleccionamos cuatro bandas de energía: $A=2.0-3.5 \mathrm{keV}, B=3.5-6.0 \mathrm{keV}, C=6.0-9.7 \mathrm{keV}$ y $D=9.7-16.0 \mathrm{keV}$, y definimos colores blandos y duros como cocientes de cuentas $S C=B / A$ y $H C=D / C$, respectivamente. También definimos la intensidad de rayos $\mathrm{X}, I_{\mathrm{X}}$, como el número de cuentas en el rango 2.0-16.0 keV. Los cocientes de cuentas en cada rango de energía fueron normalizados usando los datos de PCA del "Crab" obtenidos cerca del momento de cada observación, del modo usual.

Para estudiar en detalle cada erupción de rayos X, usamos los datos de PCA en el modo de evento, E_125us_64M_0_1s, donde cada dato está combinado en 64 canales de energía en el rango de 2-60 keV, con una resolución temporal de 125- $\mu$ s. Para el análisis de la evolución temporal, extrajimos espectros usando los 64 canales de energía combinando los datos a intervalos de tiempo que van desde $0.125 \mathrm{~s}$ en el pico a 1-2 s en la cola de la erupción.

Corregimos el tiempo de exposición de cada espectro por tiempos muertos ${ }^{3}$ utilizando los métodos proporcionados por el equipo ${ }^{4}$ del $R X T E$ y generamos matrices de respuesta para las unidades de PCA activas durante cada observación. Los datos fueron ajustados utilizando la versión 12.7.0 de XSPEC (Arnaud, 1996) asumiendo un error sistemático del 5 por ciento, como sugiere el grupo de calibración ${ }^{5}$ de $R X T E$. Los espectros producidos (250 en total) fueron ajustados en el rango de 2.5 a $20 \mathrm{keV}$. Un detalle en el esquema de combinación de los canales de los archivos de eventos lleva a obtener un canal con cero cuentas en una de las PCUs (Jahoda et al., 1996). Si bien esto es tenido en cuenta por la matriz de respuesta, encontramos, sin embargo, que si en los casos en que sólo una PCU estuvo disponible durante la observación, ese canal con cero

\footnotetext{
${ }^{3}$ del inglés, deadtime corrections

${ }^{4}$ http://heasarc.gsfc.nasa.gov/docs/xte/whatsnew/deadtime.html

${ }^{5}$ http://www . universe.nasa.gov/xrays/programs/rxte/pca/doc/rmf/pcarmf-11.7/
} 
cuentas afecta el ajuste espectral. En nuestro caso, esto ocurrió en unos pocos espectros en los cuales sólo estaba una PCU disponible, y el canal 11 tenía cero cuentas. Por esta razón, ignoramos el canal $11(E \sim 4 \mathrm{keV})$ en todos los espectros, obteniendo finalmente 25 canales en el rango de 2.5-20 keV para cada espectro.

Para el fondo, extrajimos $\sim 100$ s de espectro de la emisión persistente antes y después de cada erupción, siguiendo el procedimiento estándar en el análisis de estallidos de rayos X (ver, por ejemplo, Kuulkers et al., 2002). Ajustamos los espectros usando un modelo de cuerpo negro (bbodyrad) y un modelo de absorción interestelar (tbabs), fijando la columna de hidrógeno, $N_{\mathrm{H}}$. El modelo para la absorción usa las abundancias de Wilms et al. (2000) y las secciones eficaces de Verner et al. (1996). Así, el modelo espectral consta de dos parámetros libres: la temperatura del cuerpo negro, $k T_{\mathrm{bb}}$, en unidades de $\mathrm{keV}$ (donde $k$ es la constante de Boltzmann) y la normalización del cuerpo negro, $N_{\mathrm{bb}}=\left(R_{\mathrm{bb}} / d_{10}\right)^{2}$, donde $R_{\mathrm{bb}}$ es el radio del cuerpo negro en km y $d_{10}$ es la distancia a la fuente en unidades de $10 \mathrm{kpc}$. Estos parámetros nos permiten estimar la evolución del flujo bolométrico durante el estallido usando, por ejemplo, la Ecuación 3 de Galloway et al. (2008), $F=0.001076 N_{\mathrm{bb}}\left(k T_{\mathrm{bb}}\right)^{4} \quad 10^{-8} \mathrm{erg} \mathrm{cm}^{-2} \mathrm{~s}^{-2}$

Este procedimiento falla si el cuerpo negro observado durante el estallido proviene del mismo cuerpo negro que produce la emisión persistente antes del estallido, porque la diferencia entre dos espectros de cuerpo negro no es un cuerpo negro (van Paradijs \& Lewin, 1986). Sin embargo, en la práctica, las desviaciones de una distribución Planckiana se vuelven importantes sólo si la emisión neta es débil, es decir, al comienzo o en la cola de los estallidos únicamente.

Para probar si el uso de los parámetros del ajuste del espectro neto en lugar del espectro total -que incluye el modelado de la emisión persistente durante la erupción restando el fondo instrumental- podrían afectar los resultados finales, procedimos de la siguiente manera: ajustamos el espectro persistente antes de cada estallido utilizando un cuerpo negro absorbido más un modelo de ley de potencia. Luego, durante el tiempo que duró la erupción, fijamos el índice de la ley de potencias y la normalización a los valores del mejor ajuste obtenidos para la emisión persistente y ajustamos solamente los parámetros del cuerpo negro. Los resultados de este procedimiento fueron consistentes dentro de los errores con los obtenidos ajustando el espectro neto, ya sea utilizando la emisión persistente antes o después de cada erupción como fondo. Para estudiar si variaciones posibles en la emisión persistente durante el tiempo de la erupción podrían afectar nuestros resultados, también ajustamos los espectros dejando libre la componente ley de potencias. Los resultados de este análisis también fueron totalmente consistentes dentro de los errores con los encontrados mediante el ajuste de los espectros netos. También evaluamos la contribución de la emisión persistente a los espectros netos durante el tiempo del estallidos; y encontramos que para cada espectro, la contribución del cuerpo negro es de al menos el 25 por ciento de la emisión total en la banda de energía de 2.5-20 keV, siendo en el 75 por ciento de los casos, mayor a la mitad. Analizados estos puntos, de aquí en adelante presentamos los resultados obtenidos mediante el procedimiento estándar.

En todos los espectros el número de cuentas por canal en la fuente y en el fondo es lo suficientemente alto como para justificar el uso de la estadística de $\chi^{2}$ (Pearson, 1900) para encontrar los parámetros del mejor ajuste. Por lo tanto, al contrario de Güver et al. (2010), no recombinamos 
Tabla 5.1: Estallidos de rayos X en 4U 1820-30 detectados con $R X T E$.

\begin{tabular}{clccccc}
\hline Observación ID & $\begin{array}{c}\text { Tiempo de inicio } \\
{[\mathrm{MJD}]}\end{array}$ & $\begin{array}{c}\text { Duración } \\
{[\mathrm{s}]}\end{array}$ & $\begin{array}{c}\mathrm{SC}^{*} \\
{[\mathrm{Crab}]}\end{array}$ & $\begin{array}{c}\mathrm{HC}^{*} \\
{[\mathrm{Crab}]}\end{array}$ & $\begin{array}{c}I_{\mathrm{X}}{ }^{*} \\
{[\mathrm{Crab}]}\end{array}$ \\
\hline$\dagger$ & $20075-01-05-00$ & 50570.73116038 & $\sim 26$ & $1.11(1)$ & $0.91(1)$ & $0.1263(3)$ \\
& $30057-01-04-08 \mathrm{G}$ & 51430.0461502 & $>3000{ }^{* *}$ & $1.08(4)$ & $0.80(4)$ & $0.112(1)$ \\
$\dagger$ & $40017-01-24-00$ & 52794.73816848 & $\sim 28$ & $1.19(2)$ & $1.00(1)$ & $0.0894(4)$ \\
$\dagger$ & $70030-03-04-01$ & 52802.07560482 & $\sim 26$ & $1.15(2)$ & $0.98(1)$ & $0.0914(4)$ \\
$\dagger$ & $70030-03-05-01$ & 52805.89569741 & $\sim 25$ & $1.19(1)$ & $1.01(1)$ & $0.1238(4)$ \\
$90027-01-03-05$ & 53277.43859093 & $\sim 23$ & $1.09(1)$ & $0.89(1)$ & $0.1317(5)$ \\
$94090-01-01-02$ & 54948.82125875 & $\sim 28$ & $1.15(1)$ & $0.98(1)$ & $0.1130(4)$ \\
$94090-01-01-05$ & 54950.70282704 & $\sim 30$ & $1.22(2)$ & $1.01(1)$ & $0.1012(4)$ \\
$94090-01-02-03$ & 54956.77474255 & $\sim 25$ & $1.26(2)$ & $1.09(2)$ & $0.0949(4)$ \\
$94090-01-02-02$ & 54958.73999718 & $\sim 31$ & $1.27(2)$ & $1.10(1)$ & $0.0912(4)$ \\
$94090-01-04-00$ & 54978.32150760 & $\sim 30$ & $1.33(2)$ & $1.11(2)$ & $0.1052(5)$ \\
$94090-01-04-01$ & 54978.49491038 & $\sim 29$ & $1.30(2)$ & $1.14(1)$ & $0.1048(5)$ \\
$94090-01-05-00$ & 54981.18730621 & $\sim 26$ & $1.29(2)$ & $1.12(1)$ & $0.1130(5)$ \\
$94090-02-01-00$ & 54994.53419278 & $\sim 21$ & $1.18(1)$ & $1.02(1)$ & $0.1855(6)$ \\
$94090-02-01-00$ & 54994.61302380 & $\sim 22$ & $1.19(1)$ & $0.99(1)$ & $0.1905(7)$ \\
$96090-01-01-00 G$ & 55624.88068005 & $\sim 25$ & $1.22(2)$ & $1.01(1)$ & $0.0940(4)$ \\
$96090-01-01-020$ & 55626.77361987 & $\sim 21$ & $1.11(1)$ & $0.83(1)$ & $0.1707(6)$ \\
\hline
\end{tabular}

* Colores de rayos X blandos/duros e intensidad de la emisión persistente justo antes del estallido de rayos $\mathrm{X}$, en unidades de Crab.

** Superburst, ver Strohmayer \& Brown (2002).

† Erupción de rayos X analizada por Güver et al. (2010).

los espectros en energía (notamos que dado el esquema de original de los datos, que están combinados con un sobre muestreo de la resolución espectral intrínseca de PCA por un factor de 1 a 3, su recombinación no es necesaria ni deseable). A modo de comparación, también ajustamos todos los espectros utilizando la estadística C definida por Cash (1979), para una estadística intrínsecamente poissoniana. Encontramos resultados consistentes con ambos métodos, y por lo tanto reportamos aquí los resultados obtenidos con $\chi^{2}$. En los gráficos mostramos errores a 1- $\sigma$ $\left(\Delta \chi^{2}=1\right)$.

\subsection{Resultados}

\subsubsection{Detección de erupciones de rayos $\mathrm{X}$ y análisis de diagramas de color}

De todos los datos de archivo de RXTE, se identificaron 16 erupciones cortas (20-30 s), y la presencia de una gran erupción (> 3000 s), previamente reportada por Strohmayer \& Brown 
(2002) y conocida como superburst. En la Tabla 5.1, se resumen las principales propiedades de las erupciones de rayos $\mathrm{X}$ detectadas. El momento de inicio de las erupciones se definió como un aumento continuo del cociente de cuentas PCA persistente por al menos $0.25 \mathrm{~s}$ dentro de cada erupción, mientras que el momento de finalización se definió como el tiempo cuando la tasa de cuentas retorna a menos de 3- $\sigma$ del flujo de la emisión persistente antes de su aparición. También se muestran en la tabla la duración -diferencia entre el tiempo final e inicial- los colores de rayos $\mathrm{X}$ blandos $(S C)$, duros $(H C)$ y la intensidad de la emisión persistente antes de cada estallido $\left(I_{\mathrm{X}}\right)$, derivados de los datos en modo estándar-2 de PCA.

Las Figuras 5.1 y 5.2, muestran la posición de la fuente en el diagrama color-color (CD) y dureza-intensidad (HID) antes de cada erupción de rayos X, respectivamente. Notar que ninguna de las erupciones fue detectada para $H C<0.8$ ni para $I_{\mathrm{X}}>0.2$ Crab, que corresponden a la fuente en estado intenso y blando, para el cual la tasa de acreción probablemente sea muy alta (Hasinger \& van der Klis, 1989).

\subsubsection{Análisis espectral de los estallidos de rayos $\mathrm{X}$}

En la Figura 5.3 se muestran los resultados de los ajustes de una de las erupciones de rayos X detectadas en 4 U 1820-30. Debido a que todas las erupciones son muy similares, mostramos aquí una de ellas a modo de ejemplo para ilustrar sus características. En el panel superior se presenta la curva de luz en modo estándar-1 de alta resolución temporal, comenzando $1 \mathrm{~s}$ antes del inicio de la erupción. Al comenzar la erupción, la curva de luz muestra un pico muy alto y estrecho que dura 1-2 s. Luego, la curva comienza a crecer otra vez alcanzando un máximo de $\sim 5000$ cuenta s $^{-1}$ en 2-3 s, y finalmente decae exponencialmente hasta que la curva vuelve a la emisión persistente en 15-25 s. En los cuatro paneles restantes se presenta la evolución de los parámetros del mejor ajuste obtenido, $k T_{\mathrm{bb}}$ y $N_{\mathrm{bb}}$, el flujo bolométrico derivado del modelo de cuerpo negro y los residuos de $\chi^{2}$ reducido correspondientes a 23 grados de libertad, respectivamente.

La distribución $\chi^{2}$ del mejor ajuste de los 250 espectros es consistente con la distribución $\chi^{2}$ esperada. El test de Kolmogorov-Smirnov entre las dos distribuciones da una probabilidad de 0.09 , insuficiente para refutar la hipótesis de que el mejor ajuste de $\chi^{2}$ pueda derivarse de la distribución $\chi^{2}$ esperada para 23 grados de libertad. Durante el ajuste espectral, consideramos dos valores diferentes para la densidad de la columna de H, siguiendo Costantini et al. (2012) y Güver et al. (2010): $N_{\mathrm{H}}=0.16$ y $0.25 \times 10^{22} \mathrm{~cm}^{-2}$, respectivamente. Los parámetros espectrales del mejor ajuste obtenidos para estas densidades columnares son plenamente consistentes entre sí, debido a que en la banda de energía $2.5-20 \mathrm{keV}$, la absorción no es muy significativa. A partir de aquí, mostramos los resultados obtenidos asumiendo $N_{\mathrm{H}}=0.25 \times 10^{22} \mathrm{~cm}^{-2}$.

$\mathrm{Al}$ comienzo de la erupción de rayos $\mathrm{X}$ la temperatura de cuerpo negro, $k T_{\mathrm{bb}}$, arranca en $\sim 3 \mathrm{keV}$ y disminuye rápidamente, mientras que la normalización, $N_{\mathrm{bb}}$, que es proporcional al área de emisión inferida, aumenta rápidamente. Luego, $k T_{\mathrm{bb}}$ comienza a aumentar de nuevo cuando $N_{\mathrm{bb}}$ decrece, hasta que alcanza el punto de contacto. En este punto, el $N_{\mathrm{bb}}$ es mínimo y la temperatura es máxima. Por lo tanto, definimos este momento como el inicio de la fase de enfriamiento. Este comportamiento es común a todas las erupciones, e indica que todas ellas son tipo PRE. El pico 


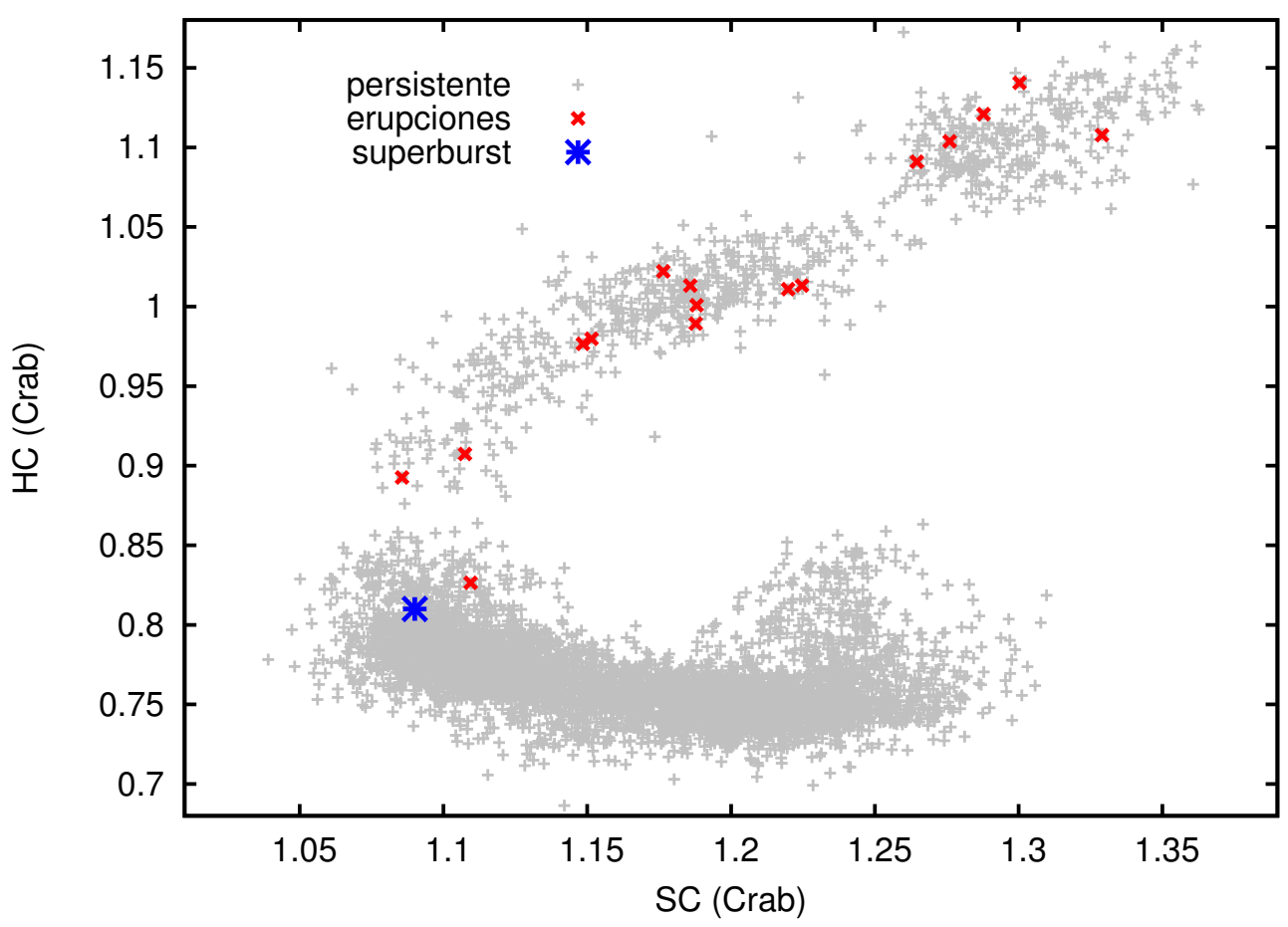

Figura 5.1: Diagrama color-color de todas las observaciones $R X T E$ de $4 \mathrm{U}$ 1820-30. Los puntos grises corresponden a todo el grupo de observaciones a una resolución temporal de $256 \mathrm{~s}$. El color de la emisión persistente previa a los 16 estallidos de rayos X se indica con cruces rojas. El color de la emisión persistente previa al superburst se indica con una estrella azul. Los colores están dados en unidades del Crab.

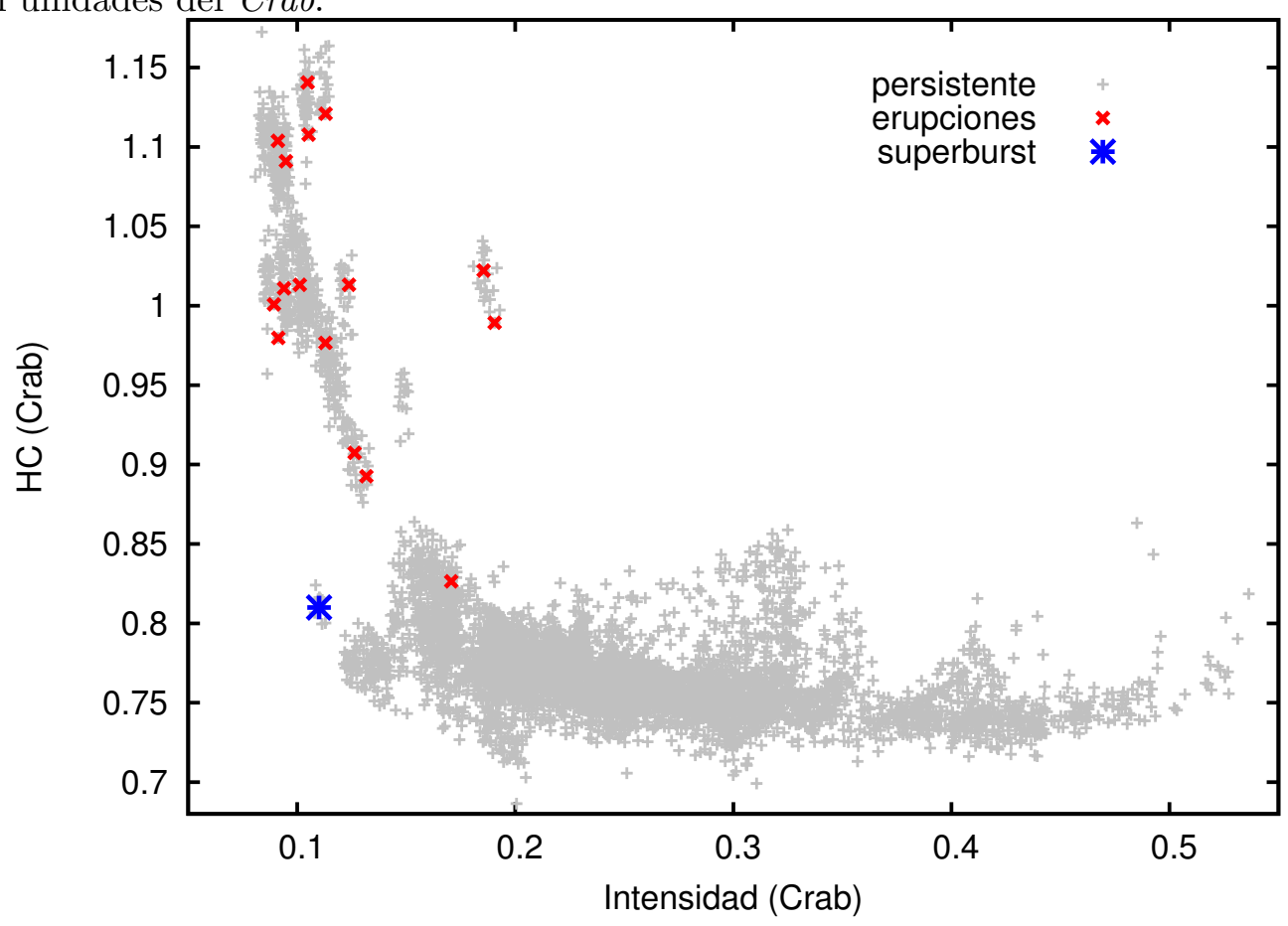

Figura 5.2: Diagrama de dureza-intensidad de todas las observaciones $R X T E$ de $4 \mathrm{U}$ 1820-30. Los símbolos son los mismos que en la Figura 5.1. La intensidad y color están en unidades del Crab. 


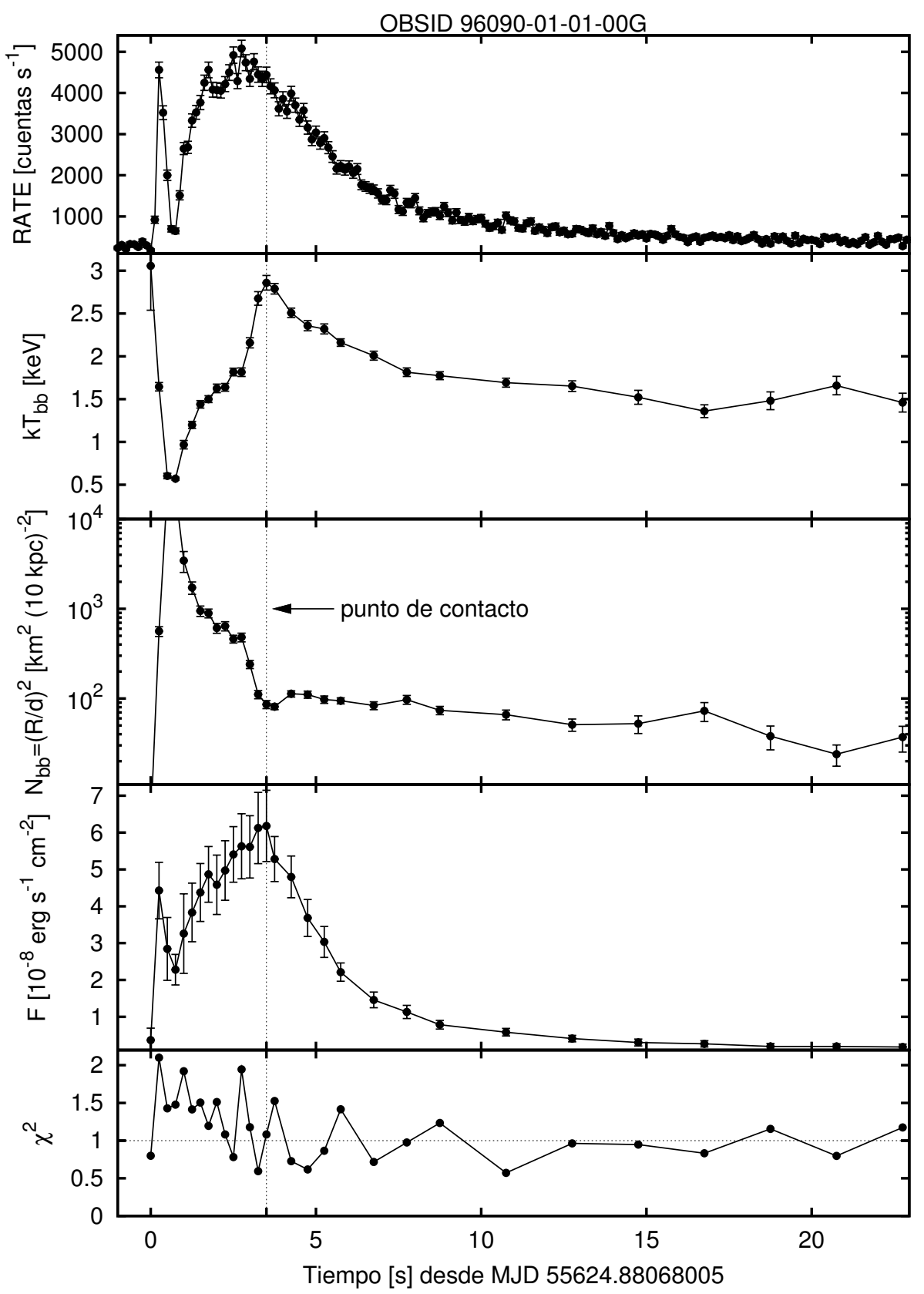

Figura 5.3: Erupción de rayos X en 4U 1820-30 detectada en la ObsID 96090-01-01-00G. Los paneles de arriba y abajo corresponden a la curva de luz del modo estándar-1, con resolución temporal de $0.125 \mathrm{~s}$, comenzando $1 \mathrm{~s}$ antes de la detección de la erupción, la temperatura del cuerpo negro, $k T_{\mathrm{bb}}$, normalización, $N_{\mathrm{bb}}$, flujo bolométrico, $F$, y $\chi^{2}$ reducido para 23 grados de libertad del mejor ajuste del espectro (comenzando desde el inicio del estallido) con barras de error a 1- $\sigma$. La línea vertical punteada indica el punto de contacto, que define el inicio de la fase de enfriamiento. 
alto y estrecho observado en la curva de luz al comienzo de cada erupción (Keek, 2012) se debe al hecho de que la temperatura disminuye lo suficiente como para mover la distribución de flujo planckiana a la banda de rayos X blanda, fuera del rango de energía donde PCA es sensible (in't Zand \& Weinberg, 2010; Tawara, Hayakawa, \& Kii, 1984).

Con el fin de estudiar las propiedades de toda la muestra de erupciones, analizamos el comportamiento de los cuatro parámetros inferidos a partir del análisis de rayos X con respecto a la posición de la erupción en el CD. Estos parámetros son: la temperatura, $k T_{\text {pico }}$, y flujo, $F_{\text {pico }}$, en el punto de contacto, la fluencia, $B$, definida como la energía total por unidad de área emitida durante el estallido $B=\int F(t) d t$, y la escala de tiempo del decaimiento, $\tau$, obtenida a partir del ajuste de una función exponencial al flujo durante la fase de enfriamiento de cada erupción. Dividimos los estallidos en dos grupos en base al $H C$ de la emisión persistente en el momento del estallido: $\mathrm{HC}>1.05$ y $\mathrm{HC}<1.05$. Del análisis estadístico de las dos submuestras vemos que ambas poblaciones son indistinguibles por estos parámetros (ver también in’t Zand et al., 2012).

\subsubsection{Relación flujo-temperatura durante la fase de enfriamiento}

En la Figura 5.4 exploramos la relación entre el flujo bolométrico, $F$, y la temperatura del cuerpo negro, $k T_{\mathrm{bb}}$, durante la fase de enfriamiento de todas las erupciones. Las líneas diagonales en el gráfico representan líneas de radio constante, $R=4,6$ y $8 \mathrm{~km}$, respectivamente, suponiendo una distancia $d=7.6 \mathrm{kpc}$ a la fuente. A pesar que la dispersión es alta a bajos niveles de flujo, todas los estallidos siguen la misma tendencia en este diagrama (la dispersión en el gráfico es consistente con los errores estadísticos del flujo y la temperatura). Dado que la relación flujotemperatura de todas las erupciones es consistente con ser la misma, combinamos los datos a un total de 25 puntos, calculando el promedio pesado del flujo y la temperatura. También utilizamos otros factores de agrupamiento, pero en todos los casos obtuvimos resultados consistentes.

En la Figura 5.5 graficamos la relación flujo-temperatura para los datos combinados. En este gráfico vemos que la fase de enfriamiento del estallido de rayos X en 4U 1820-30 no sigue la relación esperada $F \propto\left(k T_{\mathrm{bb}}\right)^{4}$ para un cuerpo negro ideal con área de emisión constante. Ajustando los datos con una ley de potencias simple, $F \propto\left(k T_{\mathrm{bb}}\right)^{v}$, resulta en $v=4.95 \pm 0.03$, pero el ajuste no es bueno ya que $\chi^{2}=499$ para 23 grados de libertad. En cambio, una ley de potencias quebrada ajusta mejor los datos (aunque formalmente no sea un buen ajuste) con $\chi^{2}=44$ para 21 grados de libertad, dando como resultado índices de ley de potencia antes y después del quiebre $v_{1}=2.0 \pm 0.3$ y $v_{2}=5.72 \pm 0.06$, respectivamente, y un quiebre a $k T_{\mathrm{bb}}=2.43 \pm 0.02 \mathrm{keV}$ (ver Figura 5.5).

También estudiamos la relación de flujo-temperatura dividiendo los estallidos en dos grupos para $H C>1.05$ y $H C<1.05$, como en las secciones anteriores. Después de combinar ambos conjuntos de datos en 25 puntos, graficamos sus respectivas fases de enfriamiento y confirmamos que ambas curvas son consistentes dentro de los errores, lo cual apoya la idea de que la muestra completa de erupciones de rayos X tipo-I en 4U 1820-30 es homogénea. 


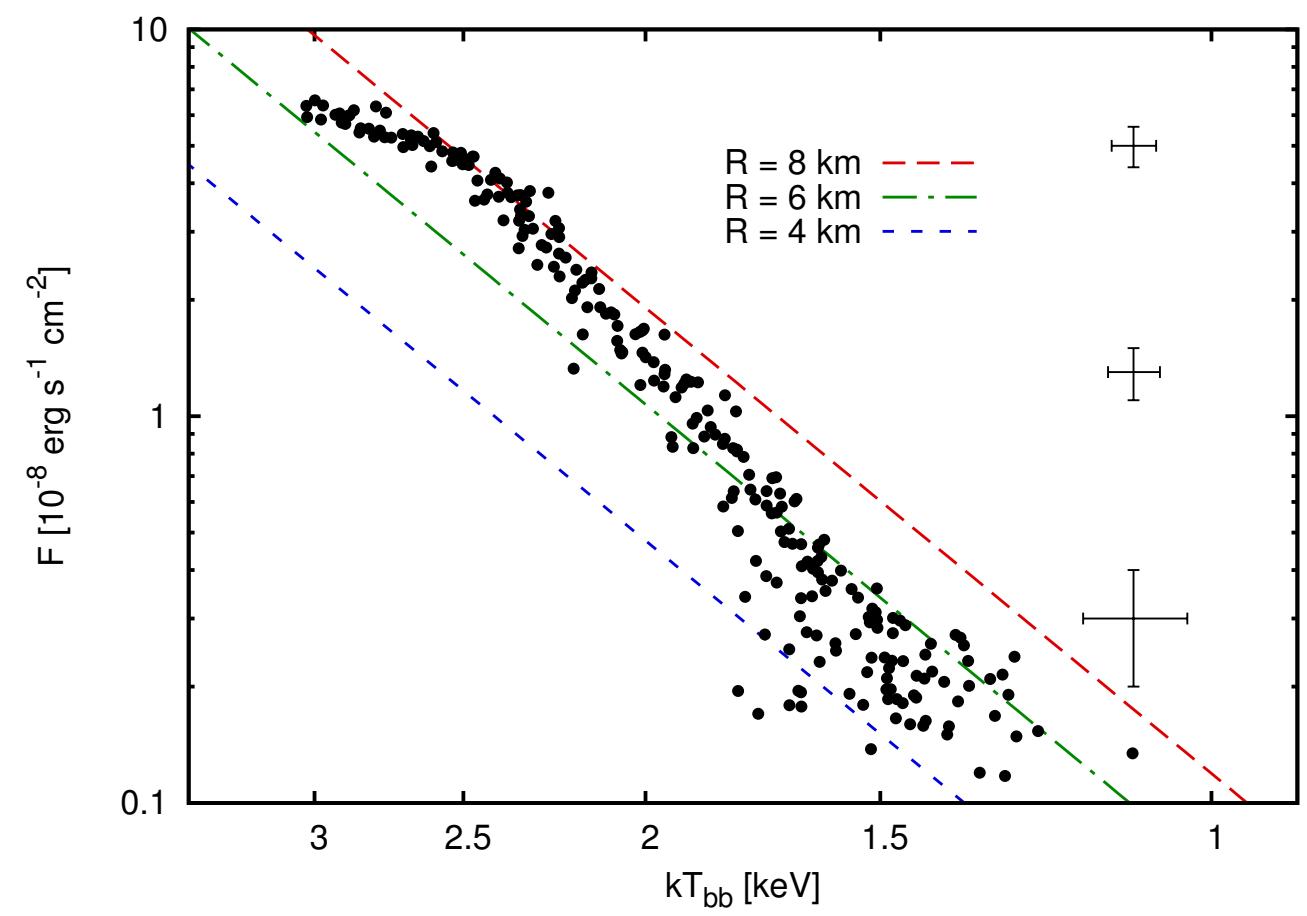

Figura 5.4: Fase de enfriamiento de las 16 erupciones de rayos X de $4 \mathrm{U}$ 1820-30. Las tres diagonales son líneas de radio constante, correspondientes, respectivamente, a tres cuerpos negros de $R=4$, 6 y $8 \mathrm{~km}$, asumiendo una distancia de $7.6 \mathrm{kpc}$ a la fuente. En la parte derecha del gráfico se muestran las barras de error típicas para los tres niveles de flujo.

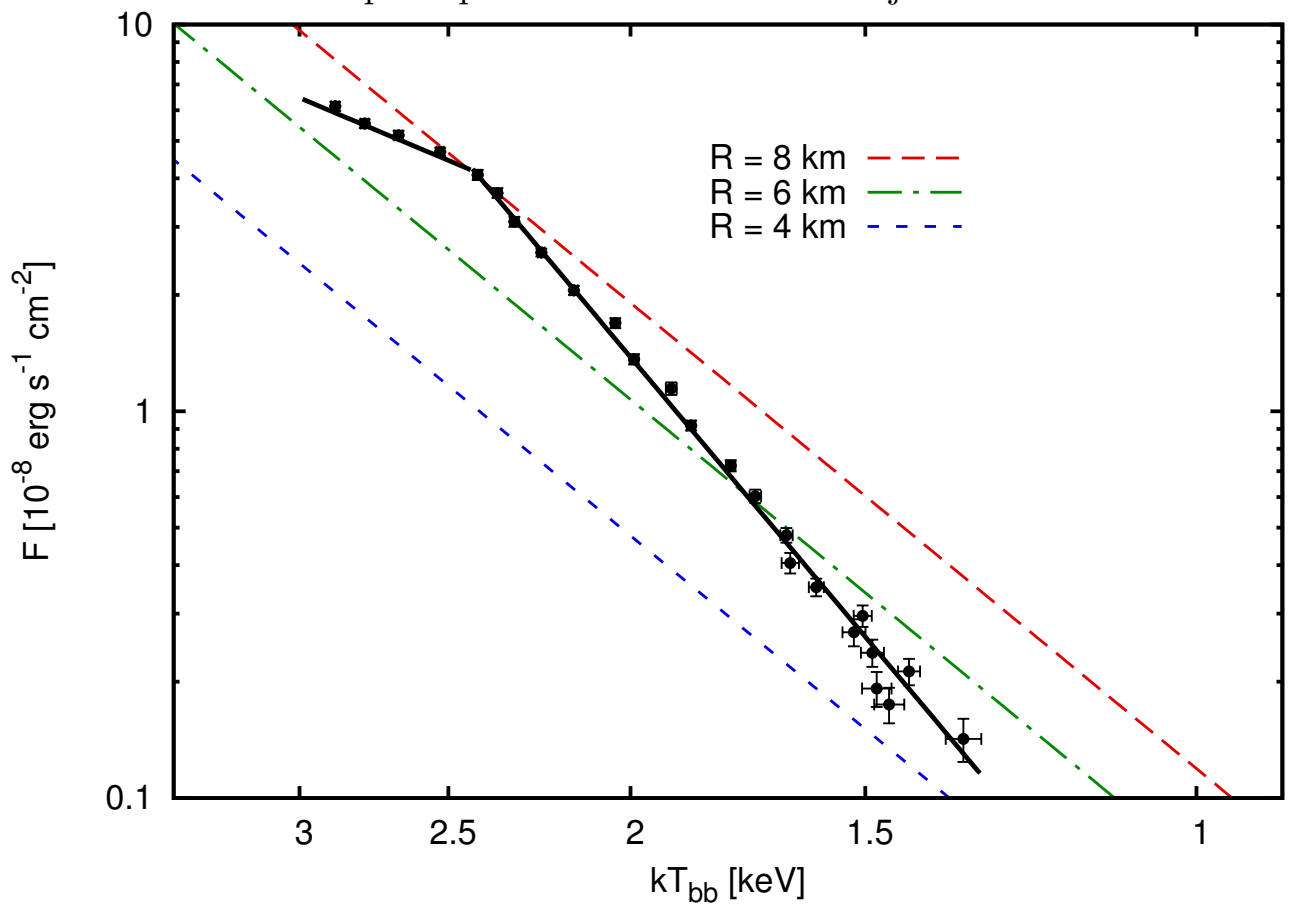

Figura 5.5: Los mismos datos que en la Figura 5.4, combinados a 25 puntos. Los datos son ajustados por una ley de potencias quebrada con índices $v_{1}=2.0 \pm 0.3$ y $v_{2}=5.72 \pm 0.06$ arriba y debajo del quiebre, respectivamente. El quiebre está a $k T_{\mathrm{bb}}=2.43 \pm 0.02 \mathrm{keV}$. 


\subsection{Discusión}

Presentamos aquí el primer análisis sistemático de la fase de enfriamiento de todos los estallidos termonucleares de rayos X detectados con $R X T E$ en el LMXB ultra-compacta 4U 1820-30. La muestra contiene 16 erupciones tipo-I con expansión fotosférica (PRE) con duraciones de entre 20 y $30 \mathrm{~s}$, todos ellos detectados cuando la fuente estaba en el estado duro o en la transición del estado duro al estado blando a relativamente baja luminosidad. Las temperaturas en el punto de contacto, así como el flujo bolométrico, las fluencias y escala de tiempos de la cola de enfriamiento exponencial de todas las erupciones son consistentes entre sí, dando apoyo a la idea que las erupciones de 4U 1820-30 son muy similares entre sí. Además, contrariamente a lo que se espera de un cuerpo negro de radio constante, la fase de enfriamiento no sigue la relación $F \propto\left(k T_{\mathrm{bb}}\right)^{4}$. De hecho, la relación entre el flujo bolométrico y la temperatura del cuerpo negro ha resultado mejor ajustada por una ley de potencias quebrada de índices $2.0 \pm 0.3$ y $5.72 \pm 0.06$. La desviación observada de la relación $F \propto\left(k T_{\mathrm{bb}}\right)^{4}$ no permite usar la fase de enfriamiento de las erupciones de rayos X para inferir la masa y el radio de la NS en 4U 1820-30 con gran precisión como fuera señalado por Güver et al. (2010).

La tendencia que encontramos en la fase de enfriamiento de las erupciones de rayos $\mathrm{X}$ en 4U 1820-30 es similar a la de las erupciones duras no-PRE de 4U 1636-53 (Zhang et al., 2011). En ambos casos, el flujo bolométrico decae más rápido que lo esperado para un cuerpo negro de radio constante. Esto se refleja en el valor del índice de ley de potencia $\left(v_{2}=5.72 \pm 0.06\right)$ que mejor ajusta la fase de enfriamiento de los estallidos por debajo de $k T_{\mathrm{bb}} \sim 2.43 \mathrm{keV}$ (ver Figura 5.5) y también en el valor que mejor se ajusta para una sola ley de potencia $(v=4.95 \pm 0.03)$ para toda la fase de enfriamiento. Teniendo en cuenta el conjunto total de estallidos en 4U 1820-30 observados con $R X T E$, no hay región del diagrama $F$ vs. $k T_{\text {bb }}$ que pueda ajustarse por una ley de potencia con índice igual a 4. Nuestros resultados están en contradicción con los de Güver et al. (2010), quienes analizaron los primeros cinco estallidos de 4U 1820-30 observados con $R X T E$ (indicados con una símbolo $\dagger$ en nuestra Tabla 5.1). Güver et al. (2010) mostraron que durante la fase de enfriamiento, tres de estas explosiones seguían la relación $F \propto\left(k T_{\mathrm{bb}}\right)^{4}$, mientras que las otras dos erupciones fueron descartadas por ellos, ya que, según su explicación, estas erupciones mostraron fluctuaciones significativas y continuas en el radio aparente. Las tres erupciones analizadas por Güver et al. (2010) se incluyen en nuestra Figura 5.4, donde resulta evidente que ninguna de las erupciones sigue la relación $F \propto\left(k T_{\mathrm{bb}}\right)^{4}$, incluso en el rango estrecho de flujo (1.0-6.0 $\times 10^{-8} \mathrm{erg} \mathrm{cm}^{-2} \mathrm{~s}^{-1}$ ) mostrado en su Figura 3 (Güver et al., 2010). Ajustando una ley de potencia a estos tres estallidos en ese rango de flujo, obtenemos un índice de $4.8 \pm 0.16$ (error a 1- $\sigma$ con $\chi^{2}=15$ para 16 grados de libertad), el cual resulta significativamente diferente de 4 . Ajustando una ley de potencias a toda la muestra de explosiones en el mismo rango de flujo obtenemos un índice de $4.68 \pm 0.08$ (error a $1-\sigma$ con $\chi^{2}=162$ para 98 grados de libertad). Observamos, además que, a partir de nuestro análisis, todas las erupciones de 4U 1820-30 observadas con RXTE son consistentes y siguen la misma tendencia en el diagrama de flujo-temperatura durante la fase de enfriamiento, incluyendo las tres explosiones estudiadas por Güver et al., y también las dos explosiones que ellos descartan. 
El comportamiento de la relación $F$ vs. $k T_{\mathrm{bb}}^{4}$ durante la fase de enfriamiento de las erupciones de rayos X en $4 \mathrm{U}$ 1820-30 podría deberse a tres razones diferentes - no mutuamente excluyentes-: (i) variaciones en el área de emisión de la estrella durante esta fase; (ii) cambios de el factor de corrección de color, $f_{\mathrm{c}}=T_{\mathrm{bb}} / T_{\mathrm{eff}}$, que representa el endurecimiento del espectro debido a la dispersión (Compton) de electrones en la atmósfera de la estrella (London et al., 1986) durante la fase de enfriamiento; (iii) la presencia de una fuente de calor que incrementa la temperatura observada, por ejemplo, producto de la quema nuclear de $\mathrm{H}$ residual durante la fase de enfriamiento de las erupciones. Además, otra posibilidad sería que los espectros subyacentes no sean en realidad cuerpos negros.

Si el factor $f_{\mathrm{c}}$ permanece constante durante la fase de enfriamiento, las variaciones en $R_{\mathrm{bb}}$ pueden explicarse por cambios en el área de emisión de la estrella. En ese caso, si la región caliente se forma lejos del polo de rotación, debería ser posible observar oscilaciones durante los estallidos (ver, por ejemplo, Strohmayer \& Bildsten, 2006). Sin embargo, ninguna oscilación fue detectada en los estallidos de 4U 1820-30 (Galloway et al., 2008; Watts, 2012). Alternativamente, si el área de emisión no cambia, las variaciones en $f_{\mathrm{c}}$ podrían explicar estas diferencias. En términos de los parámetros espectrales, el factor de corrección de color se puede expresar como:

$$
f_{\mathrm{c}}=\frac{T_{\mathrm{bb}}}{T_{\mathrm{eff}}}=\sqrt{\frac{R_{\infty}}{R_{\mathrm{bb}}}}=\sqrt{\frac{R_{\infty}}{d \sqrt{\frac{F}{\sigma T_{\mathrm{bb}}^{4}}}}}=\sqrt{\frac{R(1+z)}{d \sqrt{\frac{F k^{4}}{\sigma}}}} k T_{\mathrm{bb}},
$$

donde $R_{\infty}$ es el radio de la NS visto por un observador distante, $z$ es el corrimiento al rojo gravitacional en la superficie de la estrella, $d$ es la distancia a la fuente, $R$ su radio, y $\sigma$ la constante de Stefan-Boltzmann.

En la Figura 5.6 mostramos el factor de color deducido en función de la luminosidad inferida en unidades de Eddington. El factor de corrección de color se deduce a partir de los parámetros espectrales suponiendo $R=14 \mathrm{~km}, z=0.19$ y $d=7.6 \mathrm{kpc}$. Para estimar la luminosidad en unidades de Eddington suponemos que el flujo alcanza el límite de Eddington en el punto de contacto en cada estallido, y que la emisión es isotrópica. Promediando el flujo en el pico de las 16 explosiones obtenemos $F_{\text {Edd }}=6 \times 10^{-8} \mathrm{erg} \mathrm{cm}^{-2} \mathrm{~s}^{-1}$. En el gráfico mostramos también los modelos teóricos de $f_{\mathrm{c}}$ de Suleimanov et al. (2011) que corresponden a atmósferas con tres composiciones químicas diferentes: $\mathrm{H}$ puro, abundancia solar y He puro, respectivamente. A partir de luminosidades cercanas a la de Eddington, ya que el flujo disminuye, el valor de $f_{\mathrm{c}}$ obtenido disminuye ligeramente hasta alcanzar un valor mínimo de $f_{\mathrm{c}} \sim 1.4$ cercano a $L / L_{\text {Edd }}=0.6$. Después de esto, el factor de corrección de color inferido comienza a aumentar de nuevo, yendo más rápido para $L / L_{\mathrm{Edd}}<0.2$ en la cola de enfriamiento de los estallidos, cerca al nivel de emisión persistente, donde los errores en el ajuste espectral se vuelven importantes y los modelos son menos precisos.

A pesar de que los modelos no se ajustan bien a los datos, lo que nos impide restringir la composición de la atmósfera, nuestros resultados son incompatibles con una atmósfera de tipo solar debido a que los datos no muestran ninguna caída en $L / L_{\mathrm{Edd}}<0.2$. Al comienzo del enfriamiento, los datos son consistentes con modelos enriquecidos con He, que concuerdan con la composición química del material acretado (Rappaport et al., 1987), deducidos a partir de las 


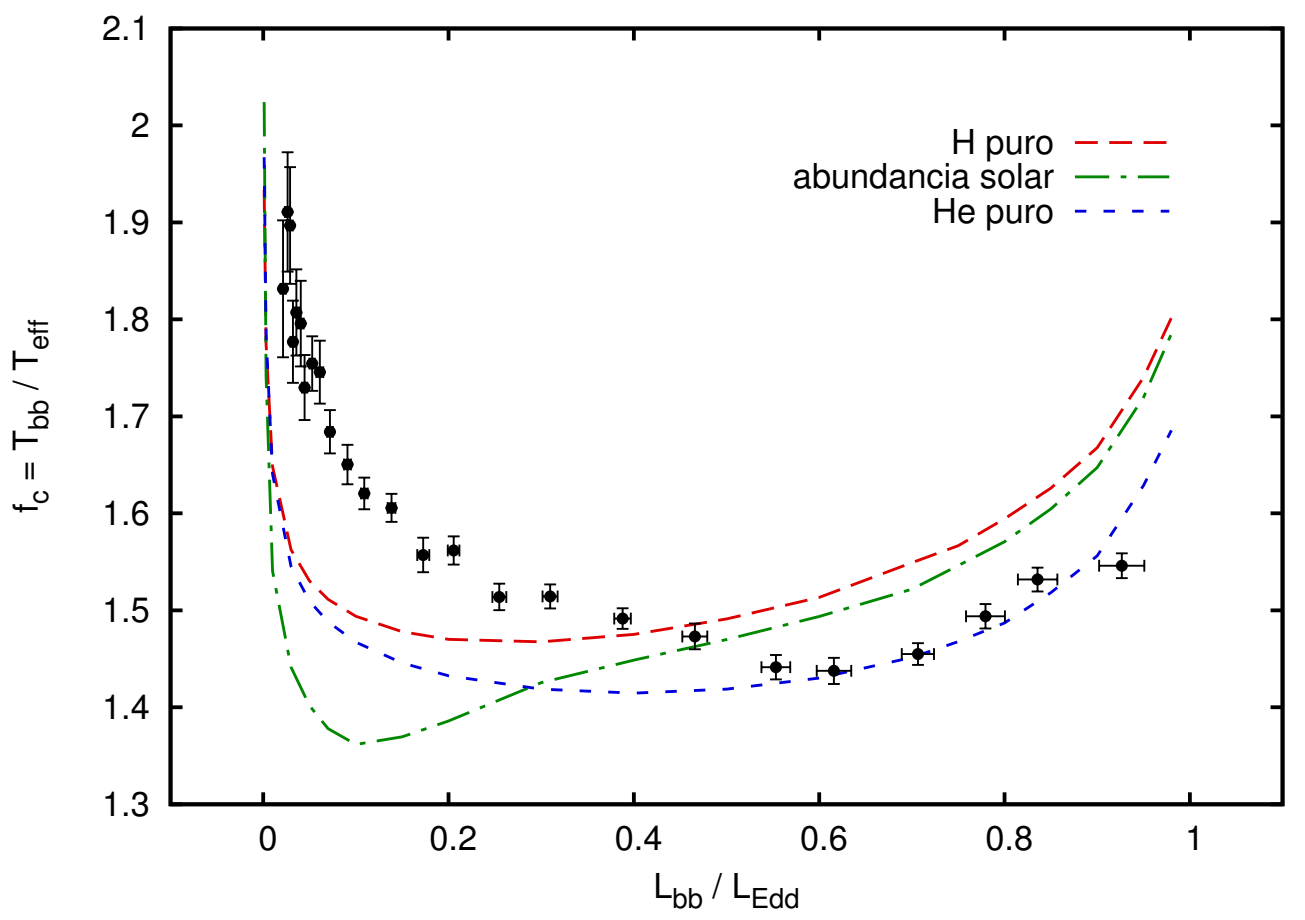

Figura 5.6: Factor de corrección de color promedio, $f_{\mathrm{c}}$, como función de unidades de la luminosidad de Eddington para los 16 estallidos de rayos X en 4U 1820-30. Los puntos corresponden a toda la muestra de estallidos en 25 bins, usando los datos de la Figura 5.5 y la Ecuación 5.1. Líneas punteadas corresponden a modelos de $f_{\mathrm{c}}$ para tres diferentes composiciones químicas de una atmósfera de NS (Suleimanov et al., 2011), indicadas en la legenda.

propiedades de la enana blanca compañera. Por otra parte, la forma de $f_{\mathrm{c}}$ en el gráfico es similar a la de la explosión no-PRE en 4U 1636-53 (Zhang et al., 2011).

Observamos también que la distribución de $k T_{\mathrm{bb}}$ a niveles de flujos bolométricos se ensancha cerca a la cola del estallido (Figura 5.4). Esto podría deberse a diferencias en la emisión subyacente procedente de la superficie de la NS, lo que podría estar indicando que la acreción persistente juega un rol importante cuando la emisión de la erupción cesa, o bien que una pequeña fracción residual de $\mathrm{H}$, no se ha consumido completamente antes de alcanzar el punto de contacto, durante la cola de la erupción. La combustión del H es más lenta que la combustión del He debido a que el decaimiento $\beta$ dentro del cadena nuclear es conducido por la fuerza débil dando lugar a una escala de tiempo más larga que la correspondiente al proceso triple- $\alpha$. La posibilidad de que esta fuente de calor opere durante la fase de enfriamiento de las erupciones de rayos X (Cumming, 2003), podría explicar el índice de ley de potencia más grande que cuatro obtenido en la relación entre el flujo y la temperatura del cuerpo negro. 
112 CAPÍTULO 5. ENFRIAMIENTO DE LA ESTRELLA DE NEUTRONES EN 4U 1820-30 


\section{Parte IV}

\section{Aportes al estudio de estrellas de neutrones en teorías alternativas a la gravedad}





\section{Breve descripción de la cuarta parte}

En esta parte de la Tesis se presentan los aportes realizados al estudio de NSs en teorías alternativas a la GR.

En el Capítulo 6 presentaremos los resultados obtenidos bajo la teoría $f(R)=R+\alpha R^{2}$ en el formalismo métrico. Mostraremos que, contrariamente a lo que ocurre en GR, las configuraciones halladas presentan características muy particulares en los perfiles de masa encerrada. En regiones donde la derivada segunda de la EoS utilizada representa transiciones de fase, por ejemplo, entre la corteza y el núcleo, la masa encerrada de las configuraciones estáticas calculadas decrece con la coordenada radial, de manera anti-intuitiva. Estos resultados fueron publicados en un artículo de la revista General Relativity and Gravitation (Orellana et al., 2013).

En el Capítulo 7 mostramos los resultados correspondientes a un estudio similar realizado bajo el formalismo de Palatini, donde la métrica depende no sólo de las coordenadas sino también de la conexión afín, de manera independiente, y para el cual encontramos resultados muy similares a los del formalismo métrico. Cabe aclarar que si bien en GR las formulaciones métrica y de Palatini son equivalentes, esto no ocurre para otras teorías como la gravedad cuadrática $f(R)$ de interés aquí. Los resultados aquí expuestos serán enviados próximamente para ser publicados en un artículo de la misma revista (Teppa Pannia et al., 2016). 


\section{Capítulo 6}

\section{Estrellas de neutrones en teorías $f(R)$ cuadráticas: formalismo métrico}

\subsection{Resumen}

Los efectos causados sobre la estructura de objetos compactos por la modificación de GR bajo la generalización de la densidad lagrangiana a la forma $f(R)=R+\alpha R^{2}$, donde $R$ es el escalar de curvatura de Ricci, han comenzado a ser explorados recientemente en la literatura. Los primeros estudios mostraron que bajo esta teoría de gravedad cuadrática podrían obtenerse NSs más masivas que en GR. Por otro lado, como las diferencias entre las diferentes teorías de gravedad alternativas se ven amplificadas en el régimen de campo fuerte, es posible que las NSs puedan resultar útiles para poner cotas a los parámetros libres que estas teorías incorporan.

En este régimen, debido a la complejidad de las ecuaciones de campo, los métodos perturbativos resultan imprescindibles para tratar el problema teórico. Así, basándonos en trabajos previos en el campo, en este capítulo presentamos los resultados numéricos de la integración de las ecuaciones de estructura que describen a las NSs en gravedad $f(R)$ usando el formalismo métrico. Para poner a prueba nuestro modelo, primero recuperamos resultados correspondientes a diagramas masa-radio obtenidos por otros autores, para luego enfocarnos en rasgos característicos que surgen en los perfiles de las soluciones que describen el interior de estas estrellas. Mostramos que estos perfiles correlacionan con la forma de la derivada segunda de las aproximaciones analíticas a las EoSs utilizadas, dando como resultado regiones donde la masa encerrada decrece con la coordenada radial, en forma anti-intuitiva.

Finalmente, reproducimos estos cálculos con una ecuación politrópica simple para separar efectos de orden cero propios de la teoría de gravedad modificada utilizada y comparamos los resultados con los obtenidos en el marco de la GR. 


\subsection{Introducción}

La interpretación de las observaciones cosmológicas actuales en el modelo cosmológico estándar requieren la presencia de un contenido de materia exótica para dar cuenta de la expansión acelerada del universo (Amanullah et al., 2010; Komatsu et al., 2011; Larson et al., 2011; Percival et al., 2010; Riess et al., 2009). A lo largo de la última década, se han ido desarrollando una serie de modelos cosmológicos alternativos con el fin de poder interpretar estos datos sin la necesidad de involucrar una componente desconocida al tensor de energía y materia (ver, por ejemplo, Bolejko et al., 2011). En este contexto una de las alternativas que surgieron la constituyen las teorías extendidas a la gravitación (Capozziello \& Faraoni, 2011; de Felice \& Tsujikawa, 2010; Sotiriou \& Faraoni, 2010), basadas en correcciones y generalizaciones de la GR.

Como se explicara ya en el Capítulo 1, elegimos enfocarnos en una familia de estas teorías, denominadas teorías de gravedad $f(R)$, obtenidas a partir de la modificación de la acción de Einstein-Hilbert. Para ello, la densidad lagrangiana usual es generalizada reemplazando el escalar de curvatura de Ricci, $R$, por una función de él, o por invariantes del tensor de curvatura de mayor orden, como $R^{2}$ (Capozziello \& Faraoni, 2011).

Estos modelos, a su vez, se construyen de manera que satisfagan las cotas impuestas por los estudios detallados llevados a cabo en laboratorios terrestres o en el propio Sistema Solar (Hu \& Sawicki, 2007; Jaime et al., 2011; Miranda et al., 2009; Starobinsky, 2007). En particular, la alternativa más simple, denominada gravedad cuadrática, donde $f(R)=R+\alpha R^{2}$, ha sido largamente estudiada como una base para un modelo cosmológico viable, que puede explicar la expansión acelerada del Universo siendo además consistente con las anisotropías observadas en el fondo cosmológico de radiación (de Felice \& Tsujikawa, 2010). Pero, contrariamente al caso de la gravedad en el régimen de campo débil, que ha sido sujeta a numerosos experimentos, el régimen de campo gravitacional fuerte, relevante en este trabajo, dista mucho de estar restringido por las observaciones (ver, por ejemplo, DeDeo \& Psaltis, 2003).

Usando un modelo más detallado de la estructura de una estrella compacta, otros autores obtuvieron un conjunto de ecuaciones de TOV modificadas que describen una distribución de masa esféricamente simétrica y en equilibrio hidrostático, en gravedad $f(R)$, derivando soluciones que reproducen su comportamiento en el límite de campo gravitacional débil (ver, por ejemplo, Babichev \& Langlois, 2010, en el contexto de teorías de gravedad escalares y tensoriales). Además, trabajando con una aproximación perturbativa para resolver las ecuaciones de campo, Cooney et al. (2010) y Santos (2012) encontraron relaciones masa-radio para estrellas compactas utilizando una EoS politrópica. Más recientemente, Arapoğlu et al. (2011) y Deliduman et al. (2012) aplicaron el mismo método utilizando EoSs realistas para NSs en teorías de gravedad cuadrática en $R$ y $R_{\mu \nu} R^{\mu v}$, respectivamente.

Las relaciones masa-radio obtenidas por Arapoğlu et al. (2011) y Deliduman et al. (2012) indican que bajo estas teorías de gravedad $f(R)$, sería posible construir estrellas con masas aún mayores a las NS más masivas que se conocen, tales como la NS en PSR J1614-2230, con $M=$ $1.97 \pm 0.04 \mathrm{M}_{\odot}$ (Demorest et al., 2010). La gravedad cuadrática introduce un nuevo parámetro al modelo a través del coeficiente $\alpha$, asociado al término $R^{2}$. La libertad en la elección de este 
parámetro permite que algunas EoSs, excluidas en la GR por no ser capaces de dar cuenta de estrellas tan masivas, puedan reconciliarse con estas observaciones. Motivados por estos resultados, investigamos en detalle la estructura de NSs en este modelo considerando dos EoS diferentes. Una de ellas es una aproximación politrópica que usamos para separar los efectos de orden cero introducidos por la teoría de gravedad modificada utilizada, respecto a la GR, mientras que la segunda provee una representación realista de la materia nuclear a alta densidad.

\subsection{Ecuaciones de la estructura estelar}

Como se detalló en el Capítulo 1, las ecuaciones que definen la estructura de una NS en GR se deducen a partir de proponer un elemento de línea, ds, estático y con simetría esférica para la métrica:

$$
\mathrm{d} s^{2}=-e^{2 \Phi(r)} c^{2} \mathrm{~d} t^{2}+e^{2 \Lambda(r)} \mathrm{d} r^{2}+r^{2}\left(\mathrm{~d} \theta^{2}+\sin ^{2} \theta \mathrm{d} \varphi^{2}\right)
$$

Luego, considerando las ecuaciones de Einstein para un tensor ideal de energía y momento $T_{v}^{\mu}=$ $\operatorname{diag}\left\{-\rho c^{2}, p, p, p\right\}$, las ecuaciones TOV que describen la estructura de las estrellas de neutrones en GR, pueden ser expresadas como:

$$
\begin{aligned}
\frac{\mathrm{d} m(r)}{\mathrm{d} r} & =4 \pi r^{2} \rho(r) \\
\frac{\mathrm{d} p(r)}{\mathrm{d} r} & =\frac{c^{2} \rho(r)+p(r)}{2 G m(r)-c^{2} r} G\left(\frac{4 \pi}{c^{2}} r^{2} p(r)+\frac{m(r)}{r}\right) \\
e^{-2 \Lambda(r)} & =1-\frac{2 G m(r)}{c^{2} r} \\
\frac{\mathrm{d} \Phi(r)}{\mathrm{d} r} & =-\frac{1}{\left(c^{2} \rho(r)+p(r)\right)} \frac{\mathrm{d} p}{\mathrm{~d} r}
\end{aligned}
$$

donde $m(r)$ es la masa relativista total encerrada en una esfera de radio $r$. Las funciones $\rho(r)$ y $p(r)$ son, respectivamente, la densidad de masa (energía) y la presión a dicho radio. Explicitando entonces una relación entre $\rho$ y $p$, la llamada EoS, las ecuaciones TOV pueden ser resueltas desde el interior, suponiendo un cierto valor para la densidad central, $\rho(r=0)=\rho_{c}$, e integrando el sistema hasta que la presión se anula en la superficie, $p(r=R)=0$. De esta manera quedan definidos el radio de la estrella, $R$, y su masa, $M=m(R)$. Así, cada valor de $\rho_{c}$ genera una configuración caracterizada por el par $(M, R)$. Luego, variando este parámetro, se obtiene una relación masa-radio $(M-R)$ para cada EoS.

Las ecuaciones TOV modificadas pueden ser obtenidas de las ecuaciones de campo. Agregando un nuevo término a la acción de Einstein-Hilbert:

$$
S=\frac{c^{4}}{16 \pi G} \int \mathrm{d}^{4} x \sqrt{-g}\left(R+\alpha R^{2}\right)+S_{\mathrm{m}}
$$

donde $g$ es el determinante de la métrica, $g_{\mu \nu}$, y $S_{\mathrm{m}}$ es la acción de la materia. 
Trabajando en el formalismo métrico, la variación de la acción respecto de la métrica se reduce a un sistema de ecuaciones diferenciales de cuarto grado en $g_{\mu \nu}$, lo que pone un gran obstáculo a la resolución completa del problema. Por esta razón, decidimos adoptar un esquema perturbativo presentado inicialmente por Cooney et al. (2010) y Arapoğlu et al. (2011). Reescribiendo $f(R)=R+\alpha R^{2}=R(1+\beta)$, consideramos la función $f(R)$ como una perturbación sobre un fondo de GR. Luego, la cantidad adimensional $\beta \equiv \alpha R$ comprenderá la desviación de la GR de manera que el método perturbativo resultará aplicable sólo si $|\beta| \ll 1$. Bajo esta condición, podemos trabajar entonces con ecuaciones de movimiento hasta el primer orden en $\beta \sin$ imponer ninguna restricción a nivel de la acción y asegurando que se cumpla la naturaleza del principio variacional (Cooney et al., 2009). Ignorando términos de $\boldsymbol{O}\left(\beta^{2}\right)$ o superiores, para esta elección particular de $f(R)$, las ecuaciones de campo se reducen a

$$
G_{\mu \nu}+\alpha\left[2 R\left(R_{\mu \nu}-\frac{1}{4} R g_{\mu \nu}\right)+2\left(g_{\mu \nu} \square R-\nabla_{\mu} \nabla_{v} R\right)\right]=\frac{8 \pi G}{c^{4}} T_{\mu \nu}
$$

donde $G_{\mu \nu}=R_{\mu \nu}-\frac{1}{2} R g_{\mu \nu}$ es el tensor de Einstein. En el límite $\alpha \longrightarrow 0, \beta \longrightarrow 0$, se recuperan las ecuaciones de campo de GR (ver, por ejemplo, Capozziello \& Faraoni, 2011).

Suponiendo que el elemento de línea estático y con simetría esférica, viene dado por la Ecuación 6.1, bajo el tratamiento perturbativo es posible expandir las funciones dentro de la métrica en un término dominante (no perturbado), que notamos con el índice 0 , y un término perturbativo, que notamos con subíndice 1. De esta manera, a primer orden en $\beta, \Lambda=\Lambda_{0}+\beta \Lambda_{1} \mathrm{y}$ $\Phi=\Phi_{0}+\beta \Phi_{1}$, mientras que las cantidades hidrodinámicas quedan definidas perturbativamente como: $\rho=\rho_{0}+\beta \rho_{1}$ y $p=p_{0}+\beta p_{1}$ (Arapoğlu et al., 2011). Así surgen entonces nuevas restricciones al parámetro $\beta$ por las cotas impuestas por $\beta \Phi_{1} \ll \Phi_{0}, \beta \Lambda_{1} \ll \Lambda_{0}, \beta \rho_{1} \ll \rho_{0}$, y $\beta p_{1} \ll p_{0}$. A partir de aquí, utilizaremos variables primadas para indicar derivadas en la coordenada radial.

Siguiendo a Cooney et al. (2009) y Arapoğlu et al. (2011), definimos la masa suponiendo que la solución para la métrica exterior tiene la misma forma que la solución de Schwarzschild en GR, es decir,

Para la solución interior,

$$
e^{-2 \Lambda(r)}=1-\frac{2 G M_{*}}{c^{2} r}
$$

$$
e^{-2 \Lambda(r)}=1-\frac{2 G m(r)}{c^{2} r}
$$

donde $m$ también admite una expansión perturbativa $m=m_{0}+\beta m_{1}$, siendo $m_{0}$ la masa a orden cero que se obtiene integrando el sistema de ecuaciones TOV de GR (6.2).

Con estas consideraciones, y teniendo en cuenta que $\rho_{0}$ y $p_{0}$ satisfacen las ecuaciones de Einstein, las ecuaciones TOV modificadas resultan:

$$
\begin{aligned}
& \frac{\mathrm{d} m}{\mathrm{~d} r}=4 \pi r^{2} \rho-2 \beta[\overbrace{4 \pi r^{2} \rho_{0}-\frac{c^{2}}{8 G} r^{2} R_{0}}^{A / 2}+\overbrace{\left(2 \pi \rho_{0} r^{3}-\frac{c^{2}}{G} r+\frac{3}{2} m_{0}\right) \frac{R_{0}^{\prime}}{R_{0}}}^{B / 2} \overbrace{r\left(\frac{c^{2}}{2 G} r-m_{0}\right) \frac{R_{0}^{\prime \prime}}{R_{0}}}^{-C / 2}] \\
& \frac{c^{2} p+p}{\mathrm{~d} r}=\frac{c^{2} \rho+p}{2 G m-c^{2} r} G\left\{\left(\frac{4 \pi}{c^{2}} r^{2} p+\frac{m}{r}\right)-2 \beta\left[\frac{4 \pi}{c^{2}} r^{2} p_{0}+\frac{c^{2}}{8 G} r^{2} R_{0}+\left(\frac{2 \pi}{c^{2}} p_{0} r^{3}+\frac{c^{2}}{G} r-\frac{3}{2} m_{0}\right) \frac{R_{0}^{\prime}}{R_{0}}\right]\right\}
\end{aligned}
$$


Notar que $2 \beta[\ldots]$ son las correcciones a primer orden en $\beta$ de los gradientes $\mathrm{d} m / \mathrm{d} r$ y $\mathrm{d} p / \mathrm{d} r$, respectivamente, que deben ser evaluados a orden cero, es decir, usando las cantidades que surgen integrando el sistema TOV. Remarcamos acá que $\mathrm{d} p / \mathrm{d} r$ no depende de $R_{0}^{\prime \prime}$. Los términos correspondientes a las derivadas de la masa son indicados, según: $\mathrm{d} m / \mathrm{d} r=4 \pi r^{2} \rho-\beta[A+B+C]$, ya que estudiaremos la contribución de cada uno de estos términos más adelante.

La obtención de las ecuaciones exactas 6.10 y 6.11 es imposible ya que $\beta$ involucra otras derivadas a través del escalar de curvatura, $R$, que vuelven a $\beta$ dependiente de $r$ de una manera compleja ${ }^{1}$. Por lo tanto, integramos las ecuaciones 6.10 y 6.11 suponiendo que $\beta$ es bien aproximado por $\beta \simeq \hat{\beta} \equiv \alpha R_{0}$, donde $R_{0}$ es el escalar de Ricci calculado localmente en GR y $\alpha$ es un parámetro constante con unidades de distancia al cuadrado, compatible con lo realizado por otros autores (por ejemplo, Arapoğlu et al., 2011). Notar que

$$
R_{0}=\frac{8 \pi G}{c^{4}}\left(\rho_{0} c^{2}-3 p_{0}\right)
$$

y entonces, contrario a lo que ocurre en GR, las derivadas de la EoS, $\mathrm{d} p / \mathrm{d} \rho$ y d $\mathrm{d}^{2} p / \mathrm{d} \rho^{2}$, también entran en el cálculo del sistema de ecuaciones formado por 6.10 y 6.11, a través de $R_{0}^{\prime}$ y $R_{0}^{\prime \prime}$.

En gravedad $f(R)$ el peso de la perturbación queda regulado por el valor del parámetro $\alpha$. En nuestro trabajo, nos restringimos a las cotas impuestas por Santos (2010), y las referencias allí citadas, que apuntan a valores de $\alpha$ en el rango $10^{8} \mathrm{~cm}^{2}<\alpha / 3<10^{10} \mathrm{~cm}^{2}$, basados en observaciones astronómicas en el Sistema Solar y experimentos nucleares en laboratorios terrestres.

\subsection{Ecuaciones de estado}

Para completar el sistema de ecuaciones formado por 6.10 y 6.11 es necesario dar una relación explícita entre $p$ y $\rho$, llamada EoS. La EoS contiene información de las condiciones microfísicas de la materia en todo el interior de las NSs, cubriendo una gran cantidad de décadas en densidad. Como las propiedades de la materia en las regiones más internas de las NSs son esencialmente desconocidas o muy poco conocidas, en general se proponen diferentes EoS alternativas con las que se construyen los modelos de NSs para luego contrastarlas con las masas y radios de algunas de estas estrellas obtenidas a través de las observaciones.

Para poder resolver las ecuaciones de estructura de cuarto orden de NSs en teorías de gravedad modificadas, tales como la gravedad cuadrática, se necesitan representaciones analíticas de las EoS, ya que, en estos casos, las técnicas usuales de interpolación fallan a la hora de representar con precisión las derivadas de orden superior (Gungor \& Yavuz Eksi, 2011). Haensel \& Potekhin (2004) desarrollaron un procedimiento termodinámicamente consistente para obtener representaciones analíticas de dos EoSs. Los autores calcularon los coeficientes del ajuste de una expansión

${ }^{1}$ El escalar de Ricci en término de las funciones que involucran a la métrica (Ecuación 6.1) se expresa como:

$$
R=\frac{2 e^{-2 \Lambda}\left[r^{2}\left\{\Phi^{\prime} \Lambda^{\prime}+\left(\Phi^{\prime}\right)^{2}+\Phi^{\prime \prime}\right\}+2 r\left(\Phi^{\prime}-\Lambda^{\prime}\right)-e^{2 \Lambda}+1\right]}{r^{2}}
$$


polinómica que abarca a la EoS en los regímenes asociados tanto a la corteza como al núcleo. Sin embargo, debe enfatizarse que tales aproximaciones a las EoS fueron obtenidas de manera que reproduzcan con una cierta fidelidad a la relación de orden cero entre $\rho$ y $p$, dejando de lado sus derivadas, ya que éstas no son necesarias para calcular la estructura de NSs en GR. Es por ello que el uso de estas aproximaciones involucrando sus derivadas durante el cálculo, como en el caso que estamos interesados aquí (es decir, $\mathrm{d} p / \mathrm{d} \rho \mathrm{y} \mathrm{d}^{2} p / \mathrm{d} \rho^{2}$ ), debe realizarse con un cuidado especial.

Teniendo esto en cuenta, y con el objeto de comparar nuestros resultados con aquellos publicados previamente en la literatura, calculamos las relaciones masa-radio $(M-R)$ considerando dos EoS diferentes: SLY (Douchin \& Haensel, 2001; Haensel \& Potekhin, 2004) y POLY (Silbar \& Reddy, 2004). La primera es una EoS realista, obtenida de forma tal que pueda representar de manera adecuada el comportamiento de la materia nuclear a alta densidad. Su parametrización analítica viene dada por

$$
\begin{aligned}
\zeta= & \frac{a_{1}+a_{2} \xi+a_{3} \xi^{3}}{1+a_{4} \xi} f_{0}\left(a_{5}\left(\xi-a_{6}\right)\right) \\
& +\left(a_{7}+a_{8} \xi\right) f_{0}\left(a_{9}\left(a_{10}-\xi\right)\right) \\
& +\left(a_{11}+a_{12} \xi\right) f_{0}\left(a_{13}\left(a_{14}-\xi\right)\right) \\
& +\left(a_{15}+a_{16} \xi\right) f_{0}\left(a_{17}\left(a_{18}-\xi\right)\right)
\end{aligned}
$$

donde

$$
\xi=\log \left(\rho / \mathrm{g} \mathrm{cm}^{-3}\right), \quad \zeta=\log \left(P / \mathrm{dyn}^{-2}\right)
$$

y

$$
f_{0}(x)=\frac{1}{\mathrm{e}^{x}+1}
$$

Los coeficientes $a_{i}$ están tabulados en Haensel \& Potekhin (2004). Esta expresión es la misma que utilizaron Arapoğlu et al. (2011), y la introducimos aquí para verificar nuestros resultados. La segunda EoS es una aproximación politrópica simple, dada por la relación:

$$
\zeta=2 \xi+5.29355
$$

Más allá de que ésta última no es una EoS realista y por lo tanto esté lejos de representar en profundidad una NSs, resulta un modelo simple numéricamente que nos permite estudiar los efectos de orden cero introducidos por la teoría de gravedad modificada. El valor preciso del índice adiabático, $\Gamma=\mathrm{d} \log p / \mathrm{d} \log \rho=\mathrm{d} \zeta / \mathrm{d} \xi$, no resulta relevante mientras que $\Gamma$ permanece como una cantidad derivable. Para encontrar mayores restricciones al índice $\Gamma$, sugerimos la lectura de Read et al. (2009) que apunta a valores un poco mayores a los que adoptamos aquí.

\subsection{Método numérico}

La resolución del sistema de ecuaciones diferenciales ordinarias formado por 6.10 y 6.11 requiere su integración desde el centro hasta la superficie de la NS, usando la EoS elegida. Una vez 
que la integración alcanza la superficie, la pareja de valores $(M, R)$ queda establecida. Para realizar la integración numérica en la coordenada radial, desarrollamos un código numérico basado en el método Runge-Kutta de cuarto orden. Incorporamos una paso variable en la coordenada radial que se acorta sistemáticamente a medida que nos aproximamos a la superficie de la NS, para dar cuenta de las variaciones rápidas de todas las cantidades físicas en esa región, y de manera que, a su vez, el código resuelva la parte interna sin requerir un elevado número de pasos superfluos.

Durante el bucle de Runge-Kutta, resolvemos también los términos asociados a las componentes de la métrica: $g_{t t}$ y $g_{r r}$, que resultan esenciales para garantizar el criterio de validez del tratamiento perturbativo. En cada paso, integramos primero las ecuaciones TOV en GR para encontrar los valores correspondientes a las soluciones de orden cero que luego usamos como base para calcular las soluciones perturbativas de primer orden.

La integración numérica comienza desde el centro, fijando una densidad central arbitraria, $\rho_{c}$, y termina en la superficie que definimos arbitrariamente a la densidad $\rho=10^{6} \mathrm{gr} \mathrm{cm}^{-3}$. Esta densidad corresponde al borde externo de la corteza de la NS, que representa el límite de validez para este tipo de EoSs, concebidas asumiendo un modelo de materia nuclear a alta densidad. A partir de esa densidad, las variaciones en la masa encerrada y el radio de la estrella son mucho menores que las incertezas del problema.

\subsection{Resultados}

En la Figura 6.1 presentamos las relaciones masa-radio $(M-R)$ obtenidas para las EoS SLY y POLY (paneles izquierdo y derecho, respectivamente), usando siete valores del parámetro $\alpha$ entre -0.2 y $+0.2 \mathrm{~km}^{2}$ y considerando valores de $\rho_{c}$ en el rango de $10^{14.6} \mathrm{gr} \mathrm{cm}^{-3}$ a $10^{15.9} \mathrm{gr} \mathrm{cm}^{-3}$. Las masas máximas correspondientes a cada familia de soluciones están indicadas con cruces en cada curva. Nuestros resultados para la EoS SLY están en acuerdo con aquellos presentados previamente por Arapoğlu et al. (2011). Valores de $\alpha<0 \mathrm{~km}^{2}\left(\alpha>0 \mathrm{~km}^{2}\right)$ permiten construir estrellas con masas mayores (menores) que en GR. Además, las configuraciones correspondientes a la EoS POLY resultan mucho menos sensibles al parámetro $\alpha$ que aquellas calculadas para la EoS SLY.

En la Figura 6.2 presentamos los perfiles internos hallados para la densidad, $\rho(r)$, y la masa encerrada, $m(r)$, para valores extremos del parámetro $\alpha$ así como para el caso de GR. Los perfiles correspondientes a la densidad (y, por ende, a la presión) tienen un formato estándar, siguiendo la tendencia de los perfiles en GR, donde ambas magnitudes decrecen monótonamente con el radio. Por el contrario, en los perfiles de masa encerrada obtenidos usando la EoS realista SLY, aparecen efectos muy particulares en los casos correspondientes a $f(R)$, que se vuelven más pronunciados en las NSs más masivas consideradas $\left(\rho_{c}=10^{15.4} \mathrm{gr} \mathrm{cm}^{-3}\right)$.

Estos efectos son muy evidentes cerca de la superficie de las NS (a $r \sim 10 \mathrm{~km}$ ) donde, en una capa de espesor delgado $(\Delta r \sim 0.2 \mathrm{~km})$, se produce un decrecimiento inesperado (anti-intuitivo) en $m(r)$ que aparece antes $\left(\alpha>0 \mathrm{~km}^{2}\right)$ o después $\left(\alpha<0 \mathrm{~km}^{2}\right)$ de un pozo (pico) en los perfiles. En la Figura 6.3 mostramos el detalle de los perfiles de masa y densidad (paneles izquierdo y derecho, respectivamente) cerca de la superficie de la NS, obtenidos usando la EoS SLY para 

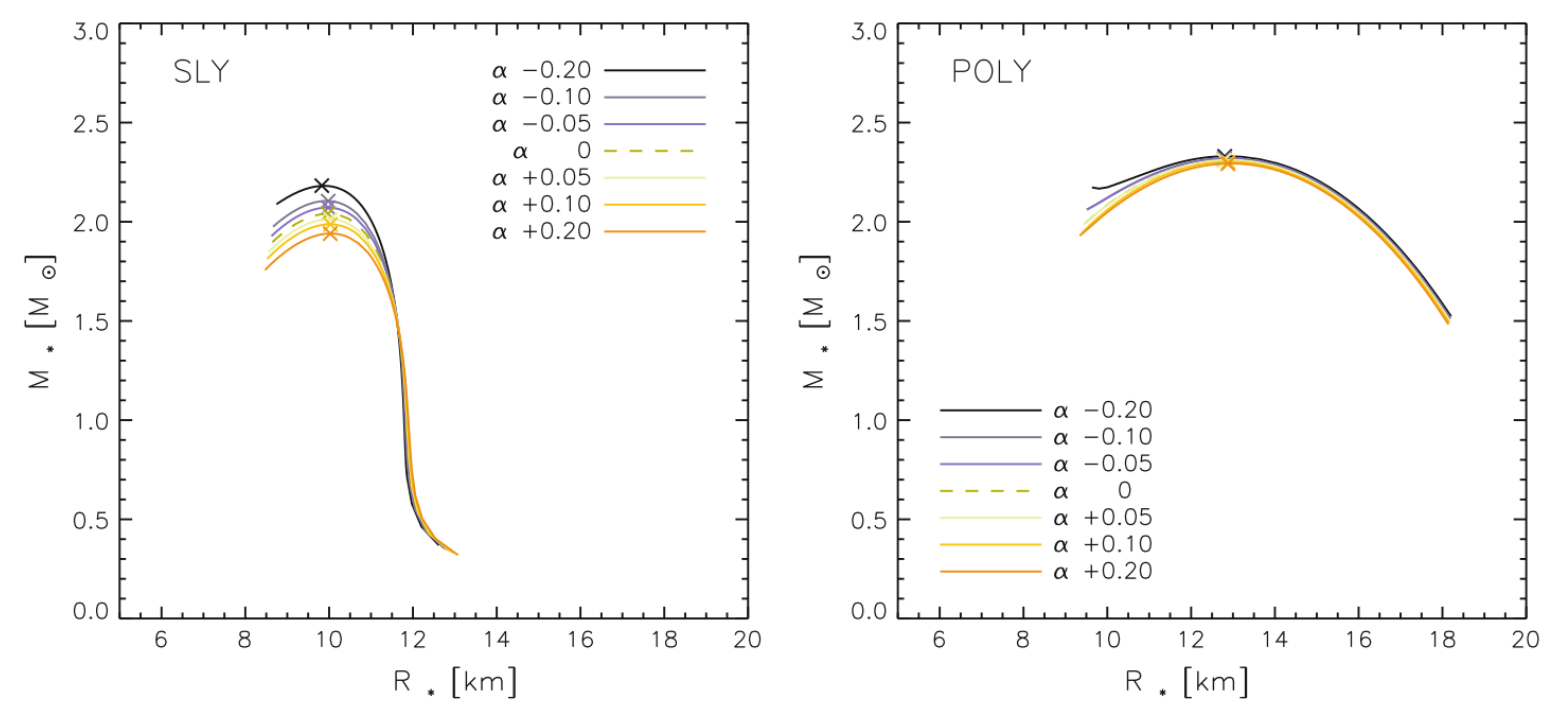

Figura 6.1: Relaciones $(M-R)$ para las EoS SLY y POLY (izquierda y derecha, respectivamente), considerando siete valores del parámetro $\alpha$, indicados en unidades de $\mathrm{km}^{2}$. Todas las curvas corresponden a soluciones obtenidas para valores de $\rho_{c}$ en el rango $10^{14.6-15.9} \mathrm{gr} \mathrm{cm}^{-3}$. Las cruces indican la masa máxima para cada caso.

$\log \rho_{c}\left[\mathrm{gr} \mathrm{cm}^{-3}\right]=15.4$ y 14.6. Más allá de que la modificación de $M_{*}$ es mayor para estrellas con densidades centrales altas, el cambio relativo en la masa total respecto del caso de GR es de aproximadamente el $10 \%$ en los dos casos estudiados.

En el marco de la GR, sólo podría esperarse un perfil de masa decreciente si se incorporara un fluido de densidad de energía negativa, ya que $\mathrm{d} m / \mathrm{d} r=4 \pi r^{2} \rho(r)$. Por el contrario, en gravedad $f(R)$ este comportamiento de los perfiles podría ser explicado como una consecuencia de la modificación de la geometría de fondo. Por el contrario, en las estrellas construidas con la EoS POLY, este comportamiento en los perfiles no se observa en ninguno de los casos analizados.

En la Figura 6.4 presentamos el perfil del cociente de los términos de la métrica $g_{r r}^{0} / g_{r r}$ a lo largo de todo el interior de la NS para la EoS SLY, considerando las mismas densidades que en la Figura 6.2. Este cociente debe ser próximo a la unidad como condición necesaria para la validez del método perturbativo utilizado. En el panel superior de esta figura graficamos también las derivadas logarítmicas de primer y segundo orden de la EoS SLY. De la comparación visual de la marcha de las curvas, se evidencia un fuerte acoplamiento entre las desviaciones perturbativas de $g_{r r}$ y la derivada segunda de la EoS.

La modificación de la componente radial de la métrica es leve, y sólo perceptible cuando la derivada de segundo orden de la EoS se torna suficientemente importante, oscilando fuertemente en el rango de densidades de $10^{11}-10^{14} \mathrm{gr} \mathrm{cm}^{-3}$. Este comportamiento no ocurre en el caso de la EoS POLY, cuya derivada logarítmica de segundo orden es nula, por lo que el cociente $g_{r r}^{0} / g_{r r} \simeq 1$ a través de todo el interior de las NSs. Es importante hacer notar que la función $\Phi(r)$ en la 

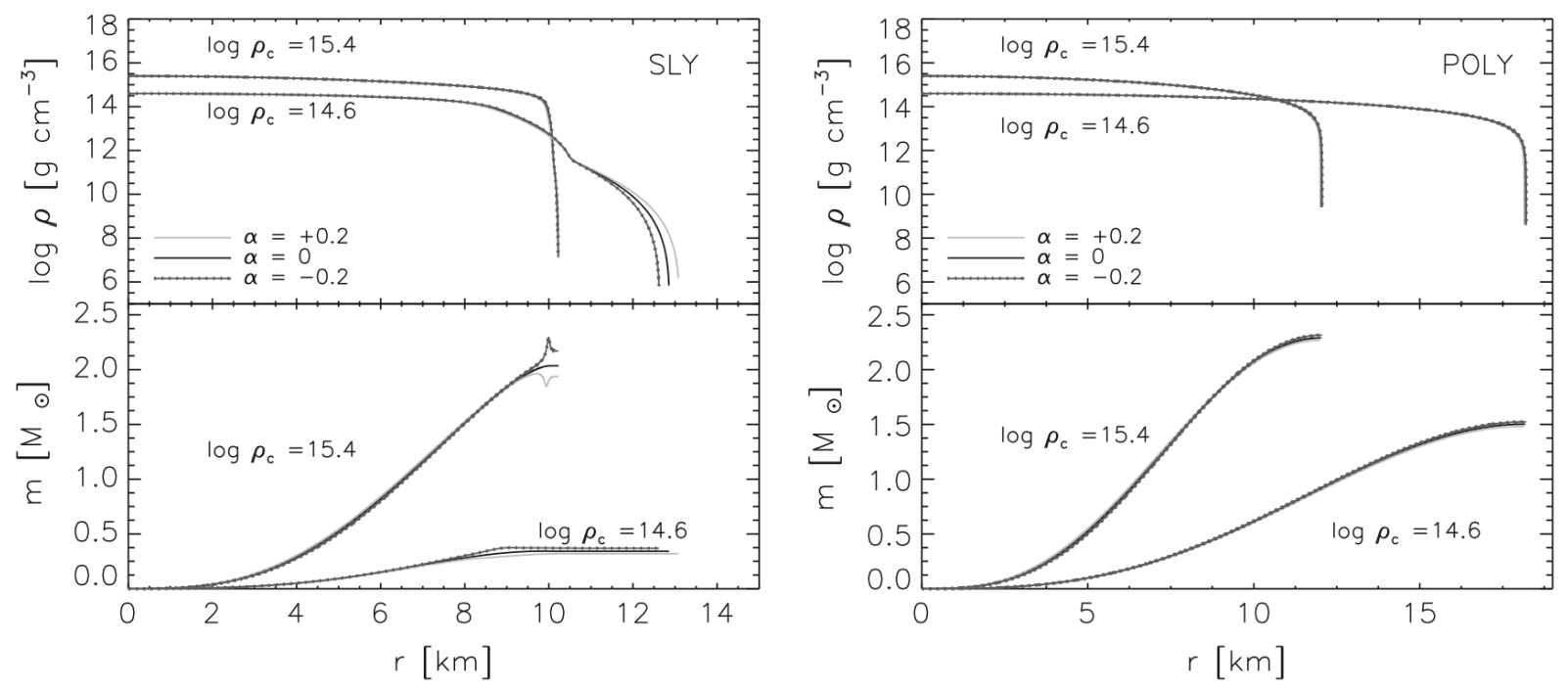

Figura 6.2: Perfiles internos de la estructura de las NSs para dos casos extremos de densidades centrales bajas y altas, $\rho_{c}=10^{14.6}$ y $10^{15.4} \mathrm{gr} \mathrm{cm}^{-3}$, para tres valores diferentes del parámetro $\alpha$ $\left(+0.2,0.0 \mathrm{y}-0.2 \mathrm{~km}^{2}\right)$, donde $\alpha=0.0$ corresponde al caso de la GR. En el panel izquierdo (derecho) se muestran los perfiles correspondientes la EoS SLY (POLY). En la Figura 6.3 presentamos el detalle del perfil de masa cerca de la superficie de la NS. Para valores bajos de la densidad central, el efecto en la masa integrada puede representar una desviación del orden de $\leq 10 \%$ del caso de GR.
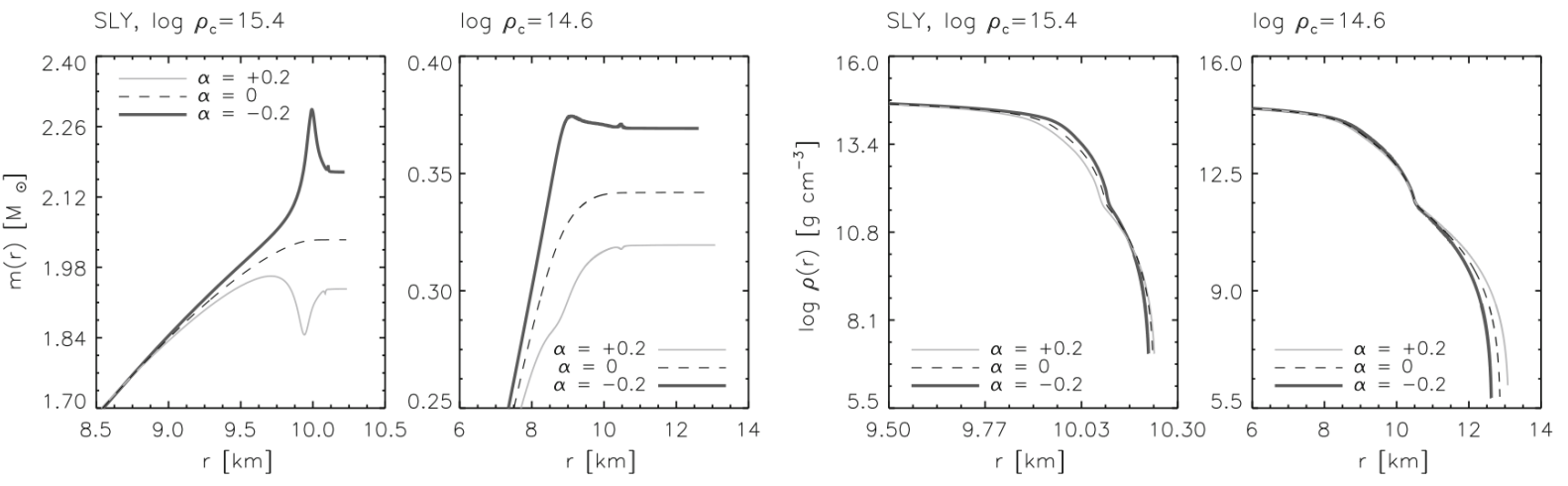

Figura 6.3: Detalle de los perfiles de densidad y masa cerca de la superficie de la NS para el caso de la EoS SLY EoS que se muestra en la Figura 6.2, para $\rho_{c}=10^{15.4}$ y $10^{14.6} \mathrm{gr} \mathrm{cm}^{-3}$. La masa crece (decrece) en un $10 \%$ respecto del caso de GR $(\alpha=0)$ para valores de $\alpha=-0.2 \mathrm{~km}^{2}(+0.2$ $\mathrm{km}^{2}$ ), tanto para una densidad central alta o baja.

componente temporal de la métrica, refleja el comportamiento de la presión, mientras que $\Lambda(r)$ es dominada por la masa, y es la masa y no la presión la que requiere evaluaciones de $R_{0}^{\prime \prime}$, y por lo tanto depende explícitamente de las derivadas de primer y segundo orden de la EoS utilizada. 


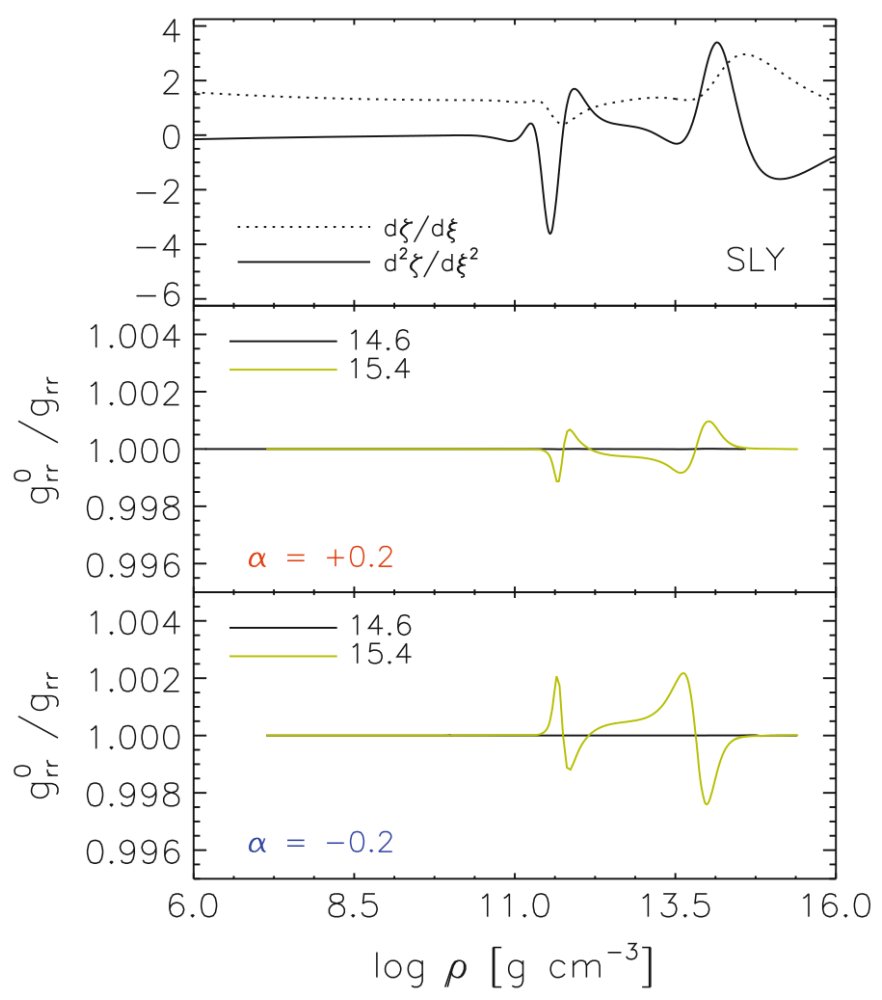

Figura 6.4: Panel superior: perfil de la derivada logarítmica primera (línea punteada) y segunda (línea continua) de la EoS SLY en el interior de la NS. Paneles medio e inferior: perfiles del cociente entre las componentes radiales de la métrica a orden cero (GR), y a primer orden perturbativo $\left(g_{r r}^{0} / g_{r r}\right)$ para $\alpha=+0.2$ y $-0.2 \mathrm{~km}^{2}$, que deberían ser próximos a la unidad como condición necesaria para la validez del método pertubativo. Las desviaciones perturbativas están relacionadas con el comportamiento de la derivada segunda de la EoS.

Para analizar en profundidad el origen de las desviaciones encontradas en las soluciones de gravedad $f(R)$ respecto de la GR, exploramos las contribuciones individuales al gradiente $\mathrm{d} m / \mathrm{d} r$ de los cuatro términos involucrados en la Ecuación 6.10, que llamamos: $4 \pi r^{2} \rho$ (GR) y $A, B, C$ (términos perturbativos). En la Figura 6.5 presentamos la contribución de cada término cuando $\alpha=+0.2 \mathrm{~km}^{2}$ para las EoSs SLY (panel superior izquierdo) y POLY (panel superior derecho), en el caso de una densidad central grande $\left(\log \rho_{c}\left[\mathrm{gr} \mathrm{cm}^{-3}\right]=15.4\right)$. Para la EoS SLY y para valores de la coordenada radial $r \lesssim 9.5 \mathrm{~km}$, el gradiente $\mathrm{d} m / \mathrm{d} r$ está dominado por el término de GR. Más cerca de la superficie de la NS, para $r>9.5 \mathrm{~km}$, el término $C \propto R_{0}^{\prime \prime} / R_{0}$ se torna dominante, provocando la fluctuación en el perfil de masa encerrado. Por el contrario, para la EoS POLY, cuyas derivadas son funciones suaves acotadas muy fuertemente, este efecto antiintuitivo no se observa y la marcha del gradiente $\mathrm{d} m / \mathrm{d} r$ resulta dominada por el término de GR en todo el interior, con pequeñas modificaciones debidas a los términos perturbativos. En los paneles inferiores de la Figura 6.5 presentamos en detalle lo observado en los paneles superiores 

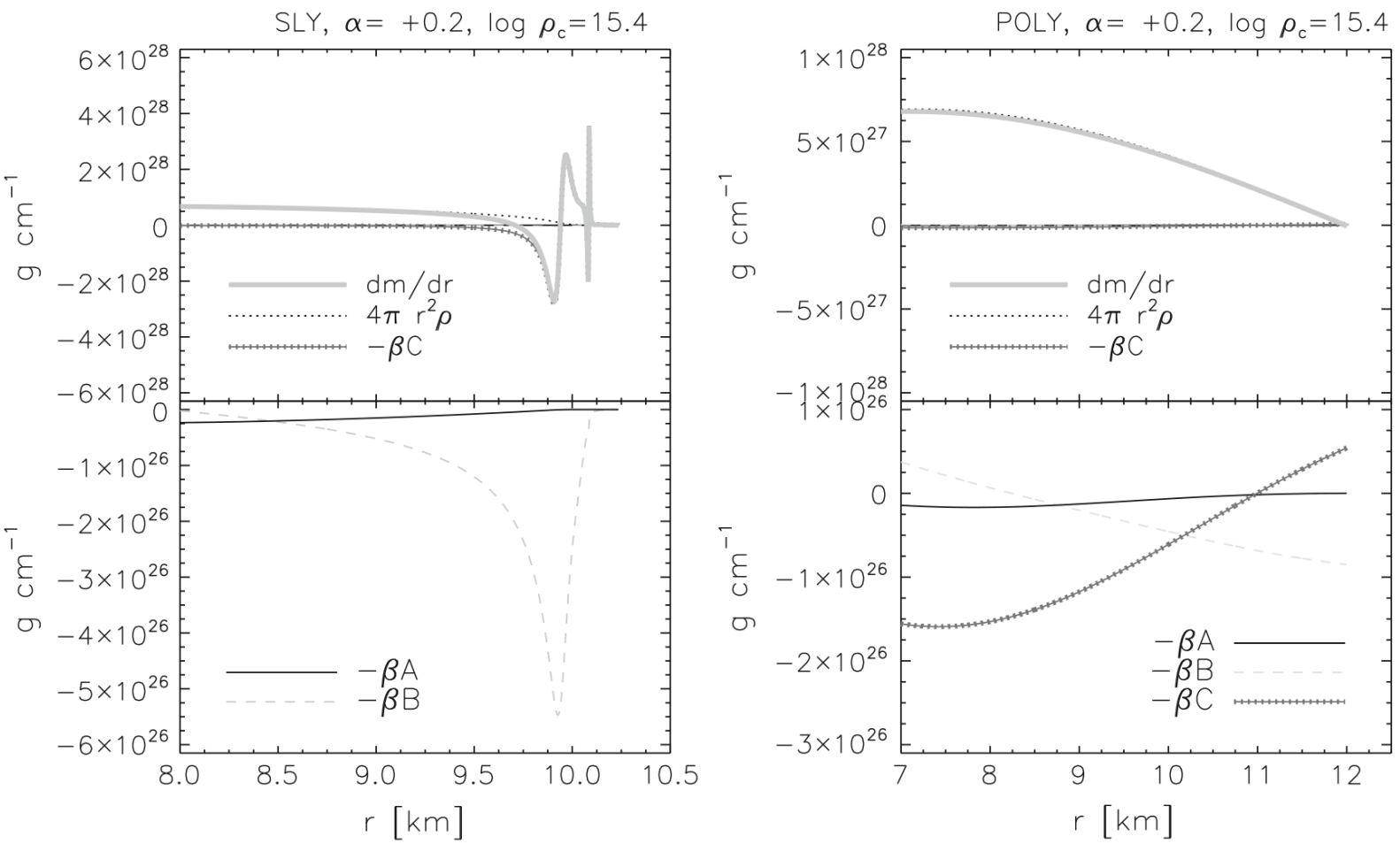

Figura 6.5: Perfil del gradiente de masa encerrada, $\mathrm{d} m / \mathrm{d} r$ (línea gruesa), cerca de la superficie de las NSs para las EoS SLY (panel izquierdo) y POLY (panel derecho). Las desviaciones del perfil de masa del caso de GR son más importantes para las EoS SLY realista. Notar que la línea a trazos indica los términos de orden cero (GR) y la línea continua con signos de suma indica la contribución debida al término $C \propto R_{0}^{\prime \prime} / R_{0}$. Los paneles inferiores muestran el detalle de la contribución de los términos perturbativos menores, indicados en la leyenda.

para mostrar el comportamiento de los términos perturbativos menores.

En la Figura 6.6 extendemos este análisis para comparar el comportamiento de la derivada de la masa encerrada en cuatro casos particulares que involucran a la EoS SLY: $\log \rho_{c}\left[\mathrm{gr} \mathrm{cm}^{-3}\right]=15.4$ y 14.6 y $\alpha=+0.2$ y $-0.2 \mathrm{~km}^{2}$. Para estrellas de densidad central baja, el efecto del término asociado a la derivada segunda $(C)$ es relativamente menor que en el caso de alta densidad central. Las fluctuaciones en este término ocurren en el rango de energía donde la derivada segunda logarítmica de la EoS es relevante, que en este caso corresponde a un rango radial más ancho 7-10.5 km, ya que la densidad cambia más suavemente que en el caso de la estrella con alta densidad central. En este otro caso, la densidad decae abruptamente en un rango estrecho entre los 9.7 y los $10.2 \mathrm{~km}$ que corresponden a la superficie.

En todos los casos que estudiamos, los términos $A$ y $B$, que corresponden a las contribuciones de $\sim R_{0} \mathrm{y} \sim R_{0}^{\prime}$, respectivamente, son varios órdenes de magnitud menores. 

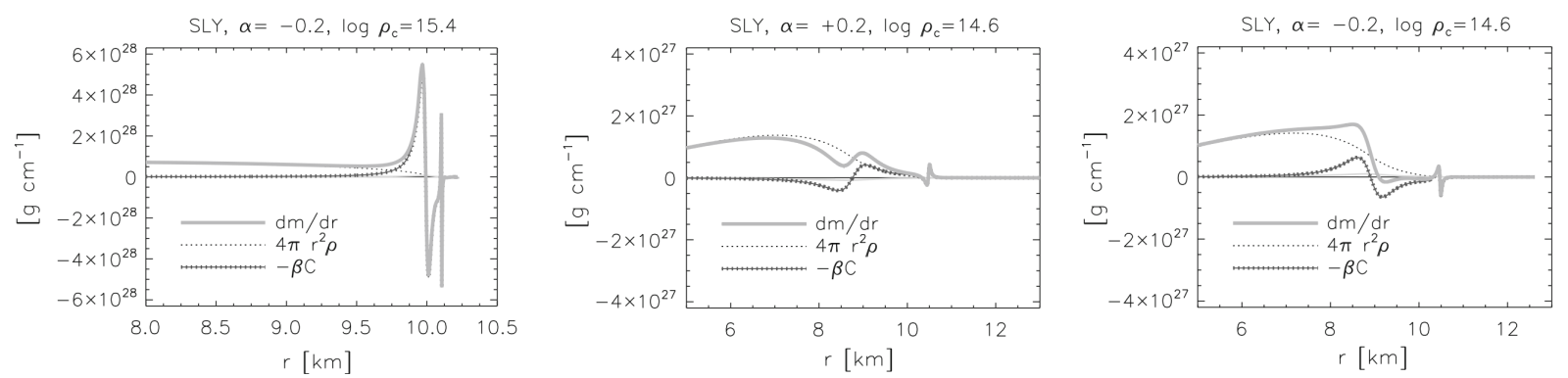

Figura 6.6: Ídem a la Figura 6.5 para otros tres casos usando SLY: $\log \rho_{c}\left[\mathrm{gr} \mathrm{cm}^{-3}\right]=15.4$ (izquierda) y 14.6 (centro y derecha). Los gráficos del centro y de la derecha son una comparación de los efectos introducidos por el cambio de signo en $\alpha$, que está indicado en unidades de $\mathrm{km}^{2}$. Los mismos valores, $\alpha=-0.2$ (izquierda) y +0.2 (Figura 6.5, panel izquierdo) son usados, para una densidad central mayor.

\subsection{Discusión}

Con el objeto de investigar la viabilidad de las teorías de gravedad $f(R)$ para describir escenarios astrofísicos extremos, como los involucrados en las NSs, estudiamos el caso particular de la gravedad cuadrática $f(R)=R+\alpha R^{2}$ usando tanto una EoS realista como una aproximación politrópica. Para ello, seguimos los pasos generales presentados previamente por Cooney et al. (2010) y Arapoğlu et al. (2011) utilizando un método perturbativo para aproximar las ecuaciones de campo de cuarto orden en la métrica. Respecto a las relaciones masa-radio $(M-R)$, encontramos resultados consistentes con estudios previos, hallando que para los valores extremos admitidos para el parámetro libre $\alpha$, estas teorías de gravedad son capaces de incluir soluciones estáticas de mayor masa que la GR. En este sentido, resulta importante remarcar que no existe un acuerdo general acerca del valor de la masa máxima de estos objetos antes de colapsar inevitablemente hacia un agujero negro, dadas las incertezas actuales en el conocimiento del comportamiento de la materia nuclear a las más altas densidades, a través de su EoS. Este problema no puede discernirse en la actualidad dada la carencia de una respuesta final acerca tanto de la microfísica de la materia como del comportamiento de la gravedad en el régimen de campo gravitacional fuerte (Wen et al., 2011).

El resultado más importante que encontramos se concentra entorno a los detalles de la estructura interna de las NSs, en particular, en lo que concierne a los casos asociados a los valores extremos del parámetro $\alpha$ (es decir, las máximas perturbaciones a la GR permitidas por las cotas actuales impuestas en laboratorios y en el Sistema Solar). Encontramos que el comportamiento de la métrica, que en gravedad cuadrática depende no sólo de la EoS, sino también de sus derivadas de orden superior, conlleva la aparición de una región interna en la estrella donde la masa encerrada decrece con el radio.

A pesar del hecho que, en el marco de la GR, este efecto sólo podría ser explicado como producto de la inclusión de un fluido de densidad de energía negativa, en las teorías $f(R)$, éste resultado podría surgir como una consecuencia natural del acoplamiento del espacio-tiempo y 
el contenido de materia, a través de la geometría. Adoptando una EoS politrópica simple, con derivadas de orden superior estrictamente acotadas, dicho comportamiento anómalo en los perfiles internos de las NSs desaparece, y el efecto final producido por la modificación de la gravedad sobre la estructura de estas estrellas se ve reducido fuertemente.

Enfatizamos que las incertezas en la elección de la EoS podrían tener un efecto mayor en las soluciones. Por lo tanto, estos resultados deben ser tomados con cautela, ya que permanece poco claro si los picos en los perfiles internos aparecen aquí como una consecuencia de la representación analítica de la EoS, del esquema perturbativo o de la geometría propia de la teoría de gravedad cuadrática utilizada. En cualquier caso, nuestros resultados imponen nuevos preguntas en el tema. Más investigaciones son necesarias para poder discernir entre estas posibilidades. 


\section{Capítulo 7}

\section{Estrellas de neutrones en teorías $f(R)$ cuadráticas: formalismo de Palatini}

\subsection{Resumen}

Como ya se mencionara en el capítulo anterior, el estudio de la estructura de objetos compactos puede resultar útil para poner cotas a las teorías de gravedad $f(R)$ en el régimen de campo gravitacional fuerte. En este capítulo, analizamos nuevamente las configuraciones de estrellas compactas en teoría cuadrática $f(R)$, pero basándonos en el formalismo de Palatini.

En este contexto, las ecuaciones de campo resultan de segundo orden, como en GR, por lo que las ecuaciones TOV modificadas para una métrica estática y esféricamente simétrica pueden ser derivadas analíticamente en forma exacta. Mostraremos aquí que cuando tales ecuaciones son integradas numéricamente, utilizando una aproximación analítica a una EoS realista, los perfiles internos muestran nuevamente características extrañas, análogo a lo que encontráramos en el formalismo métrico (aunque en ese caso debimos usar un tratamiento perturbativo). Por el contrario, utilizando aproximaciones politrópicas sencillas, estos comportamientos anti-intuitivos en los perfiles de masa encerrada también desaparecen.

Con el objetivo de analizar la correlación entre los perfiles internos y las derivadas de la EoS, proponemos aquí una parametrización basada en dos polítropas para representar el núcleo y la corteza de la NS de manera independiente, lo que nos permite manejar sus derivadas y estudiar en detalle su correlación con los perfiles internos, lo que nos permitió encontrar que es posible obtener NSs más masivas que en GR, únicamente en un caso límite.

\subsection{Introducción}

Como se introdujera en el capítulo anterior, las teorías alternativas a la gravedad son generalizaciones de la GR de Einstein concebidas con la idea de poder poner en acuerdo los problemas teóricos y observacionales de la GR en escenarios cosmológicos (ver Capozziello \& Faraoni, 2011). Una clase particular de estas teorías, las llamadas teorías de gravedad $f(R)$ son obtenidas susti- 
tuyendo la densidad lagrangiana de Einstein-Hilbert por una función del escalar de curvatura de Ricci, $R$. Una de las motivaciones para estudiar tales teorías de gravedad $f(R)$ es la posibilidad de describir las observaciones cosmológicas actuales sin la necesidad de invocar una componente de materia extraña, como la llamada energía oscura en el régimen actual (de baja curvatura) de la evolución del universo (Capozziello \& Faraoni, 2011; de Felice \& Tsujikawa, 2010; Sotiriou, 2006; Sotiriou \& Faraoni, 2010). En este sentido, una serie de modelos $f(R)$ fueron propuestos para dar cuenta estos resultados, satisfaciendo a su vez, las pruebas actuales de la gravedad tanto en el Sistema Solar como en los laboratorios terrestres (Hu \& Sawicki, 2007; Jaime et al., 2011; Miranda et al., 2009; Starobinsky, 2007).

Por el contrario, en el régimen de campo gravitacional fuerte, tanto GR como estas teorías alternativas distan de haber sido restringidas por las observaciones (DeDeo \& Psaltis, 2003). En este contexto, las NSs ofrecen la posibilidad de estudiar en detalle teorías $f(R)$ a través del cálculo de su estructura interna, tanto en el formalismo métrico, como hicimos en el capítulo anterior, como en el de Palatini, que introducimos a continuación (de Felice \& Tsujikawa, 2010).

En el formalismo de Palatini la métrica y la conexión son consideradas entidades geométricas independientes (Olmo, 2011). Este esquema tiene la ventaja de dar como resultado ecuaciones de campo de segundo orden en la métrica (Kainulainen et al., 2007; Olmo, 2008), que admiten soluciones exactas, mientras que, en el formalismo métrico, las ecuaciones diferenciales resultan de cuarto orden, y pueden ser tratadas sólo con métodos perturbativos.

El modelado de NSs en teorías $f(R)$ en el formalismo de Palatini involucra algunas consideraciones adicionales comparadas con el caso de GR, ya que las ecuaciones modificadas de TOV incluyen las derivadas de la EoS adoptada. Debido a esto, Barausse et al. (2008a) mostraron que las soluciones presentan la particularidad que la masa encerrada no resulta una función suave, como en GR, ya que puede desarrollar picos producto de cambios rápidos en las derivadas de la $\mathrm{EoS}^{1}$. A pesar de esto, es importante notar que las derivadas de las EoS están poco restringidas debido a que las EoS son construidas esencialmente para ajustar la relación de orden cero entre $\rho$ y $p$, suficiente para calcular la estructura de las NSs en GR. Es por esto que su extrapolación a usos que involucran derivadas de orden superior ( $\left.\operatorname{como} \mathrm{d} p / \mathrm{d} \rho, \mathrm{d}^{2} p / \mathrm{d} \rho^{2}\right)$, debe tomarse con cierta cautela.

En este capítulo estudiamos el comportamiento de los perfiles de masa encerrada de las soluciones correspondientes a NSs en teorías $f(R)$ bajo el formalismo de Palatini, con el fin de intentar dar respuesta a preguntas abiertas en el capítulo anterior. Allí, estudiando soluciones correspondientes a una teoría cuadrática $f(R)$ bajo el formalismo métrico encontramos que los perfiles internos de la masa encerrada de las soluciones presentaban un comportamiento anómalo

\footnotetext{
${ }^{1}$ Otra característica poco atractiva de las NSs en el formalismo de Palatini, es la aparición de divergencias en el tensor de Riemann y los invariantes de curvatura de la métrica evaluada cerca de la superficie de la estrella, cuando se incorporan EoS politrópicas (Barausse et al., 2008a,b,c; Kainulainen et al., 2007) hecho que fuera reanalizado por Olmo (2008) para el caso particular de gravedad cuadrática. Allí se concluye que la existencia de tales singularidades en la curvatura resulta no-física, ya que surge de manera artificial producto de la EoS idealizada que se utiliza para calcular la estructura de la estrella. Además, admitiendo funciones $f(R)$ continuas pero no analíticas, en Mana et al. (2015) fue demostrado que el intervalo de parámetros en que no se forma una singularidad puede extenderse para cubrir casos físicamente relevantes que fueron previamente ignorados.
} 
o anti-intuitivo. Discutiremos entonces acerca de si se trata de un subproducto de las aproximaciones analíticas a las EoS utilizadas o de una propiedad intrínseca de estas teorías de gravedad alternativas.

Para ello, calculamos la estructura de una estrella en la teoría definida por $f(R)=R+\alpha R^{2}$ (también usada en Barausse et al., 2008a) de dos maneras diferentes. Primero, usando la EoS realista SLY (en lugar de la EoS FPS usada en Barausse et al., 2008a), que ya usamos en el capítulo anterior. Luego, a través de la incorporación de una aproximación a la EoS basada en la conexión de dos polítropas diferentes. Estas polítropas representan el estado de la materia en el núcleo y en la corteza de la NS, respectivamente, permitiéndonos a su vez manejar sus derivadas a través de un conjunto de parámetros que facilitan el análisis de su correlación con los perfiles internos correspondientes. Encontramos que en ambos casos los perfiles internos se encuentran fuertemente correlacionados con las derivadas de la EoS, dando lugar a regiones donde la masa encerrada decrece con la coordenada radial de manera anti-intuitiva. La única excepción a este comportamiento es obtenida para una elección específica de los parámetros de la polítropa múltiple. Siguiendo el trabajo de Barausse et al. (2008a), exploramos además la familia de configuraciones masa-radio $(M-R)$ para $f(R)=R+\alpha R^{2}$ en el formalismo de Palatini. Nuestros resultados muestran que las NSs del modelo elegido son menos masivas que las de GR, excepto para el caso particular mencionado, en el que la masa crece con el valor de $\alpha$.

\subsection{Ecuaciones de la estructura estelar}

Como se introdujera en el Capítulo 1, en esta teoría la acción modificada de Einstein-Hilbert viene dada por

$$
S\left[g_{\mu \nu}, \Gamma, \psi_{\mathrm{m}}\right]=\frac{c^{4}}{16 \pi G} \int \mathrm{d}^{4} x \sqrt{-g} f(R)+S_{\mathrm{m}}\left[g_{\mu \nu}, \psi_{\mathrm{m}}\right]
$$

donde $f(R)$ es una función del escalar de Ricci, $R \equiv g^{\mu \nu} R_{\mu \nu}(\Gamma)$, con $R_{\mu \nu}(\Gamma)=-\partial_{\mu} \Gamma_{\mu \nu}^{\lambda}+\partial_{\lambda} \Gamma_{\mu \nu}^{\lambda}+\Gamma_{\mu \nu}^{\lambda} \Gamma_{v \lambda}^{\rho}-$ $\Gamma_{\nu \rho}^{\lambda} \Gamma_{\mu \lambda}^{\rho}$. La acción de la materia $S_{\mathrm{m}}$ depende en los campos de materia $\psi_{\mathrm{m}}$ y la métrica $g_{\mu \nu}$.

En el formalismo de Palatini, las ecuaciones de campo se obtienen variando la acción respecto de la métrica y la conexión afín (Olmo, 2008),

$$
\begin{aligned}
f_{R}(R) R_{\mu \nu}(\Gamma)-\frac{1}{2} f(R) g_{\mu \nu} & =\frac{8 \pi G}{c^{4}} T_{\mu \nu} \\
\nabla_{\rho}\left[\sqrt{-g}\left(\delta_{\lambda}^{\rho} f_{R} g^{\mu \nu}-\frac{1}{2} \delta_{\lambda}^{\mu} f R g^{\rho \nu}-\frac{1}{2} \delta_{\lambda}^{v} f_{R} g^{\mu \rho}\right)\right] & =0
\end{aligned}
$$

donde $f_{R} \equiv \mathrm{d} f / \mathrm{d} R$ y $T_{\mu \nu}$ es el tensor de energía y momento, que satisface la ecuación de continuidad

$$
\nabla_{\mu} T^{\mu \nu}=0
$$

La traza de la Ecuación 7.2 resulta

$$
f_{R}(R) R-2 f(R)=\frac{8 \pi G}{c^{4}} T
$$


Esta ecuación algebraica puede utilizarse para expresar el escalar de curvatura, $R$, como función de la traza del tensor de energía y momento, $T$.

La estructura estelar se calcula suponiendo una métrica estática y esféricamente simétrica con un elemento de línea dado por

$$
\mathrm{d} s^{2}=-e^{A(r)} c^{2} \mathrm{~d} t^{2}+e^{B(r)} \mathrm{d} r^{2}+r^{2}\left(\mathrm{~d} \theta^{2}+\sin ^{2} \theta \mathrm{d} \phi^{2}\right),
$$

y un fluido ideal con un tensor de energía e impulso $T_{\mu \nu}=\left(c^{2} \rho+p\right) u_{\mu} u_{v}+p g_{\mu \nu}$, donde $\rho(r)$ es el perfil de densidad, y $p(r)$ es el perfil de la presión. Con estas consideraciones, la ecuación de continuidad (7.4) resulta

$$
p^{\prime}=-\frac{A^{\prime}}{2}\left(c^{2} \rho+p\right)
$$

y las componentes de las ecuaciones de campo, $t$ y $r r$, pueden escribirse como (Barausse et al., 2008a; Reijonen, 2009):

$$
\begin{aligned}
A^{\prime} & =-\frac{1}{1+\gamma_{0}}\left(\frac{1-e^{B}}{r}-\frac{e^{B}}{F} \frac{8 \pi G r p}{c^{4}}+\frac{\alpha_{0}}{r}\right) \\
B^{\prime} & =\frac{1}{1+\gamma_{0}}\left(\frac{1-e^{B}}{r}+\frac{e^{B}}{F} \frac{8 \pi G r \rho}{c^{2}}+\frac{\alpha_{0}+\beta_{0}}{r}\right)
\end{aligned}
$$

donde usamos primas para notar las derivadas con respecto a la coordenada radial, $r, \mathrm{y}$

$$
\begin{aligned}
\alpha_{0} & \equiv r^{2}\left(\frac{3}{4}\left(\frac{f_{R}^{\prime}}{f_{R}}\right)^{2}+\frac{2 f_{R}^{\prime}}{r f_{R}}+\frac{e^{B}}{2}\left(R-\frac{f}{f_{R}}\right)\right) \\
\beta_{0} & \equiv r^{2}\left(\frac{f_{R}^{\prime \prime}}{f_{R}}-\frac{3}{2}\left(\frac{f_{R}^{\prime}}{f_{R}}\right)^{2}\right) \\
\gamma_{0} & \equiv \frac{r f_{R}^{\prime}}{2 f_{R}}
\end{aligned}
$$

Las ecuaciones TOV modificadas toman entonces la forma (Kainulainen et al., 2007; Reijonen, 2009)

$$
\begin{aligned}
\frac{\mathrm{d} p}{\mathrm{~d} r} & =-\frac{1}{1+\gamma_{0}} \frac{c^{2} \rho+p}{r\left(c^{2} r-2 G m\right)}\left(G m+\frac{4 \pi G r^{3} p}{f_{R}}-\frac{\alpha_{0}}{2}\left(c^{2} r-2 G m\right)\right) \\
\frac{\mathrm{d} m}{\mathrm{~d} r} & =\frac{1}{1+\gamma_{0}}\left(\frac{4 \pi r^{2} \rho}{f_{R}}+\frac{c^{2}}{G} \frac{\alpha_{0}+\beta_{0}}{2}-\frac{m}{r}\left(\alpha_{0}+\beta_{0}-\gamma_{0}\right)\right)
\end{aligned}
$$

donde $m(r) \equiv c^{2} r\left(1-e^{-B}\right) / 2 G$. A partir de aquí, elegimos la forma particular para la $f(R)$, caracterizada por la función $f(R)=R+\alpha R^{2}$. $^{2}$

La constante $\alpha$ es un parámetro libre de la teoría que debe ser positivo debido a consideraciones de estabilidad (de Felice \& Tsujikawa, 2010; Sotiriou \& Faraoni, 2010). Este tipo de teoría ha sido estudiada ampliamente debido a sus propiedades de renormalización (Stelle, 1977). También

\footnotetext{
${ }^{2}$ Las potencias negativas de $R$ son despreciables en el régimen de campo gravitacional fuerte, que es el límite en el que estamos interesados para modelar NSs Sotiriou (2006).
} 
fueron estudiados agujeros negros cargados en teorías $f(R)=R+\alpha R^{2}$ bajo el formalismo de Palatini (Olmo \& Rubiera-Garcia, 2013) así como modelos cosmológicos con rebote (Barragán et al., 2009).

El sistema de ecuaciones diferenciales formado por 7.13 y 7.14 puede resolverse si se explicita una relación entre $\rho$ y $p$, la EoS. Usando la Ecuación 7.5, el escalar de curvatura, $R$, puede expresarse como función de la traza $T$. En particular, la gravedad cuadrática da como resultado $R=-8 \pi G T / c^{4}=-8 \pi G\left(-c^{2} \rho+3 p\right) / c^{4}$. A través de la regla de la cadena, pueden calcularse las derivadas $f_{R}$ respecto a la coordenada radial, $r$, a través de las funciones $\alpha_{0}, \beta_{0}$ y $\gamma_{0}$, que quedan entonces escritas en función de $p^{\prime}, p^{\prime \prime}$, y las derivadas primera y segunda de la EoS $(\mathrm{d} \rho / \mathrm{d} p$ y $\left.\mathrm{d}^{2} \rho / \mathrm{d} p^{2}\right)$. Luego, el cálculo de la estructura estelar requiere una derivación de las ecuaciones 7.13 y 7.14 explícita que resulta no trivial (Reijonen, 2009).

\subsection{Ecuación de estado}

Como se mencionara previamente, la EoS contiene información de las condiciones microfísicas de la materia en el interior de las NSs a lo largo de varios órdenes de magnitud en la densidad.

Las EoS disponibles en la literatura están usualmente expresadas a través de tablas donde se explicita la relación de orden cero entre $\rho$ y $p$, ya que esa es la relación necesaria para calcular la estructura de NSs en GR. Sin embargo, en al ser necesario el cálculo de las derivadas de la EoS, como en nuestro caso, las técnicas usuales de interpolación no son suficientes, ya que fallan a la hora de representar adecuadamente las derivadas de orden superior Ekşi et al. (2014). Es por esto que las aproximaciones analíticas a las EoS disponibles en la literatura resultan preferibles para obtener derivadas como $\mathrm{d} p / \mathrm{d} \rho$ y $\mathrm{d}^{2} p / \mathrm{d} \rho^{2}$, como en el caso que nos interesa aquí. En este sentido, consideramos primero la EoS SLY realista presentada en el Capítulo 6, que ha sido ampliamente utilizada para calcular tanto la estructura interna de NSs (Douchin \& Haensel, 2001; Haensel \& Potekhin, 2004) como modelos de enfriamiento y evolución magnética (Aguilera et al., 2009; García et al., 2014), así como la EoS FPS, usada por Barausse et al. (2008a) a modo de comparación. Estas aproximaciones son representaciones polinómicas complejas que ajustan a la relación dada por la tabla asociada a cada EoS, mediante un procedimiento termodinámicamente consistente tanto en los regímenes de densidad correspondientes a la corteza como al núcleo (Haensel \& Potekhin, 2004).

Como ya mostramos en el capítulo anterior, las parametrizaciones analíticas para las EOSs SLY y FPS están dadas por

$$
\begin{aligned}
\zeta= & \frac{a_{1}+a_{2} \xi+a_{3} \xi^{3}}{1+a_{4} \xi} f_{0}\left(a_{5}\left(\xi-a_{6}\right)\right)+\left(a_{7}+a_{8} \xi\right) f_{0}\left(a_{9}\left(a_{10}-\xi\right)\right) \\
& +\left(a_{11}+a_{12} \xi\right) f_{0}\left(a_{13}\left(a_{14}-\xi\right)\right)+\left(a_{15}+a_{16} \xi\right) f_{0}\left(a_{17}\left(a_{18}-\xi\right)\right),
\end{aligned}
$$

donde $\xi=\log \left(\rho / \mathrm{g} \mathrm{cm}^{-3}\right), \zeta=\log \left(p / \mathrm{dyn} \mathrm{cm}^{-2}\right), f_{0}(x)=\frac{1}{\mathrm{e}^{x}+1}$, y los coeficientes $a_{i}$ para cada caso están tabulados en Haensel \& Potekhin (2004). Las aproximaciones analíticas a las EoS SLY y FPS se muestran en la Figura 7.1, así como sus derivadas logarítmicas primera y segunda. Incluimos además, a modo de comparación, la EoS dada por la aproximación politrópica del 


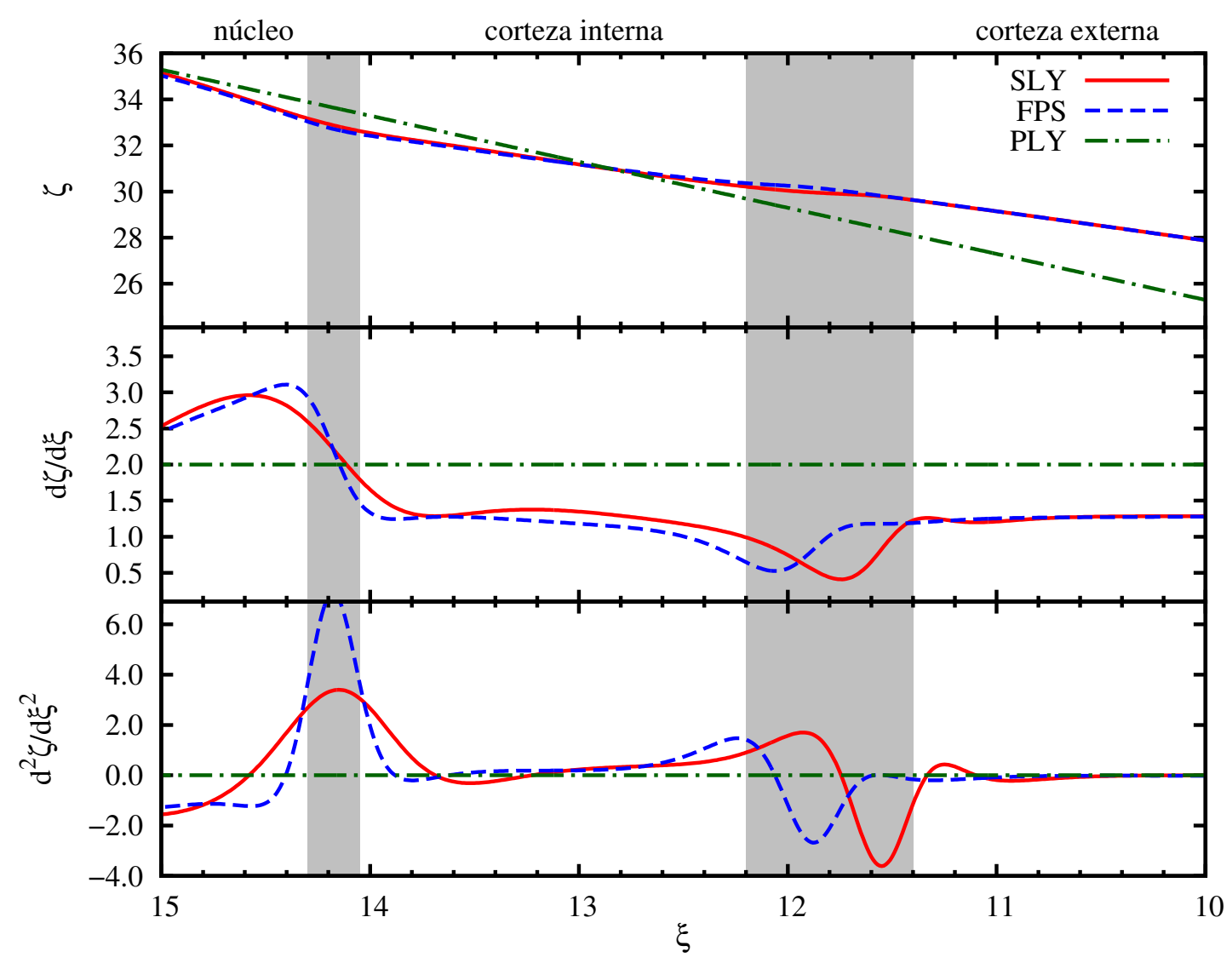

Figura 7.1: Aproximación analítica de las EoSs SLY y FPS (panel superior), y sus derivadas primera y segunda (paneles medio e inferior, respectivamente). Se incluye además la aproximación politrópica simple PLY para comparación. Las áreas sombreadas en color gris indican las regiones de transición entre las distintas regiones internas de las NSs.

capítulo anterior (Silbar \& Reddy, 2004), que llamamos PLY, dada por $\zeta=2 \xi+5.29355$. A pesar que esta última no es una EoS realista que pueda representar una NS en detalle, resulta un modelo simple que nos permite realizar un análisis detallado de rol de las derivadas de las EoSs en el cálculo de la estructura estelar en gravedad $f(R)$.

\subsection{Método numérico}

La resolución del sistema de ecuaciones diferenciales ordinarias formado por 7.13 y 7.14 requiere de su integración desde el centro de la NS hasta su superficie, para lo cual es necesario suponer las condiciones de borde: $m(r=0)=0, p(r=0)=p_{c}, \rho(r=0)=\rho_{c}, p(r=R)=10^{-12} p_{c}$ y $m(r=R)=M$. Para realizar la integración numérica utilizamos un código basado en el método de Runge-Kutta de cuarto orden con un paso variable en la coordenada radial que se acorta 
sistemáticamente al acercarse a la superficie de la NS y que da cuenta de las variaciones rápidas de los parámetros físicos en esta región.

\subsection{Resultados}

En la Figura 7.2, mostramos la familia de configuraciones estáticas para las EoSs SLY, FPS y PLY, para tres valores diferentes del parámetro $\alpha$, que van desde $\alpha=0$ (caso GR) hasta $\alpha=$ $5 \times 10^{-9} \mathrm{~cm}^{2}$. Mientras que las diferencias entre la gravedad modificada y la GR no son apreciables para la EoS PLY, al incluir EoSs realistas, los cambios se tornan significativos. Utilizando las mismas condiciones iniciales para realizar la integración, la masa de la configuración más masiva de cada familia decrece cuando el parámetro $\alpha$ se incrementa. Esto significa que para estar elección particular de la teoría de gravedad modificada, considerando $\alpha>0$, para ambas EoS realistas, no son admisibles NSs más masivas que en GR. Esta característica de las NSs en el modelo $R+\alpha R^{2}$ estaría en conflicto con la evidencia observacional reciente en que se han reportado NSs como PSR J1614-2230 con $M=1.97 \pm 0.04 \mathrm{M}_{\odot}$ (Demorest et al., 2010) y PSR J0348+0432 de $M=2.01 \pm 0.04 \mathrm{M}_{\odot}$ (Antoniadis et al., 2013).

En la Figura 7.3, pueden observarse particularidades más notorias en los perfiles internos de la masa obtenidos usando la EoS SLY para $\rho_{c}=4 \times 10^{15} \mathrm{gr} \mathrm{cm}^{-3}$ y tres valores del parámetro $\alpha$. En ellos se muestran también las derivadas de la EoS usada a modo de comparación.

Allí puede verse que existen regiones internas donde la masa encerrada decrece con $\rho$, es decir que $\mathrm{d} m / \mathrm{d} \rho<0, \mathrm{y}$, como la densidad y la presión decrecen monótonamente con la coordenada radial, en dichas regiones, la masa decrece entonces con la coordenada radial $(\mathrm{d} m / \mathrm{d} r<0)$. Este comportamiento inesperado se torna más pronunciado cuando $\alpha$ crece. Además, las regiones en que estos rasgos son observados están en clara correlación con los intervalos en los que la derivada segunda de la EoS se torna importante, o sea, cerca de las regiones donde ocurren las transiciones de fase, en particular, en la transición entre la corteza y el núcleo, entorno a $\xi=14.1$. Esta correlación se evidencia por el hecho de que $\mathrm{d} m / \mathrm{d} \rho$ se mantiene positiva cuando utilizamos la EoS PLY que posee derivadas triviales. Este comportamiento anti-intuitivo fue reportado previamente por Barausse et al. (2008a) usando la EoS FPS. En este estudio los autores argumentan que estos efectos deberían aparecen en cualquier teoría que involucre derivadas de mayor orden en los campos de materia que en la métrica, ya que en tales teorías no está garantizada la dependencia acumulativa de la métrica respecto de los campos de materia. De hecho, este comportamiento resulta análogo al encontrado en el estudio de la estructura de NSs bajo el formalismo métrico, como vimos en el capítulo anterior. Cabe destacar que en ese caso, como las ecuaciones son de cuarto orden, se requirió la incorporación de un método perturbativo para su cálculo, mientras que en este caso, estamos tratando con soluciones exactas.

Este escenario motiva una análisis cuidadoso de las aproximaciones numéricas a las EoS realistas utilizadas, ya que, generalmente, estas EoSs están construidas con el objeto de construir NSs en GR. Tal procedimiento podría dar lugar a la pérdida de información importante contenida en las derivadas primera y segunda de la EoS, que se tornan relevantes en gravedad $f(R)$. Es por esto que vale la pena investigar otras descripciones de NSs realistas de forma tal que las derivadas de 


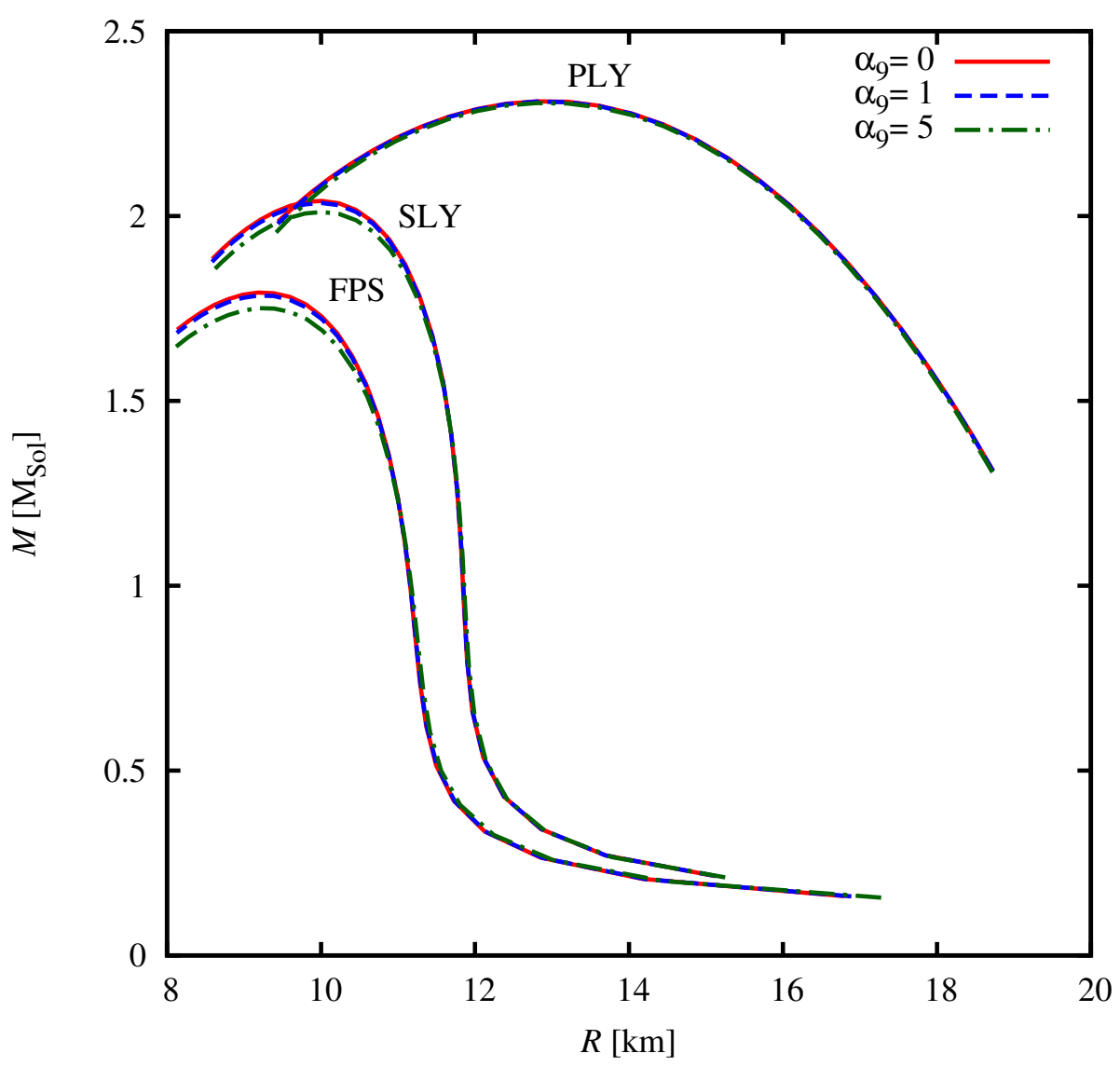

Figura 7.2: Configuraciones estáticas para las EoSs SLY, FPS y PLY, considerando distintos valores del parámetro $\alpha$. El caso de GR se recupera cuando $\alpha=0$, para el cual se obtienen las masas máximas en todos los casos.

la EoS permanezcan bajo control. En la Sección que sigue presentamos una primera aproximación a tal problema, proponiendo una parametrización alternativa para imitar el comportamiento de las EoSs cerca de las regiones de transición de fase. Veremos que tal parametrización ofrece una descripción simplificada de la materia nuclear en la corteza y el núcleo de una NS, permitiéndonos a su vez jugar con diferentes valores para $\mathrm{d} p / \mathrm{d} \rho$ y $\mathrm{d}^{2} p / \mathrm{d} \rho^{2}$ que nos permitirán volver a interpretar los resultados presentados hasta aquí.

\subsection{Una parametrización alternativa}

El interior de las NSs suele ser bien descripto por tres regiones diferentes: el núcleo, la corteza interna y la externa (ver Figura 7.1). Cada una de estas regiones puede aproximarse por una EoS politrópica con un índice característico $\Gamma=\mathrm{d} \log p / \mathrm{d} \log \rho=\mathrm{d} \zeta / \mathrm{d} \xi$ (Read et al., 2009). Restringiremos nuestro análisis a las densidades que cubren el núcleo y la corteza interna, así como la región de transición correspondiente $(\xi \sim 14.2)$, donde el perfil de masa presenta las diferencias 


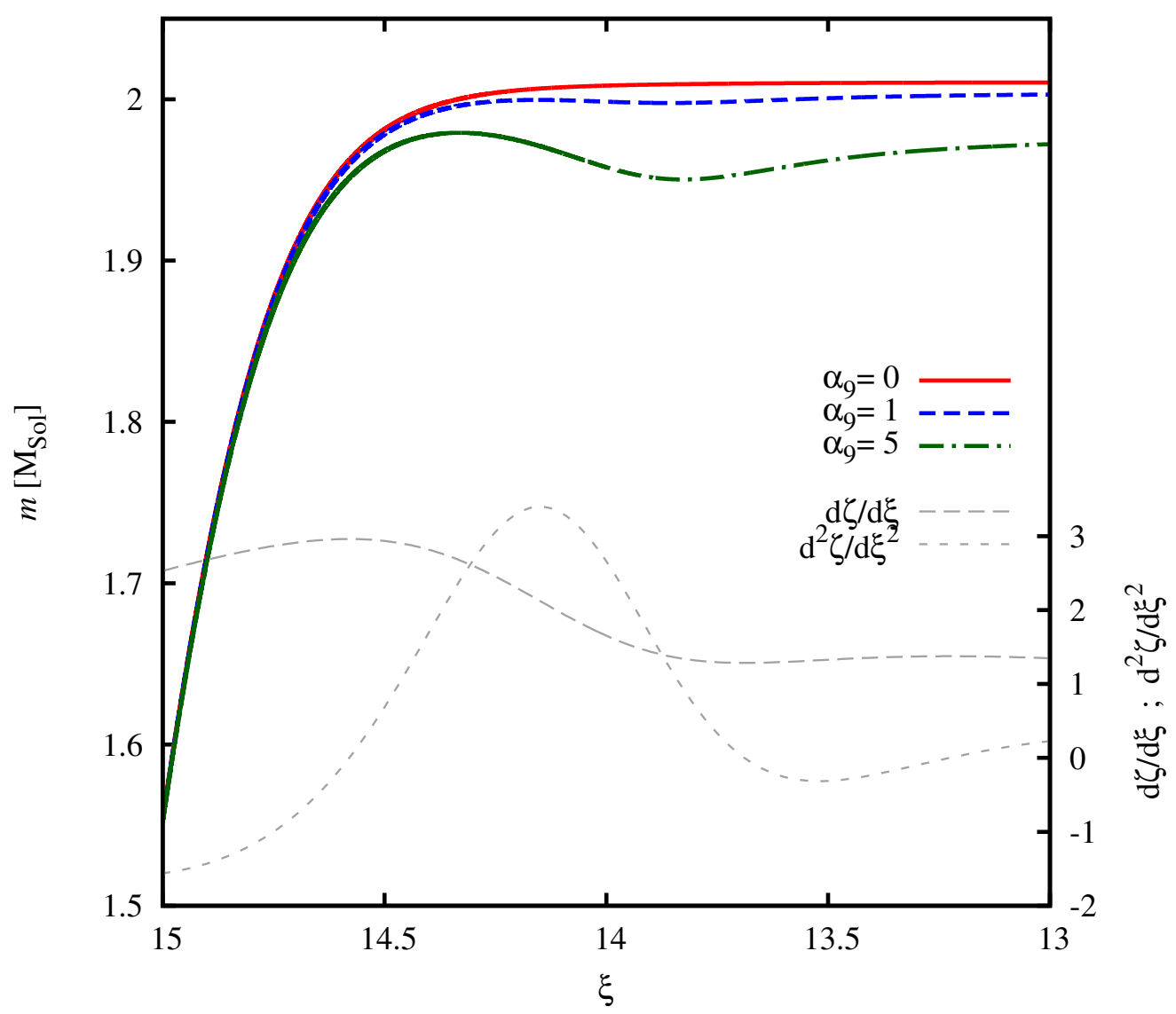

Figura 7.3: Perfil de masa encerrada para la región de transición entre el núcleo y la corteza interna usando la EoS SLY para $\rho_{c}=4 \times 10^{15} \mathrm{gr} \mathrm{cm}^{-3}$. El comportamiento anti-intuitivo $\mathrm{d} m / \mathrm{d} \xi<0$ es más notorio cuando $\alpha$ crece. Las derivadas primera y segunda de la EoS SLY se grafican en líneas de trazos grises para evidenciar las fuertes correlaciones entre los perfiles de masa y la derivada segunda de la EoS, que se torna prominente en la región de transición entre el núcleo y la corteza $(\xi \sim 14.0-14.3)$.

más significativas respecto del caso de GR (ver Figura 7.3).

Elegimos representar esta descripción por una EoS arbitraria que denominamos PLYT, que presentamos en la Figura 7.4, junto a sus derivadas primera y segunda. En esta representación, sus derivadas logarítmicas vienen dadas por

$$
\begin{aligned}
\frac{\mathrm{d} \zeta}{\mathrm{d} \xi} & =\frac{\Gamma_{2}-\Gamma_{1}}{\pi} \tan ^{-1}\left(\frac{\xi-\xi_{0}}{\Delta}\right)+\frac{\Gamma_{1}+\Gamma_{2}}{2} \\
\frac{\mathrm{d}^{2} \zeta}{\mathrm{d} \xi^{2}} & =\frac{\Gamma_{2}-\Gamma_{1}}{\pi} \frac{\Delta}{\Delta^{2}+\left(\xi-\xi_{0}\right)^{2}}
\end{aligned}
$$

donde $\Gamma_{2}$ y $\Gamma_{1}$ son los índices politrópicos en el núcleo y la corteza interna, respectivamente. El parámetro $\Delta$ caracteriza el ancho de la región de transición, permitiéndonos controlar las derivadas 
primera y segunda de la EoS PLYT. Debido a que las derivadas segundas no son restringidas por la termodinámica, $\Delta$ puede reducirse arbitrariamente. ${ }^{3}$ En el caso límite en que ambas EoS politrópicas son pegadas con $\Delta=0$, el valor de la densidad $\xi_{0}$ indica el punto de transición entre el núcleo y la corteza interna, dando $\zeta(\xi)$ continua pero no diferenciable en el punto $\xi_{0}$. La forma explícita de la EoS PLYT puede obtenerse integrando la Ecuación 7.16.

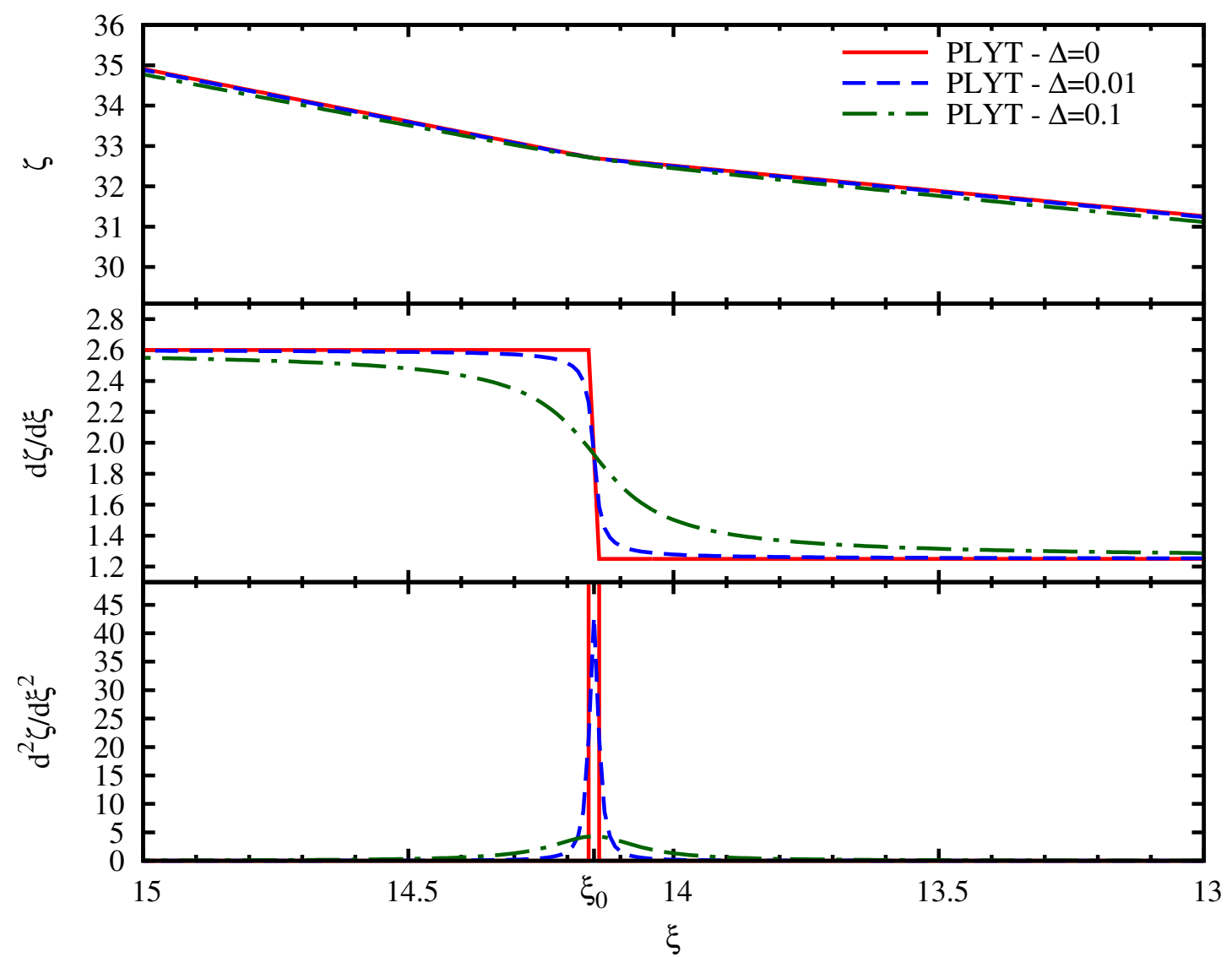

Figura 7.4: EoSs PLYT alrededor de la transición entre la corteza y el núcleo $\xi \sim 14.1(\xi \sim 11.9)$. Para imitar a la EoS SLY en el rango $13 \leq \xi \leq 15$, fijamos $\Gamma_{2}=2.6, \Gamma_{1}=1.25, \xi_{0}=14.15 \mathrm{y}$ $\zeta\left(\xi_{0}=14.15\right)=32.7$. Usamos $\Delta=0,0.1,0.01$ como ejemplos ilustrativos.

En la Figura 7.5 mostramos las configuraciones masa-radio y los perfiles internos obtenidos usando la EoS PLYT. Para imitar la EoS SLY en el rango en que estamos interesados, $13 \leq \xi \leq 15$, fijamos $\Gamma_{2}=2.6, \Gamma_{1}=1.25, \xi_{0}=14.15$ y $\zeta_{0}\left(\xi_{0}=14.15\right)=32.7$ en las ecuaciones 7.16 y 7.17 . Usamos $\Delta=0.1,0.01$ como ejemplos que permiten reproducir resultados similares a los obtenidos usando la EoS realista SLY en la Sección anterior. Las configuraciones masa-radio alcanzan masas máximas menores a medida que el parámetro $\alpha$ se incrementa, y el comportamiento peculiar $\mathrm{d} m / \mathrm{d} \xi<0$

\footnotetext{
${ }^{3}$ Ver Bejger et al. (2005) para una discusión acerca de los escenarios astrofísicos para la formación de fases mixtas en el núcleo de las NSs.
} 
puede observarse en los perfiles internos, siendo más pronunciado para valores menores de $\Delta$. Sin embargo, encontramos un comportamiento completamente diferente en el caso límite en que $\Delta=0$, para el cual $\mathrm{d} \zeta / \mathrm{d} \xi$ y d $\mathrm{d}^{2} \zeta / \mathrm{d} \xi^{2}$ no son continuas en el punto de transición $\xi_{0}$. A pesar de estas discontinuidades, el cálculo de la estructura estelar no conlleva problemas numéricos al realizar la integración desde el núcleo hacia la corteza. Por el contrario de los casos previamente analizados, al incrementar $\alpha$ las configuraciones masa-radio halladas permiten albergar NSs más masivas que la GR. Esta característica está en correlación con los perfiles de masa, que se incrementan monótonamente con $\xi$. Luego, el caso $\Delta=0$ no sólo reconcilia las configuraciones masa-radio con los datos observacionales, sino que remueve el comportamiento anti-intuitivo $\mathrm{d} m / \mathrm{d} \xi<0$ de los perfiles de masa.
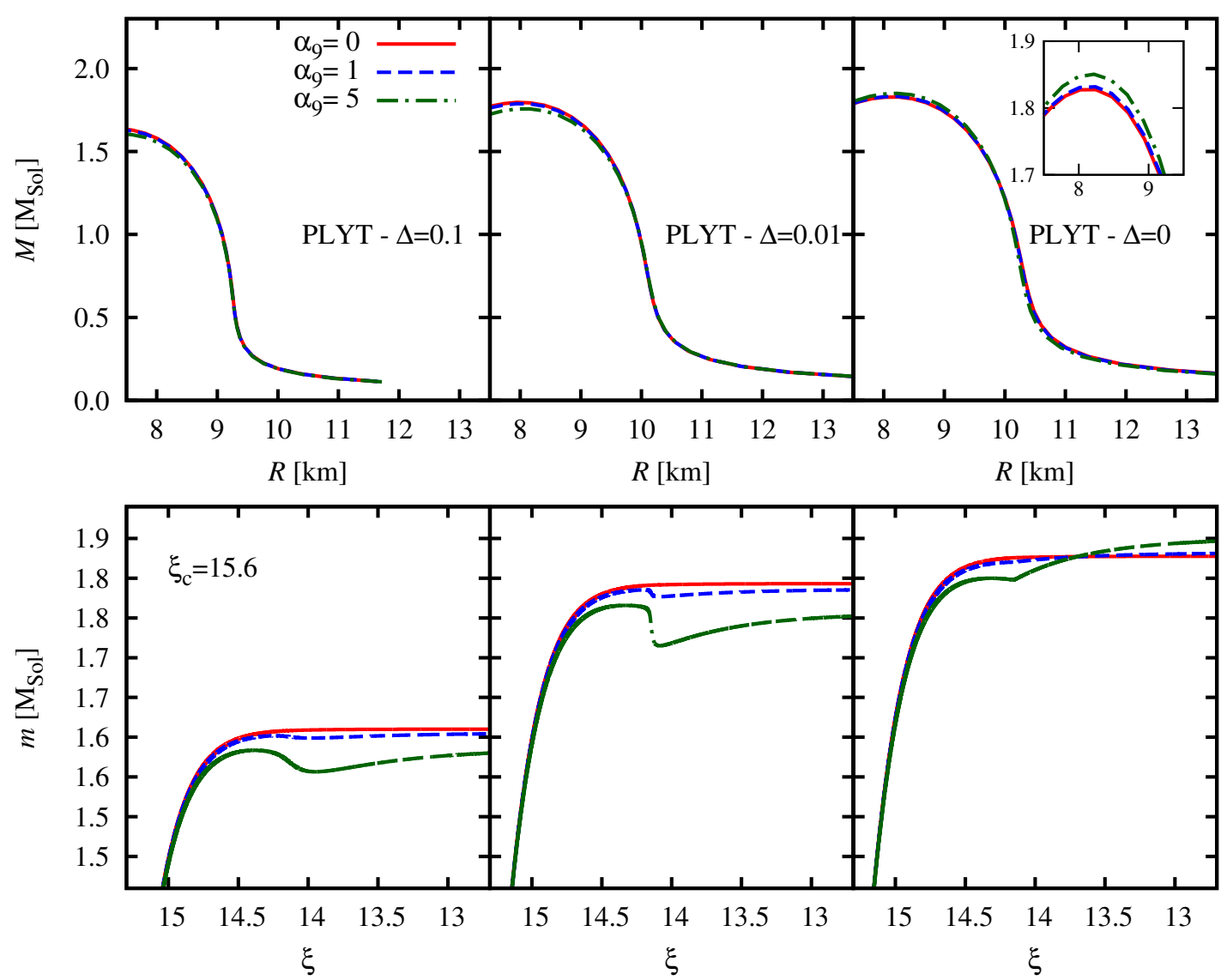

Figura 7.5: Configuraciones masa-radio estáticas (paneles superiores) y perfiles de masa encerrada (paneles inferiores) para la EoS PLYT, considerando distintos valores del parámetro $\alpha$, y, de izquierda a derecha, tres valores diferentes del ancho de la región de transición $(\Delta=0.1,0.01$ y 0$)$. El caso de GR es recuperado con $\alpha=0$. Los perfiles de masa encerrada corresponden a $\rho_{c}=4 \times 10^{15} \mathrm{gr} \mathrm{cm}^{-3}$. El comportamiento anti-intuitivo $\mathrm{d} m / \mathrm{d} \xi<0$ ocurre para todos los casos en que $\Delta \neq 0$, en la región de transición $(\xi=14.15)$. 


\subsection{Discusión}

Con el fin de investigar si teorías $f(R)$ bajo el formalismo de Palatini pueden describir adecuadamente escenarios astrofísicos en el régimen de campo gravitacional fuerte, estudiamos la estructura interna de NSs en gravedad cuadrática, definida por $f(R)=R+\alpha R^{2}$. Al contrario de lo que ocurre en el formalismo métrico, las ecuaciones TOV modificadas resultan de segundo orden, como en el caso de la GR, lo que permite su resolución de manera exacta. Sin embargo, a pesar de esta ventaja, su integración involucra consideraciones adicionales ya que las derivadas de la EoS impactan explícitamente sobre las ecuaciones de estructura.

Considerando la EoS SLY utilizada comúnmente para el cálculo de NSs en GR, obtuvimos resultados consistentes con estudios previos (Barausse et al., 2008a, en donde se usó la EoS FPS) en cuanto a las configuraciones masa-radio estáticas y los perfiles internos de masa encerrada. En cuanto a las relaciones masa-radio, en todos los casos encontramos que las masas máximas admitidas resultan menores al caso de GR, por lo cual, el parámetro $\alpha$ resulta fuertemente restringido dada la evidencia observacional provista por la detección de NSs con masas $M \sim 2 \mathrm{M}_{\odot}$. Pero un problema incluso más serio es encontrado al analizar los perfiles internos de la estructura de estas soluciones. Un comportamiento anti-intuitivo es observado en los perfiles de masa encerrada, que incluyen, regiones donde $\mathrm{d} m / \mathrm{d} \rho<0$. En Barausse et al. (2008a) se argumenta que este tipo de rasgos de los perfiles surgen como consecuencia natural en teorías de gravedad que involucran derivadas de orden superior en los campos de materia que en la métrica. Sin embargo, las EoSs para la materia en el régimen de densidades extremas suelen ser acotadas a través del ajuste de la estructura de las NSs calculadas a partir de ellas, bajo la teoría de GR, donde únicamente es relevante la relación de orden cero entre $\rho$ y $p$. Luego, puede resultar poco apropiado, utilizar estas EoSs para restringir las teorías alternativas a la gravedad sin imponer la tendencia a que $\alpha=0$. Esto es por cierto un problema intrincado ya que justamente son las NSs los laboratorios naturales donde pueden ser estudiadas las propiedades de la materia a alta densidad.

Además, en este trabajo se estudió una parametrización alternativa para las EoSs que denominamos PLYT, que da cuenta de manera simple de la región de transición entre la corteza y el núcleo de NSs. Para esto utilizamos aproximaciones politrópicas a cada región incorporando una función analítica para parametrizar e imitar la transición de fase entre ellas, de manera de poder controlar las derivadas primera y segunda. Usando esta EoS simple, fuimos capaces de recuperar las configuraciones masa-radio obtenidas usando aproximaciones analíticas a las EoS realistas como SLY y FPS, así como sus perfiles interiores, hallando además un resultado notorio en el caso límite que representa una transición de fase dura entre el núcleo y la corteza de la estrella compacta. En este caso, las soluciones resultan completamente diferentes. No sólo encontramos NSs más masivas que las admitidas por la GR para estas EoS, sino que los comportamiento anti-intuitivos de los perfiles internos fueron removidos. Estos resultados parecen sugerir que los problemas que fueron mencionados como característicos de las teorías $f(R)$ bajo el formalismo de Palatini, podrían estar relacionados con las incertezas que surgen de la microfísica asociada a la transición de fase a alta densidad. 


\section{Parte V}

\section{Conclusiones y perspectivas a futuro}





\section{Capítulo 8}

\section{Conclusiones y desarrollos a futuro}

En esta tesis hemos realizado aportes al conocimiento de las NSs tanto desde un punto de vista teórico como del análisis de datos surgidos de observaciones usando diferentes enfoques y herramientas. En resumen, en la Parte I de esta tesis se introduce brevemente un contexto actualizado del conocimiento general de estas estrellas, partiendo de un recuento de los principales hechos históricos relacionados con su estudio, se presenta la fenomenología asociada y sus características principales para luego pasar a la descripción de las herramientas utilizadas para su estudio y modelado. Finalmente se brinda una introducción al análisis de datos en la banda de los rayos X y los instrumentos que usamos en esta tesis. En el Capítulo 1 se presentan además las motivaciones, resultados y preguntas que dieron lugar a toda esta investigación. En los capítulos posteriores se presentan los trabajos científicos específicos realizados que conforman esta tesis doctoral.

En la Parte II se presentan dos modelos teóricos desarrollados para su estudio y los resultados obtenidos, por un lado, para la evolución del campo magnético en NSs que sufren un proceso de acreción de materia en un HMXB y, por otro, para explicar el frenado repentino de un magnetar a partir de la evolución de su campo magnético interior que podría tener efectos sobre la estructura global de la corteza de la estrella. En la Parte III presentamos los resultados alcanzados en dos estudios realizados a partir del análisis de datos obtenidos en la banda de los rayos X por los satélites RXTE, Chandra y XMM-Newton. Por un lado, asociados al estudio del enfriamiento de la atmósfera de la NS en el LMXB 4U 1820-30 y por otro a la búsqueda de un remanente compacto en la emisión extendida asociada al SNR G290.1-0.8. Finalmente, la Parte IV involucra el desarrollo teórico de una línea de investigación reciente en la que las NSs juegan un papel fundamental, en el que se pone a prueba teorías alternativas a la GR de Einstein en el régimen de campo gravitacional fuerte o de gran curvatura. En particular presentamos aportes realizados al estudio de la estructura de NSs construidas en gravedad $f(R)$ cuadrática, tanto en el formalismo métrico como en el de Palatini. De esta manera, se concentran aquí los aportes realizados al estudio de NSs en escenarios astrofísicos diversos y utilizando diferentes enfoques metodológicos ya sea a partir del desarrollo de modelos analíticos, la programación de códigos numéricos semianalíticos, o el análisis de datos de rayos X asociados a NSs obtenidos por distintos observatorios orbitales. Los aportes realizados en esta tesis abren, a su vez nuevas líneas de investigación, enmarcadas dentro de la astrofísica de altas energías. 
A continuación se resumen las principales conclusiones y aportes alcanzados en conjunto con posibles líneas de trabajo a explorar, que permitirán ampliar y profundizar los estudios llevados adelante en esta tesis.

\section{Parte II}

Los modelos teóricos presentados en la Parte II de esta tesis muestran principalmente que el campo magnético de estas estrellas juega un rol fundamental en su evolución y puede ser fuente de fenómenos particulares observados tanto en NSs aisladas con campos magnéticos muy intensos, como los magnetares, como en el caso de NSs pertenecientes a sistemas binarios.

En el Capítulo 2 se introduce el desarrollo de un modelo numérico para la evolución del campo magnético de una NS que acreta materia proveniente del viento generado por una estrella de gran masa en un sistema binario. El desarrollo del modelo fue motivado por la necesidad de estudiar condiciones necesarias para la formación de jets relativistas en este tipo de sistemas binarios, producto del decaimiento del campo magnético de la NS que podría ser acelerado por el proceso de acreción.

El modelo numérico desarrollado incorpora el estado del arte en la microfísica de la estrella y hace uso de perfiles de evolución térmica obtenidos de forma consistente con nuestro modelo (Aguilera et al., 2008). La ecuación de inducción que resolvemos para analizar la evolución del campo magnético en la corteza, considera la difusión óhmica de las corrientes eléctricas y la advección de las líneas de campo magnético, arrastradas por el flujo de materia acretada sobre su superficie. La mayor limitación del modelo radica en que se considera únicamente acreción esférica, y no acreción en forma de columnas a través de los polos magnéticos, y su posterior redistribución en toda la superficie de la estrella. A pesar de eso, a modo cualitativo, resulta esperable que fenómenos como el enterramiento del campo magnético de la estrella, y su difusión, responsables del decaimiento acelerado, jueguen un rol importante si el área sometida a la acreción inicial es menor, ya que la tasa de acreción local de materia resultaría amplificada por el mismo factor. Así, la posibilidad de que jets relativistas puedan ser emitidos en este contexto, merece ser estudiada en mayor detalle.

Nuestros resultados cuantitativos muestran una dependencia fundamental entre la tasa de acreción y la escala temporal de decaimiento del campo magnético. Además, otros parámetros del modelo, como el contenido de impurezas de la corteza y el estado de superfluidez y la masa de la NS, que modifican su perfil de enfriamiento, son determinantes en un segundo plano. De esta manera, en el caso que una fracción importante del viento emitido por la estrella compañera pudiera ser acretada por la NS, su campo magnético podría decaer desde un valor inicial típico de un pulsar $\left(B \sim 10^{12} \mathrm{G}\right)$ hasta valores que admiten la formación de jets relativistas $\left(B \lesssim 10^{8} \mathrm{G}\right.$, Massi \& Kaufman Bernadó, 2008) en la escala temporal impuesta por la evolución de la estrella compañera de gran masa, que es de unos $\sim 10^{7}$ años.

Los resultados expuestos en esta parte de la tesis tienen impacto sobre modelos de emisión de altas energías en SFXTs que involucran NSs en HMXBs, pudiendo convertir a estos sistemas en una nueva clase de fuentes de rayos $\gamma$ de $\mathrm{MeV}$ en la Galaxia, como fuera sugerido por Sguera et al. 
(2009) para AX J1841.0-0536. Además, los resultados son aplicables a binarias de rayos $\gamma$ tales como LS I +61 303 o LS 5039, objetos paradigmáticos en lo que respecta a emisión no térmica de muy altas energías. Simulaciones numéricas realizadas por Owocki et al. (2011), revelan tasas de acreción de materia del orden de $\sim 10^{-10} \mathrm{M}_{\odot}$ año ${ }^{-1}$, como las deducidas a partir de nuestro modelo.

El seguimiento de estas fuentes usando observaciones en la banda de los rayos $\gamma$ de los satélites Fermi y AGILE, así como observaciones dedicadas a más altas energías usando MAGIC II y HESS II son importantes para establecer cotas al nivel de radiación no térmica durante las fulguraciones de rayos X, posibilitando la determinación de la energía máxima de corte de las partículas relativistas. La combinación con observaciones interferométricas en radio podría ayudar a confirmar o descartar la formación de jets. Del mismo modo, la detección de un jet transitorio en un HMXB que albergue una NS, brindaría evidencia indirecta de nuestras predicciones para el decaimiento del campo magnético.

Los primeros modelos que incorporan la posibilidad de estudiar acreción columnar, así como el acoplamiento de la evolución del campo magnético con la evolución térmica de la estrella fueron realizados por Viganò et al. (2012, 2013). Su aplicación al problema observacional que motiva nuestro trabajo es un objetivo que también merece ser estudiado con más detalle en el futuro.

Dentro del contexto del estudio de la evolución a largo plazo del campo magnético de NSs, en el Capítulo 3 presentamos un modelo sencillo que permite explicar la disminución repentina de la velocidad de rotación observada en el magnetar AXP 1E 2259+586, fenómeno conocido como anti-glitch. Nuestro modelo se basa en la hipótesis de que el decaimiento de la componente toroidal de su campo magnético, sería capaz de desestabilizar una configuración originalmente estable y alargada, llevándola a una nueva forma más esférica que resulte mecánicamente estable bajo la configuración de campo magnético actual. Así, el frenado observado en (Archibald et al., 2013), aparecería naturalmente como consecuencia de la conservación del momento angular. La liberación de energía estimada en $\sim 10^{42}$ erg resulta estar en completo acuerdo con la emisión detectada por los observatorios Fermi y Swift en la época en que ocurrió dicho evento.

La principal predicción contrastable de nuestro modelo surge del hecho que un frenado como el detectado por Archibald et al. (2013) en AXP 1E 2259+586, sólo podría ser alcanzado si la intensidad promedio inicial del campo magnético toroidal interno de la NS es varias veces superior a $10^{14} \mathrm{G}$, como en el caso de los magnetares. Consecuentemente, este valor crítico impide el desarrollo de un anti-glitch de tal magnitud en los pulsares propulsados por rotación. Esta predicción explica de manera natural la razón por la cual este evento es el primero en su tipo, dado que si bien la extensa población de pulsares ordinarios ha sido largamente monitoreada durante $\sim 50$ años, sólo se conocen unos 10 a 20 magnetares, que han sido monitoreados durante los últimos 20 años.

Con el objetivo de realizar las primeras estimaciones para poner a prueba la idea desarrollada manteniendo la analiticidad de nuestro modelo, consideramos únicamente una estrella de densidad uniforme cuya estructura es afectada por un campo magnético netamente toroidal. El desarrollo de un modelo más detallado que considere, por ejemplo, a una NS compuesta por una corteza sólida rodeando un núcleo líquido (como el propuesto por Franco et al., 2000, para estudiar terremotos 
estelares en pulsares alimentados por rotación) es uno de los principales objetivos a desarrollar a futuro. En este sentido, Frieben \& Rezzolla (2012) han desarrollado modelos relativistas de NSs elipsoidales considerando EoSs realistas, encontrando elipticidades compatibles con las obtenidas en esta tesis. La adaptación de estos modelos al estudio de anti-glitches en este y otros magnetares, resulta una fuente de información vital acerca de las propiedades de la corteza que merece ser estudiada en mayor profundidad.

Nuestro modelo simple fue ampliado recientemente por Mastrano et al. (2015), incorporando el decaimiento de una configuración de campo magnético con más órdenes en el desarrollo multipolar. En su trabajo, los autores calculan el cambio en la elipticidad, $\epsilon$, debido a un campo entrelazado dipolar-toroidal, y para una configuración dipolar con un toro cuadrupolar entrelazado. De esta manera muestran que la omisión de la contribución de la componente poloidal del campo magnético en la estructura de la estrella da como resultado una sobreestimación del alargamiento de la estrella. Cabe aclarar que en nuestro caso, esta componente fue ignorada intencionalmente para mantener la analiticidad del modelo. Incluyendo el campo dipolar en la configuración, o incorporando un campo dipolar-cuadrupolar trenzado con el campo toroidal, Mastrano et al. (2015) encuentran que únicamente objetos con $B_{d} \gtrsim 10^{13} \mathrm{G}$ pueden producir el cambio en la frecuencia observado, $\Delta v / v$, confirmando una de las conclusiones principales de nuestro modelo. En este sentido, más allá de las similitudes observacionales, la naturaleza del frenado o anti-glitch experimentado por el magnetar AXP 1E 2259+586 tendría un origen físico completamente diferente a las anomalías o glitches de los pulsares, o incluso de los propios magnetares. De esta manera, los autores arriban a conclusiones cuantitativamente diferentes, pero cualitativamente sus resultados son idénticos a los nuestros (ver Figura 3 de Mastrano et al., 2015).

Por otra parte, Lander et al. (2015) estudiaron las erupciones de los magnetares, imponiendo una cota máxima de $4 \times 10^{46}$ erg para la energía liberada, producto de una fractura en la corteza, independiente de la intensidad del campo magnético. Esto significa que las erupciones más energéticas de estas fuentes podrían ser explicadas por este tipo de "terremotos" estelares. Si bien la posibilidad de diferenciar las contribuciones energéticas de la propia corteza y del campo magnético es difícil en la práctica, en el caso que una erupción tal sea acompañada por un frenado repentino, entonces una fracción significativa de la energía liberada debería originarse en la reconfiguración del campo magnético.

Es importante mencionar que el frenado observado en AXP 1E 2259+586 ha sido interpretado también en otros escenarios. Por ejemplo, Katz (2013) propuso que el fenómeno es producto de la acreción de material de la supernova que originó la NS, y Huang \& Geng (2014) sugirieron la colisión con un asteroide de $\sim 10^{18} \mathrm{~kg}$. Por otro lado, Kantor \& Gusakov (2014) intentaron explicar el frenado dentro del escenario estándar de las anomalías o glitches, producto de un retraso entre la velocidad de rotación del superfluido del núcleo de la estrella y su corteza que se reconectan abruptamente. En todos estos trabajos el frenado es una consecuencia, pero en ningún caso se modela el mecanismo que lo dispara ni la escala temporal asociada. Así como en nuestro trabajo proponemos el decaimiento del campo magnético interno como la fuente del frenado observado, Link (2014) y Lyutikov (2015) sugieren, en cambio, que las erupciones y estallidos de 
los magnetares son producto de la relajación de la configuración externa del campo magnético.

La simulación del decaimiento del campo magnético que produce el quiebre de la corteza y el cálculo de las escalas temporales involucradas conforman un desafío interesante a futuro. Entendemos que el estudio detallado de la fenomenología de los anti-glitches podría aportar conocimiento acerca de las propiedades mecánicas de la materia nuclear a alta densidad que compone la corteza de las NSs altamente magnetizadas. Al mismo tiempo, el monitoreo preciso y continuo de estas fuentes resulta indispensable para establecer restricciones precisas a estos modelos. Finalmente, cabe mencionar que en el escenario propuesto, cabría esperar la emisión de ondas gravitacionales de alta frecuencia, tanto como producto del cambio en la forma global de la estrella durante el frenado, como por oscilaciones posteriores, que podrían ser detectadas por el experimento LIGO$^{1}$.

\section{Parte III}

En cuanto a la Parte III, en el Capítulo 4 se desarrollan los resultados asociados a la búsqueda de un remanente compacto, posiblemente un CCO, en el SNR G290.1-0.8, que incluye el análisis detallado de la emisión extendida utilizando observaciones de los satélites XMM-Newton y Chandra. El análisis de la región central del remanente reveló tres fuentes puntuales en su interior. Dos de ellas son fuentes caracterizadas por emisión de rayos $\mathrm{X}$ predominantemente duros, como se deduce del análisis morfológico de los colores. Esto sugiere un origen extragaláctico asociado a núcleos activos de galaxias de fondo. La tercera, en cambio, próxima al centro geométrico proyectado del SNR, es dominada por emisión de rayos X blandos, y se encuentra inmersa en una estructura de emisión difusa. La búsqueda de pulsaciones asociadas a esta fuente no arrojó ningún período asociado. Tampoco hallamos contrapartidas en otras longitudes de onda a partir de los datos disponibles en la literatura. Estos resultados compatibilizan a la fuente con las características típicas de un CCO de campo magnético débil (Halpern \& Gotthelf, 2010). Sin embargo, con los datos disponibles no es factible descartar la posibilidad que la emisión sea originada en un nodo del plasma asociado al remanente. Observaciones profundas con Chandra podrían ayudar a revelar más detalles de la emisión de rayos X y discernir la naturaleza de esta fuente.

El mapa de energía media de los fotones detectados indica que las condiciones físicas del plasma asociado al SNR son fuertemente inhomogéneas. El análisis espectral para las cinco regiones estudiadas lo confirma. Del análisis espectral se deduce que mientras que el plasma de las regiones Central, Noreste y Sudoeste ha alcanzado el equilibrio de ionización, en las zonas más expandidas, en la dirección Noroeste y Sudeste, el plasma permanece fuera del equilibrio. Además, las temperaturas de las primeras regiones son significativamente inferiores a las obtenidas en las regiones ubicadas a lo largo del eje de simetría Noroeste-Sudeste. Por otro lado, si bien las abundancias químicas son diferentes, en todos los casos corresponden a material eyectado por la explosión de supernova y no al medio interestelar preexistente. La baja abundancia de $\mathrm{Fe}(\lesssim 0.1)$ y los cocientes de abundancias de $\mathrm{Mg} / \mathrm{Si} \sim 0.5, \mathrm{~S} / \mathrm{Si} \sim 0.8$ y Fe/Si 0.05 (en la región central) son consistentes una

${ }^{1}$ http: //www . ligo.org/ 
explosión de supernova debida al colapso de una estrella masiva de 25-30 $\mathrm{M}_{\odot}$ (Woosley \& Weaver, 1995) y fuertemente incompatibles con cualquier escenario degenerado (Troja et al., 2008). En tal escenario, un fuerte viento bipolar producido por la estrella progenitora podría haber formado la cavidad alargada en la que posteriormente se expandió el SNR hasta su forma actual. En el trabajo clásico de Lamers \& Cassinelli (1999), se muestra que, a través de un viento asimétrico, las estrellas de gran masa pueden transferir $\sim 10^{51}$ erg de energía mecánica a su entorno.

A partir de observaciones profundas realizadas con Chandra, Pavan et al. (2014) sugirieron que una fuente puntual asociada a emisión extendida predominantemente dura, ubicada a unos 7' al sudoeste de G290.1-0.8, podría contener un remanente compacto producto de la explosión de supernova. La alineación de la emisión extendida con la dirección del SNR, junto a la distancia derivada por la columna de absorción, apoyan esta posibilidad, requiriendo entonces que el pulsar esté atravesando el medio interestelar con una velocidad de entre 1100 y $2200 \mathrm{~km} \mathrm{~s}^{-1}$. De ser así, esta fuente podría convertirse en el pulsar más rápido de la Galaxia. Recientemente, Halpern et al. (2014) observaron esta fuente utilizando el modo de lectura rápida timing de XMM-Newton en dos oportunidades, separadas por 322 días, descubriendo emisión periódica de $62.8 \mathrm{~ms}$ y derivada $8.56 \pm 0.51 \times 10^{-15}$, que permitió confirmar que la fuente es un pulsar alimentado por rotación, aunque su luminosidad resultó ser un orden de magnitud menor a la predicha. Con una edad característica de 116 mil años, la probabilidad de asociación con G290.1-0.8 resulta muy alta. En el futuro, la determinación del movimiento propio de la fuente puntual podría confirmar o rechazar la asociación física de ambas fuentes.

En ese caso, la búsqueda del objeto compacto en el interior de G290.1-0.8 no habría resultado satisfactoria. Cabe destacar que en otros trabajos similares realizados por nuestro grupo (Combi et al., 2010; Sánchez-Ayaso et al., 2012), en cambio, las observaciones revelaron dos candidatos a CCOs, lo que motiva la realización de este tipo de investigación en trabajos futuros. Si bien, la detección no está garantizada, el propio estudio detallado de la emisión extendida en SNRs es importante en sí mismo. La determinación de las condiciones físicas de los plasmas emisores, así como la distribución de los elementos químicos detectados en ellos a partir de la espectroscopía, en conjunto con el análisis detallado de la morfología en rayos X e infrarrojo permiten determinar la conexión entre los remanentes y el tipo de explosión de supernova que los originó. La complementación de estos estudios con el análisis en otras longitudes de onda como el continuo de radio, emisión de líneas de $\mathrm{H}, \mathrm{CO}$ y emisión extendida en rayos $\gamma$, permite realizar aportes vitales para comprender en detalle los escenarios astrofísicos involucrados.

En un trabajo reciente, usando los datos recolectados por el satélite Fermi en rayos $\gamma$, Auchettl et al. (2015) reportan la detección de emisión extendida asociada a G290.1-0.8. La detección es consistente con una fuente localizada a lo largo o dentro del borde oeste del remanente. El espectro de la emisión detectada es consistente con un modelo hadrónico, mientras que un escenario leptónico resulta energéticamente poco probable, lo que favorece un escenario de interacción con una región de $\mathrm{HI}$ o $\mathrm{CO}$ en la dirección norte y sudoeste en acuerdo con nuestro análisis.

En el Capítulo 5, presentamos el primer análisis espectral sistemático de las 16 erupciones termonucleares de rayos X detectadas con el satélite RXTE en el LMXB 4U 1820-30 donde mostramos que todas las erupciones son del tipo-I y presentan expansión fotosférica (tipo PRE). 
Todos los parámetros que caracterizan a la fase de enfriamiento como la temperatura y el flujo bolométrico en el punto de contacto, la fluencia y la escala de tiempo de enfriamiento o su duración, son consistentes entre sí considerando todas las erupciones detectadas. Esto nos permitió combinar las fases de enfriamiento de las 16 erupciones para mejorar la estadística. De esta manera, encontramos que el ajuste espectral de las curvas de enfriamiento de todas las erupciones, encontramos que ésta no sigue la relación $F \propto\left(k T_{\mathrm{bb}}\right)^{4}$, como sería esperable en el caso de un cuerpo negro que se enfría con área de emisión constante. En cambio, el flujo bolométrico y la temperatura se relacionan por una ley de potencias quebrada de índices $2.0 \pm 0.3$ en la primera etapa y $5.72 \pm 0.06$ hacia el final.

La consecuencia inmediata de este resultado es que el apartamiento de la relación $F \propto\left(k T_{\mathrm{bb}}\right)^{4}$ no permite usar la fase de enfriamiento de las erupciones de rayos X del sistema 4U 1820-30 para determinar la masa y el radio de su NS con la precisión anunciada por Güver et al. (2010). Considerando la totalidad de las erupciones observadas, no existe siquiera una porción del diagrama en la que la relación $F$ vs. $k T_{\text {bb }}$ pueda ser ajustada por una ley con potencia con índice igual a 4. La tendencia que encontramos en la fase de enfriamiento es similar a la de las erupciones noPRE de 4U 1636-53 para emisión persistente dura (Zhang et al., 2011). En ambos casos, el flujo bolométrico decae más rápido que lo esperado para un cuerpo negro de radio constante. Por otro lado, del análisis del superburst observado por RXTE en 4U 1820-30, Miller (2013) concluyó que no puede garantizarse que la estrella haya emitido a través de la totalidad de su superficie en las más de tres horas que duró la erupción (Strohmayer \& Brown, 2002). Esto impide su comparación con las 16 erupciones de rayos $\mathrm{X}$ de tipo-I estudiadas por nosotros, donde el flujo de fotones es mucho menor y por tanto insuficiente.

El apartamiento de la relación $F$ vs. $k T_{\mathrm{bb}}^{4}$ podría darse al menos por tres razones diferentes -no mutuamente excluyentes-: cambios en el área de emisión de la estrella $\left(R_{\mathrm{bb}}\right)$, modificaciones del factor de corrección de color $\left(f_{c}\right)$ o la presencia de una fuente de calor remanente. Esta última posibilidad, podría explicar además el índice de ley de potencia más grande que cuatro obtenido en la relación global entre el flujo y la temperatura del cuerpo negro (Cumming, 2003). Variaciones en el área de emisión de la estrella podrían ser evidenciadas por la presencia de oscilaciones en su emisión (ver, por ejemplo, Strohmayer \& Bildsten, 2006). Sin embargo, estas oscilaciones no fueron detectadas en las erupciones de 4U 1820-30 (Galloway et al., 2008; Watts, 2012). Por el contrario, si el área de emisión es constante, las variaciones en $R_{\mathrm{bb}}$ podrían ser explicadas por cambios en $f_{\mathrm{c}}$ Recientemente, Zhang et al. (2016) analizaron las 121 erupciones de rayos X tipo-I detectadas por RXTE en el LMXB 4U 1728-34, y encontraron que la duración de la subida en el brillo y la convexidad de las curvas de luz asociadas son diferentes para erupciones con o sin oscilaciones. En la primera parte de la fase de enfriamiento de las erupciones, el espectro de energía es diferente en erupciones con o sin oscilaciones. En esta fuente, sólo las erupciones que no presentan oscilaciones pueden ajustarse usando modelos de atmósferas de NSs. Por el contrario, el radio del cuerpo negro ajustado en los espectros permanece constante (y por lo tanto, $F \propto T^{4}$ ) por al menos $2 \mathrm{~s}$, sólo en las erupciones que presentan oscilaciones.

La disminución del factor de corrección de color en los momentos posteriores al punto de contacto de las erupciones PRE son predichas por los modelos de atmósferas de NSs (Suleimanov et 
al., 2011). Este efecto podría dar lugar a la ley de potencias quebrada como la que mejor ajusta al enfriamiento de $4 \mathrm{U}$ 1820-30, siendo un patrón que se repite en otros sistemas similares. Kajava et al. (2014) analizaron las 246 erupciones de rayos X observadas por RXTE en 11 LMXBs enfocándose en la relación entre las propiedades espectrales de la emisión persistente y la evolución temporal de la normalización del cuerpo negro durante la fase de enfriamiento de las erupciones. De su análisis los autores deducen que las predicciones de los modelos de enfriamiento podrían ajustar únicamente para una gran parte de las erupciones que ocurren cuando la emisión persistente presenta espectros duros y bajas luminosidades en los diagrama CD y HID. En cambio, muestran que esto rara vez ocurre cuando las erupciones están asociadas a emisión persistente en el estado blando y de alta luminosidad. Kajava et al. (2014) asocian este comportamiento al flujo de material acretado, que en el caso de estar activo podría influir en el proceso de enfriamiento posterior a las erupciones. En este marco, si bien las erupciones detectadas en 4U 1820-30, corresponden a emisión persistente en el estado duro o en la transición del estado duro al estado blando, a relativamente baja luminosidad, su curva de enfriamiento no puede ajustarse por una única ley de potencias, $F \propto\left(k T_{\mathrm{bb}}\right)^{4}$, y tampoco son ajustadas en su totalidad por los modelos de atmósfera actuales, realizados por Suleimanov et al. (2011).

Las características de las observaciones disponibles en la actualidad, provenientes de las erupciones detectadas esencialmente por el satélite $R X T E$ distan de poder brindar candidatos que permitan determinar simultáneamente la masa y el radio de NSs con suficiente precisión en estos sistemas. Es aquí donde la próxima generación de observatorios de rayos $\mathrm{X}$ conformada por los experimentos NICER y $L O F T^{3}$ jugarán un papel fundamental, ya que el área efectiva y la resolución temporal con que estos instrumentos están diseñados posibilitará el alcance de grandes avances en el camino hacia la determinación precisa de los radios y masas de NSs en LMXBs, permitiendo a su vez allanar el camino hacia la determinación de la EoS de la materia a alta densidad.

Los resultados aquí presentados, así como los recientemente publicados por Kajava et al. (2014) y Ozel et al. (2015), motivan la extensión del análisis sistemático realizado para las 16 erupciones detectadas en 4U 1820-30 a otras fuentes similares. En particular, resulta de interés avanzar sobre las erupciones asociadas a pulsares de rayos $\mathrm{X}$ donde el campo magnético, responsable de la formación de hotspots rotantes que dan origen a los pulsos, podría estar involucrado. Las herramientas de análisis de datos para la detección de erupciones, extracción de espectros y su ajuste automático, que desarrollamos y mejoramos a lo largo de este proyecto, nos permitirán realizar esta nueva extensión sin grandes impedimentos.

\section{Parte IV}

En la Parte IV presentamos nuestros aportes a las investigaciones de NSs en teorías alternativas a GR. El estudio de estrellas compactas en teorías de gravedad alternativas tiene una larga historia. Si bien las configuraciones de equilibrio de NSs y sus correspondientes diagramas

\footnotetext{
${ }^{2}$ https://heasarc.gsfc.nasa.gov/docs/nicer/

${ }^{3}$ http://sci.esa.int/loft/;http://www.isdc.unige.ch/loft/
} 
masa-radio asociados han sido largamente explorados en muchas clases diferentes de estas teorías, su estabilidad y propiedades dinámicas continúan siendo una gran incógnita. Así mismo, la existencia de regiones como las encontradas en el caso de teorías de gravedad cuadrática tanto en el formalismo métrico como el de Palatini, donde la masa encerrada decrece con la coordenada radial, podrían dar lugar a inestabilidades que pondrían cotas superiores muy restrictivas al parámetro libre de la teoría, pudiendo volverlas inviables.

Por otra parte, las grandes incertezas que existen en el conocimiento de la microfísica de la materia a alta densidad, expresado a través de la EoS, se transforma en incertezas en la relación masa-radio de estas estrellas. Esto, en conjunto con las incertezas subyacentes en la determinación precisa del radio de las NSs a través de su observación, dificultan la prueba de teorías alternativas a la gravedad en estos casos. Por ejemplo, para una NS típica con una masa de $1.4 \mathrm{M}_{\odot}$, las diversas EoSs compatibles con el conocimiento actual de la física nuclear predicen radios en el rango de 6 a 16 km (Steiner et al., 2013). La degeneración entre los efectos introducidos por la extensión de teorías alternativas a GR y las incertezas en la EoS de las NSs constituye una limitación intrínseca a la posibilidad de realizar pruebas precisas de la gravedad a partir de observaciones de NSs. Las incertezas en la EoS se traducen en incertezas en los observables macroscópicos, tales como las masas, radios y frecuencias de oscilación, que resultan en muchos casos, incluso mayores que las posibles desviaciones de GR, como en los casos que se analizan en esta tesis.

Como las teorías gravitatorias con campos auxiliares básicamente no poseen restricciones surgidas del límite de campo débil, y sólo se vuelven notorias cuando se acoplan fuertemente con la materia, las NSs representan laboratorios ideales para acotarlas. Lamentablemente, en la actualidad con la precisión con la que contamos a partir de las observaciones, las teorías aquí analizadas resultan totalmente degeneradas con las incertezas provenientes de la propia EoS. La razón yace en que esta teoría no contiene campos dinámicos adicionales, por lo que la modificación introducida en el lado derecho de las ecuaciones de campo funciona como un fluido efectivo que aporta a la EoS. Esta ambigüedad intrínseca entre las modificaciones en el acoplamiento de la gravedad con la materia y las variaciones en la EoS, hacen poco viable la restricción de estas teorías con la información obtenida a través de las observaciones disponibles en la actualidad.

Una posibilidad interesante surge de ampliar el estudio de estas configuraciones agregando una dimensión espacial para pasar a analizar modelos de NSs axisimétricas, que incorporen por ejemplo los efectos de la rotación, o campos magnéticos intensos. Los modelos de estrellas rotantes son difíciles de construir. Sin embargo, como las NSs más viejas rotan con velocidades relativamente bajas, a menos que sean aceleradas por la acreción de material de una estrella compañera por medio de un disco de acreción, pueden realizarse cálculos perturbativos usando expansiones compatibles con rotación lenta que pueden ser de interés para casos astrofísicos. El formalismo para construir estrellas rotantes fue desarrollado en un trabajo fundacional por Hartle \& Thorne (1968) y ha sido expandido a cuarto orden en la rotación (Yagi et al., 2014). En dos trabajos recientes, Staykov et al. (2014) y Yazadjiev et al. (2015) realizaron los primeros cálculos de NSs en teorías escalar-tensoriales matemáticamente equivalentes a las teorías $f(R)$ incorporando los efectos introducidos por la rotación en las configuraciones. En el primer trabajo se obtienen soluciones bajo una aproximación lenta para la métrica mientras que en el segundo se tratan rotaciones rá- 
pidas. Sus primeros resultados muestran que las desviaciones de GR son amplificadas, pudiendo dar lugar a efectos potencialmente observables.

Como discutimos en el Capítulo 1, la propia existencia de estrellas compactas en teorías de gravedad $f(R)$ es materia de discusión en la actualidad. En particular ciertos comportamientos propios de las teorías de gravedad $f(R)$ bajo el formalismo de Palatini representan conflictos con el modelo estándar y los resultados provenientes de la cosmología Olmo (2011), lo que impone serias dudas acerca de la viabilidad de este tipo de teorías. En cualquier caso, resulta fundamental ponerlas a prueba en el régimen de campo fuerte, para obtener mayor información acerca de sus capacidades, que puedan motivar su estudio o directamente ser descartadas.

En muchas extensiones de GR, el principio de equivalencia es violado debido a la presencia de campos adicionales. Objetos autogravitantes, como el caso de las NSs, resultan sensibles a las variaciones en el campo gravitacional introducidas por estos mismos campos. Cuando estos objetos se mueven en regiones del espacio tiempo donde el campo gravitacional no es constante, su energía gravitacional interna, y por tanto su masa total puede verse modificada. Así, este efecto constituye una nueva prueba a las teorías alternativas donde, por ejemplo, dos NSs interaccionen fuertemente en un sistema binario. Este tipo de pruebas es un ejemplo de desarrollo a futuro, necesario para la mayoría de estas teorías (Berti et al., 2015).

Otro camino a explorar a futuro lo constituye el análisis de la estabilidad de las soluciones estáticas encontradas para las diferentes EoSs en estas teorías. En este sentido, resulta interesante investigar las consecuencias físicas que tienen las regiones donde $\mathrm{d} m / \mathrm{d} r<0$ sobre la estructura de la estrella. Para ello es posible proceder como en GR, proponiendo soluciones con simetría esférica pero dependientes del tiempo y estudiar perturbaciones radiales a las soluciones entorno a las soluciones estáticas obtenidas en esta tesis.

Por otro lado, una rama que no ha sido encarada por ningún grupo en la actualidad la constituye el estudio de las propiedades del transporte de calor en estas teorías. En particular, una línea de investigación concreta que podría encararse en el futuro, y que requiere de desarrollos analíticos y numéricos no tan profundos, es la evolución térmica de estas configuraciones. Esto permitiría realizar contrastaciones directas con, por ejemplo, las observaciones del enfriamiento de NSs que han sufrido largos períodos de acreción en LMXBs. Para ello, se podría proceder incorporando a los códigos de evolución térmica disponibles la información de los coeficientes de la métrica de las configuraciones estáticas aquí presentadas, y que entran en la ecuación de difusión relativista. De esta manera se podría estudiar cómo las variaciones de la métrica en la región de transición entre la corteza interna y la externa, donde justamente $\mathrm{d} m / \mathrm{d} r<0$, influyen sobre las curvas de enfriamiento de estas estrellas, largamente estudiadas (Turlione et al., 2015).

En el futuro, la compacticidad de las NSs podría ser determinada a partir de la medición precisa de sus radios y masas, que se espera alcanzar con la próxima generación de observatorios de rayos $\mathrm{X}$, como ya mencionamos. Incorporando soluciones que den cuenta de los efectos producidos por la rotación y algoritmos capaces de trazar las geodésicas nulas entorno a estas estrellas, se podrán calcular, por ejemplo, los perfiles de las hotspots rotantes en la superficie de NSs en diferentes teorías de gravedad, lo que permitirá ajustar simultáneamente los parámetros macroscópicos de las estrellas junto con las constantes de acoplamiento de estas teorías. 


\section{Bibliografía}

Aguilera, D. N., Pons, J. A., \& Miralles, J. A. 2008, A\&A, 486, 255 10, 54, 55, 68, 69, 146 Aguilera, D. N., Cirigliano, V., Pons, J. A., Reddy, S., \& Sharma, R. 2009, PRL, 102, 091101135

Akgün, T., Reisenegger, A., Mastrano, A., \& Marchant, P. 2013, MNRAS, 433, 244527

Amanullah, R., Lidman, C., Rubin, D., et al. 2010, ApJ, 716, 712118

Anders, E., \& Grevesse, N. 1989, GCA, 53, 19787

Anderson, P. W., \& Itoh, N. 1975, Nature, 256, 2512

Antoniadis, J., Freire, P. C. C.,Wex, N., et al. 2013, Science, 340, 448 22, 137

Arapoğlu, S., Deliduman, C., \& Yavuz Ekşi, K. 2011, JCAP, 7, 20 118, 120, 121, 122, 123, 128

Archibald, R. F., Kaspi, V. M., Ng, C.-Y., et al. 2013, Nature, 497, 591 12, 28, 72, 78, 147

Arnaud K. A., 1996, ASPC, 101, 17 43, 89, 101

Arnaud, K., Smith, R., Siemiginowska, A., et al. 2011, Handbook of X-ray Astronomy, Cambridge University Press. 42

Auchettl, K., Slane, P., Castro, D., Foster, A. R., \& Smith, R. K. 2015, ApJ, 810, 43150

Baade W., \& Zwicky, F. 1934, Proc. Nat. Acad. Sci. 20, 2543

Babichev, E., \& Langlois, D. 2010, PRD, 81(12), 124051118

Baiko, D. A., Kaminker, A. D., Potekhin, A. Y., Yakovlev, D. G. 1998, PRL, 81, 555632

Baiko, D. A., Haensel, P., \& Yakovlev, D. G. 2001, A\&A, 374, 15132

Balucinska-Church, M., \& McCammon, D. 1992, ApJ, 400, 69990

Bamba, A., Yokogawa, J., Ueno, M., Koyama, K., \& Yamauchi, S. 2001, PASJ, 53, 117965

Barausse, E., Sotiriou, T. P., \& Miller, J. C. 2008a, C\&QG, 25(6), 062001 132, 133, 134, 135, 137, 142

Barausse, E., Sotiriou, T. P., \& Miller, J. C. 2008b, en EAS Publications Series vol. 30, ed. por Oscoz, Mediavilla, Serra-Ricart 132

Barausse, E., Sotiriou, T. P., \& Miller, J. C. 2008c, C\&QG, 25(10), 105008132

Barragán, C. Olmo, G. J., \& Sanchis-Alepuz, H. 2009, PRD, 80(2), 024016135

Basinska, E. M., Lewin, W. H. G., Sztajno, M., Cominsky, L. R., \& Marshall, F. J. 1984, ApJ, 281, 337 100

Bhattacharya, D. 2002, JAA, 23, 67-72 10

Baym, G., Pethick, C. \& Sutherland, P. 1971, ApJ, 170, 29922

Baym, G., \& Pines, D. 1971, Annals of Physics, 66, 816 12, 75, 77

Bejger, M., Haensel, P., \& Zdunik, J. L. 2005, MNRAS, 359, 699140

Berti, E., Barausse, E., Cardoso, V., et al. 2015, C\&QG, 32, 243001 28, 31, 154

Bildsten, L. 1995, ApJ, 438, 852100

Bildsten, L. 1998, NATO Advanced Science Institutes (ASI) Series C, 515, 41917

Bisnovatyi-Kogan, G. S., \& Komberg, B. V. 1974, Sov. Ast., 18, 21753

Blondin, J. M., Lundqvist, P., \& Chevalier, R. A. 1996, ApJ, 472, 25784

Bocchino, F., Miceli, M., \& Troja, E. 2009, A\&A, 498, 13995 
Bolejko, K., Célérier, M.-N., \& Krasiński, A. 2011, C\&QG, 28(16), 164002118

Bosch-Ramon, V., Paredes, J. M., Romero, G. E., \& Rib, M. 2006, A\&A, 459, L25 67

Boynton, P. E., Groth, E. J., III, Partridge, R. B., \& Wilkinson, D. T. 1969, IAU Circular, 217912

Bozzo, E., Falanga, M., \& Stella, L. 2008, ApJ, 683, 1031 52, 64

Bozzo, E., Pavan, L., Ferrigno, C., et al. 2012, A\&A, 544, A118 66

Bradt, H. V., Rothschild, R. E., \& Swank, J. H. 1993, A\&AS, 97, 35538

Braithwaite, J. 2009, MNRAS, 397, 763 27, 73

Braun, R., \& Strom, R. G. 1986, A\&A, 164, 20884

Brown, E. 2000, ApJ, 531, 988B 24

Bulgarelli, A., Gianotti, F., Trifoglio, M., et al. 2009, ATel, 2017, 166

Burrows, A., \& Lattimer, J. M. 1984, ApJ, 285, 294B 22

Capozziello, S. \& Faraoni, V. 2011, Beyond Einstein Gravity. A survey of Gravitational Theories for Cosmology and Astrophysics, Springer 29, 30, 118, 120, 131, 132

Cardall, C. Y., Prakash, M., \& Lattimer, J. M. 2001, ApJ, 554, 32227

Carter, J. A., \& Read, A. M. 2007, A\&A, 464, 115589

Cash, W. 1979, ApJ, 228, 939103

Chadwick, J. 1932, Nature, 129, 3123

Chamel, N., \& Haensel, P. 2008, Living Reviews in Relativity, 11, 10 22, 72

Chen, A., Vercellone, S., Giuliani, A., et al. 2007, ATel, 1308, 166

Chugunov, A. I. 2012, Astronomy Letters, 38, 2557

Coleiro, A., Chaty, S., Zurita Heras, J. A., Rahoui, F., \& Tomsick, J. A. 2013, arXiv:1310.0451 66

Combi, J. A., Albacete Colombo, J. F., López-Santiago, J., et al. 2010, A\&A, 522, A50 95, 150

Comella, J. M., Craft, H. D., Lovelace, R. V. R., Sutton, J. M., \& Tyler, G. L. 1969, Nature, 221, 4534

Cooney, A., Dedeo, S., \& Psaltis, D. 2009, PRD, 79(4), 044033120

Cooney, A., Dedeo, S., \& Psaltis, D. 2010, PRD, 82(6), 064033 118, 120, 128

Costantini, E., Pinto, C., Kaastra, J. S., et al. 2012, A\&A, 539, A32 104

Cottam, J., Paerels, F., \& Mendez, M. 2002, Nature, 420, 5126

Courant, R., Friedrichs, K., \& Lewy, H. 1928, Mathematische Annalen, 100, 3259

Cumming, A., 2003, ApJ, 595, 1077 100, 111, 151

Cutler, C. 2002, PRD, 66, 084025 27, 73, 75

D’Aì, A., La Parola, V., Cusumano, G., et al. 2011, A\&A, 529, A30 66

DeDeo, S., \& Psaltis, D. 2003, PRL, 90(14), 141101 118, 132

de Felice, A., \& Tsujikawa, S. 2010, Living Reviews in Relativity, 13, 3 118, 132, 134

Deliduman, C., Ekşi, K. Y., \& Keleş, V. 2012, JCAP, 5, 36118

Demorest, P. B., Pennucci, T., Ransom, S. M., Roberts, M. S. E., \& Hessels, J. W. T. 2010, Nature, 467, $108122,118,137$

den Herder, J. W., Brinkman, A. C., Kahn, S. M., et al. 2001, A\&A, 365, L7 40

Douchin, F., \& Haensel, P. 2001, A\&A, 380, 151 22, 23, 53, 122, 135

Dubner, G. M., Giacani, E. B., Goss, W. M., \& Winkler, P. F. 1994, AJ, 108, 20784

Ducci, L., Sidoli, L., Mereghetti, S., Paizis, A., \& Romano, P. 2009, MNRAS, 398, 2152 52, 64

Ducci, L., Romano, P., \& Esposito, P., et al. 2013, A\&A, 556, A72 66

Duncan, R. C. 2013, Nature, 497, 57472

Duncan, R. C., \& Thompson, C. 1992, ApJL, 392, L9 71

Ekşi, K. Y., Güngör, C., Türkoğlu, M. M. 2014, PRD, 89(6), 063003 (2014). 135

Espinoza, C. M., Lyne, A. G., Stappers, B. W., \& Kramer, M. 2011, MNRAS, 414, 1679 12, 72

Fender, R., Spencer, R., Tzioumis, T., et al. 1998, ApJL, 506, L121 67 
Filipovic, M. D., Payne, J. L., \& Jones, P. A. 2005, Serbian Astronomical Journal, 170, 47 84, 93, 96

Flowers, E. \& Itoh, N. 1976, ApJ, 206, 21832

Flowers, E. \& Rutherman, M. A. 1977, ApJ, 215, 302F 24

Foley, S., Kouveliotou, C., Kaneko, Y., \& Collazzi, A. 2012, GRB Coord. Network, 13280, 172

Franco L. M., Link B., \& Epstein R. I. 2000, ApJ, 543, 987 75, 147

Frieben J., \& Rezzolla L. 2012, MNRAS, 427, 3406 28, 73, 148

Fryxell, B. A., \& Woosley, S. E., 1982, ApJ, 261, 332100

Fujimoto, M. Y., Hanawa, T., \& Miyaji, S. 1981, ApJ, 247, 26717

Gaensler, B. M., Green, A. J., Dubner, G. M., Giacani, E. B., \& Goss, W. M. 1998, Bulletin of the American Astronomical Society, 30, 132884

Galloway, D. K., Muno, M. P., Hartman, J. M., Psaltis, D., \& Chakrabarty, D. 2008, ApJS, 179, 360100 , 102, 110, 151

García, F., Combi, J. A., Albacete-Colombo, J. F., et al. 2012, A\&A, 546, A91 II, 81

García, F., Combi, J. A., Albacete-Colombo, J. F., et al. 2012, BAAA, 55, 479 II, 81

García, F., Zhang, G., \& Méndez, M. 2013, MNRAS, 429, 3266 II, 81

García, F., Aguilera, D. N., \& Romero, G. E. 2014, A\&A, 565, A122 II, 49, 135

García, F., \& Ranea-Sandoval, I. F. 2015, MNRAS, 449, L73 II, 49

Garmire, G. P., Bautz, M. W., Ford, P. G., Nousek, J. A., \& Ricker, G. R., Jr. 2003, Proc. SPIE, 4851, 28 41

Gavriil, F. P., Dib, R., \& Kaspi, V. M. 2011, ApJ, 736, 13872

Geppert, U., \& Urpin, V. 1994, MNRAS, 271, 490 10, 56

Giacconi, R., Gursky, H., Paolini, F. R., \& Rossi, B. R. 1962, PRL, 9, 4394

Glendenning, N. 2000, Compact Stars 2nd. Edition, Springer 12, 21

Gnedin, O. Y., \& Sunyaev, R. A. 1974, A\&A 36, 37910

Gnedin, O. Y., \& Yakovlev, D. G. 1995, Nucl. Phys. A, 582, 69732

Gold, T. 1968, Nature 218, 7314

Grebenev, S. A., \& Sunyaev, R. A. 2007, Astronomy Letters, 33, 149 52, 64

Grindlay, J., Gursky, H., Schnopper, H., Parsignault, D. R., Heise, J., Brinkman, A. C., \& Schrijver, J. 1976, ApJ, 205, L127 100

Gruber, D. E., Blanco, P. R., Heindl, W. A., et al. 1996, A\&AS, 120, 64138

Guillot, S., Rutledge, R. E., \& Brown, E. F. 2011, ApJ, 732, 8826

Gungor, C. \& Yavuz Eksi, K. 2011, ArXiv e-prints/1108.2166 121

Güver, T., Wroblewski, P., Camarota, L., Özel, F. 2010, ApJ, 719, 1807 26, 100, 102, 103, 104, 109, 151

Haensel, P. 2003, en Final Stages of Stellar Evolution, eds. J.M. Hameury y C. Motch, EAS, 200821

Haensel, P., Potekhin, A. Y., \& Yakovlev, D. G. 2007, Neutron Stars 1: Equation of State and Structure, Springer. 10, 11, 22, 26

Haensel, P., \& Pichon, B. 1994, A\&A, 293, 31322

Haensel, P. \& Potekhin, A. Y. 2004, A\&A, 428, 191 121, 122, 135

Haensel, P., \& Zdunik, J. L. 2008, A\&A, 480, 459 23, 24, 53

Halpern, J. P., Gotthelf, E. V., Helfand, D. J., Gezari, S., \& Wegner, G. A. 2004, ATel, 289, 165

Halpern, J. P., \& Gotthelf, E. V. 2004, ATel, 341, 165

Halpern, J. P., \& Gotthelf, E. V. 2010, ApJ, 709, 436 94, 95, 149

Halpern, J. P., Tomsick, J. A., Gotthelf, E. V., et al. 2014, ApJL, 795, L27 150

Hartle, J. B., \& Thorne, K. S. 1968, ApJ, 153, 807153

Hasinger, G., \& van der Klis, M. 1989, A\&A, 225, 79104

Haskell, B., Jones, D. I., \& Andersson, N. 2006, MNRAS, 373, 142326 
Haskell, B., Samuelsson, L., Glampedakis, K., \& Andersson, N. 2008, MNRAS, 385, 53173

Heinke, C. O., Rybicki, G. B., Narayan, R., \& Grindlay, J. E. 2006, ApJ, 644, 109026

Hessels, J. W. T., Ransom, S. M., Stairs, I. H., et al. 2006, Science, 311, 190125

Hewish, A., Bell, S. J., Pilkington, J. D. H., Scott, P. F., \& Collins, R. A. 1968, Nature, 217, 7094

Horowitz, C. J., \& Kadau, K. 2009, PRL, 102, 19110273

Huang, Y. F., \& Geng, J. J. 2014, ApJL, 782, L20 72, 148

Hu Y.-M., Pitkin, M., Heng, I. S., \& Hendry, M. A. 2014, ApJL, 784, L41 72

Hulse, R. A., \& Taylor, J. H. 1974, ApJL 191, 59 5, 13

Hu, W., \& Sawicki, I. 2007, PRD, 76(6), 064004 118, 132

Içdem, B., Baykal, A., \& Inam, S. Ç. 2012, MNRAS, 419, 310972

in't Zand, J. J. M. 2005, A\&A, 441, L1 52, 64

in’t Zand, J. J. M., Homan, J., Keek, L., \& Palmer, D. M. 2012, A\&A, 547, A47 107

in't Zand, J. J. M., \& Weinberg, N. N. 2010, A\&A, 520, A81 107

Ioka, K. 2001, MNRAS, 327, 63973

Itoh, N., \& Kohyama, Y. 1993, ApJ, 404, 268I 32

Jahoda, K., Swank, J. H., Giles, A. B., Stark, M. J., Strohmayer, T., Zhang, W., Morgan, E. H., 1996,

Proc. SPIE, 2808, 59 38, 101

Jaime, L. G., Patiño, L., \& Salgado, M. 2011, PRD, 83(2), 024039 118, 132

Jansen, F., Lumb, D., Altieri, B., et al. 2001, A\&A, 365, L1 39

Jones, P.B. 2004, MNRAS, 351, 95624

Kainulainen, K., Reijonen, V., Sunhede, D. 2007, PRD, 76(4), 043503 132, 134

Kajava, J. J. E., Nättilä, J., Latvala, O.-M., et al. 2014, MNRAS, 445, 4218152

Kantor, E. M., \& Gusakov, M. E. 2014, ApJL, 797, L4 148

Kaspi, V. 2010, PNAS, 107, 7147K 9

Kaspi, V. M., Dieters, S., Strohmayer, T., Jensen, J. B., Roberts, M. S. E., \& Chakrabarty, D. 2003, ApJL, 588, L93 72

Kato, Y. 2007, APSS, 307, 11 15, 66

Kato, Y., Hayashi, M. R., \& Matsumoto, R. 2004, ApJ, 600, 33866

Katz, J. I. 1989, MNRAS, 239, 75173

Katz, J. I. 2013, ApSS, 349, 611148

Keek, L. 2012, ApJ, 756, 130107

Kenter, A. T., Chappell, J. H., Kraft, R. P., et al. 2000, Proc. SPIE, 4012, 46741

Kesteven, M. J. L. 1968, Australian Journal of Physics, 21, 73984

Kesteven, M. J., \& Caswell, J. L. 1987, A\&A, 183, 11884

King, A. R., \& Ritter, H. 1998, MNRAS, 293, L42 15

Komatsu, E., Smith, K. M., Dunkley, J., et al. 2011, ApJS, 192, 18118

Konar, S., \& Bhattacharya, D. 1997, MNRAS, 284, 311 56, 61

Kouveliotou, C., Dieters, S., Strohmayer, T., et al. 1998, Nature, 393, 23572

Kuulkers, E., Homan, J., van der Klis, M., Lewin, W. H. G., \& Méndez, M. 2002, A\&A, 382, 947102

Kuulkers, E., den Hartog, P. R., in’t Zand J. J. M., Verbunt, F. W. M., Harris, W. E., \& Cocchi, M. 2003, A\&A, 399, 663 18, 100

Kuulkers, E., Shaw, S., Paizis, A., et al. 2006, ATel, 874, 166

Kuulkers, E., Shaw, S. E., Paizis, A., et al. 2007, A\&A, 466, 59566

Lai, D., \& Shapiro, S. L. 1991, ApJ, 383, 74527

Lamers, H. J. G. L. M., \& Cassinelli, J. P. 1999, Introduction to Stellar Winds, Cambridge University Press. 98, 150 
Lander, S. K., Andersson, N., Antonopoulou, D., \& Watts, A. L. 2015, MNRAS, 449, 2047148

Larson, D., Dunkley, J., Hinshaw, G., et al. 2011, ApJS, 192, 16118

Lattimer, J. M., \& Prakash, M. 2007, Phys. Rep., 442, 109 7, 25

Levenfish, K. P., \& Yakovlev, D. G. 1994, Astron. Rep., 38, 247

Levine, A. M., Bradt, H., Cui, W., et al. 1996, ApJL, 469, L33 38

Lewin, W. H. G., van Paradijs, J., \& Taam, R. E. 1993, SSRv, 62, 223100

Link, B. 2014, MNRAS, 441, 2676148

Liu, Q.Z., van Paradijs, J., \& van den Heuvel, E. P. J. 2006, A\&A, 455, 1165L 13

Livingstone, M. A., Kaspi, V. M., \& Gavriil, F. P. 2010, ApJ, 710, 171072

London R. A., Taam R. E., \& Howard W. M. 1986, ApJ, 306, 170110

Lorimer, D. R. 2001, Living Reviews in Relativity, 4, 5L 8

Lovelace, R. V. E., Romanova, M. M., \& Bisnovatyi-Kogan, G. S. 2005, ApJ, 625, 95768

Lubiński, P., Bel, M. G., von Kienlin, A., et al. 2005, ATel, 469, 165

Lyutikov, M. 2013, ArXiv e-prints 72

Lyutikov, M. 2015, MNRAS, 447, 1407148

Mana, A., Fatibene, L., \& Ferraris, M. 2015, ArXiv e-prints 132

Manchester, R. N., Hobbs, G. B., Teoh, A., \& Hobbs, M. 2005, AJ, 129, 199313

Mason, K. O., Breeveld, A., Much, R., et al. 2001, A\&A, 365, L36 40

Massi, M., \& Kaufman Bernadó, M. 2008, A\&A, 477, 1 17, 53, 66, 146

Mastrano, A., Suvorov, A. G., \& Melatos, A. 2015, MNRAS, 453, 522148

McClure-Griffiths, N. M., Dickey, J. M., Gaensler, B. M., et al. 2005, ApJS, 158, 17892

Miceli, M., Bocchino, F., Maggio, A., \& Reale, F. 2005, A\&A, 442, 51391

Milne, D. K., Caswell, J. L., Kesteven, M. J., Haynes, R. F., \& Roger, R. S. 1989, Proceedings of the Astronomical Society of Australia, 8, 18784

Miller, M. C. 2013, arXiv:1312.0029 151

Miranda, V., Jorás, S. E., Waga, I., \& Quartin, M. 2009, PRL, $102(22), 221101$ 118, 132

Murata, K., \& Shibazaki, N. 1996, PASJ, 48, 81984

Negueruela, I. 2010, High Energy Phenomena in Massive Stars, 422, 5753

Negueruela, I., Smith, D. M., Reig, P., Chaty, S., \& Torrejón, J. M. 2006, The X-ray Universe 2005, 604, 16552,64

Negueruela, I., Smith, D. M., Torrejón, J. M., \& Reig, P. 2007, ESA Special Publication, 622, 25565

Negueruela, I., Torrejón, J. M., Reig, P., Ribó, M., \& Smith, D. M. 2008, A Population Explosion: The

Nature \& Evolution of X-ray Binaries in Diverse Environments, 1010, 25252,64

Nespoli, E., Fabregat, J., \& Mennickent, R. E. 2008, A\&A, 486, 91165

Nolan, P. L., Abdo, A. A., Ackermann, M., et al. 2012, ApJS, 199, 3165

Olmo, G. J. 2008, PRD, 78, 104026 132, 133

Olmo, G. J. 2011, IJMP D, 20, 413 132, 154

Olmo, G. J., \& Rubiera-Garcia, D. 2013, ArXiv e-prints 135

Oppenheimer, J. R., \& Volkoff, G. M. 1939, Physical Review, 55, 374 3, 20

Orellana, M., García, F., Teppa Pannia, F. A., Romero, G. E. 2013, GR\&G, 45, 771 II, 115

Ostriker, J. P., \& Hartwick, F. D. A. 1968, ApJ, 153, 79773

Owocki, S. P. \& Cohen, D. H. 2006, ApJ, 648, 565 53, 64

Owocki, S. P., Okazaki, A. T., \& Romero, G. E. 2011, IAU Symposium, 272, 587 69, 147

Özel, F., Gould, A., Güver, T. 2012, ApJ, 748, 524

Ozel, F., Psaltis, D., Guver, T., et al. 2015, arXiv:1505.05155 152

Pacini, F. 1967, Nature, 216, 5674 
Pavan, L., Bordas, P., Pühlhofer, G., et al. 2014, A\&A, 562, A122 150

Payne, D. J. B., \& Melatos, A. 2004, MNRAS, 351, 56968

Payne, D. J. B., \& Melatos, A. 2007, MNRAS, 376, 60968

Pearson, K. 1900, Philosophical Magazine Series 5, 50, 302102

Pellizza, L. J., Chaty, S., \& Negueruela, I. 2006, A\&AA, 455, 65352

Percival, W. J., Reid, B. A., Eisenstein, D. J., et al. 2010, MNRAS, 401, 2148118

Pineault, S., Landecker, T. L., \& Routledge, D. 1987, ApJ, 315, 58084

Pons, J. A., Link, B., Miralles, J. A. \& Geppert, U. 2007, PRL, 98, 110110

Pons, J. A., Miralles, J. A., \& Geppert, U. 2009, A\&A, 496, 207 10, 67, 72, 74

Pons, J. A. \& Geppert, U. 2007, A\&A, 470, 303P 34

Potekhin, A. Y. 1999, A\&A, 351, 787 57, 59

Radhakrishnan, V., \& Manchester, R. N. 1969, Nature, 222, 22812

Raedler, K. H. 2000, en Lecture Notes in Physics From the Sun to the Great Attractor, ed. D. Page y J.G.

Hirsch, Berlin Springer Verlag, 556, 10134

Rappaport, S., Ma, C. P., Joss, P. C., \& Nelson, L. A. 1987, ApJ, 322, 842 100, 110

Read, J. S., Lackey, B. D., Owen, B. J., \& Friedman, J.L. 2009, PRD, 79(12), 124032 122, 138

Reijonen, V. 2009, ArXiv e-prints 134, 135

Reisenegger, A. 2003, Proceedings of the International Workshop on Strong Magnetic Fields and Neutron

Stars, 3334

Reisenegger, A. 2013, ArXiv e-prints 27, 73, 75

Reynoso, E. M., Johnston, S., Green, A. J., \& Koribalski, B. S. 2006, MNRAS, 369, 416 84, 92

Rho, J., \& Petre, R. 1998, ApJL, 503, L167 84

Riess, A. G., Macri, L., Casertano, S., et al. 2009, ApJ, 699, 539118

Romano, P., Sidoli, L., Cusumano, G., et al. 2009, ApJ, 696, 20665

Romano, P., Vercellone, S., Krimm, H. A., et al. 2011, arXiv:1111.0698 65

Romero, G. E., Okazaki, A. T, Orellana, M., \& Owocki, S. P. 2007, A\&A, 474, 15 53, 67, 69

Rothschild, R. E., Blanco, P. R., Gruber, D. E., et al. 1998, ApJ, 496, 53838

Runacres, M. C., \& Owocki, S. P. 2005, A\&A, 429, 323 53, 64

Ruderman, M. 1969, Nature, 223, 59712

Sánchez-Ayaso, E., Combi, J. A., Albacete Colombo, J. F., et al. 2012, APSS, 337, 573 95, 150

Santos, E. 2010, PRD, 81(6), 064030121

Santos, E. 2012, Ap\&SS, 341, 411118

Schatz, H., Bildsten, L., Cumming, A., \& Wiescher, M. 1999, ApJ, 524, 1014S 24

Seward, F. D. 1990, ApJS, 73, 78184

Sguera, V., Barlow, E. J., Bird, A. J., et al. 2005, A\&A, 444, 221 52, 64

Sguera, V., Bird, A. J., Dean, A. J., et al. 2006, ATel, 873, 166

Sguera, V., Romero, G. E., Bazzano, A., Masetti, N., Bird, A. J., \& Bassani, L. 2009, ApJ, 697, 1194 52, 65, 66, 69, 146

Sguera, V. 2009, Proceedings of the 7th INTEGRAL Workshop 52, 65, 66

Sguera, V., Ducci, L., Sidoli, L., Bazzano, A., \& Bassani, L. 2010, MNRAS, 402, L49 64

Sguera, V., Drave, S. P., Bird, A. J., et al. 2011, MNRAS, 417, 573 52, 65, 66

Sguera, V. 2013, Nuclear Physics B Proceedings Supplements, 239, 7666

Shapiro, S. \& Teukolsky, S. 1983, Black Holes, White Dwarfs and Neutron Stars, The Physics of Compact Objects, Wiley 7, 11

Shklovsky, I. S. 1968, SvA, 11, 7495

Sidoli, L., Romano, P., Mereghetti, S., et al. 2007, A\&A, 476, 1307 52, 64 
Sidoli, L., Romano, P., Mangano, V., et al. 2008, ApJ, 687, 1230 52, 64

Sidoli, L. 2009, AdSR, 43, 1464 14, 52

Sidoli, L. 2011, AdSR, 48, 8852

Silbar, R. R. \& Reddy, S. 2004, American Journal of Physics, 72, 892 122, 136

Slane, P., Smith, R. K., Hughes, J. P., \& Petre, R. 2002, ApJ, 564, 284 84, 87, 90, 91, 96,97

Smale, A. P., Zhang, W., \& White, N. E. 1997, ApJ, 483, L119 100

Sotiriou, T. P. 2006, C\&QG, 23, 1253 132, 134

Sotiriou, T. P. \& Faraoni, V. 2010, Reviews of Modern Physics, 82, 451 118, 132, 134

Spitkovsky, A., Levin, Y., \& Ushomirsky, G. 2002, ApJ, 566, 1018100

Spruit, H. C. 2010, Lecture Notes in Physics, Berlin Springer Verlag, 794, 233 15, 16

Starobinsky, A. A. 2007, Soviet Journal of Experimental and Theoretical Physics Letters, 86, 157 118, 132

Staykov, K. V., Doneva, D. D., Yazadjiev, S. S., \& Kokkotas, K. D. 2014, JCAP, 10, 006153

Steiner, A. W., Lattimer, J. M., \& Brown, E. F. 2013, ApJL, 765, L5 25, 153

Stella, L., White, N. E., \& Priedhorsky, W. 1987, ApJ, 315, L49 100

Stelle, K. S. 1977, PRD, 16, 953134

Strohmayer, T., \& Bildsten, L. 2006, en Compact Stellar X-Ray Sources, eds Lewin \& van der Klis,

Cambridge University Press 18, 100, 110, 151

Strohmayer, T. E., \& Brown, E. F., 2002, ApJ, 566, 1045 100, 103, 151

Strüder, L., Briel, U., Dennerl, K., et al. 2001, A\&A, 365, L18 40, 85

Suleimanov, V., Poutanen, J., Revnivtsev, M. \& Werner, K. 2011, ApJ, 742, 122 101, 110, 111, 151,152

Swank, J. H., Smith, D. M., \& Markwardt, C. B. 2007, ATel, 999, 165

Tawara Y., Hayakawa S., \& Kii T. 1984, PASJ, 36, 845107

Teppa-Pannia, F. A., García, F., Pérez Bergliafa, S. E., Orellana, M., Romero, G. E. 2016, en preparación. II, 115

Thompson, C., \& Duncan, R. C. 1995, MNRAS, 275, 25575

Thompson, C., \& Duncan, R. C. 1996, ApJ, 473, 32275

Tolman, R. C. 1939, Physical Review, 55, 364 3, 20

Tong H. 2014, ApJ, 784, 8672

Troja, E., Bocchino, F., Miceli, M., \& Reale, F. 2008, A\&A, 485, 777 97, 150

Trümper, J., Pietsch, W., Reppin, C., Voges, W., Staubert, R., \& Kendziorra, E. 1978, ApJL, 219,10510

Turlione, A., Aguilera, D. N., \& Pons, J. A. 2015, A\&A, 577, A5 69, 154

Turner, M. J. L., Abbey, A., Arnaud, M., et al. 2001, A\&A, 365, L27 40, 85

Urpin, V., \& Geppert, U. 1995, MNRAS, 275, 111710

Urpin, V. A., \& Muslimov, A. G. 1992 MNRAS, 256, 26161

Urpin, V. \& Yakovlev, D. G. 1980, SvA, 24, 42432

van Paradijs, J., \& Lewin, H. G. 1986, A\&A, 157, L10 102

Verner, D. A., Ferland, G. J., Korista, K. T., \& Yakovlev, D. G. 1996, ApJ, 465, 487102

Viganò, D., Pons, J. A., \& Miralles, J. A. 2012, Computer Physics Communications, 183, 2042 53, 67,147

Viganò, D., Rea, N., Pons, J. A., Perna, R., Aguilera, D. N., \& Miralles, J. A. 2013, MNRAS, 434, 123 10, $67,74,76,147$

Vink, J. S., de Koter, A., \& Lamers, H. J. G. L. M. 2000, A\&A, 362, 29564

Walter, F. M. \& Matthews, L. D. 1997, Nature, 389, 3589

Walter, R., \& Zurita Heras, J. 2007, A\&A, 476, 335 52, 64

Watts, A. L. 2012, ARA\&A, 50, 609 110, 151

Weisskopf, M. C., Tananbaum, H. D., Van Speybroeck, L. P., \& O’Dell, S. L. 2000, Proc. SPIE, 4012,241

Wen, D.-H., Li, B.-A., \& Chen, L.-W. 2011, ArXiv e-prints/1101.1504 128 
Wentzel, D. G. 1961, ApJ, 133, 17073

White, R. L., \& Long, K. S. 1991, ApJ, 373, 54397

Whiteoak, J. B. Z., \& Green, A. J. 1996, A\&A, 118, 32986

Wijnands, R. 2004, Nucl. Phys. B Proc. Sup., 132, 49614

Wilms, J., Allen, A., \& McCray, R. 2000, ApJ, 542, 914 45, 102

Woods, P. M., Kouveliotou, C., van Paradijs, J., et al. 1999, ApJL, 524, L55 72

Woosley, S. E., \& Weaver, T. A. 1995, ApJS, 101, 181 97, 150

Yagi, K., Kyutoku, K., Pappas, G., Yunes, N., \& Apostolatos, T. A. 2014, PRD, 89, 124013153

Yakovlev, D. G., Kaminker, A. D., Gnedin, O. Y., \& Haensel, P. 2001, Phys. Rep., 354, 1 9, 61

Yazadjiev, S. S., Doneva, D. D., \& Kokkotas, K. D. 2015, PRD, 91, 084018153

Yu, M., Manchester, R. N., Hobbs, G., et al. 2013, MNRAS, 429, 68872

Zavlin, V. E., \& Pavlov, G. G. 1998, A\&A, 329, 58326

Zhang, W., Giles, A. B., Jahoda, K., et al. 1993, Proc. SPIE, 2006, 32438

Zhang W., Smale A. P., Strohmayer T. E., \& Swank J. H., 1998, ApJ, 500, L171 100

Zhang G., Méndez M., Altamirano D., Belloni T. M., \& Homan J., 2009, MNRAS, 398, 368101

Zhang G., Méndez M., \& Altamirano D., 2011, MNRAS, 413, 1913 101, 109, 111, 151

Zhang, G., Méndez, M., Zamfir, M., \& Cumming, A. 2016, MNRAS, 455, 2004151

Zhou, X., Miceli, M., Bocchino, F., Orlando, S., \& Chen, Y. 2011, MNRAS, 415, 244 96, 97

Ziman, J. 1960, Electrons and phonons: The theory of transport phenomena in solids, Clarendon 32 